\title{
Asymmetric Bromoaminocyclization and Desymmetrization of Cyclohexa-1,4-dienes through Anion Phase-Transfer Catalysis
}

Hai-Jiao Long, Yin-Long Li, Bing-Qian Zhang, Wen-Ying Xiao, Xiao-Ying Zhang, Ling He,* and Jun Deng*

Chongqing Key Laboratory of Natural Product Synthesis and Drug Research, School of Pharmaceutical Sciences, Chongqing University, 55 Daxuecheng South Road, Shapingba, Chongqing 401331, China

Email: heling2015@cqu.edu.cn,jdeng@cqu.edu.cn.

\section{Supporting Information}

Table of Contents

General Information S2

Optimization of the Reaction Conditions . $\mathrm{S} 2$

Experimental Procedures for the Substrate Synthesis .$S 5$

General Procedure for the Asymmetric Bromoaminocyclization .$S 14$

Demonstration of Synthetic Utility.

References .$S 27$

Copies of NMR Spectra S29

HPLC Reports. S73

Crystallographic Data of Compounds 2a, $2 \mathrm{~m}$ and 2a' S93 


\section{General Information}

Unless otherwise mentioned, all commercially available reagents were used without further purification. All solvents were purified and dried according to standard methods prior to use. Chromatographic separations were performed using silica gel 200-300 mesh. $1 \mathrm{H}$ and 13C NMR spectra were recorded on $400 \mathrm{MHz}$ (100 MHz for ${ }^{13} \mathrm{C}$ NMR) spectrometers using $\mathrm{CDCl}_{3}$ with TMS or residual solvent as standard unless otherwise noted. Chemical-shift values are given in ppm and referenced to the internal standard, TMS (tetramethylsilane). The peak patterns are indicated as follows: s, singlet; $d$, doublet; t, triplet; q, quadruplet; m, multiplet; $d$, doublet of doublets, and br s, broad singlet. The coupling constants $(J)$ are reported in Hertz $(\mathrm{Hz})$. Melting points were determined using a micromelting point apparatus without corrections. High-resolution mass spectra (HRMS) were recorded on a BioTOFQ mass spectrometer with an ESI source. X-ray crystallography analysis of the single crystal was performed on an Agilent SuperNova-CCD X-ray diffractometer. Optical rotation was obtained from Rudolph Research Analytical Autopol VI automatic polarimeter. Enantiomeric excesses were determined by HPLC using Daicel ${ }^{\circledR}$ Chiralcel OD-H or Daicel ${ }^{\circledR}$ Chiralpak AD-H column using $n$-hexane $/ i-$ $\mathrm{PrOH}$ as a mobile phase and detected by $\mathrm{UV}$ at $254 \mathrm{~nm}$. To ensure the accurate determination of the stereochemical outcome, the obtained product was dissolved in $i-\mathrm{PrOH}$ then the sample was took out from the solution for HPLC analysis. Melting points were measured using a SGWX-4A microscopy melting point meter and are uncorrected.

\section{Optimization of the Reaction Conditions}

\subsection{Preliminary study of the reaction}


Table S1. Preliminary results of the reaction. ${ }^{[a]}$

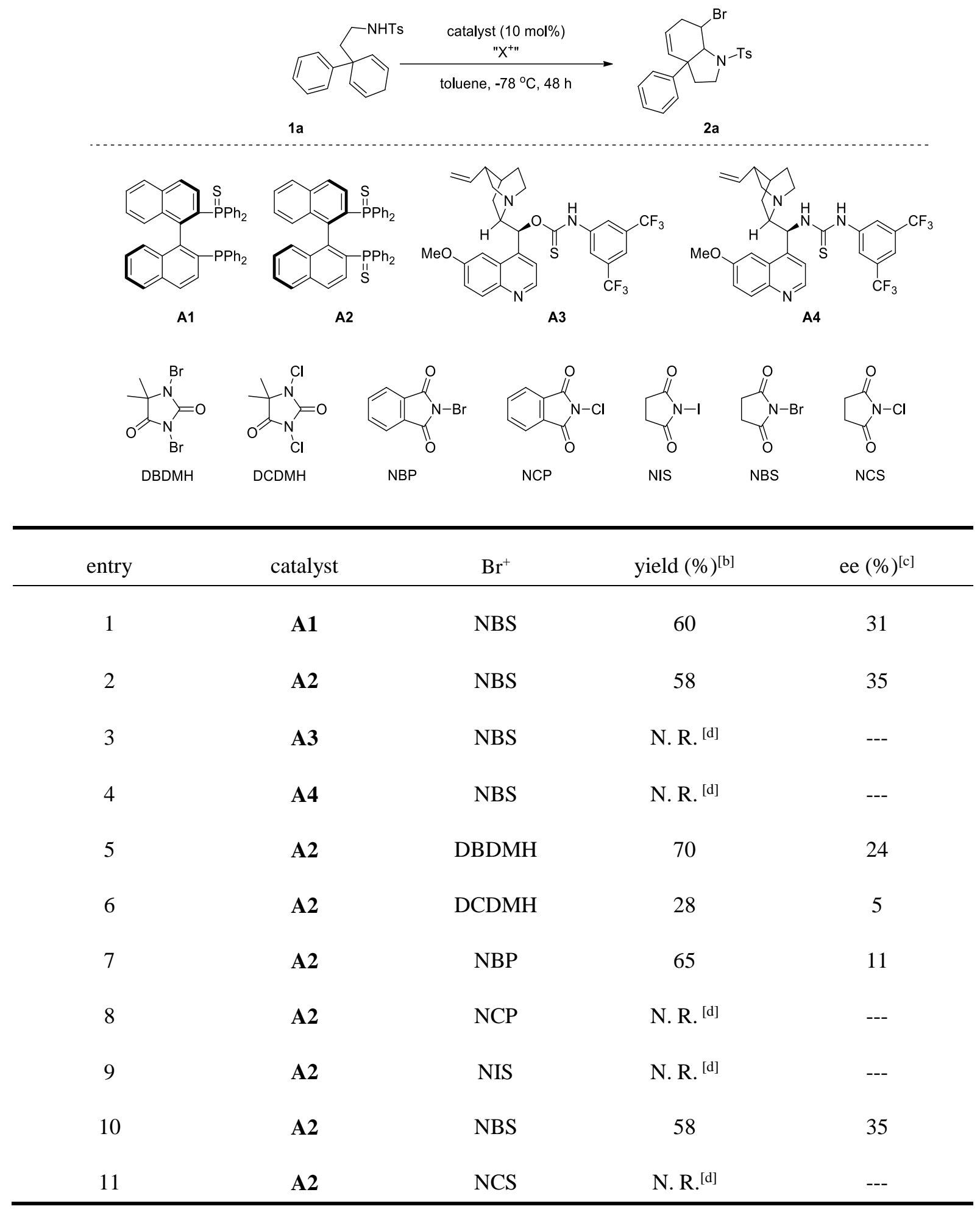

[a] 1a $(0.1 \mathrm{mmol})$, " $\mathrm{X}^{+”}(0.15 \mathrm{mmol})$, catalyst $(0.01 \mathrm{mmol})$, toluene $(2.0 \mathrm{~mL}), \mathrm{T}-78{ }^{\circ} \mathrm{C}, 48 \mathrm{~h}$. [b] Isolated yields. [c] Determined by HPLC on OD-H column. [d] No reaction. 


\subsection{Chiral anion phase transfer catalysis}

Table S2. Screening of the solvents. ${ }^{\text {[a] }}$

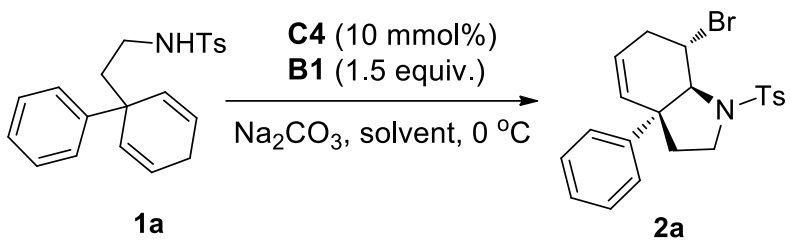

C4:

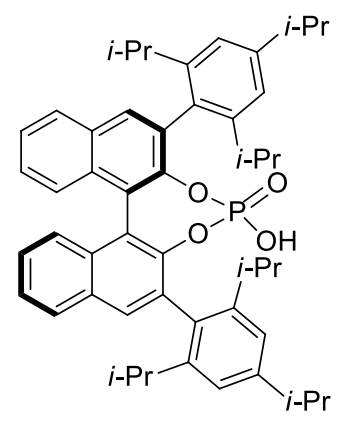

B1:

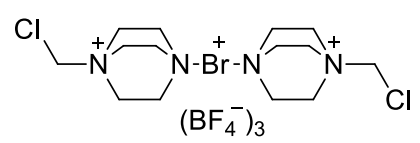

\begin{tabular}{|c|c|c|c|}
\hline entry & solvent & yield $(\%)^{[b]}$ & ee $(\%)^{[\mathrm{c}]}$ \\
\hline 1 & toluene & 75 & 91 \\
\hline 2 & xylene & 65 & 84 \\
\hline 3 & mesitylene & 70 & 89 \\
\hline 4 & benzene & 40 & 82 \\
\hline 5 & trifluorotoluene & 79 & 89 \\
\hline 6 & $\mathrm{CH}_{2} \mathrm{Cl}_{2}$ & 70 & 57 \\
\hline 7 & $\mathrm{ClCH}_{2} \mathrm{CH}_{2} \mathrm{Cl}$ & 77 & 77 \\
\hline 8 & $\mathrm{CHCl}_{3}$ & 74 & 55 \\
\hline 9 & $\mathrm{CCl}_{4}$ & 78 & 97 \\
\hline 10 & Hexane & N. R. ${ }^{[d]}$ & --- \\
\hline 11 & $\mathrm{Et}_{2} \mathrm{O}$ & 56 & 61 \\
\hline 12 & EtOAc & 61 & 53 \\
\hline
\end{tabular}

[a] 1a $(0.1 \mathrm{mmol}), \mathbf{B 1}(0.15 \mathrm{mmol}), \mathbf{C 4}(0.01 \mathrm{mmol}), \mathrm{Na}_{2} \mathrm{CO}_{3}(0.4 \mathrm{mmol})$, solvent $(2.0 \mathrm{~mL}), 0{ }^{\circ} \mathrm{C}, 48 \mathrm{~h}$. [b] Isolated yields. [c] Determined by HPLC on OD-H column. [d] No reaction. 
Table S3. Evaluation of the bases. ${ }^{\text {[a] }}$

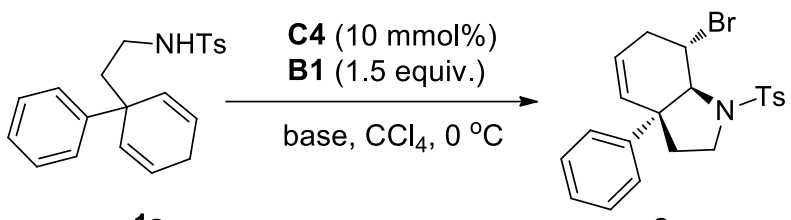

$1 \mathrm{a}$

$2 a$

C4:

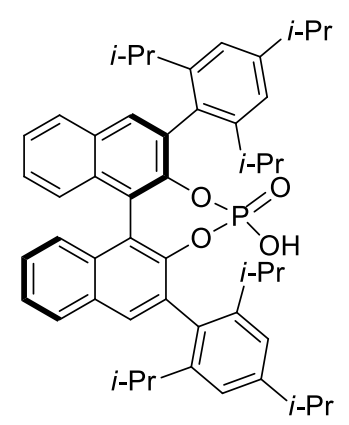

B1:

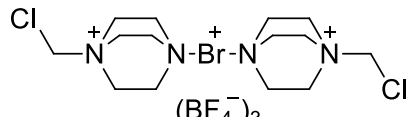
$\left(\mathrm{BF}_{4}^{-}\right)_{3}$

\begin{tabular}{cccc}
\hline entry & base & yield $(\%)^{[\mathrm{b}]}$ & ee $(\%)^{[\mathrm{c}]}$ \\
\hline 1 & $\mathrm{Na}_{2} \mathrm{CO}_{3}$ & 78 & 97 \\
2 & $\mathrm{NaHCO}_{3}$ & 72 & 90 \\
3 & $\mathrm{~K}_{2} \mathrm{CO}_{3}$ & 26 & 82 \\
4 & $\mathrm{~K}_{3} \mathrm{PO}_{4}$ & 15 & 90 \\
5 & $\mathrm{Cs}_{2} \mathrm{CO}_{3}$ & 33 & 92 \\
\hline
\end{tabular}

[a] 1a $(0.1 \mathrm{mmol}), \mathbf{B 1}(0.15 \mathrm{mmol}), \mathbf{C 4}(0.01 \mathrm{mmol})$, base $(0.4 \mathrm{mmol}), \mathrm{CCl}_{4}(2.0 \mathrm{~mL}), 0{ }^{\circ} \mathrm{C}, 48 \mathrm{~h}$. [b] Isolated yields. [c] Determined by HPLC on OD-H column.

\section{Experimental Procedures for the Substrate Synthesis}

Compounds $\mathbf{2} \mathbf{a}-\mathbf{2 h}, \mathbf{2} \mathbf{j}-\mathbf{2 q w e r e}$ prepared according to the procedure of Landais and Zhu. ${ }^{[1]}$

The characterization data of the dienes substrates.

4-Methyl- $N$-[2-(1-phenyl-cyclohexa-2,5-dienyl)-ethyl]benzenesulfonamide $\quad$ (1a): flash

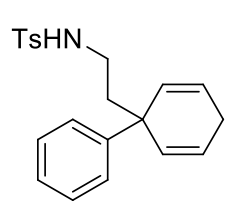
column chromatography (petroleum ether / EtOAc = 10:1), 8 mmol scale, 2.24 g, 79\% yield, white solid, mp: 103-104 ${ }^{\circ} \mathrm{C} .{ }^{1} \mathrm{H}$ NMR (400 MHz, $\left.\mathrm{CDCl}_{3}\right) \delta 7.76$ $(\mathrm{d}, J=7.8 \mathrm{~Hz}, 2 \mathrm{H}), 7.35-7.20(\mathrm{~m}, 6 \mathrm{H}), 7.20-7.12(\mathrm{~m}, 1 \mathrm{H}), 5.83(\mathrm{~d}, J=9.6 \mathrm{~Hz}$, 2H), $5.51(\mathrm{~d}, J=9.9 \mathrm{~Hz}, 2 \mathrm{H}), 4.97(\mathrm{~s}, 1 \mathrm{H}), 3.03(\mathrm{q}, J=7.0 \mathrm{~Hz}, 2 \mathrm{H}), 2.61(\mathrm{q}, J$ $=23.2 \mathrm{~Hz}, 2 \mathrm{H}), 2.43(\mathrm{~s}, 3 \mathrm{H}), 1.98(\mathrm{t}, J=7.5 \mathrm{~Hz}, 2 \mathrm{H}) ;{ }^{13} \mathrm{C} \mathrm{NMR}\left(100 \mathrm{MHz}, \mathrm{CDCl}_{3}\right) \delta$ 131.6, $129.7,128.4,127.1,126.3,126.2,124.5,42.9,40.4,39.0,25.9,21.5$. The spectral data of $1 \mathbf{a}$ 
was consistent with that reported in the literature. ${ }^{1 \mathrm{c}}$

$N$-[2-(1-phenyl-cyclohexa-2,5-dienyl)-ethyl]benzenesulfonamide (1b): flash column

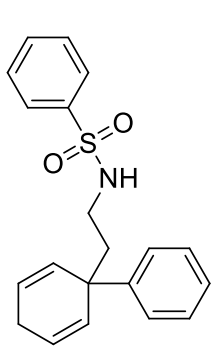
chromatography (petroleum ether / EtOAc = 10:1), 2 mmol scale, $531 \mathrm{mg}, 78 \%$ yield, white solid, mp:157-159 ${ }^{\circ} \mathrm{C} .{ }^{1} \mathrm{H}$ NMR $\left(400 \mathrm{MHz}, \mathrm{CDCl}_{3}\right) \delta 7.89$ (d, $J=$ $7.6 \mathrm{~Hz}, 2 \mathrm{H}), 7.66-7.44(\mathrm{~m}, 3 \mathrm{H}), 7.35-7.22(\mathrm{~m}, 4 \mathrm{H}), 7.19(\mathrm{t}, J=7.2 \mathrm{~Hz}, 1 \mathrm{H})$, 5.92-5.77 (m, 2H), $5.53(\mathrm{~d}, J=9.9 \mathrm{~Hz}, 2 \mathrm{H}), 5.01(\mathrm{t}, J=6.4 \mathrm{~Hz}, 1 \mathrm{H}), 3.06(\mathrm{q}, J$ $=6.9 \mathrm{~Hz}, 2 \mathrm{H}), 2.61(\mathrm{q}, J=23.0 \mathrm{~Hz}, 2 \mathrm{H}), 2.00(\mathrm{t}, J=7.5 \mathrm{~Hz}, 2 \mathrm{H}) ;{ }^{13} \mathrm{C} \mathrm{NMR}$ $\left(100 \mathrm{MHz}, \mathrm{CDCl}_{3}\right) \delta 147.0,140.0,132.6,131.6,129.1,128.4,127.0,126.3$, 126.3, 124.5, 42.9, 40.6, 39.0, 25.9; IR (KBr, cm $\left.{ }^{-1}\right) 2367,1598,1493,1416,1329,1162,1090$, 947, 914, 858, 772, 754; HRMS (M+Na) ${ }^{+}$calcd for $\mathrm{C}_{20} \mathrm{H}_{21} \mathrm{NNaO}_{2} \mathrm{~S} 362.1191$; found 362.1189.

$N$-[2-(1-phenyl-cyclohexa-2,5-dienyl)-ethyl]-4-methoxybenzenesulfonamide (1c): flash

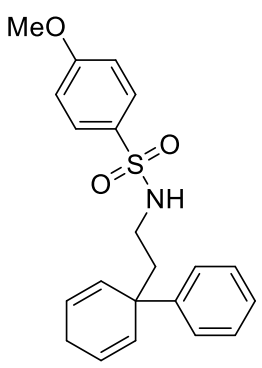

column chromatography (petroleum ether $/$ EtOAc $=10: 1$ ), $2 \mathrm{mmol}$ scale, $582 \mathrm{mg}, 79 \%$ yield, white solid, $\mathrm{mp}=179-181{ }^{\circ} \mathrm{C} .{ }^{1} \mathrm{H}$ NMR $(400 \mathrm{MHz}$, $\left.\mathrm{CDCl}_{3}\right) \delta 7.80(\mathrm{~d}, J=8.8 \mathrm{~Hz}, 2 \mathrm{H}), 7.33-7.20(\mathrm{~m}, 4 \mathrm{H}), 7.18(\mathrm{t}, J=7.0 \mathrm{~Hz}$, $1 \mathrm{H}), 6.98(\mathrm{~d}, J=8.8 \mathrm{~Hz}, 2 \mathrm{H}), 5.84(\mathrm{dt}, J=10.3,3.3 \mathrm{~Hz}, 2 \mathrm{H}), 5.52(\mathrm{~d}, J=$ $10.3 \mathrm{~Hz}, 2 \mathrm{H}), 4.89-4.66(\mathrm{~m}, 1 \mathrm{H}), 3.87(\mathrm{~s}, 3 \mathrm{H}), 3.02(\mathrm{q}, J=6.9 \mathrm{~Hz}, 2 \mathrm{H}), 2.72-$ $2.51(\mathrm{~m}, 2 \mathrm{H}), 1.99(\mathrm{t}, J=7.5 \mathrm{~Hz}, 2 \mathrm{H}) ;{ }^{13} \mathrm{C} \mathrm{NMR}\left(100 \mathrm{MHz}, \mathrm{CDCl}_{3}\right) \delta 162.8$, 147.1, 131.6, 129.2, 128.4, 126.3, 126.2, 124.5, 114.2, 55.7, 42.9, 40.5, 39.0, 25.9; IR (KBr, $\left.\mathrm{cm}^{-1}\right)$ 2866, 1596, 1496, 1464, 1437, 1324, 1154, 1094, 1073, 1024, 945, 881, 829, 740; HRMS $(\mathrm{M}+\mathrm{Na})^{+}$calcd for $\mathrm{C}_{21} \mathrm{H}_{23} \mathrm{NNaO}_{3} \mathrm{~S} 392.1296$; found 392.1296.

$N$-[2-(1-phenyl-cyclohexa-2,5-dienyl)-ethyl]-4-bromobenzenesulfonamide $\quad$ (1d): flash

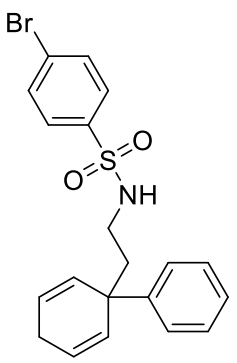
column chromatography (petroleum ether / EtOAc = 10:1), 2 mmol scale, 554 $\mathrm{mg}, 66 \%$ yield, white solid, $\mathrm{mp}=157-158{ }^{\circ} \mathrm{C} .{ }^{1} \mathrm{H} \mathrm{NMR}\left(400 \mathrm{MHz}, \mathrm{CDCl}_{3}\right) \delta$ $7.73(\mathrm{~d}, J=8.2 \mathrm{~Hz}, 2 \mathrm{H}), 7.66(\mathrm{~d}, J=8.7 \mathrm{~Hz}, 2 \mathrm{H}), 7.36-7.13(\mathrm{~m}, 5 \mathrm{H}), 5.86(\mathrm{~d}$, $J=10.1 \mathrm{~Hz}, 2 \mathrm{H}), 5.54(\mathrm{~d}, J=10.3 \mathrm{~Hz}, 2 \mathrm{H}), 5.07-4.88(\mathrm{~m}, 1 \mathrm{H}), 3.04(\mathrm{q}, J=7.0$ $\mathrm{Hz}, 2 \mathrm{H}), 2.63(\mathrm{q}, J=23.2 \mathrm{~Hz}, 2 \mathrm{H}), 2.00(\mathrm{t}, J=7.5 \mathrm{~Hz}, 2 \mathrm{H}) ;{ }^{13} \mathrm{C} \mathrm{NMR}(100$ $\left.\mathrm{MHz}, \mathrm{CDCl}_{3}\right) \delta 146.9,139.1,132.4,131.5,128.6,128.5,127.5,126.3,126.3$, 124.6, 42.9, 40.6, 39.1, 25.9; IR (KBr, $\left.\mathrm{cm}^{-1}\right)$ 2860, 1575, 1490, 1472, 1437, 1331, 1157, 1091, 1068, 1050, 946, 901, 817, 742; HRMS (M-H) ${ }^{-}$calcd for $\mathrm{C}_{20} \mathrm{H}_{19} \mathrm{BrNO}_{2} \mathrm{~S}$ 416.0320; found 416.0306. 
4-Methyl- $N$-[2-(1-(3'-methoxy)-phenyl-cyclohexa-2,5-dienyl)-ethyl]benzenesulfonamide

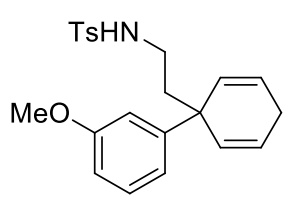

(1f): flash column chromatography (petroleum ether / EtOAc $=10: 1), 4$ mmol scale, $837 \mathrm{mg}, 55 \%$ yield, white solid, $\mathrm{mp}=114-116{ }^{\circ} \mathrm{C} .{ }^{1} \mathrm{H}$ NMR $\left(400 \mathrm{MHz}, \mathrm{CDCl}_{3}\right) \delta 7.75(\mathrm{~d}, J=8.0 \mathrm{~Hz}, 2 \mathrm{H}), 7.30(\mathrm{~d}, J=7.9 \mathrm{~Hz}, 2 \mathrm{H}), 7.20$ $(\mathrm{t}, J=8.0 \mathrm{~Hz}, 1 \mathrm{H}), 6.87-6.77(\mathrm{~m}, 2 \mathrm{H}), 6.76-6.67(\mathrm{~m}, 1 \mathrm{H}), 5.87-5.76(\mathrm{~m}, 2 \mathrm{H})$, $5.51(\mathrm{~d}, J=10.2 \mathrm{~Hz}, 2 \mathrm{H}), 4.99(\mathrm{t}, J=6.2 \mathrm{~Hz}, 1 \mathrm{H}), 3.77(\mathrm{~s}, 3 \mathrm{H}), 3.02(\mathrm{q}, J=6.5 \mathrm{~Hz}, 2 \mathrm{H}), 2.60$ $(\mathrm{q}, J=23.2 \mathrm{~Hz}, 2 \mathrm{H}), 2.43(\mathrm{~s}, 3 \mathrm{H}), 1.95(\mathrm{t}, J=7.5 \mathrm{~Hz}, 2 \mathrm{H}) ;{ }^{13} \mathrm{C} \mathrm{NMR}\left(100 \mathrm{MHz}, \mathrm{CDCl}_{3}\right) \delta$ 159.6, 148.8, 143.3, 137.1, 131.4, 129.7, 129.3, 127.1, 124.5, 118.6, 113.0, 111.0, 55.2, 42.9, $40.5,39.1,25.9,21.5$. The spectral data of $\mathbf{1 f}$ was consistent with that reported in the literature. ${ }^{1 \mathrm{c}}$

4-Methyl- $N$-[2-(1-(3'-benzyloxy)-phenyl-cyclohexa-2,5-dienyl)-ethyl]benzenesulfonamide<smiles></smiles>

(1g): flash column chromatography (petroleum ether / EtOAc = 10:1), 2.4 mmol scale, $747 \mathrm{mg}, 68 \%$ yield, white solid, $\mathrm{mp}=118-120{ }^{\circ} \mathrm{C} .{ }^{1} \mathrm{H}$ NMR $\left(400 \mathrm{MHz}, \mathrm{CDCl}_{3}\right) \delta 7.76(\mathrm{~d}, J=7.9 \mathrm{~Hz}, 2 \mathrm{H}), 7.51-7.36(\mathrm{~m}, 4 \mathrm{H}), 7.36-7.27$ $(\mathrm{m}, 3 \mathrm{H}), 7.22(\mathrm{t}, J=8.0 \mathrm{~Hz}, 1 \mathrm{H}), 6.94-6.74(\mathrm{~m}, 3 \mathrm{H}), 5.89-5.77(\mathrm{~m}, 2 \mathrm{H}), 5.51$ $(\mathrm{d}, J=9.9 \mathrm{~Hz}, 2 \mathrm{H}), 5.02(\mathrm{~s}, 2 \mathrm{H}), 4.88(\mathrm{t}, J=6.2 \mathrm{~Hz}, 1 \mathrm{H}), 3.03(\mathrm{q}, J=6.9 \mathrm{~Hz}, 2 \mathrm{H}), 2.60(\mathrm{q}, J=$ $23.1 \mathrm{~Hz}, 2 \mathrm{H}), 2.42(\mathrm{~s}, 3 \mathrm{H}), 1.96(\mathrm{t}, J=7.5 \mathrm{~Hz}, 2 \mathrm{H}) ;{ }^{13} \mathrm{C} \mathrm{NMR}\left(100 \mathrm{MHz}, \mathrm{CDCl}_{3}\right) \delta 158.9,148.9$, 143.3, 137.1, 137.0, 131.4, 129.7, 129.4, 128.6, 128.0, 127.6, 127.1, 124.6, 118.8, 114.0, 111.8, $70.0,42.9,40.5,39.1,25.9,21.6$. The spectral data of $1 \mathrm{~g}$ was consistent with that reported in the literature. ${ }^{1 \mathrm{c}}$

\section{4-Methyl- $N$-[2-(1-(3'-((tert-butyldimethylsilyl)oxy))-phenyl-cyclohexa-2,5-dienyl)-}

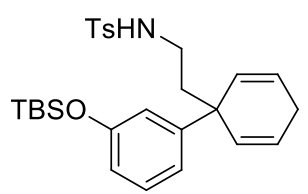

ethyl]benzenesulfonamide (1h): flash column chromatography

(petroleum ether / EtOAc = 10:1), $2.6 \mathrm{mmol}$ scale, $787 \mathrm{mg}, 65 \%$ yield, colorless oil. ${ }^{1} \mathrm{H}$ NMR (400 MHz, $\left.\mathrm{CDCl}_{3}\right) \delta 7.75(\mathrm{~d}, J=7.9 \mathrm{~Hz}, 2 \mathrm{H}), 7.31$ $(\mathrm{d}, J=7.9 \mathrm{~Hz}, 2 \mathrm{H}), 7.13(\mathrm{t}, J=7.9 \mathrm{~Hz}, 1 \mathrm{H}), 6.82(\mathrm{~d}, J=7.8 \mathrm{~Hz}, 1 \mathrm{H}), 6.72$ $(\mathrm{s}, 1 \mathrm{H}), 6.65(\mathrm{~d}, J=7.6 \mathrm{~Hz}, 1 \mathrm{H}), 5.87-5.75(\mathrm{~m}, 2 \mathrm{H}), 5.50(\mathrm{~d}, J=10.0 \mathrm{~Hz}, 2 \mathrm{H}), 4.82(\mathrm{t}, J=6.1$ $\mathrm{Hz}, 1 \mathrm{H}), 3.02(\mathrm{q}, J=6.9 \mathrm{~Hz}, 2 \mathrm{H}), 2.60(\mathrm{q}, J=22.9,22.1 \mathrm{~Hz}, 2 \mathrm{H}), 2.43(\mathrm{~s}, 3 \mathrm{H}), 1.94(\mathrm{t}, J=7.3$ $\mathrm{Hz}, 2 \mathrm{H}), 0.97$ (s, 9H), 0.17 (s, 6H); ${ }^{13} \mathrm{C} \mathrm{NMR}\left(100 \mathrm{MHz}, \mathrm{CDCl}_{3}\right) \delta 155.7,148.7,143.3,137.1$, 131.6, 129.6, 129.1, 127.1, 124.4, 119.0, 118.4, 117.8, 42.8, 40.6, 39.0, 25.8, 25.7, 21.5, 18.2, 4.4. The spectral data of $\mathbf{1 h}$ was consistent with that reported in the literature. ${ }^{\text {1c }}$ 


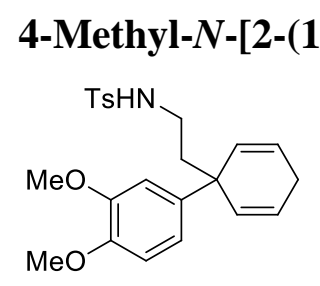

ethyl]benzenesulfonamide (1j): flash column chromatography (petroleum ether $/$ EtOAc $=10: 1), 2 \mathrm{mmol}$ scale, $381 \mathrm{mg}, 46 \%$ yield, white solid, $\mathrm{mp}=$ 142-144 ${ }^{\circ} \mathrm{C} .{ }^{1} \mathrm{H}$ NMR $\left(400 \mathrm{MHz}, \mathrm{CDCl}_{3}\right) \delta 7.73(\mathrm{~d}, J=7.9 \mathrm{~Hz}, 2 \mathrm{H}), 7.29$ $(\mathrm{d}, J=7.9 \mathrm{~Hz}, 2 \mathrm{H}), 6.76(\mathrm{~s}, 3 \mathrm{H}), 5.87-5.76(\mathrm{~m}, 2 \mathrm{H}), 5.49$ (d, $J=10.4 \mathrm{~Hz}$, $2 \mathrm{H}), 4.97(\mathrm{t}, J=6.2 \mathrm{~Hz}, 1 \mathrm{H}), 3.89-3.75(\mathrm{~m}, 6 \mathrm{H}), 3.00(\mathrm{q}, J=6.5 \mathrm{~Hz}, 2 \mathrm{H}), 2.59$ (q, $J=23.1 \mathrm{~Hz}$, 2H), 2.41 (s, 3H), 1.96 (t, $\left.J=7.4 \mathrm{~Hz}, 2 \mathrm{H}) ;{ }^{13} \mathrm{C} \mathrm{NMR} \mathrm{(100} \mathrm{MHz,} \mathrm{CDCl}_{3}\right) \delta 148.7,147.3,143.3$, 139.8, 137.1, 131.8, 129.6, 127.1, 124.3, 118.1, 110.8, 110.1, 55.8, 55.8, 42.5, 40.5, 39.2, 25.8, 21.5. The spectral data of $\mathbf{1} \mathbf{j}$ was consistent with that reported in the literature. ${ }^{1 \mathrm{c}}$

\section{4-Methyl- $N$-[2-(1-(3'-methoxy-4'-((tert-butyldimethylsilyl)oxy))-phenyl-cyclohexa-2,5-}

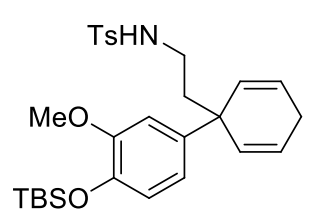

dienyl)-ethyl]benzenesulfonamide (1k): flash column chromatography (petroleum ether / EtOAc = 10:1), 3 mmol scale, $703 \mathrm{mg}, 46 \%$ yield, white solid, $\mathrm{mp}=111-113{ }^{\circ} \mathrm{C} .{ }^{1} \mathrm{H} \mathrm{NMR}\left(400 \mathrm{MHz}, \mathrm{CDCl}_{3}\right) \delta 7.74(\mathrm{~d}, J=8.1 \mathrm{~Hz}$, $2 \mathrm{H}), 7.30(\mathrm{~d}, J=8.3 \mathrm{~Hz}, 2 \mathrm{H}), 6.77-6.69(\mathrm{~m}, 2 \mathrm{H}), 6.65(\mathrm{dd}, J=8.2,2.0 \mathrm{~Hz}$, $1 \mathrm{H}), 5.85-5.76(\mathrm{~m}, 2 \mathrm{H}), 5.50(\mathrm{~d}, J=10.4 \mathrm{~Hz}, 2 \mathrm{H}), 4.90(\mathrm{t}, J=6.2 \mathrm{~Hz}, 1 \mathrm{H}), 3.74(\mathrm{~s}, 3 \mathrm{H}), 3.00$ $(\mathrm{q}, J=6.6 \mathrm{~Hz}, 2 \mathrm{H}), 2.59(\mathrm{q}, J=23.3 \mathrm{~Hz}, 2 \mathrm{H}), 2.42(\mathrm{~s}, 3 \mathrm{H}), 1.94(\mathrm{t}, J=7.5 \mathrm{~Hz}, 2 \mathrm{H}), 0.98(\mathrm{~s}$, 9H), $0.13(\mathrm{~s}, 6 \mathrm{H}) ;{ }^{13} \mathrm{C}$ NMR (100 MHz, $\left.\mathrm{CDCl}_{3}\right) \delta 150.5,143.3,143.3,140.6,137.1,131.9$, $129.6,127.1,124.1,120.4,118.4,110.9,55.6,42.5,40.5,39.3,25.8,25.7,21.5,18.4,-4.6$. The spectral data of $\mathbf{1 k}$ was consistent with that reported in the literature. ${ }^{1 \mathrm{c}}$

\section{4-Methyl- $N$-[2-(1-(4'-benzyloxy-3'-methoxy)-phenyl-cyclohexa-2,5-dienyl)-}

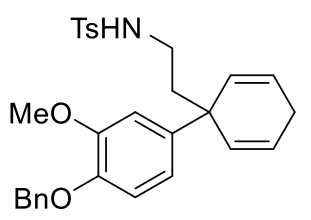

ethyl]benzenesulfonamide (11): flash column chromatography (petroleum ether / EtOAc = 10:1), $1 \mathrm{mmol}$ scale, $316 \mathrm{mg}, 64 \%$ yield, white solid, $\mathrm{mp}$ $=141-144{ }^{\circ} \mathrm{C} 。{ }^{1} \mathrm{H} \mathrm{NMR}\left(400 \mathrm{MHz}, \mathrm{CDCl}_{3}\right) \delta 7.74(\mathrm{~d}, J=8.0 \mathrm{~Hz}, 2 \mathrm{H}), 7.42$ $(\mathrm{d}, J=7.3 \mathrm{~Hz}, 2 \mathrm{H}), 7.35(\mathrm{t}, J=7.4 \mathrm{~Hz}, 2 \mathrm{H}), 7.30(\mathrm{~d}, J=7.7 \mathrm{~Hz}, 3 \mathrm{H}), 6.82-$ $6.76(\mathrm{~m}, 2 \mathrm{H}), 6.70(\mathrm{dd}, J=8.5,2.2 \mathrm{~Hz}, 1 \mathrm{H}), 5.87-5.76(\mathrm{~m}, 2 \mathrm{H}), 5.50(\mathrm{~d}, J=10.2 \mathrm{~Hz}, 2 \mathrm{H}), 5.11$ $(\mathrm{s}, 2 \mathrm{H}), 4.95-4.82(\mathrm{~m}, 1 \mathrm{H}), 3.84(\mathrm{~s}, 3 \mathrm{H}), 3.01(\mathrm{q}, J=6.5 \mathrm{~Hz}, 2 \mathrm{H}), 2.60(\mathrm{q}, J=22.6 \mathrm{~Hz}, 2 \mathrm{H})$, 2.42 (s, 3H), 1.99-1.89 (m, 2H); $\left.{ }^{13} \mathrm{C} \mathrm{NMR} \mathrm{(100} \mathrm{MHz,} \mathrm{CDCl}_{3}\right) \delta 149.3,146.6,143.3,140.4$, 137.2, 137.1, 131.8, 129.6, 128.5, 127.8, 127.2, 127.1, 124.3, 118.2, 113.6, 110.8, 71.0, 56.0, 42.5, 40.5, 39.3, 25.8, 21.5; IR (KBr, $\left.\mathrm{cm}^{-1}\right)$ 2361, 1702, 1596, 1441, 1328, 1291, 1258, 1149 , 1090, 1041, 855, 811, 735, 702; HRMS $(\mathrm{M}+\mathrm{Na})^{+}$calcd for $\mathrm{C}_{29} \mathrm{H}_{31} \mathrm{NNaO}_{4} \mathrm{~S}$ 512.1871; found 512.1878 . 


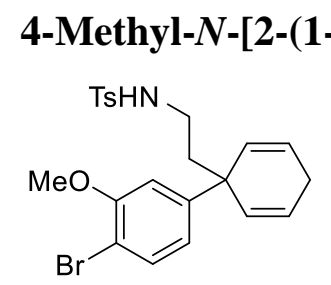

ethyl]benzenesulfonamide (1m): flash column chromatography

(petroleum ether $/$ EtOAc $=10: 1), 1.1 \mathrm{mmol}$ scale, $407 \mathrm{mg}, 80 \%$ yield, colorless oil. ${ }^{1} \mathrm{H}$ NMR $\left(400 \mathrm{MHz}, \mathrm{CDCl}_{3}\right) \delta 7.73(\mathrm{~d}, J=7.9 \mathrm{~Hz}, 2 \mathrm{H}), 7.40$ $(\mathrm{d}, J=8.2 \mathrm{~Hz}, 1 \mathrm{H}), 7.30(\mathrm{~d}, J=7.9 \mathrm{~Hz}, 2 \mathrm{H}), 6.78(\mathrm{~s}, 1 \mathrm{H}), 6.70(\mathrm{~d}, J=8.2$ Hz, 1H), 5.89-5.80 (m, 2H), $5.49(\mathrm{~d}, J=9.9 \mathrm{~Hz}, 2 \mathrm{H}), 4.96(\mathrm{t}, J=6.2 \mathrm{~Hz}, 1 \mathrm{H}), 3.83(\mathrm{~s}, 3 \mathrm{H}), 2.99$ $(\mathrm{q}, J=6.9 \mathrm{~Hz}, 2 \mathrm{H}), 2.69-2.52(\mathrm{~m}, 2 \mathrm{H}), 2.42(\mathrm{~s}, 3 \mathrm{H}), 2.01-1.91(\mathrm{~m}, 2 \mathrm{H}) ;{ }^{13} \mathrm{C} \mathrm{NMR}(100 \mathrm{MHz}$, $\left.\mathrm{CDCl}_{3}\right) \delta 155.6,148.2,143.4,137.0,132.9,131.1,129.7,127.1,124.9,119.7,110.5,109.4$, 56.1, 42.9, 40.4, 39.2, 25.8, 21.6; IR (KBr, cm $\left.{ }^{-1}\right)$ 1651, 1558, 1458, 1395, 1324, 1280, 1259, 1159, 1093, 1049, 1023, 944, 815; HRMS $(\mathrm{M}+\mathrm{Na})^{+}$calcd for $\mathrm{C}_{22} \mathrm{H}_{24} \mathrm{BrNNaO}_{3} \mathrm{~S}$ 484.0558; found 484.0563 .

\section{4-Methyl- $N$-[2-(1-(3'-benzyloxy-4'-bromo)-phenyl-cyclohexa-2,5-dienyl)-}

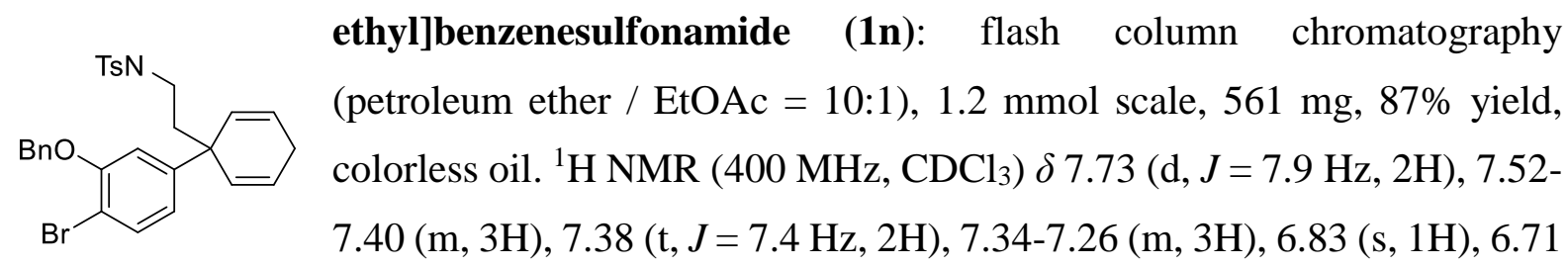
$(\mathrm{d}, J=8.2 \mathrm{~Hz}, 1 \mathrm{H}), 5.90-5.74(\mathrm{~m}, 2 \mathrm{H}), 5.44(\mathrm{~d}, J=10.3 \mathrm{~Hz}, 2 \mathrm{H}), 5.10(\mathrm{~s}, 2 \mathrm{H}), 4.77$ (t, $J=6.1$ $\mathrm{Hz}, 1 \mathrm{H}), 2.98$ (q, $J=7.0 \mathrm{~Hz}, 2 \mathrm{H}), 2.70-2.47(\mathrm{~m}, 2 \mathrm{H}), 2.42$ (s, 3H), 1.98-1.85 (m, 2H); ${ }^{13} \mathrm{C} \mathrm{NMR}$ $\left(100 \mathrm{MHz}, \mathrm{CDCl}_{3}\right) \delta 154.8,147.9,143.4,137.0,136.5,133.0,131.1,129.7,128.5,127.9,127.2$, 127.0, 124.8, 119.9, 112.9, 110.3, 70.8, 42.9, 40.4, 39.1, 25.8, 21.6; IR (KBr, cm $\left.{ }^{-1}\right)$ 1651, 1601, 1557, 1540, 1492, 1457, 1323, 1280, 1160, 1094, 814; HRMS (M-H) ${ }^{-}$calcd for $\mathrm{C}_{28} \mathrm{H}_{27} \mathrm{BrNO}_{3} \mathrm{~S}$ 536.0895 , found 536.0900 .

\section{4-Methyl- $N$-[2-(1-methoxymethyl-cyclohexa-2,5-dienyl)-ethyl]benzenesulfonamide(10):}

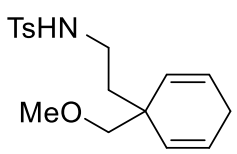

flash column chromatography (petroleum ether / EtOAc $=10: 1), 1.5 \mathrm{mmol}$ scale, $333 \mathrm{mg}, 69 \%$ yield, white solid, $\mathrm{mp}=73-74{ }^{\circ} \mathrm{C} .{ }^{1} \mathrm{H} \mathrm{NMR}(400 \mathrm{MHz}$, $\left.\mathrm{CDCl}_{3}\right) \delta 7.69(\mathrm{~d}, J=8.0 \mathrm{~Hz}, 2 \mathrm{H}), 7.27(\mathrm{~d}, J=8.0 \mathrm{~Hz}, 2 \mathrm{H}), 5.85-5.70(\mathrm{~m}, 2 \mathrm{H})$, $5.36(\mathrm{~d}, J=9.8 \mathrm{~Hz}, 2 \mathrm{H}), 4.83(\mathrm{t}, J=6.1 \mathrm{~Hz}, 1 \mathrm{H}), 3.25(\mathrm{~s}, 3 \mathrm{H}), 3.06(\mathrm{~s}, 2 \mathrm{H}), 2.87(\mathrm{q}, J=6.9 \mathrm{~Hz}$, 2H), 2.68-2.33 (m, 5H), $1.52(\mathrm{t}, J=7.3 \mathrm{~Hz}, 2 \mathrm{H}) ;{ }^{13} \mathrm{C} \mathrm{NMR}\left(100 \mathrm{MHz}, \mathrm{CDCl}_{3}\right) \delta 143.2,136.9$, 129.6, 129.4, 127.0, 126.4, 80.9, 59.4, 40.5, 40.0, 36.7, 26.4, 21.5. The spectral data of 10 was consistent with that reported in the literature. ${ }^{1 \mathrm{c}}$ 
4-Methyl- $N$-[2-(1-benzyloxymethyl-cyclohexa-2,5-dienyl)-ethyl]benzenesulfonamide (1p):

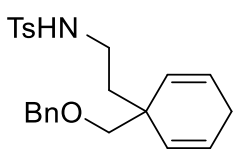

flash column chromatography (petroleum ether / EtOAc $=10: 1$ ), $2.5 \mathrm{mmol}$ scale, $253 \mathrm{mg}, 25 \%$ yield, colorless oil. ${ }^{1} \mathrm{H}$ NMR (400 MHz, $\left.\mathrm{CDCl}_{3}\right) \delta 7.69$ (d, $J=8.0 \mathrm{~Hz}, 2 \mathrm{H}), 7.40-7.21(\mathrm{~m}, 7 \mathrm{H}), 5.85-5.74(\mathrm{~m}, 2 \mathrm{H}), 5.43(\mathrm{~d}, J=10.3 \mathrm{~Hz}$, 2H), $4.75(\mathrm{t}, J=6.1 \mathrm{~Hz}, 1 \mathrm{H}), 4.46(\mathrm{~s}, 2 \mathrm{H}), 3.18(\mathrm{~s}, 2 \mathrm{H}), 2.92(\mathrm{q}, J=6.9 \mathrm{~Hz}, 2 \mathrm{H}), 2.67-2.45(\mathrm{~m}$, 2H), $2.41(\mathrm{~s}, 3 \mathrm{H}), 1.60(\mathrm{t}, J=7.3 \mathrm{~Hz}, 2 \mathrm{H}) ;{ }^{13} \mathrm{C} \mathrm{NMR}\left(100 \mathrm{MHz}, \mathrm{CDCl}_{3}\right) \delta 143.2,138.3,137.0$, $129.6,129.4,128.3$, 127.5, 127.4, 127.0, 126.3, 78.2, 73.3, 40.5, 40.1, 36.8, 26.5, 21.5. The spectral data of $1 \mathbf{p}$ was consistent with that reported in the literature. ${ }^{1 \mathrm{c}}$

\section{4-Methyl- $N$-[2-(1-((tert-butyldimethylsilyl)oxy)methyl-cyclohexa-2,5-dienyl)-ethyl]-}

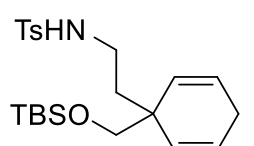

benzenesulfonamide (1q): flash column chromatography (petroleum ether /

EtOAc $=10: 1), 9 \mathrm{mmol}$ scale, $2.39 \mathrm{~g}, 63 \%$ yield, white solid, $\mathrm{mp}=82-84{ }^{\circ} \mathrm{C}$. ${ }^{1} \mathrm{H}$ NMR $\left(400 \mathrm{MHz}, \mathrm{CDCl}_{3}\right) \delta 7.70(\mathrm{~d}, J=8.4 \mathrm{~Hz}, 2 \mathrm{H}), 7.27(\mathrm{~d}, J=8.1 \mathrm{~Hz}$, 2H), 5.79-5.70 (m, 2H), $5.36(\mathrm{~d}, J=10.4 \mathrm{~Hz}, 2 \mathrm{H}), 4.84-4.74(\mathrm{~m}, 1 \mathrm{H}), 3.26(\mathrm{~s}, 2 \mathrm{H}), 2.90(\mathrm{t}, J$ $=7.2 \mathrm{~Hz}, 2 \mathrm{H}), 2.61-2.43(\mathrm{~m}, 2 \mathrm{H}), 2.40(\mathrm{~s}, 3 \mathrm{H}), 1.55(\mathrm{t}, J=7.2 \mathrm{~Hz}, 2 \mathrm{H}), 0.84(\mathrm{~s}, 9 \mathrm{H}),-0.04(\mathrm{~s}$, $6 \mathrm{H}) ;{ }^{13} \mathrm{C} \mathrm{NMR}\left(100 \mathrm{MHz}, \mathrm{CDCl}_{3}\right) \delta 143.1,129.6,129.5,127.0,126.1,71.0,41.2,40.2,36.2$, 26.7, 25.8, 21.5, 18.2, -5.5. The spectral data of 1q was consistent with that reported in the literature. $^{1 \mathrm{c}}$

\subsection{The synthesis of $1 \mathrm{e}, 1 \mathrm{i}^{[1,2]}$}

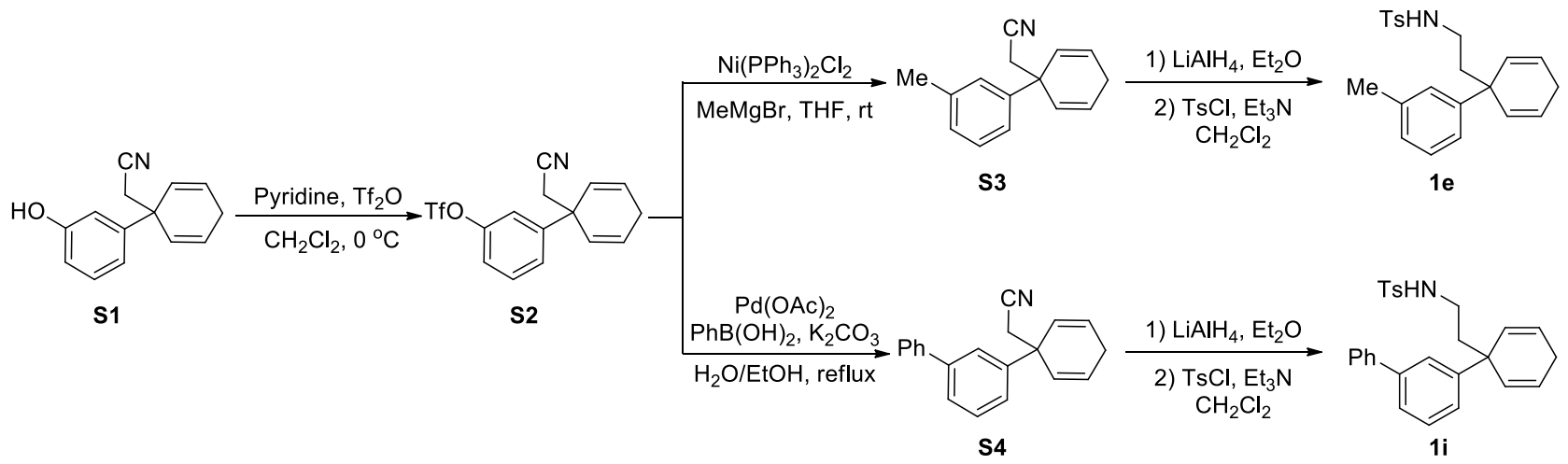

To a solution of the phenol S1 $(2.11 \mathrm{~g}, 10 \mathrm{mmol}, 1.0$ equiv) and pyridine $(1.6 \mathrm{~mL}, 20 \mathrm{mmol}$, 2.0 equiv) in $\mathrm{DCM}(25 \mathrm{~mL})$ was added $\mathrm{Tf}_{2} \mathrm{O}\left(1.8 \mathrm{~mL}, 10.5 \mathrm{mmol}, 1.05\right.$ equiv) at $0{ }^{\circ} \mathrm{C}$. After 
being stirred for 2 hours at $0{ }^{\circ} \mathrm{C}$, the reaction was quenched with water, and the mixture was extracted with $\mathrm{CH}_{2} \mathrm{Cl}_{2}$, washed with brine, the organic layers were dried over $\mathrm{Na}_{2} \mathrm{SO}_{4}$. The filtrate was concentrated to give $\mathbf{S 2}$, which can be used for the next step without purification.

To a solution of $\mathbf{S 2}$ (5 mmol, 1.0 equiv) obtained above in dry THF (12.5 mL) was added $\mathrm{Ni}\left(\mathrm{PPh}_{3}\right) \mathrm{Cl}_{2}$ (327 mg, $0.5 \mathrm{mmol}, 0.1$ equiv) and $\mathrm{MeMgBr}(2.5 \mathrm{~mL}, 7.5 \mathrm{mmol}, 1.5$ equiv) at room temperature. After being stirred overnight, the reaction was quenched with water and extracted with AcOEt. The organic layers were washed with brine, and dried with $\mathrm{Na}_{2} \mathrm{SO}_{4}$. The filtrate was concentrated, the residue was purified by a short column to give $\mathbf{S 3}$, which can be used for the next step without further purification.

To a round-bottom flask was added $\mathbf{S 2}(3.433 \mathrm{~g}, 10 \mathrm{mmol}, 1.0$ equiv), followed by the addition of the following reagents in an $\mathrm{N}_{2}$-filled glovebox. In the following order, phenylboronic acid (1.463 g; $12 \mathrm{mmol}, 1.2$ equiv), triphenylphosphine (105 mg; $0.4 \mathrm{mmol}, 0.04$ equiv); EtOH (33 mL), water $(33 \mathrm{~mL})$, and $\mathrm{Pd}(\mathrm{OAc})_{2}(45 \mathrm{mg} ; 0.2 \mathrm{mmol}, 0.02$ equiv) were added. The reaction mixture was heated at $90{ }^{\circ} \mathrm{C}$ for $12 \mathrm{~h}$. The reaction mixture was cooled to room temperature and EtOH was removed under vacuum. The reaction was extracted with $\mathrm{CH}_{2} \mathrm{Cl}_{2}$ and washed with brine, dried over $\mathrm{Na}_{2} \mathrm{SO}_{4}$. The solvent was removed under vacuum and the residue was purified by flash chromatography $(\mathrm{PE} / \mathrm{EtOAc}=15 / 1)$ to give $\mathbf{S 4}(2.246 \mathrm{~g}$, $83 \%$ yield).

1e and $\mathbf{1 i}$ were prepared from $\mathbf{S 3}$ and $\mathbf{S 4}$ following the procedure of Landais and Zhu. ${ }^{[1]}$

\section{4-Methyl- $N$-[2-(1-(3'-methyl)-phenyl-cyclohexa-2,5-dienyl)-ethyl]benzenesulfonamide}

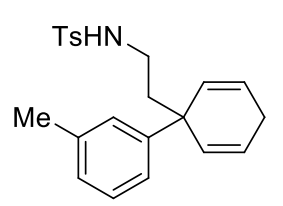

(1e): flash column chromatography (petroleum ether / EtOAc = 10:1), 1.4 mmol scale, $253 \mathrm{mg}, 49 \%$ yield, white solid, $\mathrm{mp}=93-95^{\circ} \mathrm{C} .{ }^{1} \mathrm{H}$ NMR $(400$ $\left.\mathrm{MHz}, \mathrm{CDCl}_{3}\right) \delta 7.76(\mathrm{~d}, J=8.1 \mathrm{~Hz}, 2 \mathrm{H}), 7.32(\mathrm{~d}, J=8.0 \mathrm{~Hz}, 2 \mathrm{H}), 7.18(\mathrm{t}, J$ $=7.9 \mathrm{~Hz}, 1 \mathrm{H}), 7.08-6.95(\mathrm{~m}, 3 \mathrm{H}), 5.88-5.78(\mathrm{~m}, 2 \mathrm{H}), 5.52(\mathrm{~d}, J=10.1 \mathrm{~Hz}$, 2H), $4.85(\mathrm{t}, J=6.2 \mathrm{~Hz}, 1 \mathrm{H}), 3.04$ (q, $J=6.6 \mathrm{~Hz}, 2 \mathrm{H}), 2.62$ (q, $J=23.1 \mathrm{~Hz}, 2 \mathrm{H}), 2.44$ (s, 3H), $2.32(\mathrm{~s}, 3 \mathrm{H}), 1.97(\mathrm{t}, J=7.5 \mathrm{~Hz}, 2 \mathrm{H}),{ }^{13} \mathrm{C} \mathrm{NMR}\left(100 \mathrm{MHz}, \mathrm{CDCl}_{3}\right) \delta 131.7,129.7,128.3,127.1$, 127.0, 124.4, 123.3, 40.6, 39.1, 25.9, 21.6, 21.6. The spectral data of 1e was consistent with that reported in the literature. ${ }^{1 \mathrm{c}}$ 


\section{4-Methyl- $N$-[2-(1-(3'-phenyl)-phenyl-cyclohexa-2,5-dienyl)-ethyl]benzenesulfonamide}

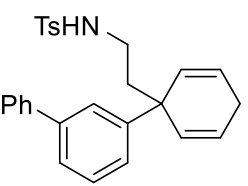

(1i): flash column chromatography (petroleum ether / EtOAc = 10:1), $4 \mathrm{mmol}$ scale, $1.29 \mathrm{~g}, 75 \%$ yield, colorless oil. ${ }^{1} \mathrm{H}$ NMR $\left(400 \mathrm{MHz}, \mathrm{CDCl}_{3}\right) \delta 7.79(\mathrm{~d}$, $J=8.0 \mathrm{~Hz}, 2 \mathrm{H}), 7.57(\mathrm{~d}, J=7.4 \mathrm{~Hz}, 2 \mathrm{H}), 7.50-7.40(\mathrm{~m}, 4 \mathrm{H}), 7.40-7.34(\mathrm{~m}$, $2 \mathrm{H}), 7.30(\mathrm{~d}, J=8.0 \mathrm{~Hz}, 2 \mathrm{H}), 7.27-7.23(\mathrm{~m}, 1 \mathrm{H}), 5.91-5.83(\mathrm{~m}, 2 \mathrm{H}), 5.59(\mathrm{~d}$, $J=10.2 \mathrm{~Hz}, 2 \mathrm{H}), 5.09(\mathrm{t}, J=6.0 \mathrm{~Hz}, 1 \mathrm{H}), 3.13-3.03(\mathrm{~m}, 2 \mathrm{H}), 2.65(\mathrm{q}, J=23.3 \mathrm{~Hz}, 2 \mathrm{H}), 2.40$ (s, 3H), 2.09-2.01 (m, 2H); ${ }^{13} \mathrm{C}$ NMR (100 MHz, $\left.\mathrm{CDCl}_{3}\right) \delta 147.7,143.3,141.4,141.4,137.2$, 131.5, 129.7, 128.8, 128.8, 127.3, 127.1, 125.4, 125.3, 125.2, 124.7, 43.0, 40.6, 39.2, 25.9, 21.5; IR $\left(\mathrm{KBr}, \mathrm{cm}^{-1}\right)$ 1667, 1598, 1477, 1417, 1371, 1326, 1159, 1094, 1021, 949, 888, 814, 760, 738; HRMS (M+Na) ${ }^{+}$calcd for $\mathrm{C}_{27} \mathrm{H}_{27} \mathrm{NNaO}_{2} \mathrm{~S} 452.1660$; found 452.1663 .

\subsection{Synthesis of substrate $1 r^{[1,3]}$}

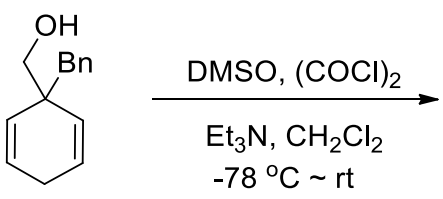

S5

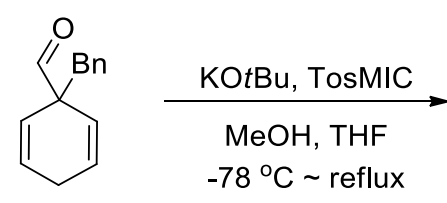

S6

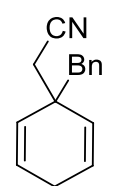

S7

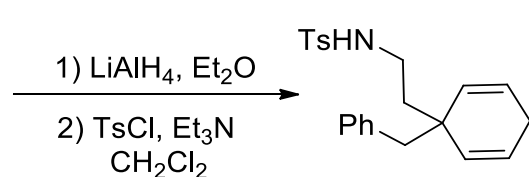

$1 \mathrm{r}$

To a solution of oxalyl chloride $(3.4 \mathrm{~mL}, 40 \mathrm{mmol})$ in $\mathrm{CH}_{2} \mathrm{Cl}_{2}(20 \mathrm{~mL})$ was added DMSO (3.6 mL, $50 \mathrm{mmol}$ ) at $-78{ }^{\circ} \mathrm{C}$. The mixture was stirred for 20 minutes, $\mathbf{S 5}(4.01 \mathrm{~g}, 20 \mathrm{mmol})$ in $\mathrm{CH}_{2} \mathrm{Cl}_{2}(20 \mathrm{~mL})$ was added to the above reaction mixture. After being stirred for 30 minutes, $\mathrm{Et}_{3} \mathrm{~N}(11.1 \mathrm{~mL}, 80 \mathrm{mmol})$ was added to the above reaction mixture, then warmed to room temperature. The reaction was quenched with $\mathrm{NH}_{4} \mathrm{Cl}$, extracted with $\mathrm{CH}_{2} \mathrm{Cl}_{2}$. The organic phase was washed with water and brine, dried over $\mathrm{Na}_{2} \mathrm{SO}_{4}$. The solvent was removed under vacuum and the residue was purified by flash chromatography $(\mathrm{PE} / \mathrm{EtOAc}=15 / 1)$ to give $\mathbf{S 6}(2.31 \mathrm{~g}$, $83 \%$ yield).

To a suspension of KOtBu (583 mg, $5.2 \mathrm{mmol})$ in THF $(20 \mathrm{~mL})$ was added a solution of tosylmethylisocyanide $(664 \mathrm{mg}, 3.4 \mathrm{mmol})$ in THF $(3.4 \mathrm{~mL})$ at $-50{ }^{\circ} \mathrm{C}$. After 30 minutes a solution of aldehyde $\mathbf{S 6}$ (396 mg, $2 \mathrm{mmol}$ ) in THF (2 mL) was added and the reaction mixture was stirred for 1 hours. Then $\mathrm{MeOH}(3.2 \mathrm{~mL})$ was added and the mixture was warmed to reflux 
within 20 minutes and refluxed for another 6 hours. The reaction was quenched with $\mathrm{H}_{2} \mathrm{O}$, extracted with $\mathrm{CH}_{2} \mathrm{Cl}_{2}$. The organic phase was washed with water and brine, dried over $\mathrm{Na}_{2} \mathrm{SO}_{4}$. The solvent was removed under vacuum and the residue was purified by flash chromatography $(\mathrm{PE} / \mathrm{EtOAc}=10 / 1)$ to give $\mathbf{S 7}(523 \mathrm{mg}, 23 \%$ yield $)$.

1r was prepared from $\mathbf{S 7}$ following the procedure of Landais and Zhu. ${ }^{[1]}$

4-Methyl- $N$-[2-(1-benzyl-cyclohexa-2,5-dienyl)-ethyl]-benzenesulfonamide $\quad(1 \mathrm{r}):$ flash TsHN column chromatography (petroleum ether $/$ EtOAc $=10: 1$ ), 3 mmol scale, 900 $\mathrm{mg}, 82 \%$ yield, white solid, $\mathrm{mp}=91-94{ }^{\circ} \mathrm{C} .{ }^{1} \mathrm{H} \mathrm{NMR}\left(400 \mathrm{MHz}, \mathrm{CDCl}_{3}\right) \delta 7.70$ $(\mathrm{d}, J=8.0 \mathrm{~Hz}, 2 \mathrm{H}), 7.28(\mathrm{~d}, J=8.0 \mathrm{~Hz}, 2 \mathrm{H}), 7.23-7.13(\mathrm{~m}, 3 \mathrm{H}), 7.07-6.97$ (m, 2H), $5.69(\mathrm{~d}, J=10.2 \mathrm{~Hz}, 2 \mathrm{H}), 5.32(\mathrm{~d}, J=10.3 \mathrm{~Hz}, 2 \mathrm{H}), 4.78(\mathrm{t}, J=6.0 \mathrm{~Hz}, 1 \mathrm{H}), 2.89$ (q, $J=$ $7.0 \mathrm{~Hz}, 2 \mathrm{H}), 2.55(\mathrm{~s}, 2 \mathrm{H}), 2.46-2.21(\mathrm{~m}, 5 \mathrm{H}), 1.53(\mathrm{t}, J=7.4 \mathrm{~Hz}, 2 \mathrm{H}) ;{ }^{13} \mathrm{C} \mathrm{NMR}(100 \mathrm{MHz}$, $\left.\mathrm{CDCl}_{3}\right) \delta 143.2,137.2,137.0,131.2,130.7,129.6,127.5,127.0,126.0,125.7,49.2,40.7,40.2$, 39.9, 26.1, 21.6; IR (KBr, cm $\left.{ }^{-1}\right)$ 1599, 1494, 1451, 1424, 1322, 1281, 1164, 1117, 1096, 1073, $1012,949,916,809,774,737,702$; $\mathrm{HRMS}(\mathrm{M}+\mathrm{Na})^{+}$calcd for $\mathrm{C}_{22} \mathrm{H}_{25} \mathrm{NNaO}_{2} \mathrm{~S} 390.1504$; found 390.1503 .

1s was prepared following the procedure of Oestreich. ${ }^{[3 a]}$

2-(1-phenyl-cyclohexa-2,5-dienyl)-aceticacid (1s): flash column chromatography (petroleum<smiles>O=C(O)CC1(c2ccccc2)c2ccccc21</smiles>
ether / EtOAc = 2:1), $5 \mathrm{mmol}$ scale, $706 \mathrm{mg}, 66 \%$ yield, white solid, $\mathrm{mp}=89$ $92{ }^{\circ} \mathrm{C} .{ }^{1} \mathrm{H}$ NMR $\left(400 \mathrm{MHz}, \mathrm{CDCl}_{3}\right) \delta 7.40-7.30(\mathrm{~m}, 4 \mathrm{H}), 7.29-7.20(\mathrm{~m}, 1 \mathrm{H}), 5.96-$ $5.81(\mathrm{~m}, 4 \mathrm{H}), 2.92(\mathrm{~s}, 2 \mathrm{H}), 2.73-2.67(\mathrm{~m}, 2 \mathrm{H}) ;{ }^{13} \mathrm{C} \mathrm{NMR}\left(100 \mathrm{MHz}, \mathrm{CDCl}_{3}\right) \delta$ 177.6, 131.1, 128.5, 126.4, 126.2, 124.2, 45.4, 42.5, 25.9; IR (KBr, cm $\left.{ }^{-1}\right) 3037,2886,2730$, 2656, 1708, 1598, 1491, 1443, 1417, 1333, 1243, 1197, 1062, 939, 885, 766; HRMS (M-H)calcd for $\mathrm{C}_{14} \mathrm{H}_{13} \mathrm{O}_{2}$ 213.0916; found 213.0913. 


\section{General Procedure for the Asymmetric Bromoaminocyclization.}
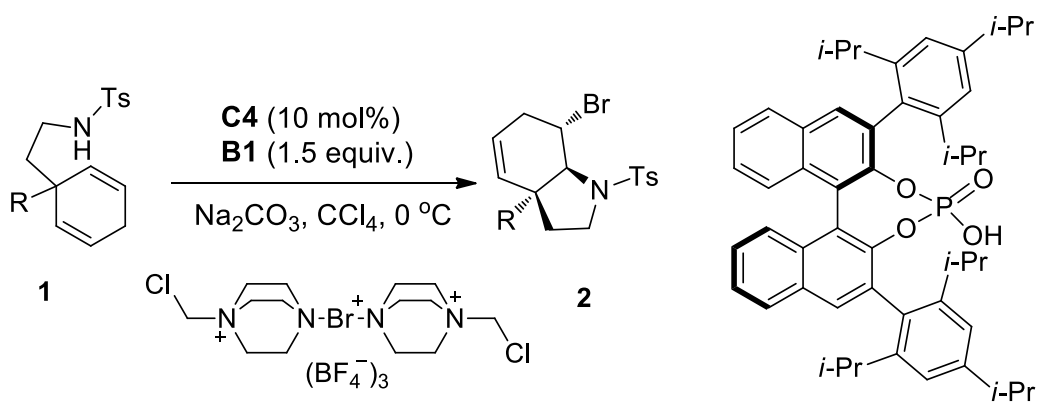

To a flame-dried vial was added $(R)$-TRIP C4 (7.6 mg, $0.010 \mathrm{mmol}, 10 \mathrm{~mol} \%)$, brominating agent $\mathbf{B 1}$ (99 mg, $0.15 \mathrm{mmol}, 1.5$ equiv.), $\mathrm{Na}_{2} \mathrm{CO}_{3}$ (42 mg, $0.4 \mathrm{mmol}, 4.0$ equiv.) and $\mathrm{CCl}_{4}(1.0 \mathrm{~mL})$, the mixture was stirred at $0^{\circ} \mathrm{C}$ for $10 \mathrm{~min}$., then the solution of substrate 1 $(0.1 \mathrm{mmol})$ in $\mathrm{CCl}_{4}(1.0 \mathrm{~mL})$ was added. The reaction was stirred at $0{ }^{\circ} \mathrm{C}$ for $48 \mathrm{~h}$. Upon completion, the reaction was quenched with saturated $\mathrm{Na}_{2} \mathrm{~S}_{2} \mathrm{O}_{3}$ and then was extracted with $\mathrm{CH}_{2} \mathrm{Cl}_{2}$. The combined extracts were washed with brine $(10.0 \mathrm{~mL})$, dried over $\mathrm{Na}_{2} \mathrm{SO}_{4}$ and concentrated in vacuo. The residue was purified by flash column chromatography (petro ether/EtOAc $=15 / 1)$ to yield the product 2.

(3aS,7S,7aS)-7-bromo-3a-phenyl-1-tosyl-2,3,3a,6,7,7a-hexahydro-1H-indole (2a) : flash

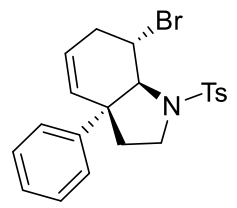

column chromatography (petroleum ether / EtOAc = 15:1), $33.5 \mathrm{mg}, 78 \%$ yield, $97 \%$ ee, white solid, $\mathrm{mp}=171-175^{\circ} \mathrm{C} ;[\alpha]_{\mathrm{D}}{ }^{20}=-198\left(c 0.19, \mathrm{CHCl}_{3}\right) ;{ }^{1} \mathrm{H}$ NMR $\left(400 \mathrm{MHz}, \mathrm{CDCl}_{3}\right) \delta 7.85(\mathrm{~d}, J=8.0 \mathrm{~Hz}, 2 \mathrm{H}), 7.45(\mathrm{~d}, J=7.9 \mathrm{~Hz}, 2 \mathrm{H}), 7.16(\mathrm{~d}$, $J=3.9 \mathrm{~Hz}, 3 \mathrm{H}), 6.95-6.82(\mathrm{~m}, 2 \mathrm{H}), 6.05(\mathrm{dd}, J=9.9,5.0 \mathrm{~Hz}, 1 \mathrm{H}), 5.85(\mathrm{~d}, J=$ $10.3 \mathrm{~Hz}, 1 \mathrm{H}), 4.81(\mathrm{~s}, 1 \mathrm{H}), 4.12(\mathrm{~d}, J=3.2 \mathrm{~Hz}, 1 \mathrm{H}), 3.79$ (dd, $J=12.1,7.4 \mathrm{~Hz}, 1 \mathrm{H}), 3.40(\mathrm{td}, J$ $=12.1,5.0 \mathrm{~Hz}, 1 \mathrm{H}), 3.07(\mathrm{ddt}, J=19.2,4.7,2.4 \mathrm{~Hz}, 1 \mathrm{H}), 2.63-2.47(\mathrm{~m}, 4 \mathrm{H}), 1.74(\mathrm{td}, J=$ $12.3,7.6 \mathrm{~Hz}, 1 \mathrm{H}), 1.61(\mathrm{dd}, J=12.4,4.9 \mathrm{~Hz}, 1 \mathrm{H}) ;{ }^{13} \mathrm{C} \mathrm{NMR}\left(100 \mathrm{MHz}, \mathrm{CDCl}_{3}\right) \delta 144.2,144.0$, 134.6, 130.1, 129.5, 128.1, 127.7, 126.9, 126.5, 125.6, 69.0, 50.4, 48.8, 46.1, 41.5, 28.7, 21.7; IR $\left(\mathrm{KBr}, \mathrm{cm}^{-1}\right)$ 2942, 1347, 1161, 1113, 1090, 1000, 974, 808, 784, 753, 728, 698, 662, 633; HRMS $(\mathrm{M}+\mathrm{Na})^{+}$calcd for $\mathrm{C}_{21} \mathrm{H}_{22} \mathrm{BrNNaO}_{2} \mathrm{~S}$ 454.0452; found 454.0455; HPLC conditions: Daicel Chiralcel OD-H, Hexane $/ i-\mathrm{PrOH}=90: 10,1.0 \mathrm{~mL} / \mathrm{min}, \lambda=254 \mathrm{~nm}, t_{1}=9.8 \mathrm{~min}$ (major), $t_{2}=11.5 \min$ (minor). 
(1S,5R,9S)-9-bromo-5-phenyl-2-tosyl-2-azabicyclo[3.3.1]non-6-ene (2a') : flash column

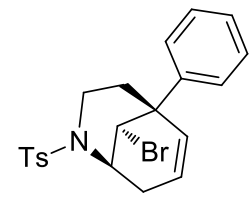
chromatography (petroleum ether / EtOAc $=15: 1$ ), $7.3 \mathrm{mg}, 17 \%$ yield, $97 \%$ ee, white solid, $\mathrm{mp}=147-149{ }^{\circ} \mathrm{C} ;[\alpha]_{\mathrm{D}}{ }^{20}=-138\left(c 0.14, \mathrm{CHCl}_{3}\right)$ 。 ${ }^{1} \mathrm{H}$ NMR $\left(400 \mathrm{MHz}, \mathrm{CDCl}_{3}\right) \delta 7.75(\mathrm{~d}, J=7.9 \mathrm{~Hz}, 2 \mathrm{H}), 7.43-7.22(\mathrm{~m}, 7 \mathrm{H}), 6.05(\mathrm{~d}, J=$ $10.2 \mathrm{~Hz}, 1 \mathrm{H}), 5.88$ (d, $J=10.3 \mathrm{~Hz}, 1 \mathrm{H}), 4.56$ (s, 1H), 4.33 (s, 1H), 3.90 (dd, $J=13.0,4.1 \mathrm{~Hz}, 1 \mathrm{H}), 3.29-3.16(\mathrm{~m}, 1 \mathrm{H}), 2.79-2.66(\mathrm{~m}, 1 \mathrm{H}), 2.47$ (s, 3H), $2.35(\mathrm{td}, J=12.9$, $4.6 \mathrm{~Hz}, 1 \mathrm{H}), 1.92-1.79(\mathrm{~m}, 1 \mathrm{H}), 1.69-1.59(\mathrm{~m}, 1 \mathrm{H}) ;{ }^{13} \mathrm{C} \mathrm{NMR}\left(100 \mathrm{MHz}, \mathrm{CDCl}_{3}\right) \delta 143.6,143.6$, 137.3, 129.9, 128.3, 128.1, 127.1, 127.1, 127.0, 126.2, 56.2, 52.8, 43.5, 39.2, 37.2, 26.3, 21.5. HRMS $(\mathrm{M}+\mathrm{Na})^{+}$calcd for $\mathrm{C}_{21} \mathrm{H}_{22} \mathrm{BrNNaO}_{2} \mathrm{~S}$ 454.0452; found 454.0448; HPLC conditions: Daicel Chiralcel OD-H, Hexane $/ i-\mathrm{PrOH}=90: 10,1.0 \mathrm{~mL} / \mathrm{min}, \lambda=254 \mathrm{~nm}, t_{1}=26.5 \mathrm{~min}$ (minor), $t_{2}=32.5 \min$ (major).

\section{(3aS,7S,7aS)-7-bromo-3a-phenyl-1-(phenylsulfonyl)-2,3,3a,6,7,7a-hexahydro-1H-indole}

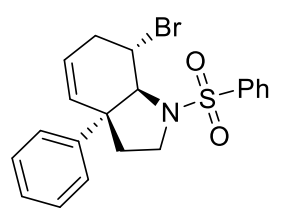

(2b): flash column chromatography (petroleum ether / EtOAc = 15:1), 29.7 $\mathrm{mg}, 71 \%$ yield, $90 \%$ ee, white solid, $\mathrm{mp}=157-159{ }^{\circ} \mathrm{C} ;[\alpha]_{\mathrm{D}}{ }^{20}=-173(c 0.14$, $\left.\mathrm{CHCl}_{3}\right) ;{ }^{1} \mathrm{H} \mathrm{NMR}\left(400 \mathrm{MHz}, \mathrm{CDCl}_{3}\right) \delta 7.97(\mathrm{~d}, J=7.7 \mathrm{~Hz}, 2 \mathrm{H}), 7.76(\mathrm{t}, J=$ $7.5 \mathrm{~Hz}, 1 \mathrm{H}), 7.66(\mathrm{t}, J=7.6 \mathrm{~Hz}, 2 \mathrm{H}), 7.16(\mathrm{~s}, 3 \mathrm{H}), 6.94-6.77(\mathrm{~m}, 2 \mathrm{H}), 6.06$ $(\mathrm{dd}, J=10.7,5.3 \mathrm{~Hz}, 1 \mathrm{H}), 5.85(\mathrm{~d}, J=10.4 \mathrm{~Hz}, 1 \mathrm{H}), 4.82$ (s, 1H), 4.19-4.06 (m, 1H), 3.81 (dd, $J=12.3,7.3 \mathrm{~Hz}, 1 \mathrm{H}), 3.42(\mathrm{td}, J=12.1,5.1 \mathrm{~Hz}, 1 \mathrm{H}), 3.08(\mathrm{~d}, J=19.0 \mathrm{~Hz}, 1 \mathrm{H}), 2.56(\mathrm{dd}, J=$ 19.2, $5.4 \mathrm{~Hz}, 1 \mathrm{H}), 1.71(\mathrm{td}, J=12.3,7.3 \mathrm{~Hz}, 1 \mathrm{H}), 1.64-1.57(\mathrm{~m}, 1 \mathrm{H}) ;{ }^{13} \mathrm{C}$ NMR $(100 \mathrm{MHz}$, $\left.\mathrm{CDCl}_{3}\right) \delta 143.9,137.5,133.3,129.6,129.5,128.1,127.7,126.9,126.6,125.6,69.0,50.4,48.9$, 46.0, 41.4, 28.7; IR (KBr, cm $\left.{ }^{-1}\right) 2953,1473,1351,1165,1114,1090,1022$, 1000, 975, 872, 791, 758, 697, 633; HRMS (M+Na) ${ }^{+}$calcd for $\mathrm{C}_{20} \mathrm{H}_{20} \mathrm{BrNNaO}_{2} \mathrm{~S} 440.0296$; found 440.0286; HPLC conditions: Daicel Chiralcel AD-H, Hexane $/ i-\mathrm{PrOH}=95: 5,1.0 \mathrm{~mL} / \mathrm{min}, \lambda=254 \mathrm{~nm}, t_{1}=11.0$ $\min$ (major), $t_{2}=12.1 \mathrm{~min}$ (minor).

\section{(3aS,7S,7aS)-7-bromo-1-((4-methoxyphenyl)sulfonyl)-3a-phenyl-2,3,3a,6,7,7a-}

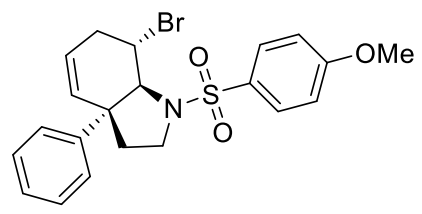

hexahydro-1H-indole (2c): flash column chromatography (petroleum ether / EtOAc = 15:1), $28.5 \mathrm{mg}, 64 \%$ yield, $89 \%$ ee, white solid, $\mathrm{mp}=179-181{ }^{\circ} \mathrm{C} ;[\alpha]_{\mathrm{D}}^{20}=-170\left(c 0.22, \mathrm{CHCl}_{3}\right) ;{ }^{1} \mathrm{H}$ NMR (400 MHz, $\left.\mathrm{CDCl}_{3}\right) \delta 7.89(\mathrm{~d}, J=8.6 \mathrm{~Hz}, 2 \mathrm{H}), 7.24-7.13(\mathrm{~m}$, $3 \mathrm{H}), 7.11(\mathrm{~d}, J=8.6 \mathrm{~Hz}, 2 \mathrm{H}), 6.93(\mathrm{~d}, J=6.3 \mathrm{~Hz}, 2 \mathrm{H}), 6.05(\mathrm{dd}, J=10.8,5.2 \mathrm{~Hz}, 1 \mathrm{H}), 5.85$ (d, $J=10.3 \mathrm{~Hz}, 1 \mathrm{H}), 4.80(\mathrm{~s}, 1 \mathrm{H}), 4.12(\mathrm{~d}, J=3.8 \mathrm{~Hz}, 1 \mathrm{H}), 3.95(\mathrm{~s}, 3 \mathrm{H}), 3.77(\mathrm{dd}, J=12.2,7.4 \mathrm{~Hz}$, 
1H), $3.40(\mathrm{td}, J=12.1,5.1 \mathrm{~Hz}, 1 \mathrm{H}), 3.08(\mathrm{~d}, J=19.1 \mathrm{~Hz}, 1 \mathrm{H}), 2.56(\mathrm{dd}, J=19.0,4.4 \mathrm{~Hz}, 1 \mathrm{H})$, $1.75(\mathrm{td}, J=12.3,7.4 \mathrm{~Hz}, 1 \mathrm{H}), 1.65-1.57(\mathrm{~m}, 1 \mathrm{H}) ;{ }^{13} \mathrm{C} \mathrm{NMR}\left(100 \mathrm{MHz}, \mathrm{CDCl}_{3}\right) \delta 163.4,144.0$, $129.8,129.5,129.2,128.1,126.9,126.5,125.6,114.6,69.0,55.8,50.4,48.8,46.2$, 41.5, 28.7; IR $\left(\mathrm{KBr}, \mathrm{cm}^{-1}\right)$ 2973, 1596, 1497, 1446, 1347, 1259, 1157, 1114, 1092, 1027, 1001, 974, 868, 786, 755, 696, 634; HRMS $(\mathrm{M}+\mathrm{Na})^{+}$calcd for $\mathrm{C}_{21} \mathrm{H}_{22} \mathrm{BrNNaO}_{3} \mathrm{~S} 470.0401$; found 470.0391; HPLC conditions: Daicel Chiralcel OD-H, Hexane $/ i-\mathrm{PrOH}=90: 10,1.0 \mathrm{~mL} / \mathrm{min}, \lambda=254 \mathrm{~nm}$, $t_{1}=16.5 \mathrm{~min}$ (minor), $t_{2}=23.2 \mathrm{~min}$ (major).

\section{(3aS,7S,7aS)-7-bromo-1-((4-bromophenyl)sulfonyl)-3a-phenyl-2,3,3a,6,7,7a-hexahydro-}

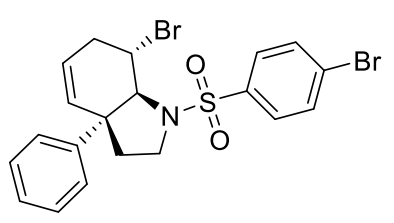

$\mathbf{1 H}$-indole (2d): flash column chromatography (petroleum ether / EtOAc $=15: 1), 38.2 \mathrm{mg}, 77 \%$ yield, $95 \%$ ee, white solid, $\mathrm{mp}=157$ $158{ }^{\circ} \mathrm{C} ;[\alpha]_{\mathrm{D}}{ }^{20}=-106\left(c 0.12, \mathrm{CHCl}_{3}\right) ;{ }^{1} \mathrm{H} \mathrm{NMR}\left(400 \mathrm{MHz}, \mathrm{CDCl}_{3}\right)$ $\delta 7.78(\mathrm{~d}, J=8.8 \mathrm{~Hz}, 4 \mathrm{H}), 7.20(\mathrm{~d}, J=5.6 \mathrm{~Hz}, 3 \mathrm{H}), 6.91(\mathrm{~d}, J=7.3$ $\mathrm{Hz}, 2 \mathrm{H}), 6.08-5.97(\mathrm{~m}, 1 \mathrm{H}), 5.83(\mathrm{~d}, J=10.4 \mathrm{~Hz}, 1 \mathrm{H}), 4.79-4.70(\mathrm{~m}, 1 \mathrm{H}), 4.12(\mathrm{~d}, J=4.3 \mathrm{~Hz}$, $1 \mathrm{H}), 3.73(\mathrm{dd}, J=12.1,7.4 \mathrm{~Hz}, 1 \mathrm{H}), 3.44(\mathrm{td}, J=11.7,5.4 \mathrm{~Hz}, 1 \mathrm{H}), 3.03(\mathrm{~d}, J=18.9 \mathrm{~Hz}, 1 \mathrm{H})$, $2.59(\mathrm{~d}, J=18.7 \mathrm{~Hz}, 1 \mathrm{H}), 1.86(\mathrm{td}, J=12.0,7.5 \mathrm{~Hz}, 1 \mathrm{H}), 1.70(\mathrm{dd}, J=13.0,5.2 \mathrm{~Hz}, 1 \mathrm{H}) ;{ }^{13} \mathrm{C}$ NMR (100 MHz, $\left.\mathrm{CDCl}_{3}\right) \delta 143.6,136.6,132.7,129.8,129.1,128.3,126.8,126.8,125.4,69.5$, 50.7, 48.6, 46.1, 40.9, 29.2; IR (KBr, cm-1) 2941, 1744, 1570, 1471, 1390, 1279, 1217, 1165 , 1066, 1005, 979, 945, 912, 829, 788, 754, 735, 698, 640; HRMS $(\mathrm{M}+\mathrm{Na})^{+}$calcd for $\mathrm{C}_{20} \mathrm{H}_{19} \mathrm{Br}_{2} \mathrm{NNaO}_{2} \mathrm{~S}$ 517.9401; found 517.9389; HPLC conditions: Daicel Chiralcel OD-H, Hexane $/ i-\mathrm{PrOH}=90: 10,1.0 \mathrm{~mL} / \mathrm{min}, \lambda=254 \mathrm{~nm}, t_{1}=12.1 \mathrm{~min}$ (minor), $t_{2}=15.8 \mathrm{~min}$ (major).

(3aS,7S,7aS)-7-bromo-3a-(m-tolyl)-1-tosyl-2,3,3a,6,7,7a-hexahydro-1H-indole (2e): flash

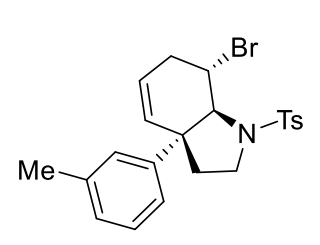

column chromatography (petroleum ether / EtOAc $=15: 1$ ), $30.1 \mathrm{mg}, 67 \%$ yield, 97\% ee, white solid, $\mathrm{mp}=154-156^{\circ} \mathrm{C} ;[\alpha]_{\mathrm{D}}{ }^{20}=-203\left(c 0.09, \mathrm{CHCl}_{3}\right)$; ${ }^{1} \mathrm{H}$ NMR $\left(400 \mathrm{MHz}, \mathrm{CDCl}_{3}\right) \delta 7.85(\mathrm{~d}, J=7.9 \mathrm{~Hz}, 2 \mathrm{H}), 7.45(\mathrm{~d}, J=7.9 \mathrm{~Hz}$, 2H), $7.06(\mathrm{t}, J=7.7 \mathrm{~Hz}, 1 \mathrm{H}), 6.96(\mathrm{~d}, J=7.5 \mathrm{~Hz}, 1 \mathrm{H}), 6.80(\mathrm{~d}, J=7.8 \mathrm{~Hz}$, $1 \mathrm{H}), 6.50(\mathrm{~s}, 1 \mathrm{H}), 6.04(\mathrm{dd}, J=10.7,5.3 \mathrm{~Hz}, 1 \mathrm{H}), 5.84(\mathrm{~d}, J=10.4 \mathrm{~Hz}, 1 \mathrm{H}), 4.80(\mathrm{~s}, 1 \mathrm{H}), 4.08$ (d, $J=3.8 \mathrm{~Hz}, 1 \mathrm{H}), 3.81(\mathrm{dd}, J=12.3,7.3 \mathrm{~Hz}, 1 \mathrm{H}), 3.40(\mathrm{td}, J=12.2,5.0 \mathrm{~Hz}, 1 \mathrm{H}), 3.16-3.00$ (m, 1H), 2.64-2.48 (m, 4H), 2.21 (s, 3H), $1.71(\mathrm{td}, J=12.4,7.4 \mathrm{~Hz}, 1 \mathrm{H}), 1.61-1.55(\mathrm{~m}, 1 \mathrm{H})$; ${ }^{13} \mathrm{C} \mathrm{NMR}\left(100 \mathrm{MHz}, \mathrm{CDCl}_{3}\right) \delta 144.1,143.9,137.5,134.6,130.2,129.6,127.8,127.8,127.3$, 127.2, 125.4, 124.3, 69.0, 50.3, 48.9, 46.2, 41.5, 28.7, 21.7, 21.4; IR (KBr, cm $\left.{ }^{-1}\right)$ 2949, 1734, 1597, 1491, 1448, 1398, 1348, 1254, 1231, 1161, 1090, 1017, 977, 820, 772, 727, 704, 662; 
HRMS $(\mathrm{M}+\mathrm{Na})^{+}$calcd for $\mathrm{C}_{22} \mathrm{H}_{24} \mathrm{BrNNaO}_{2} \mathrm{~S}$ 468.0609; found 468.0599; HPLC conditions: Daicel Chiralcel OD-H, Hexane $/ i-\mathrm{PrOH}=90: 10,1.0 \mathrm{~mL} / \mathrm{min}, \lambda=254 \mathrm{~nm}, t_{1}=9.4 \mathrm{~min}$ (minor), $t_{2}=10.6 \min$ (major).

(3aS,7S,7aS)-7-bromo-3a-(3-methoxyphenyl)-1-tosyl-2,3,3a,6,7,7a-hexahydro-1 $\mathrm{H}$-indole

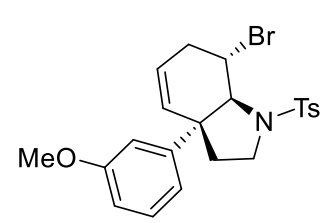

(2f): flash column chromatography (petroleum ether / EtOAc =15:1), 23.8 $\mathrm{mg}, 52 \%$ yield, $85 \%$ ee, white solid, $\mathrm{mp}=140-143{ }^{\circ} \mathrm{C} ;[\alpha]_{\mathrm{D}}{ }^{20}=-181(c$

$\left.0.16, \mathrm{CHCl}_{3}\right) ;{ }^{1} \mathrm{H} \mathrm{NMR}\left(400 \mathrm{MHz}, \mathrm{CDCl}_{3}\right) \delta 7.83(\mathrm{~d}, J=7.9 \mathrm{~Hz}, 2 \mathrm{H}), 7.43$ $(\mathrm{d}, J=7.9 \mathrm{~Hz}, 2 \mathrm{H}), 7.08(\mathrm{t}, J=8.0 \mathrm{~Hz}, 1 \mathrm{H}), 6.73-6.64(\mathrm{~m}, 1 \mathrm{H}), 6.51-6.40$ (m, 2H), $6.04(\mathrm{dd}, J=10.7,5.2 \mathrm{~Hz}, 1 \mathrm{H}), 5.82(\mathrm{~d}, J=10.3 \mathrm{~Hz}, 1 \mathrm{H}), 4.80(\mathrm{~s}, 1 \mathrm{H}), 4.10$ (d, $J=$ $3.9 \mathrm{~Hz}, 1 \mathrm{H}), 3.83-3.67(\mathrm{~m}, 4 \mathrm{H}), 3.40(\mathrm{td}, J=12.1,5.1 \mathrm{~Hz}, 1 \mathrm{H}), 3.07$ (d, $J=19.0 \mathrm{~Hz}, 1 \mathrm{H}), 2.61$ $2.46(\mathrm{~m}, 4 \mathrm{H}), 1.73(\mathrm{td}, J=12.3,7.4 \mathrm{~Hz}, 1 \mathrm{H}), 1.62-1.57(\mathrm{~m}, 1 \mathrm{H}) ;{ }^{13} \mathrm{C} \mathrm{NMR}\left(100 \mathrm{MHz}, \mathrm{CDCl}_{3}\right)$ $\delta 159.3,145.7,144.3,134.5,130.2,129.5,128.9,127.7,125.6,119.3,113.7,111.2,68.9,55.1$, 50.4, 48.8, 46.1, 41.4, 28.8, 21.6; IR (KBr, cm $\left.{ }^{-1}\right)$ 2995, 1604, 1583, 1489, 1431, 1348, 1294 , 1257, 1160, 1091, 1024, 1004, 984, 932, 886, 817, 772, 728, 694, 664; HRMS $(\mathrm{M}+\mathrm{Na})^{+}$calcd for $\mathrm{C}_{22} \mathrm{H}_{24} \mathrm{BrNNaO}_{3} \mathrm{~S}$ 484.0558; found 484.0547; HPLC conditions: Daicel Chiralcel OD-H, Hexane $/ i-\mathrm{PrOH}=95: 5,1.0 \mathrm{~mL} / \mathrm{min}, \lambda=254 \mathrm{~nm}, t_{1}=18.6 \mathrm{~min}$ (minor), $t_{2}=20.8 \mathrm{~min}$ (major).

(3aS,7S,7aS)-3a-(3-(benzyloxy)phenyl)-7-bromo-1-tosyl-2,3,3a,6,7,7a-hexahydro-1H-

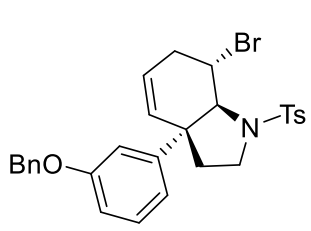

indole (2g): flash column chromatography (petroleum ether $/ \mathrm{EtOAc}=$ $15: 1), 35.0 \mathrm{mg}, 65 \%$ yield, $97 \%$ ee, white solid, $\mathrm{mp}=119-122{ }^{\circ} \mathrm{C} ;[\alpha]_{\mathrm{D}}{ }^{20}$ $=-182\left(c 0.16, \mathrm{CHCl}_{3}\right) ;{ }^{1} \mathrm{H} \mathrm{NMR}\left(400 \mathrm{MHz}, \mathrm{CDCl}_{3}\right) \delta 7.83(\mathrm{~d}, J=7.9 \mathrm{~Hz}$, 2H), 7.52-7.30 (m, 7H), $7.09(\mathrm{t}, J=8.2 \mathrm{~Hz}, 1 \mathrm{H}), 6.77(\mathrm{~d}, J=7.7 \mathrm{~Hz}, 1 \mathrm{H})$, $6.53(\mathrm{~s}, 2 \mathrm{H}), 6.04(\mathrm{dd}, J=10.7,5.2 \mathrm{~Hz}, 1 \mathrm{H}), 5.82$ (d, $J=10.4 \mathrm{~Hz}, 1 \mathrm{H}), 4.95$ (s, 2H), 4.82 (s, $1 \mathrm{H}), 4.13(\mathrm{~d}, J=3.9 \mathrm{~Hz}, 1 \mathrm{H}), 3.78(\mathrm{dd}, J=12.3,7.2 \mathrm{~Hz}, 1 \mathrm{H}), 3.40(\mathrm{td}, J=12.1,5.1 \mathrm{~Hz}, 1 \mathrm{H})$, 3.13-3.02 (m, 1H), $2.56(\mathrm{dd}, J=19.2,5.4 \mathrm{~Hz}, 1 \mathrm{H}), 2.34(\mathrm{~s}, 3 \mathrm{H}), 1.69(\mathrm{td}, J=12.3,7.3 \mathrm{~Hz}, 1 \mathrm{H})$, 1.63-1.56 (m, 1H); ${ }^{13} \mathrm{C}$ NMR (100 MHz, $\left.\mathrm{CDCl}_{3}\right) \delta 158.6,145.7,144.4,136.9,134.4,130.2$, 129.5, 129.0, 128.6, 128.1, 127.9, 127.6, 125.6, 119.6, 114.7, 111.9, 70.0, 69.0, 50.4, 48.8, 46.1, 41.4, 28.7, 21.5; IR (KBr, cm $\left.{ }^{-1}\right)$ 2948, 1581, 1379, 1292, 1240, 1162, 1115, 1091, 1002, 981, 914, 879, 857, 775, 726, 697, 663; HRMS $(\mathrm{M}+\mathrm{Na})^{+}$calcd for $\mathrm{C}_{28} \mathrm{H}_{28} \mathrm{BrNNaO}_{3} \mathrm{~S} 560.0871$; found 560.0860; HPLC conditions: Daicel Chiralcel OD-H, Hexane/i-PrOH $=90: 10,1.0$ $\mathrm{mL} / \mathrm{min}, \lambda=254 \mathrm{~nm}, t_{1}=14.0 \mathrm{~min}$ (minor), $t_{2}=15.5 \mathrm{~min}$ (major). 
(3aS,7S,7aS)-7-bromo-3a-(3-((tert-butyldimethylsilyl)oxy)phenyl)-1-tosyl-2,3,3a,6,7,7a-

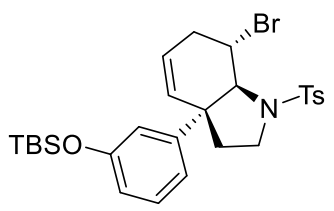

hexahydro-1H-indole (2h): flash column chromatography (petroleum ether $/$ EtOAc = 15:1), $29.5 \mathrm{mg}, 53 \%$ yield, 94\% ee, white solid, $\mathrm{mp}=$ $119-122{ }^{\circ} \mathrm{C} ;[\alpha]_{\mathrm{D}}{ }^{20}=-140\left(c 0.26, \mathrm{CHCl}_{3}\right) ;{ }^{1} \mathrm{H} \mathrm{NMR}\left(400 \mathrm{MHz}, \mathrm{CDCl}_{3}\right)$ $\delta 7.84(\mathrm{~d}, J=8.0 \mathrm{~Hz}, 2 \mathrm{H}), 7.43(\mathrm{~d}, J=7.9 \mathrm{~Hz}, 2 \mathrm{H}), 7.00(\mathrm{t}, J=7.9 \mathrm{~Hz}$, $1 \mathrm{H}), 6.69-6.59(\mathrm{~m}, 2 \mathrm{H}), 6.36(\mathrm{~d}, J=7.2 \mathrm{~Hz}, 1 \mathrm{H}), 6.04(\mathrm{dd}, J=10.8,5.1 \mathrm{~Hz}, 1 \mathrm{H}), 5.80(\mathrm{~d}, J=$ $10.3 \mathrm{~Hz}, 1 \mathrm{H}), 4.84(\mathrm{~s}, 1 \mathrm{H}), 4.13(\mathrm{~d}, J=3.8 \mathrm{~Hz}, 1 \mathrm{H}), 3.72$ (dd, $J=12.0,7.2 \mathrm{~Hz}, 1 \mathrm{H}), 3.39$ (td, $J$ $=12.0,5.1 \mathrm{~Hz}, 1 \mathrm{H}), 3.14-3.04(\mathrm{~m}, 1 \mathrm{H}), 2.60-2.47(\mathrm{~m}, 4 \mathrm{H}), 1.67(\mathrm{td}, J=12.3,7.4 \mathrm{~Hz}, 1 \mathrm{H}), 1.57$ $(\mathrm{dd}, J=12.7,5.2 \mathrm{~Hz}, 1 \mathrm{H}), 0.97(\mathrm{~s}, 9 \mathrm{H}), 0.15(\mathrm{~s}, 6 \mathrm{H}) ;{ }^{13} \mathrm{C} \mathrm{NMR}\left(100 \mathrm{MHz}, \mathrm{CDCl}_{3}\right) \delta 155.3$, 145.6, 144.1, 134.5, 130.1, 129.3, 128.8, 127.7, 125.6, 119.6, 119.5, 118.1, 68.7, 50.1, 48.7, 45.9, 41.3, 28.7, 25.7, 21.7, 18.2, -4.3, -4.4; IR (KBr, cm $\left.{ }^{-1}\right) 2955,1734,1699,1600,1584,1482$, 1355, 1257, 1116, 1092, 993, 961, 940, 910, 872, 781, 745, 696, 664; HRMS $(\mathrm{M}+\mathrm{Na})^{+}$calcd for $\mathrm{C}_{27} \mathrm{H}_{36} \mathrm{BrNNaO}_{3} \mathrm{SSi}$ 584.1266; found 584.1254; HPLC conditions: Daicel Chiralcel OD-H, Hexane $/ i-\mathrm{PrOH}=99.5: 0.5,1.0 \mathrm{~mL} / \mathrm{min}, \lambda=254 \mathrm{~nm}, t_{1}=18.8 \mathrm{~min}$ (major), $t_{2}=22.8 \mathrm{~min}$ (minor).

(3aS,7S,7aS)-3a-([1,1'-biphenyl]-3-yl)-7-bromo-1-tosyl-2,3,3a,6,7,7a-hexahydro-1H-

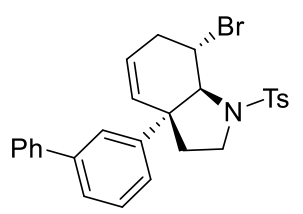

indole (2i): flash column chromatography (petroleum ether $/ \mathrm{EtOAc}=$ $15: 1), 30.3 \mathrm{mg}, 60 \%$ yield, $95 \%$ ee, white solid, $\mathrm{mp}=177-179{ }^{\circ} \mathrm{C} ;[\alpha]_{\mathrm{D}}{ }^{20}=$ -153 (c 0.09, $\left.\mathrm{CHCl}_{3}\right) ;{ }^{1} \mathrm{H} \mathrm{NMR}\left(400 \mathrm{MHz}, \mathrm{CDCl}_{3}\right) \delta 7.84(\mathrm{~d}, J=8.0 \mathrm{~Hz}$, 2H), $7.45(\mathrm{~d}, J=3.7 \mathrm{~Hz}, 4 \mathrm{H}), 7.41-7.32(\mathrm{~m}, 4 \mathrm{H}), 7.26-7.22(\mathrm{~m}, 2 \mathrm{H}), 6.90$ $(\mathrm{d}, J=7.9 \mathrm{~Hz}, 1 \mathrm{H}), 6.09(\mathrm{dd}, J=10.7,5.2 \mathrm{~Hz}, 1 \mathrm{H}), 5.88(\mathrm{~d}, J=10.3 \mathrm{~Hz}, 1 \mathrm{H}), 4.85(\mathrm{~s}, 1 \mathrm{H}), 4.20$ $(\mathrm{d}, J=3.9 \mathrm{~Hz}, 1 \mathrm{H}), 3.77(\mathrm{dd}, J=12.2,7.3 \mathrm{~Hz}, 1 \mathrm{H}), 3.44(\mathrm{td}, J=12.1,5.0 \mathrm{~Hz}, 1 \mathrm{H}), 3.18-3.07$ (m, 1H), 2.59 (dd, $J=19.1,5.5 \mathrm{~Hz}, 1 \mathrm{H}), 2.44$ (s, 3H), 1.77 (td, $J=12.3,7.4 \mathrm{~Hz}, 1 \mathrm{H}), 1.64$ (dd, $J=12.6,4.9 \mathrm{~Hz}, 1 \mathrm{H}) ;{ }^{13} \mathrm{C} \mathrm{NMR}\left(100 \mathrm{MHz}, \mathrm{CDCl}_{3}\right) \delta 144.3,144.1,141.2,141.1,134.4,130.1$, 129.5, 128.6, 128.4, 127.6, 127.3, 126.0, 125.8, 125.6, 68.9, 50.4, 48.7, 46.1, 41.2, 28.8, 21.7; IR $\left(\mathrm{KBr}, \mathrm{cm}^{-1}\right)$ 2937, 1737, 1598, 1477, 1416, 1349, 1245, 1163, 1117, 1023, 999, 970, 944, 895, 801, 787, 755, 701, 664; HRMS $(\mathrm{M}+\mathrm{Na})^{+}$calcd for $\mathrm{C}_{27} \mathrm{H}_{26} \mathrm{BrNNaO}_{2} \mathrm{~S}$ 530.0765; found 530.0757; HPLC conditions: Daicel Chiralcel OD-H, Hexane $/ i-\mathrm{PrOH}=90: 10,1.0 \mathrm{~mL} / \mathrm{min}, \lambda$ $=254 \mathrm{~nm}, t_{1}=10.5 \min$ (major), $t_{2}=12.3 \min$ (minor). 
(3aS,7S,7aS)-7-bromo-3a-(3,4-dimethoxyphenyl)-1-tosyl-2,3,3a,6,7,7a-hexahydro-1H-

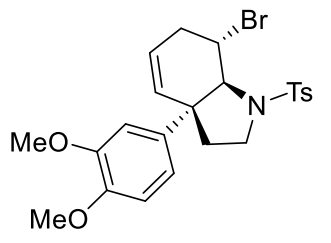

indole (2j): flash column chromatography (petroleum ether $/$ EtOAc $=$ $15: 1), 33.4 \mathrm{mg}, 68 \%$ yield, $86 \%$ ee, white solid, $\mathrm{mp}=168-170{ }^{\circ} \mathrm{C} ;[\alpha]_{\mathrm{D}}{ }^{20}$ $=-167\left(c 0.09, \mathrm{CHCl}_{3}\right) ;{ }^{1} \mathrm{H} \mathrm{NMR}\left(400 \mathrm{MHz}, \mathrm{CDCl}_{3}\right) \delta 7.82(\mathrm{~d}, J=8.2 \mathrm{~Hz}$, $2 \mathrm{H}), 7.40(\mathrm{~d}, J=7.9 \mathrm{~Hz}, 2 \mathrm{H}), 6.65(\mathrm{~d}, J=9.1 \mathrm{~Hz}, 1 \mathrm{H}), 6.51-6.45(\mathrm{~m}, 2 \mathrm{H})$, $6.03(\mathrm{dd}, J=10.1,4.2 \mathrm{~Hz}, 1 \mathrm{H}), 5.79(\mathrm{~d}, J=10.3 \mathrm{~Hz}, 1 \mathrm{H}), 4.80(\mathrm{~s}, 1 \mathrm{H}), 4.06(\mathrm{~d}, J=4.0 \mathrm{~Hz}, 1 \mathrm{H})$, 3.83 (s, 3H), 3.79-3.67 (m, 4H), 3.39 (td, $J=11.9,5.1 \mathrm{~Hz}, 1 \mathrm{H}), 3.14-2.98(\mathrm{~m}, 1 \mathrm{H}), 2.61-2.44$ $(\mathrm{m}, 4 \mathrm{H}), 1.75(\mathrm{td}, J=12.2,7.4 \mathrm{~Hz}, 1 \mathrm{H}), 1.64-1.56(\mathrm{~m}, 1 \mathrm{H}) ;{ }^{13} \mathrm{C} \mathrm{NMR}\left(100 \mathrm{MHz}, \mathrm{CDCl}_{3}\right) \delta$ 148.3, 147.6, 144.0, 136.1, 134.7, 130.0, 129.8, 127.7, 125.6, 119.3, 110.6, 110.5, 68.7, 55.8, 50.2, 48.6, 45.9, 41.0, 28.9, 21.6; IR (KBr, cm $\left.{ }^{-1}\right)$ 2956, 1845, 1699, 1596, 1518, 1462, 1412, 1343, 1262, 1224, 1162, 1113, 1022, 901, 812, 768, 735, 664; HRMS $(\mathrm{M}+\mathrm{Na})^{+}$calcd for $\mathrm{C}_{23} \mathrm{H}_{26} \mathrm{BrNNaO}_{4} \mathrm{~S}$ 514.0664; found 514.0654; HPLC conditions: Daicel Chiralcel OD-H, Hexane $/ i-\mathrm{PrOH}=90: 10,1.0 \mathrm{~mL} / \mathrm{min}, \lambda=254 \mathrm{~nm}, t_{1}=25.8 \mathrm{~min}$ (major), $t_{2}=30.6 \mathrm{~min}$ (minor).

(3aS,7S,7aS)-7-bromo-3a-(4-((tert-butyldimethylsilyl)oxy)-3-methoxyphenyl)-1-tosyl-

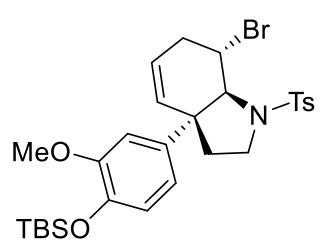

2,3,3a,6,7,7a-hexahydro-1H-indole (2k): flash column chromatography (petroleum ether $/$ EtOAc $=15: 1$ ), $33.6 \mathrm{mg}, 57 \%$ yield, 92\% ee, white solid, $\mathrm{mp}=164-165{ }^{\circ} \mathrm{C} ;[\alpha]_{\mathrm{D}}{ }^{20}=-166\left(c 0.09, \mathrm{CHCl}_{3}\right) ;{ }^{1} \mathrm{H} \mathrm{NMR}(400 \mathrm{MHz}$, $\left.\mathrm{CDCl}_{3}\right) \delta 7.81(\mathrm{~d}, J=7.8 \mathrm{~Hz}, 2 \mathrm{H}), 7.40(\mathrm{~d}, J=7.8 \mathrm{~Hz}, 2 \mathrm{H}), 6.61(\mathrm{~d}, J=$ $8.2 \mathrm{~Hz}, 1 \mathrm{H}), 6.50(\mathrm{~s}, 1 \mathrm{H}), 6.31(\mathrm{~d}, J=8.3 \mathrm{~Hz}, 1 \mathrm{H}), 6.07-5.96(\mathrm{~m}, 1 \mathrm{H}), 5.79(\mathrm{~d}, J=10.4 \mathrm{~Hz}$, $1 \mathrm{H}), 4.79(\mathrm{~s}, 1 \mathrm{H}), 4.05(\mathrm{~s}, 1 \mathrm{H}), 3.82-3.58(\mathrm{~m}, 4 \mathrm{H}), 3.39$ (td, $J=11.9,5.1 \mathrm{~Hz}, 1 \mathrm{H}), 3.15-3.00(\mathrm{~m}$, $1 \mathrm{H}), 2.63-2.42(\mathrm{~m}, 4 \mathrm{H}), 1.78-1.68(\mathrm{~m}, 1 \mathrm{H}), 1.63-1.55(\mathrm{~m}, 1 \mathrm{H}), 0.97(\mathrm{~s}, 9 \mathrm{H}), 0.12(\mathrm{~s}, 6 \mathrm{H}) ;{ }^{13} \mathrm{C}$ $\operatorname{NMR}\left(100 \mathrm{MHz}, \mathrm{CDCl}_{3}\right) \delta 150.2,144.0,143.6,137.1,134.6,130.0,129.9,127.7,125.5,120.2$, 119.3, 111.7, 68.9, 55.5, 50.2, 48.6, 45.9, 40.9, 28.9, 25.7, 21.6, 18.4, -4.6; IR (KBr, cm $\left.{ }^{-1}\right)$ 2945, 1601, 1516, 1467, 1348, 1286, 1161, 1091, 1005, 916, 834, 734, 634; HRMS $(\mathrm{M}+\mathrm{Na})^{+}$calcd for $\mathrm{C}_{28} \mathrm{H}_{38} \mathrm{BrNNaO}_{4} \mathrm{SSi}$ 614.1372; found 614.1390; HPLC conditions: Daicel Chiralcel AD-H, Hexane $/ i-\mathrm{PrOH}=90: 10,1.0 \mathrm{~mL} / \mathrm{min}, \lambda=254 \mathrm{~nm}, t_{1}=5.4 \mathrm{~min}$ (major), $t_{2}=6.1 \mathrm{~min}$ (minor).

(3aS,7S,7aS)-3a-(4-(benzyloxy)-3-methoxyphenyl)-7-bromo-1-tosyl-2,3,3a,6,7,7a-

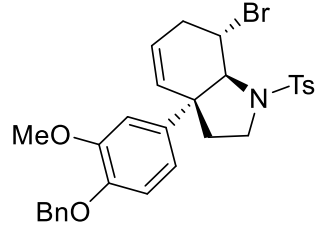

hexahydro-1H-indole (21): flash column chromatography (petroleum ether $/$ EtOAc $=15: 1), 33.6 \mathrm{mg}, 59 \%$ yield, $88 \%$ ee, white solid, $\mathrm{mp}=$ $144-147{ }^{\circ} \mathrm{C} ;[\alpha]_{\mathrm{D}}{ }^{20}=-153\left(c 0.17, \mathrm{CHCl}_{3}\right) ;{ }^{1} \mathrm{H}$ NMR $\left(400 \mathrm{MHz}, \mathrm{CDCl}_{3}\right)$ $\delta 7.82(\mathrm{~d}, J=7.9 \mathrm{~Hz}, 2 \mathrm{H}), 7.48-7.23(\mathrm{~m}, 7 \mathrm{H}), 6.67(\mathrm{~d}, J=8.4 \mathrm{~Hz}, 1 \mathrm{H})$, 
$6.53(\mathrm{~s}, 1 \mathrm{H}), 6.40(\mathrm{~d}, J=8.3 \mathrm{~Hz}, 1 \mathrm{H}), 6.03(\mathrm{dd}, J=10.8,5.0 \mathrm{~Hz}, 1 \mathrm{H}), 5.79(\mathrm{~d}, J=10.4 \mathrm{~Hz}, 1 \mathrm{H})$, $5.10(\mathrm{~s}, 2 \mathrm{H}), 4.80(\mathrm{~s}, 1 \mathrm{H}), 4.06(\mathrm{~d}, J=3.9 \mathrm{~Hz}, 1 \mathrm{H}), 3.81-3.68(\mathrm{~m}, 4 \mathrm{H}), 3.39(\mathrm{td}, J=12.0,5.1$ $\mathrm{Hz}, 1 \mathrm{H}), 3.12-3.02(\mathrm{~m}, 1 \mathrm{H}), 2.62-2.42(\mathrm{~m}, 4 \mathrm{H}), 1.74(\mathrm{td}, J=12.2,7.4 \mathrm{~Hz}, 1 \mathrm{H}), 1.62-1.56(\mathrm{~m}$, $1 \mathrm{H}) ;{ }^{13} \mathrm{C} \mathrm{NMR}\left(100 \mathrm{MHz}, \mathrm{CDCl}_{3}\right) \delta 149.0,146.9,144.0,137.1,136.7,134.7,130.0,129.8$, $128.5,127.8,127.7,127.3,125.6,119.3,113.3,111.3,70.9,68.7,56.0,50.2,48.6,45.9,41.0$, 28.9, 21.6; IR $\left(\mathrm{KBr}, \mathrm{cm}^{-1}\right)$ 2957, 1738, 1593, 1415, 1379, 1331, 1161, 1007, 941, 833, 807, 777, 727, 689, 662, 634; HRMS (M+Na) calcd for $\mathrm{C}_{29} \mathrm{H}_{30} \mathrm{BrNNaO}_{4} \mathrm{~S} 590.0977$; found 590.0990; HPLC conditions: Daicel Chiralcel OD-H, Hexane $/ i$-PrOH $=80: 20,1.0 \mathrm{~mL} / \mathrm{min}, \lambda=254 \mathrm{~nm}$, $t_{1}=26.4 \mathrm{~min}$ (minor), $t_{2}=34.6 \mathrm{~min}$ (major).

(3aS,7S,7aS)-7-bromo-3a-(4-bromo-3-methoxyphenyl)-1-tosyl-2,3,3a,6,7,7a-hexahydro-

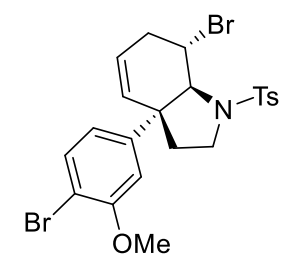

$\mathbf{1 H}$-indole (2m): flash column chromatography (petroleum ether $/ \mathrm{EtOAc}=$ $15: 1), 22.7 \mathrm{mg}, 42 \%$ yield, $88 \%$ ee, white solid, $\mathrm{mp}=170-173{ }^{\circ} \mathrm{C} ;[\alpha]_{\mathrm{D}}{ }^{20}=$ $-127\left(c\right.$ 0.11, $\left.\mathrm{CHCl}_{3}\right) ;{ }^{1} \mathrm{H} \mathrm{NMR}\left(400 \mathrm{MHz}, \mathrm{CDCl}_{3}\right) \delta 7.78(\mathrm{~d}, J=8.1 \mathrm{~Hz}$, $2 \mathrm{H}), 7.38(\mathrm{~d}, J=8.0 \mathrm{~Hz}, 2 \mathrm{H}), 7.24(\mathrm{~d}, J=2.9 \mathrm{~Hz}, 1 \mathrm{H}), 6.67-6.57(\mathrm{~m}, 1 \mathrm{H})$, 6.26-6.16 (m, 1H), $6.03(\mathrm{dd}, J=10.0,4.0 \mathrm{~Hz}, 1 \mathrm{H}), 5.74(\mathrm{~d}, J=10.3 \mathrm{~Hz}, 1 \mathrm{H}), 4.76(\mathrm{~s}, 1 \mathrm{H}), 4.03$ (d, $J=3.7 \mathrm{~Hz}, 1 \mathrm{H}), 3.81-3.66(\mathrm{~m}, 4 \mathrm{H}), 3.38(\mathrm{td}, J=11.8,5.2 \mathrm{~Hz}, 1 \mathrm{H}), 3.10-2.96(\mathrm{~m}, 1 \mathrm{H}), 2.60-$ $2.41(\mathrm{~m}, 4 \mathrm{H}), 1.72(\mathrm{td}, J=12.1,7.5 \mathrm{~Hz}, 1 \mathrm{H}), 1.60(\mathrm{dd}, J=12.6,4.7 \mathrm{~Hz}, 1 \mathrm{H}) ;{ }^{13} \mathrm{C} \mathrm{NMR}(100$ $\left.\mathrm{MHz}, \mathrm{CDCl}_{3}\right) \delta 155.3,144.9,144.2,134.5,132.7,130.1,129.1,127.7,126.2,120.2$, 111.6, 109.8, 68.9, 56.1, 50.5, 48.7, 46.0, 41.0, 28.9, 21.6; IR (KBr, cm $\left.{ }^{-1}\right)$ 2936, 1739, 1593, 1460, 1400, 1351, 1334, 1161, 1007, 937, 816, 776, 728, 665, 632; HRMS $(\mathrm{M}+\mathrm{Na})^{+}$calcd for $\mathrm{C}_{22} \mathrm{H}_{23} \mathrm{Br}_{2} \mathrm{NNaO}_{3} \mathrm{~S}$ 561.9663; found 561.9658; HPLC conditions: Daicel Chiralcel OD-H, Hexane $/ i-\mathrm{PrOH}=90: 10,1.0 \mathrm{~mL} / \mathrm{min}, \lambda=254 \mathrm{~nm}, t_{1}=12.9 \mathrm{~min}$ (major), $t_{2}=15.2 \mathrm{~min}$ (minor).

(3aS,7S,7aS)-3a-(3-(benzyloxy)-4-bromophenyl)-7-bromo-1-tosyl-2,3,3a,6,7,7a-

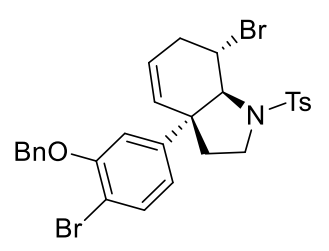

hexahydro-1H-indole (2n): flash column chromatography (petroleum ether $/$ EtOAc = 15:1), $26.6 \mathrm{mg}, 43 \%$ yield, $80 \%$ ee, white solid, $\mathrm{mp}=$ $176-179{ }^{\circ} \mathrm{C} ;[\alpha]_{\mathrm{D}}{ }^{20}=-127\left(c 0.16, \mathrm{CHCl}_{3}\right) ;{ }^{1} \mathrm{H} \mathrm{NMR}\left(400 \mathrm{MHz}, \mathrm{CDCl}_{3}\right)$ $\delta 7.78(\mathrm{~d}, J=8.0 \mathrm{~Hz}, 2 \mathrm{H}), 7.50-7.27(\mathrm{~m}, 8 \mathrm{H}), 6.82-6.75(\mathrm{~m}, 1 \mathrm{H}), 6.25-$ $6.15(\mathrm{~m}, 1 \mathrm{H}), 6.04(\mathrm{dd}, J=9.9,3.9 \mathrm{~Hz}, 1 \mathrm{H}), 5.70(\mathrm{~d}, J=10.7 \mathrm{~Hz}, 1 \mathrm{H}), 5.01(\mathrm{q}, J=11.9 \mathrm{~Hz}$, 2H), 4.80-4.73 (m, 1H), $4.07(\mathrm{~d}, J=4.1 \mathrm{~Hz}, 1 \mathrm{H}), 3.74(\mathrm{dd}, J=12.2,7.2 \mathrm{~Hz}, 1 \mathrm{H}), 3.39(\mathrm{td}, J=$ 11.8, 5.3 Hz, 1H), 3.13-2.96 (m, 1H), 2.64-2.49 (m, 1H), 2.45 (s, 3H), 1.71 (td, $J=12.0,7.4$ $\mathrm{Hz}, 1 \mathrm{H}), 1.65-1.58(\mathrm{~m}, 1 \mathrm{H}) ;{ }^{13} \mathrm{C} \mathrm{NMR}\left(100 \mathrm{MHz}, \mathrm{CDCl}_{3}\right) \delta 154.5,144.8,144.3,136.3,134.5$, 
$132.8,130.0,129.2,128.6,128.1,127.7,127.3,126.2,120.4,114.1,110.7,71.0,68.9,50.4$, 48.5, 46.0, 40.8, 29.0, 21.6; IR (KBr, cm $\left.{ }^{-1}\right)$ 2929, 1651, 1579, 1465, 1394, 1342, 1160, 1019, 947, 813, 733, 703, 667, 642; HRMS $(\mathrm{M}+\mathrm{Na})^{+}$calcd for $\mathrm{C}_{28} \mathrm{H}_{27} \mathrm{Br}_{2} \mathrm{NNaO}_{3} \mathrm{~S}$ 637.9976; found 637.9992; HPLC conditions: Daicel Chiralcel AD-H, Hexane $/ i-\mathrm{PrOH}=90: 10,1.0 \mathrm{~mL} / \mathrm{min}, \lambda$ $=254 \mathrm{~nm}, t_{1}=13.3 \min$ (major), $t_{2}=15.8 \min$ (minor).

\section{(3a $R, 7 S, 7 a S)-7-b r o m o-3 a-(m e t h o x y m e t h y l)-1-t o s y l-2,3,3 a, 6,7,7 a-h e x a h y d r o-1 H$-indole}

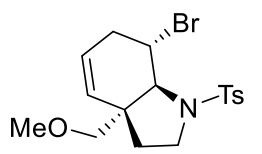

(2o): flash column chromatography (petroleum ether / EtOAc = 15:1), 22.2 $\mathrm{mg}, 56 \%$ yield, $96 \%$ ee, white solid, $\mathrm{mp}=100-103{ }^{\circ} \mathrm{C} ;[\alpha]_{\mathrm{D}}{ }^{20}=-130(c 0.26$, $\left.\mathrm{CHCl}_{3}\right) ;{ }^{1} \mathrm{H} \mathrm{NMR}\left(400 \mathrm{MHz}, \mathrm{CDCl}_{3}\right) \delta 7.75(\mathrm{~d}, J=7.9 \mathrm{~Hz}, 2 \mathrm{H}), 7.32(\mathrm{~d}, J=$ $7.9 \mathrm{~Hz}, 2 \mathrm{H}), 5.86-5.73(\mathrm{~m}, 1 \mathrm{H}), 5.44(\mathrm{~d}, J=9.6 \mathrm{~Hz}, 1 \mathrm{H}), 4.81(\mathrm{~s}, 1 \mathrm{H}), 4.04(\mathrm{~d}, J=3.6 \mathrm{~Hz}, 1 \mathrm{H})$, $3.45(\mathrm{dd}, J=12.1,7.5 \mathrm{~Hz}, 1 \mathrm{H}), 3.39-3.31(\mathrm{~m}, 2 \mathrm{H}), 3.24-3.07(\mathrm{~m}, 4 \mathrm{H}), 2.98-2.85(\mathrm{~m}, 1 \mathrm{H})$, $2.58-2.33(\mathrm{~m}, 4 \mathrm{H}), 1.40(\mathrm{td}, J=12.1,7.5 \mathrm{~Hz}, 1 \mathrm{H}), 1.28(\mathrm{dd}, J=12.2,5.2 \mathrm{~Hz}, 1 \mathrm{H}) ;{ }^{13} \mathrm{C} \mathrm{NMR}$ $\left(100 \mathrm{MHz} \mathrm{CDCl}_{3}\right) \delta 143.7,134.5,129.6,128.5,127.7,125.0,76.3,62.4,59.0,48.1,47.9,47.4$, 34.7, 29.2, 21.6; IR (KBr, cm $\left.{ }^{-1}\right)$ 2939, 1597, 1452, 1383, 1347, 1247, 1159, 1034, 984, 818, 781, 737, 708, 666; HRMS (M+Na) calcd for $\mathrm{C}_{17} \mathrm{H}_{22} \mathrm{BrNNaO}_{3} \mathrm{~S}$ 422.0401; found 422.0407; HPLC conditions: Daicel Chiralcel OD-H, Hexane $/ i-\mathrm{PrOH}=90: 10,1.0 \mathrm{~mL} / \mathrm{min}, \lambda=254 \mathrm{~nm}$, $t_{1}=7.1 \mathrm{~min}$ (minor), $t_{2}=9.0 \mathrm{~min}$ (major).

\section{(3aR,7S,7aS)-3a-((benzyloxy)methyl)-7-bromo-1-tosyl-2,3,3a,6,7,7a-hexahydro-1H-}

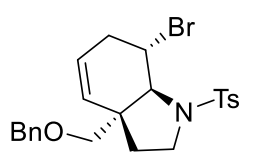

indole (2p): flash column chromatography (petroleum ether $/$ EtOAc $=$ $15: 1), 22.6 \mathrm{mg}, 48 \%$ yield, $94 \%$ ee, white solid, $\mathrm{mp}=127-130{ }^{\circ} \mathrm{C} ;[\alpha]_{\mathrm{D}}{ }^{20}=-$ $78\left(c 0.05, \mathrm{CHCl}_{3}\right) ;{ }^{1} \mathrm{H} \mathrm{NMR}\left(400 \mathrm{MHz}, \mathrm{CDCl}_{3}\right) \delta 7.68(\mathrm{~d}, J=8.0 \mathrm{~Hz}, 2 \mathrm{H})$, 7.40-7.28 (m, 3H), $7.23(\mathrm{~d}, J=6.7 \mathrm{~Hz}, 2 \mathrm{H}), 7.03(\mathrm{~d}, J=7.9 \mathrm{~Hz}, 2 \mathrm{H}), 5.82(\mathrm{dd}, J=10.6,5.7 \mathrm{~Hz}$, $1 \mathrm{H}), 5.41(\mathrm{~d}, J=10.2 \mathrm{~Hz}, 1 \mathrm{H}), 4.87(\mathrm{~s}, 1 \mathrm{H}), 4.44-4.32(\mathrm{~m}, 2 \mathrm{H}), 4.23-4.18(\mathrm{~m}, 1 \mathrm{H}), 3.61(\mathrm{~d}, J=$ $9.0 \mathrm{~Hz}, 1 \mathrm{H}), 3.46-3.33(\mathrm{~m}, 2 \mathrm{H}), 3.16(\mathrm{td}, J=12.2,5.0 \mathrm{~Hz}, 1 \mathrm{H}), 2.98(\mathrm{~d}, J=18.8 \mathrm{~Hz}, 1 \mathrm{H}), 2.50$ $(\mathrm{dd}, J=18.9,5.9 \mathrm{~Hz}, 1 \mathrm{H}), 2.33(\mathrm{~s}, 3 \mathrm{H}), 1.45(\mathrm{td}, J=12.2,7.6 \mathrm{~Hz}, 1 \mathrm{H}), 1.24$ (dd, $J=12.2,4.9$ $\mathrm{Hz}, 1 \mathrm{H}) ;{ }^{13} \mathrm{C} \mathrm{NMR}\left(100 \mathrm{MHz}, \mathrm{CDCl}_{3}\right) \delta 143.6,138.2,134.3,129.6,128.3,128.3,127.9,127.7$, 125.3, 73.7, 73.2, 61.9, 48.0, 47.7, 47.2, 34.7, 29.0, 21.6; IR (KBr, $\left.\mathrm{cm}^{-1}\right)$ 2976, 1654, 1597, 1493, 1397, 1359, 1248, 1158, 1032, 996, 813, 784, 739, 701, 662; HRMS $(\mathrm{M}+\mathrm{Na})^{+}$calcd for $\mathrm{C}_{23} \mathrm{H}_{26} \mathrm{BrNNaO}_{3} \mathrm{~S}$ 498.0714; found 498.0725; HPLC conditions: Daicel Chiralcel OD-H, Hexane $/ i-\mathrm{PrOH}=90: 10,1.0 \mathrm{~mL} / \mathrm{min}, \lambda=254 \mathrm{~nm}, t_{1}=9.2 \mathrm{~min}$ (minor), $t_{2}=10.3 \mathrm{~min}$ (major). 
(3a $R, 7 S, 7 \mathrm{a} S)-7-b r o m o-3 a-(((t e r t-b u t y l d i m e t h y l s i l y l) o x y) m e t h y l)-1-t o s y l-2,3,3 a, 6,7,7 a-$

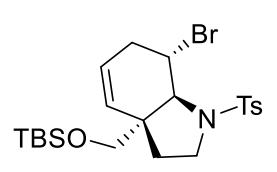

hexahydro-1H-indole (2q): flash column chromatography (petroleum ether / EtOAc = 15:1), $29.6 \mathrm{mg}, 59 \%$ yield, $95 \%$ ee, white solid, $\mathrm{mp}=141-143{ }^{\circ} \mathrm{C}$; $[\alpha]_{\mathrm{D}}{ }^{20}=-99\left(c 0.31, \mathrm{CHCl}_{3}\right) ;{ }^{1} \mathrm{H}$ NMR $\left(400 \mathrm{MHz}, \mathrm{CDCl}_{3}\right) \delta 7.74(\mathrm{~d}, J=7.9$ $\mathrm{Hz}, 2 \mathrm{H}), 7.32(\mathrm{~d}, J=7.9 \mathrm{~Hz}, 2 \mathrm{H}), 5.81(\mathrm{dd}, J=10.7,5.5 \mathrm{~Hz}, 1 \mathrm{H}), 5.45(\mathrm{~d}, J=10.2 \mathrm{~Hz}, 1 \mathrm{H})$, $4.92(\mathrm{~s}, 1 \mathrm{H}), 3.93(\mathrm{~d}, J=3.6 \mathrm{~Hz}, 1 \mathrm{H}), 3.66-3.55$ (m, 2H), 3.44 (dd, $J=11.6,7.7 \mathrm{~Hz}, 1 \mathrm{H}), 3.21$ $(\operatorname{td}, J=11.9,5.2 \mathrm{~Hz}, 1 \mathrm{H}), 2.99(\mathrm{~d}, J=18.8 \mathrm{~Hz}, 1 \mathrm{H}), 2.55-2.35(\mathrm{~m}, 4 \mathrm{H}), 1.50(\mathrm{td}, J=12.1,7.7$ $\mathrm{Hz}, 1 \mathrm{H}), 1.29(\mathrm{dd}, J=12.3,5.1 \mathrm{~Hz}, 1 \mathrm{H}), 0.79(\mathrm{~s}, 9 \mathrm{H}),-0.06(\mathrm{~d}, J=6.2 \mathrm{~Hz}, 6 \mathrm{H}) ;{ }^{13} \mathrm{C} \mathrm{NMR}(100$ $\left.\mathrm{MHz}, \mathrm{CDCl}_{3}\right) \delta 143.7,134.7,129.9,128.4,127.5,125.0,67.1,61.6,49.2,47.5,47.3,34.3,29.2$, 25.8, 21.6, 18.4, -5.4, -5.5; IR (KBr, cm $\left.{ }^{-1}\right)$ 2954, 1699, 1600, 1468, 1398, 1348, 1248, 1160 , 1029, 935, 810, 787, 730, 705, 666; HRMS (M+Na) ${ }^{+}$calcd for $\mathrm{C}_{22} \mathrm{H}_{34} \mathrm{BrNNaO}_{3} \mathrm{SSi}$ 522.1110; found 522.1122; HPLC conditions: Daicel Chiralcel OD-H, Hexane $/ i-\mathrm{PrOH}=95: 5,1.0 \mathrm{~mL} / \mathrm{min}$, $\lambda=254 \mathrm{~nm}, t_{1}=5.6 \mathrm{~min}$ (major), $t_{2}=6.9 \mathrm{~min}$ (minor).

(3aS,7S,7aS)-3a-benzyl-7-bromo-1-tosyl-2,3,3a,6,7,7a-hexahydro-1H-indole (2r): flash column chromatography (petroleum ether / EtOAc $=15: 1$ ), $33.9 \mathrm{mg}, 76 \%$ yield, Br $93 \%$ ee, white solid, $\mathrm{mp}=167-170{ }^{\circ} \mathrm{C} ;[\alpha]_{\mathrm{D}}{ }^{20}=-65\left(c 0.32, \mathrm{CHCl}_{3}\right) ;{ }^{1} \mathrm{H} \mathrm{NMR}$ $\left(400 \mathrm{MHz}, \mathrm{CDCl}_{3}\right) \delta 7.45(\mathrm{~d}, J=7.9 \mathrm{~Hz}, 2 \mathrm{H}), 7.29-7.19$ (m, 3H), 7.16-7.05 (m, $4 \mathrm{H}), 5.81(\mathrm{dd}, J=10.6,5.6 \mathrm{~Hz}, 1 \mathrm{H}), 5.42(\mathrm{~d}, J=9.3 \mathrm{~Hz}, 1 \mathrm{H}), 4.94(\mathrm{~s}, 1 \mathrm{H}), 3.94$ $(\mathrm{d}, J=3.7 \mathrm{~Hz}, 1 \mathrm{H}), 3.37-3.26(\mathrm{~m}, 1 \mathrm{H}), 3.16-3.07(\mathrm{~m}, 1 \mathrm{H}), 3.06-2.92(\mathrm{~m}, 3 \mathrm{H}), 2.52(\mathrm{dd}, J=18.9$, $5.7 \mathrm{~Hz}, 1 \mathrm{H}), 2.39$ (s, 3H), 1.24-1.14 (m, 2H); ${ }^{13} \mathrm{C} \mathrm{NMR}\left(100 \mathrm{MHz}, \mathrm{CDCl}_{3}\right) \delta 143.3,136.7$, 134.2, 131.2, 131.1, 129.8, 128.0, 127.1, 126.4, 123.9, 63.1, 47.6, 47.0, 46.8, 43.8, 35.7, 29.0, 21.5; IR (KBr, cm $\left.{ }^{-1}\right)$ 2962, 1700, 1599, 1452, 1397, 1240, 1156, 1021, 935, 812, 781, 731, 704, 661; HRMS (M+Na) ${ }^{+}$calcd for $\mathrm{C}_{22} \mathrm{H}_{24} \mathrm{BrNO}_{2} \mathrm{~S}$ 468.0609; found 468.0601; HPLC conditions: Daicel Chiralcel OD-H, Hexane $/ i-\mathrm{PrOH}=90: 10,1.0 \mathrm{~mL} / \mathrm{min}, \lambda=254 \mathrm{~nm}, t_{1}=9.7 \mathrm{~min}$ (major), $t_{2}=10.4 \min$ (minor).

(3aS,7S,7aS)-7-bromo-3a-phenyl-3a,6,7,7a-tetrahydrobenzofuran-2(3H)-one (2s): flash

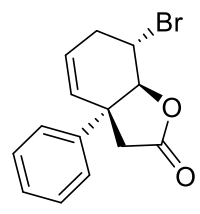
column chromatography (petroleum ether $/$ EtOAc $=20: 1$ ), $19.6 \mathrm{mg}, 67 \%$ yield, $10 \%$ ee, white solid, $\mathrm{mp}=132-134{ }^{\circ} \mathrm{C} ;[\alpha]_{\mathrm{D}}{ }^{20}=-41\left(c 0.08, \mathrm{CHCl}_{3}\right) ;{ }^{1} \mathrm{H} \mathrm{NMR}$ $\left(400 \mathrm{MHz}, \mathrm{CDCl}_{3}\right) \delta$ 7.44-7.22 (m, 5H), 6.03-5.87 (m, 2H), $4.84(\mathrm{~d}, J=7.4 \mathrm{~Hz}$, $1 \mathrm{H}), 4.35-4.25$ (m, 1H), 3.15 (d, $J=17.7 \mathrm{~Hz}, 1 \mathrm{H}), 2.91$ (dt, $J=18.3,4.6 \mathrm{~Hz}, 1 \mathrm{H})$, $2.79(\mathrm{~d}, J=17.8 \mathrm{~Hz}, 1 \mathrm{H}), 2.74-2.61(\mathrm{~m}, 1 \mathrm{H}) ;{ }^{13} \mathrm{C} \mathrm{NMR}\left(100 \mathrm{MHz}, \mathrm{CDCl}_{3}\right) \delta 174.0,142.4$, 
130.0, 128.9, 127.6, 126.3, 125.0, 87.2, 47.8, 44.1, 42.8, 31.2; IR (KBr, cm $\left.{ }^{-1}\right) 3533,2974,1778$, $1685,1596,1493,1419,1323,1270,1181,1088,946,830,771,721,701,662$; HRMS $(\mathrm{M}+\mathrm{Na})^{+}$calcd for $\mathrm{C}_{14} \mathrm{H}_{13} \mathrm{BrNaO}_{2}$ 314.9997; found 314.9987; HPLC conditions: Daicel Chiralcel OD-H, Hexane $/ i-\mathrm{PrOH}=90: 10,1.0 \mathrm{~mL} / \mathrm{min}, \lambda=254 \mathrm{~nm}, t_{1}=20.7 \mathrm{~min}$ (major), $t_{2}=$ 25.3 min (minor).

\section{Demonstration of Synthetic Utility.}

\section{a) Transformation of $2 a^{[4,5]}$}
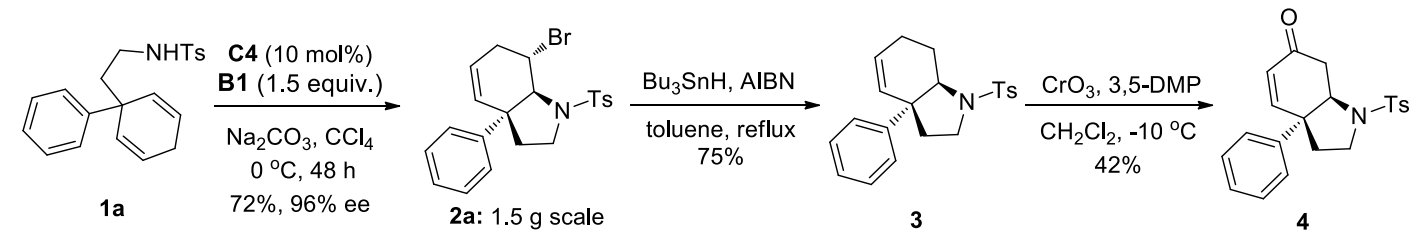

To a flame-dried 2-dram vial was added (R)-TRIP C4 (37.6 mg, $0.05 \mathrm{mmol}, 10 \mathrm{~mol} \%)$, brominating agent $\mathbf{B 1}$ (495 mg, $0.75 \mathrm{mmol}, 1.5$ equiv.), $\mathrm{Na}_{2} \mathrm{CO}_{3}(210 \mathrm{mg}, 2.0 \mathrm{mmol}, 4.0$ equiv.) and $\mathrm{CCl}_{4}(5.0 \mathrm{~mL})$, the mixture was stirred at $0^{\circ} \mathrm{C}$ for $10 \mathrm{~min}$., then the solution of substrate $(1.765 \mathrm{~g}, 0.5 \mathrm{mmol})$ in $\mathrm{CCl}_{4}(5.0 \mathrm{~mL})$ was added. The reaction was stirred at $0^{\circ} \mathrm{C}$ for $48 \mathrm{~h}$. Upon completion, the reaction was quenched with saturated $\mathrm{Na}_{2} \mathrm{~S}_{2} \mathrm{O}_{3}$ and then was extracted with $\mathrm{CH}_{2} \mathrm{Cl}_{2}$. The combined extracts were washed with brine $(50.0 \mathrm{~mL})$, dried over $\mathrm{Na}_{2} \mathrm{SO}_{4}$ and concentrated in vacuo. The residue was purified by flash column chromatography (petro ether/EtOAc $=15 / 1)$ to yield the product $2 \mathbf{a}(1.56 \mathrm{~g}, 72 \%$ yield $)$.

A solution of $\mathbf{2 a}(860 \mathrm{mg}, 2 \mathrm{mmol}), \mathrm{Bu}_{3} \mathrm{SnH}(5.4 \mathrm{~mL}, 20 \mathrm{mmol})$, and AIBN (330 mg, 2 $\mathrm{mmol})$ in benzene $(25 \mathrm{~mL})$ was heated under reflux under an inert atmosphere for 2 hours. After the disappearance of the starting material (TLC monitoring), the benzene was removed under vacuum and the residue was purified by flash chromatogramphy $(\mathrm{PE} / \mathrm{EtOAc}=5 / 1)$ to give the products 3 (530 $\mathrm{mg}, 75 \%$ yield).

A cooled $\left(-10^{\circ} \mathrm{C}\right)$ solution of chromium trioxide $\left(5 \mathrm{~g}, 50 \mathrm{mmol}, 10\right.$ equiv) in $\mathrm{CH}_{2} \mathrm{Cl}_{2}(20$ $\mathrm{mL})$ was treated with 3,5-dimethylpyrazole (4.81 g, $50 \mathrm{mmol}, 10$ equiv) and the reaction mixture was allowed to stir for $30 \mathrm{~min}$ at $-10{ }^{\circ} \mathrm{C}$ before $3(1.77 \mathrm{~g}, 5 \mathrm{mmol})$ was added. After stirring for $16 \mathrm{~h}$ at $25{ }^{\circ} \mathrm{C}$, the reaction mixture was diluted with $\mathrm{Et}_{2} \mathrm{O}$ and stirred for additional 
$30 \mathrm{~min}$ at the same temperature. The resulting mixture was then filtered through celite and concentrated. The residue was purified by column chromatography $(\mathrm{PE} / \mathrm{EtOAc}=2 / 1)$ to provide the product 4 ( $0.763 \mathrm{~g}, 42 \%$ yield $)$.

(3aS,7aR)-3a-phenyl-1-tosyl-2,3,3a,6,7,7a-hexahydro-1H-indole $\quad(3):$ flash column

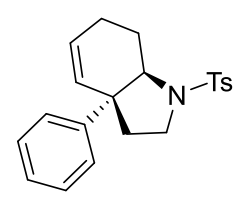
chromatography (petroleum ether / EtOAc $=5: 1$ ), $530 \mathrm{mg}, 75 \%$ yield, white solid, mp $=150-153{ }^{\circ} \mathrm{C} ;{ }^{1} \mathrm{H}$ NMR $\left(400 \mathrm{MHz}, \mathrm{CDCl}_{3}\right) \delta 7.70(\mathrm{~d}, J=8.0 \mathrm{~Hz}$, 2H), $7.29(\mathrm{~d}, J=7.9 \mathrm{~Hz}, 2 \mathrm{H}), 7.23-7.12(\mathrm{~m}, 3 \mathrm{H}), 7.02(\mathrm{~d}, J=7.6 \mathrm{~Hz}, 2 \mathrm{H})$, 6.10-6.01 (m, 1H), $5.46(\mathrm{~d}, J=10.1 \mathrm{~Hz}, 1 \mathrm{H}), 3.76-3.69(\mathrm{~m}, 1 \mathrm{H}), 3.66-3.57$ (m, 1H), 3.45 (td, $J$ $=10.9,5.6 \mathrm{~Hz}, 1 \mathrm{H}), 2.45(\mathrm{~s}, 3 \mathrm{H}), 2.39-2.27(\mathrm{~m}, 1 \mathrm{H}), 2.18-2.09(\mathrm{~m}, 1 \mathrm{H}), 2.07-1.95(\mathrm{~m}, 1 \mathrm{H})$, $1.88(\mathrm{q}, J=11.1 \mathrm{~Hz}, 1 \mathrm{H}), 1.70-1.60(\mathrm{~m}, 2 \mathrm{H}) ;{ }^{13} \mathrm{C} \mathrm{NMR}\left(100 \mathrm{MHz}, \mathrm{CDCl}_{3}\right) \delta 143.4,143.3$, 135.0, 130.2, 129.8, 129.6, 128.3, 127.4, 126.7, 126.6, 65.9, 50.8, 48.2, 36.6, 24.0, 21.5, 20.1. HRMS $(\mathrm{M}+\mathrm{Na})^{+}$calcd for $\mathrm{C}_{21} \mathrm{H}_{23} \mathrm{NNaO}_{2} \mathrm{~S} 376.1347$; found 376.1342 .

(3aS,7aR)-3a-phenyl-1-tosyl-1,2,3,3a,7,7a-hexahydro-6H-indol-6-one (4): flash column

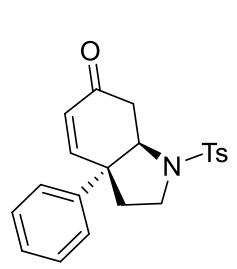
chromatography (petroleum ether / EtOAc $=2: 1$ ), $0.763 \mathrm{~g}, 42 \%$ yield, white solid, $\mathrm{mp}=156-157{ }^{\circ} \mathrm{C} ;{ }^{1} \mathrm{H} \mathrm{NMR}\left(400 \mathrm{MHz}, \mathrm{CDCl}_{3}\right) \delta 7.69(\mathrm{~d}, J=7.9 \mathrm{~Hz}, 2 \mathrm{H})$, 7.35-7.26 (m, 5H), 7.15-7.07 (m, 2H), $6.63(\mathrm{~d}, J=10.2 \mathrm{~Hz}, 1 \mathrm{H}), 6.27$ (d, $J=$ $10.2 \mathrm{~Hz}, 1 \mathrm{H}), 4.16-4.09(\mathrm{~m}, 1 \mathrm{H}), 3.73(\mathrm{t}, J=8.8 \mathrm{~Hz}, 1 \mathrm{H}), 3.33(\mathrm{td}, J=11.4,5.6$ $\mathrm{Hz}, 1 \mathrm{H}), 3.13(\mathrm{dd}, J=17.1,3.1 \mathrm{~Hz}, 1 \mathrm{H}), 2.66-2.57$ (m, 1H), 2.45 (s, 3H), 2.22$2.10(\mathrm{~m}, 1 \mathrm{H}), 1.95(\mathrm{dd}, J=12.2,4.0 \mathrm{~Hz}, 1 \mathrm{H}) ;{ }^{13} \mathrm{C} \mathrm{NMR}\left(100 \mathrm{MHz}, \mathrm{CDCl}_{3}\right) \delta 196.4,149.7$, 143.9, 138.8, 134.9, 130.7, 129.8, 129.1, 127.9, 127.5, 126.3, 65.7, 51.6, 48.0, 39.2, 37.1, 21.6. HRMS $(\mathrm{M}+\mathrm{Na})^{+}$calcd for $\mathrm{C}_{21} \mathrm{H}_{21} \mathrm{NNaO}_{3} \mathrm{~S} 390.1140$; found 390.1142 .

b) The total synthesis of (+)-Mesembrane. ${ }^{[1,, 4,6]}$ 


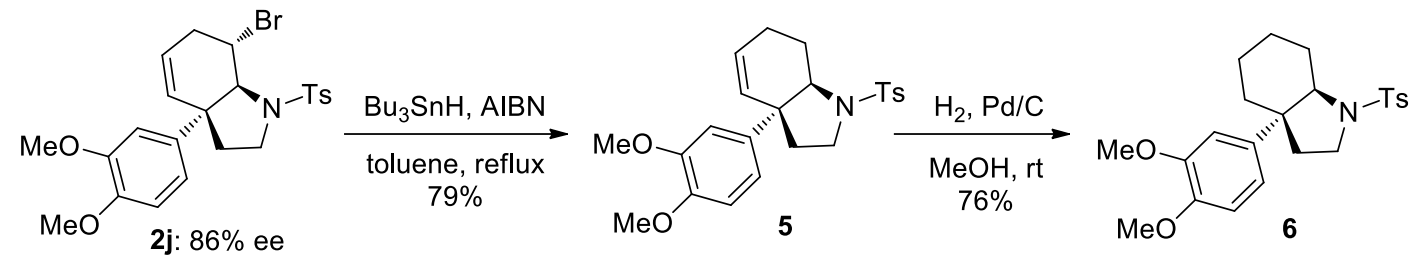
$>98 \%$ ee after recrystallization

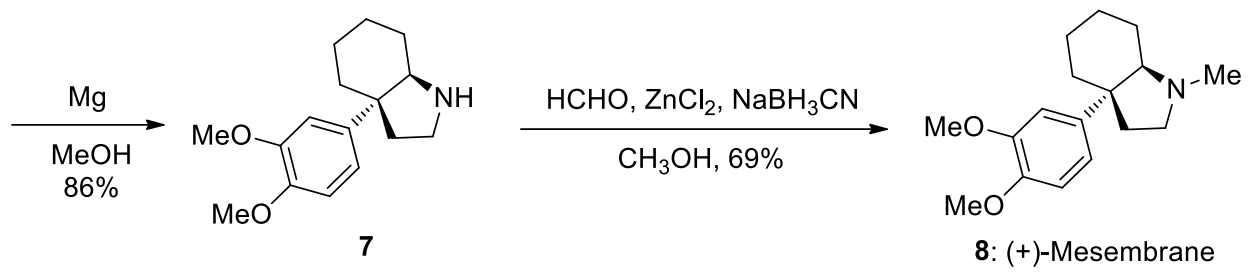

A solution of $\mathbf{2 j}$ (700 mg, $1.4 \mathrm{mmol}), \mathrm{Bu}_{3} \mathrm{SnH}$ (1.9 mL, $7 \mathrm{mmol}$ ), and AIBN (276 mg, 1.68 mmol) in toluene $(17.5 \mathrm{~mL})$ was heated under reflux under an inert atmosphere for 2 hours. After the disappearance of the starting material (TLC monitoring), the toluene was removed under vacuum and the residue was purified by flash chromatogramphy (PE/EtOAc:5/1) to give the product 5 (457 $\mathrm{mg}, 79 \%$ yield).

A mixture of 5 (331 mg, $0.8 \mathrm{mmol})$ and 10\% $\mathrm{Pd} / \mathrm{C}(33 \mathrm{mg})$ in $\mathrm{MeOH}(8 \mathrm{~mL})$ was stirred under $\mathrm{H}_{2}$ atmosphere. After the disappearance of the starting material (TLC monitoring), the mixture was filtered, $\mathrm{MeOH}$ was removed under vacuum and the residue was purified by flash chromatogramphy (PE/EtOAc: 5/1) to give the product 6 (253 mg, 76\% yield).

Magnesium (pre-activated by $1 \mathrm{M} \mathrm{HCl}$ aq.) (340 mg, $14 \mathrm{mmol}$ ) was added to a solution of 6 (291 mg, $0.7 \mathrm{mmol})$ in $\mathrm{MeOH}(7 \mathrm{~mL})$ and stirred vigorously at room temperature for 12 hours. Reaction was quenched by $1 \mathrm{M} \mathrm{HCl}$ aq. and basified with saturated $\mathrm{NaHCO}_{3}$ aq. Organic layer was extracted with EtOAc, washed with brine, and dried over $\mathrm{Na}_{2} \mathrm{SO}_{4}$. The solvent was removed under vacuum and the residue was purified by silica gel column chromatography $\left(\mathrm{MeOH} / \mathrm{CH}_{2} \mathrm{Cl}_{2}: 1 / 10\right)$ to give the product 7 (157 $\mathrm{mg}, 86 \%$ yield).

To a solution of 7 (52 mg, $0.2 \mathrm{mmol})$ in $\mathrm{MeOH}(5 \mathrm{~mL})$ was added 37\% aqueous $\mathrm{HCHO}$ (30 mg, $1 \mathrm{mmol}), \mathrm{ZnCl}_{2}(16 \mathrm{mg}, 0.12 \mathrm{mmol})$ and $\mathrm{NaBH}_{3} \mathrm{CN}(26 \mathrm{mg}, 0.4 \mathrm{mmol})$ at room temperature. The reaction was stirred for 30 min and quenched with $0.1 \mathrm{~N} \mathrm{NaOH}(2 \mathrm{~mL})$. Methanol was removed under reduced pressure and the residue was extracted with EtOAc. The organic layer was washed with brine, dried over anhydrous $\mathrm{Na}_{2} \mathrm{SO}_{4}$. The solvent was removed 
and the residue was purified by silica gel column chromatography $\left(\mathrm{CH}_{2} \mathrm{Cl}_{2} / \mathrm{MeOH}=20 / 1\right)$ to provide (+)-Mesembrane 8 (38 $\mathrm{mg}, 69 \%$ yield).

(3aS,7aR)-3a-(3,4-dimethoxyphenyl)-1-tosyl-2,3,3a,6,7,7a-hexahydro-1H-indole (5): flash

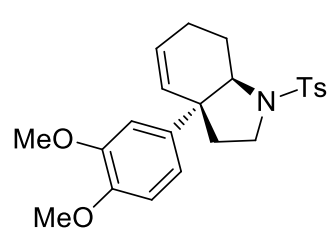

column chromatography (petroleum ether $/$ EtOAc $=5: 1$ ), 79\% yield, colorless oil; ${ }^{1} \mathrm{H}$ NMR (400 MHz, $\left.\mathrm{CDCl}_{3}\right) \delta 7.70(\mathrm{~d}, J=8.1 \mathrm{~Hz}, 2 \mathrm{H}), 7.28$ $(\mathrm{d}, J=7.9 \mathrm{~Hz}, 2 \mathrm{H}), 6.69(\mathrm{~d}, J=8.4 \mathrm{~Hz}, 1 \mathrm{H}), 6.62(\mathrm{dd}, J=8.4,1.9 \mathrm{~Hz}, 1 \mathrm{H})$, $6.47(\mathrm{~d}, J=1.7 \mathrm{~Hz}, 1 \mathrm{H}), 6.07-6.00(\mathrm{~m}, 1 \mathrm{H}), 5.43(\mathrm{~d}, J=10.1 \mathrm{~Hz}, 1 \mathrm{H}), 3.83$ (s, 3H), $3.72(\mathrm{~s}, 3 \mathrm{H}), 3.69-3.58(\mathrm{~m}, 2 \mathrm{H}), 3.45(\mathrm{td}, J=10.9,5.6 \mathrm{~Hz}, 1 \mathrm{H}), 2.43(\mathrm{~s}, 3 \mathrm{H}), 2.39-2.28$ (m, 1H), 2.17-2.08 (m, 1H), 2.06-1.95 (m, 1H), 1.90-1.80 (m, 1H), 1.65-1.59 (m, 2H); ${ }^{13} \mathrm{C} \mathrm{NMR}$ $\left(100 \mathrm{MHz}, \mathrm{CDCl}_{3}\right) \delta 148.7,147.8,143.2,135.8,135.0,130.2,129.8,129.5,127.4,119.3,110.8$, 109.9, 66.0, 55.8, 55.7, 50.6, 48.2, 36.7, 24.0, 21.5, 20.2. HRMS $(\mathrm{M}+\mathrm{Na})^{+}$calcd for $\mathrm{C}_{23} \mathrm{H}_{27} \mathrm{NNaO}_{4} \mathrm{~S} 436.1558$; found 436.1549 .

(3a $R, 7 \mathrm{a} R)$-3a-(3,4-dimethoxyphenyl)-1-tosyloctahydro-1H-indole $\quad(6):$ flash column

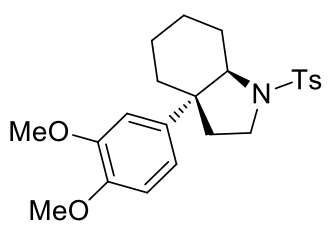
chromatography (petroleum ether / EtOAc $=5: 1$ ), 76\% yield, colorless oil; ${ }^{1} \mathrm{H}$ NMR $\left(400 \mathrm{MHz}, \mathrm{CDCl}_{3}\right) \delta 7.48(\mathrm{~d}, J=8.1 \mathrm{~Hz}, 2 \mathrm{H}), 7.11(\mathrm{~d}, J=8.0$ $\mathrm{Hz}, 2 \mathrm{H}), 6.68(\mathrm{dd}, J=8.4,2.0 \mathrm{~Hz}, 1 \mathrm{H}), 6.65-6.59(\mathrm{~m}, 2 \mathrm{H}), 3.92-3.86(\mathrm{~m}$, 1H), $3.83(\mathrm{~s}, 3 \mathrm{H}), 3.79(\mathrm{~s}, 3 \mathrm{H}), 3.58-3.50(\mathrm{~m}, 1 \mathrm{H}), 3.34-3.24(\mathrm{~m}, 1 \mathrm{H}), 2.37$ (s, 3H), 2.21-2.12 (m, 1H), 2.04-1.97 (m, 1H), 1.92-1.83 (m, 2H), 1.82-1.78 (m, 1H), 1.78-1.66 (m, 2H), 1.48-1.40 (m, 2H), 1.39-1.29 (m, 1H); $\left.{ }^{13} \mathrm{C} \mathrm{NMR} \mathrm{(100} \mathrm{MHz,} \mathrm{CDCl}_{3}\right) \delta 148.5,147.4$, 142.7, 138.0, 135.5, 129.1, 126.9, 117.8, 110.5, 109.6, 64.0, 55.7, 48.3, 45.7, 35.5, 33.7, 30.0, 22.4, 22.0, 21.3. HRMS (M+Na) ${ }^{+}$calcd for $\mathrm{C}_{23} \mathrm{H}_{29} \mathrm{NNaO}_{4} \mathrm{~S} 438.1715$; found 438.1706.

(3aR,7aR)-3a-(3,4-dimethoxyphenyl)-1-methyloctahydro-1H-indole (8) : flash column

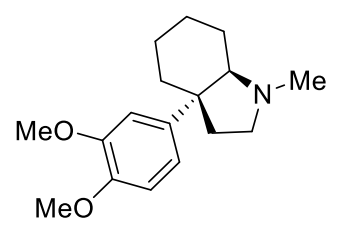
chromatography $\left(\mathrm{CH}_{2} \mathrm{Cl}_{2} / \mathrm{MeOH}=20 / 1\right), 38 \mathrm{mg}, 69 \%$ yield, colorless oil; $[\alpha]_{\mathrm{D}}{ }^{30}=+12\left(c 0.38, \mathrm{CHCl}_{3}\right) ;{ }^{1} \mathrm{H}$ NMR $\left(400 \mathrm{MHz}, \mathrm{CDCl}_{3}\right) \delta 6.93-6.84(\mathrm{~m}$, $2 \mathrm{H}), 6.80(\mathrm{~d}, J=8.3 \mathrm{~Hz}, 1 \mathrm{H}), 3.87(\mathrm{~s}, 3 \mathrm{H}), 3.85(\mathrm{~s}, 3 \mathrm{H}), 3.30(\mathrm{td}, J=9.2$, $4.9 \mathrm{~Hz}, 1 \mathrm{H}), 2.65$ (s, 1H), 2.40-2.29 (m, 4H), 1.97-1.76 (m, 5H), 1.65-1.56 
$(\mathrm{m}, 2 \mathrm{H}), 1.52-1.44(\mathrm{~m}, 1 \mathrm{H}), 1.41-1.33(\mathrm{~m}, 1 \mathrm{H}), 1.20-1.08(\mathrm{~m}, 1 \mathrm{H}) ;{ }^{13} \mathrm{C} \mathrm{NMR}\left(100 \mathrm{MHz}, \mathrm{CDCl}_{3}\right)$ $\delta$ 148.6, 146.9, 139.8, 118.7, 110.7, 110.7, 68.8, 55.9, 55.8, 54.2, 47.5, 40.8, 40.7, 35.8, 23.5, 22.7, 20.3. HRMS (M+Na) ${ }^{+}$calcd for $\mathrm{C}_{17} \mathrm{H}_{25} \mathrm{NNaO}_{2} 298.1783$; found 298.1788.

\section{References}

[1] (a) Lebeuf, R.; Dunet, J.; Beniazza, R.; Ibrahim, D.; Bose, G.; Berlande, M.; Robert, F.; Landais, Y. Birch Reductive Alkylation of Biaryls: Scope and Limitations. J. Org.Chem. 2009, 74, 6469-6478. (b) Lebeuf, R.; Robert, F.; Landais, Y. Regioselectivity of Birch Reductive Alkylation of Biaryls. Org. Lett. 2005, 7, 4557-4560. (c) Bao, X.; Wang, Q.; Zhu, J. Palladium-Catalyzed Enantioselective Desymmetrizing Aza-Wacker Reaction: Development and Application to the Total Synthesis of (-)-Mesembrane and (+)-Crinane . Angew. Chem. Int. Ed. 2018, 57, 1995-1999.

[2] Hanley, P. S.; Ober, M. S.; Krasovskiy, A. L.; Whiteker, G. T.; Kruper, W. J. Nickel- and Palladium-Catalyzed Coupling of Aryl Fluorosulfonates with Aryl Boronic Acids Enabled by Sulfuryl Fluoride. ACS Catal. 2015, 5, 5041-5046.

[3] (a) Chen, W.; Oestreich, M. Metal-Free Transfer Hydrobromination of C-Ctriple Bonds. Org. Lett. 2019, 21, 4531-4534; (b) Hampel, T.; Bruckner, R. A Novel Cis-Selective Cyclohexanone Annulation as the Key Step of a Total Synthesis of the Sesquiterpene Isoacanthodoral. Org. Lett. 2009, 11, 4842-4845.

[4] Yu, S.; Li, Y.; Deng, J. Enantioselective Synthesis of 2-Bromomethyl Indolines via BINAP(S)-Catalyzed Bromoaminocyclization of Allyl Aniline. Adv. Synth. Catal. 2017, $359,2499-2508$.

[5] (a) Salmond, W. G.; Barta, M. A.; Havens, J. L. Allylic Oxidation with 3,5Dimethylpyrazole. Chromium Trioxide Complex Steroidal . $\Delta^{5}-7$-ketones. J. Org. Chem. 1978, 43, 2057-2059. (b) Hayashi, K.; Tanimoto, H.; Zhang, H.; Morimoto, T.; Nishiyama, Y.; Kakiuchi, K. Efficient Synthesis of $\alpha, \beta$-Unsaturated Alkylimines Performed with Allyl Cations and Azides: Application to the Synthesis of an Ant Venom Alkaloid. Org. Lett. 2012, $14,5728-5731$. 
[6] Kishi, K.; Takizawa, S.; Sasai, H. Phosphine-Catalyzed Dual Umpolung Domino Michael Reaction: Facile Synthesis of Hydroindole- and Hydrobenzofuran-2- Carboxylates. ACS Catal. 2018, 8, 5228-5232. 


\section{Copies of NMR Spectra}

${ }^{1} \mathrm{H}$ NMR of $1 \mathrm{a}\left(400 \mathrm{MHz}, \mathrm{CDCl}_{3}\right)$

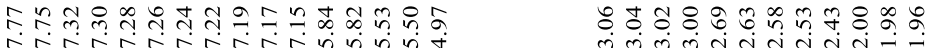

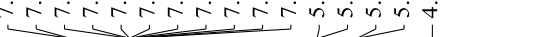

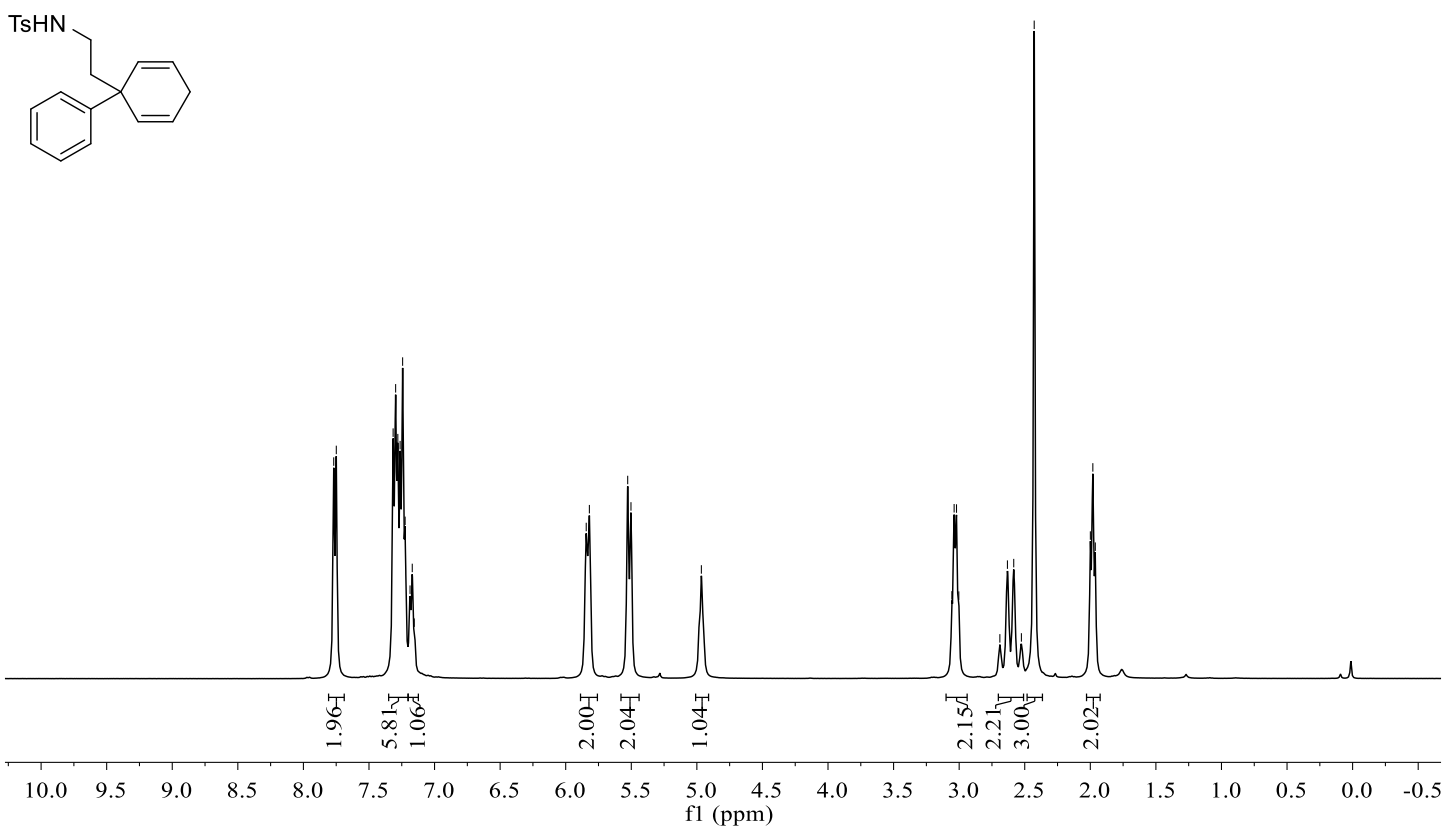

${ }^{13} \mathrm{C}$ NMR of $1 \mathrm{a}\left(100 \mathrm{MHz}, \mathrm{CDCl}_{3}\right)$

舟品

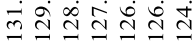

궁

산

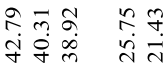

i)
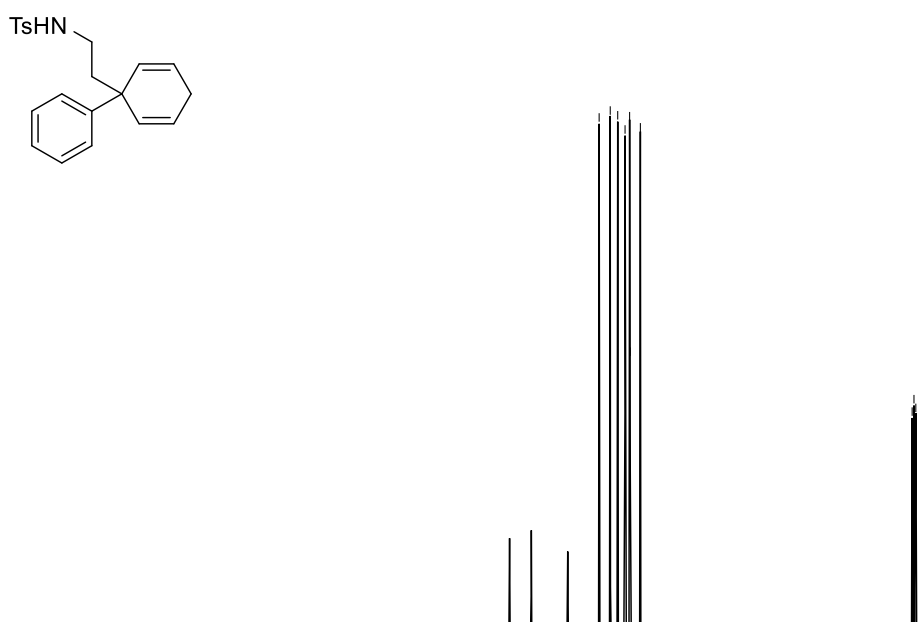

$\begin{array}{lllllllllllllllllllllllll}230 & 220 & 210 & 200 & 190 & 180 & 170 & 160 & 150 & 140 & 130 & 120 & 110 & 100 & 90 & 80 & 70 & 60 & 50 & 40 & 30 & 20 & 10 & 0 & -10\end{array}$ f1 (ppm) 
${ }^{1} \mathrm{H}$ NMR of $1 \mathrm{~b}\left(400 \mathrm{MHz}, \mathrm{CDCl}_{3}\right)$

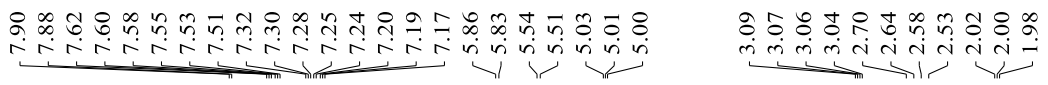

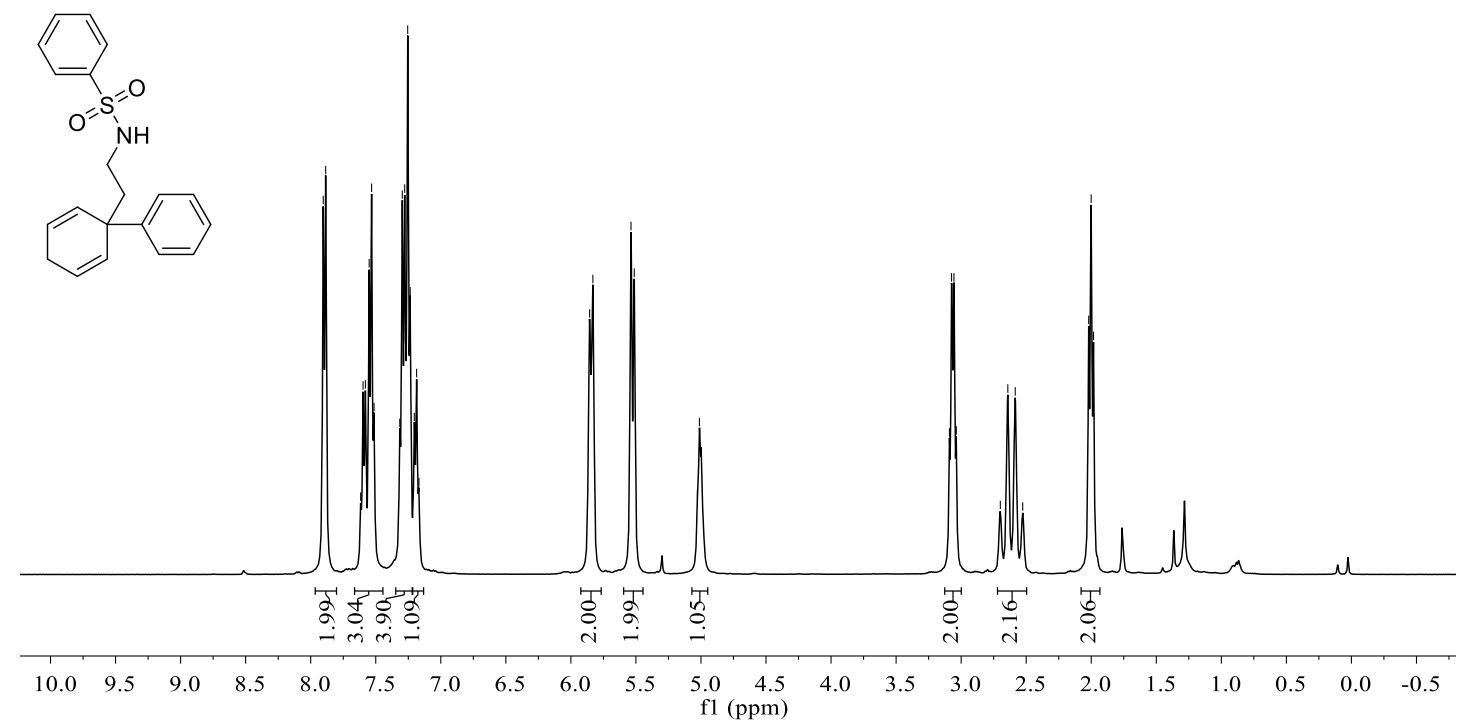

${ }^{13} \mathrm{C}$ NMR of $1 \mathrm{~b}\left(100 \mathrm{MHz}, \mathrm{CDCl}_{3}\right)$

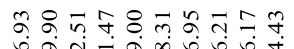

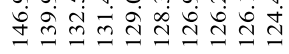

致

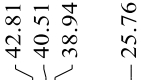

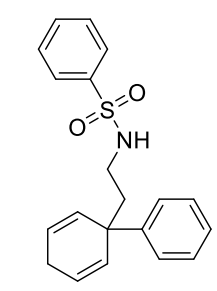

$\begin{array}{rllllllllllll}230 & 220 & 210 & 200 & 190 & 180 & 170 & 160 & 150 & 140 & 130 & 120 & 110 \\ \mathrm{f} 1(\mathrm{ppm})\end{array}$ 
${ }^{1} \mathrm{H}$ NMR of $1 \mathrm{c}\left(400 \mathrm{MHz}, \mathrm{CDCl}_{3}\right)$

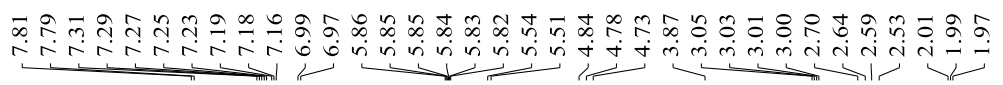
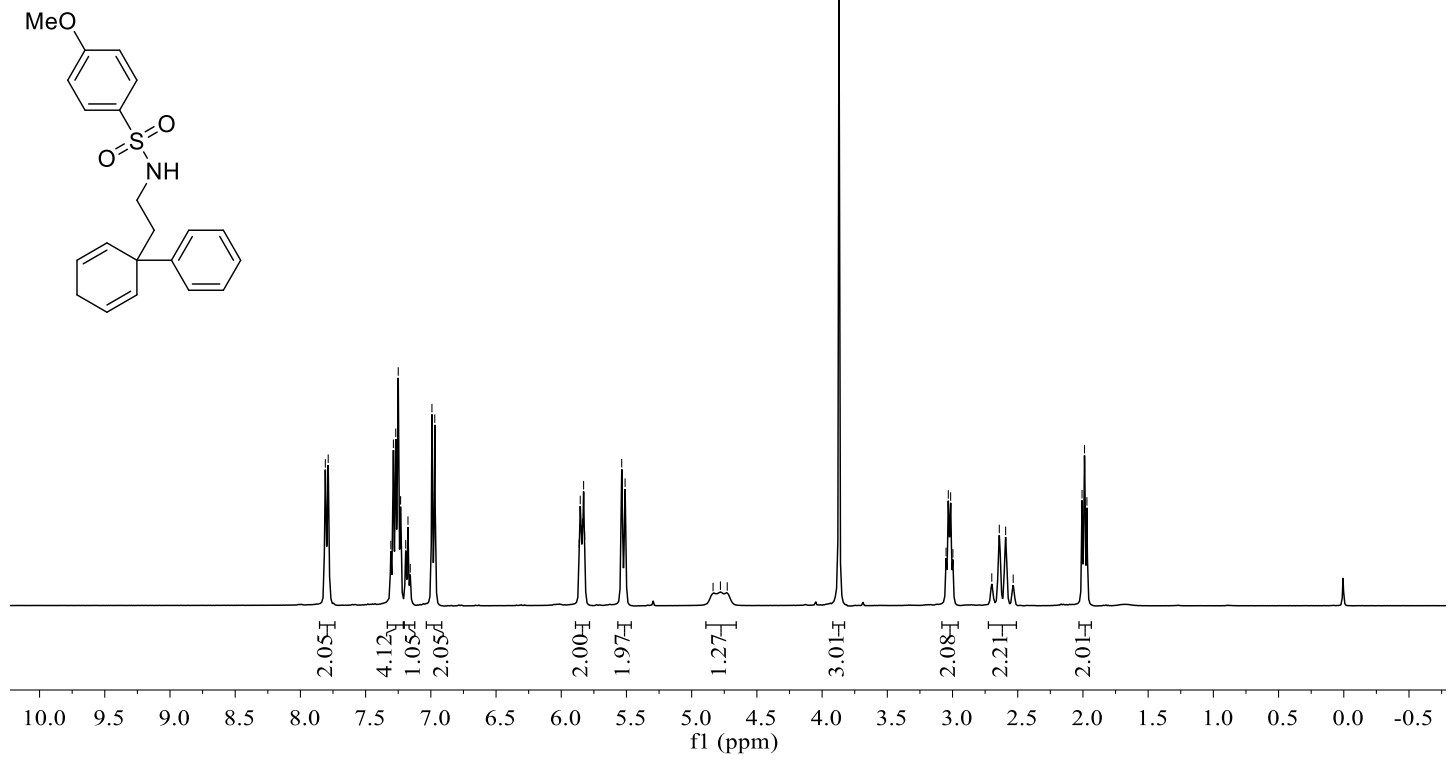

${ }^{13} \mathrm{C}$ NMR of $1 \mathrm{c}\left(100 \mathrm{MHz}, \mathrm{CDCl}_{3}\right)$

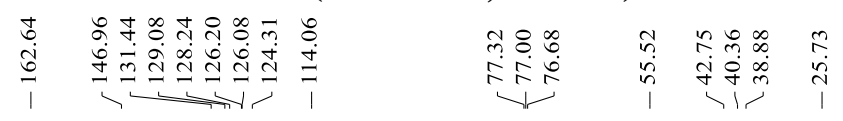
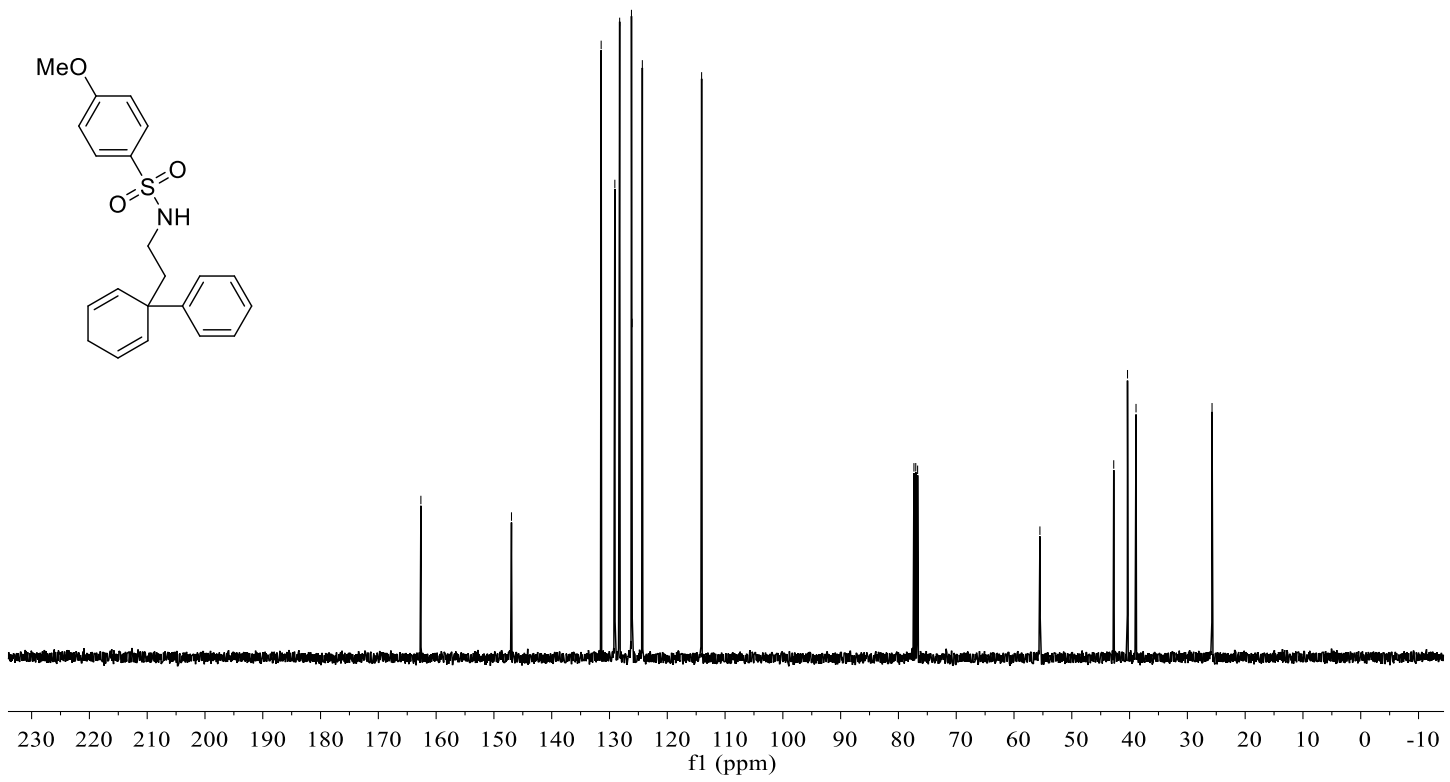

S31 
${ }^{1} \mathrm{H}$ NMR of $1 \mathrm{~d}\left(400 \mathrm{MHz}, \mathrm{CDCl}_{3}\right)$

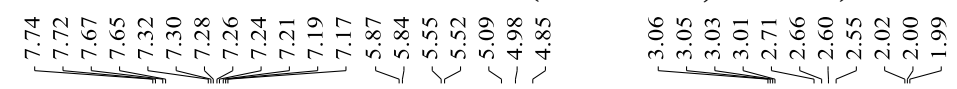
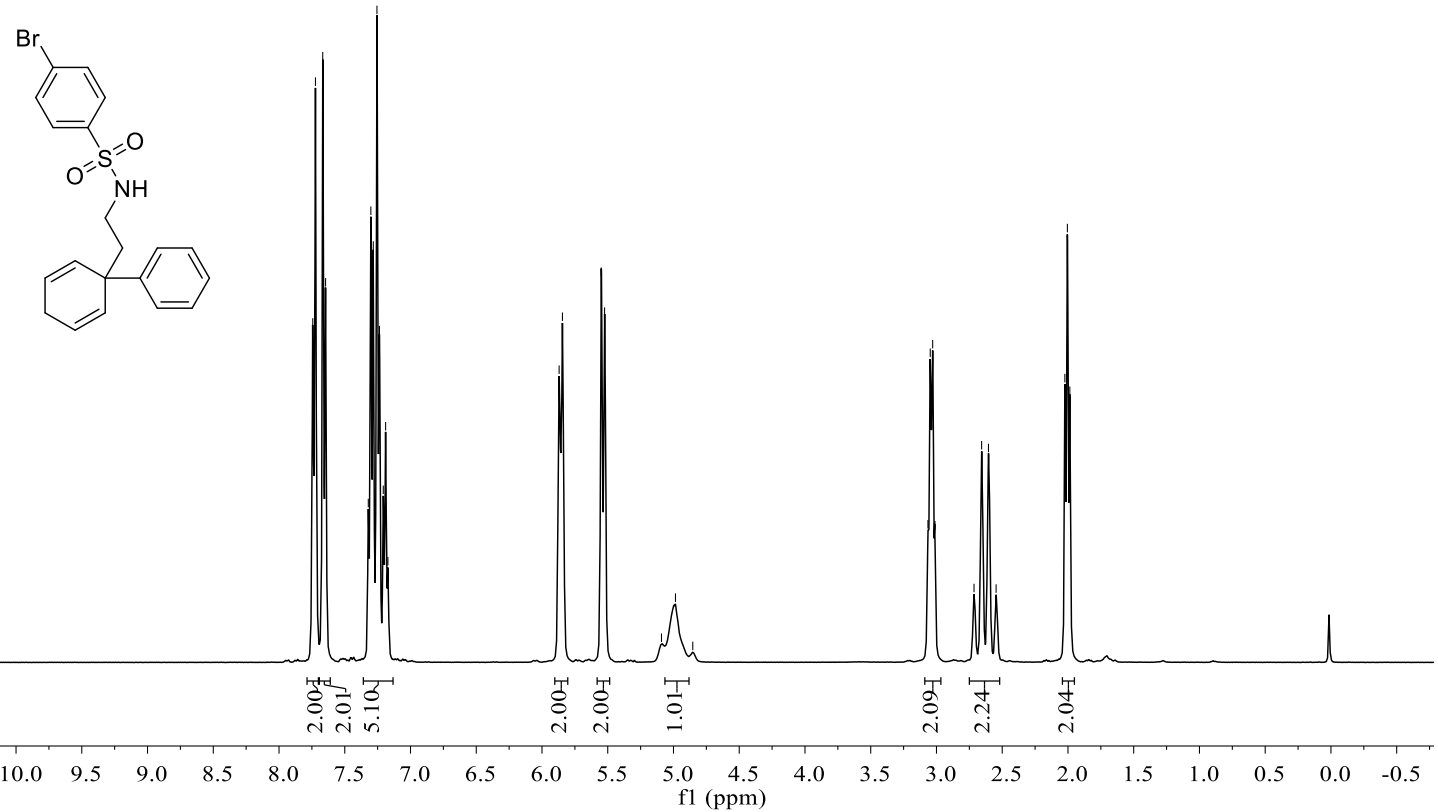

${ }^{13} \mathrm{C}$ NMR of $1 \mathrm{~d}\left(100 \mathrm{MHz}, \mathrm{CDCl}_{3}\right)$

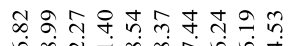

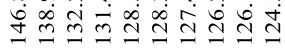

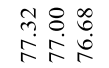

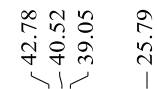

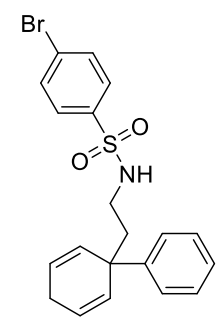

$\begin{array}{lllllllllllll}230 & 220 & 210 & 200 & 190 & 180 & 170 & 160 & 150 & 140 & 130 & 120 \begin{array}{l}110 \\ \mathrm{f} 1(\mathrm{ppm})\end{array}\end{array}$ 
${ }^{1} \mathrm{H}$ NMR of $1 \mathrm{e}\left(400 \mathrm{MHz}, \mathrm{CDCl}_{3}\right)$

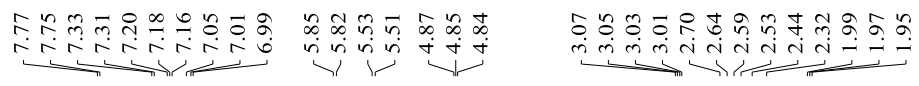

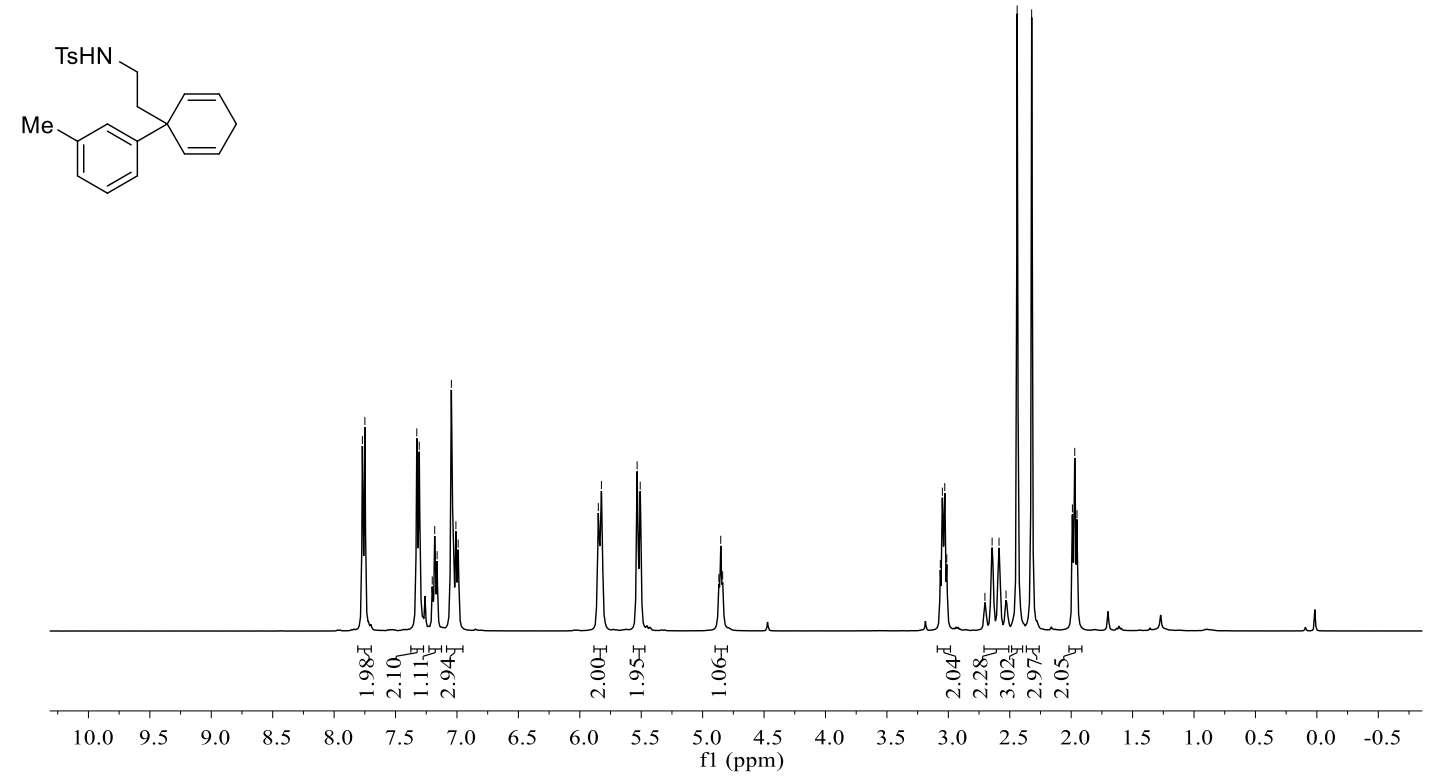

${ }^{13} \mathrm{C}$ NMR of $1 \mathrm{e}\left(100 \mathrm{MHz}, \mathrm{CDCl}_{3}\right)$

ㅇํำ 중ํำ

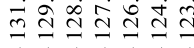

กำ

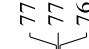

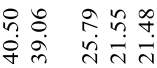

(SsHN

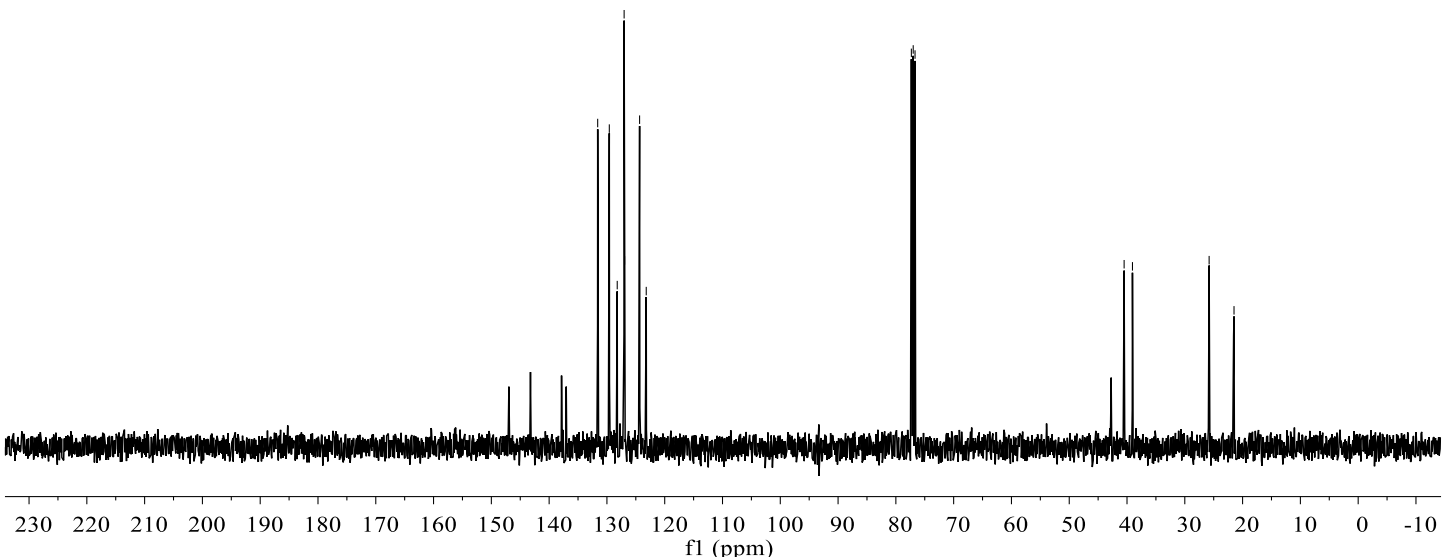




\section{${ }^{1} \mathrm{H}$ NMR of $1 f\left(400 \mathrm{MHz}, \mathrm{CDCl}_{3}\right)$}

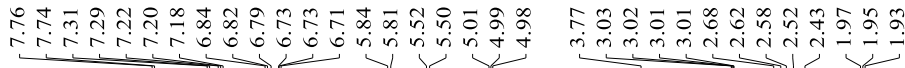

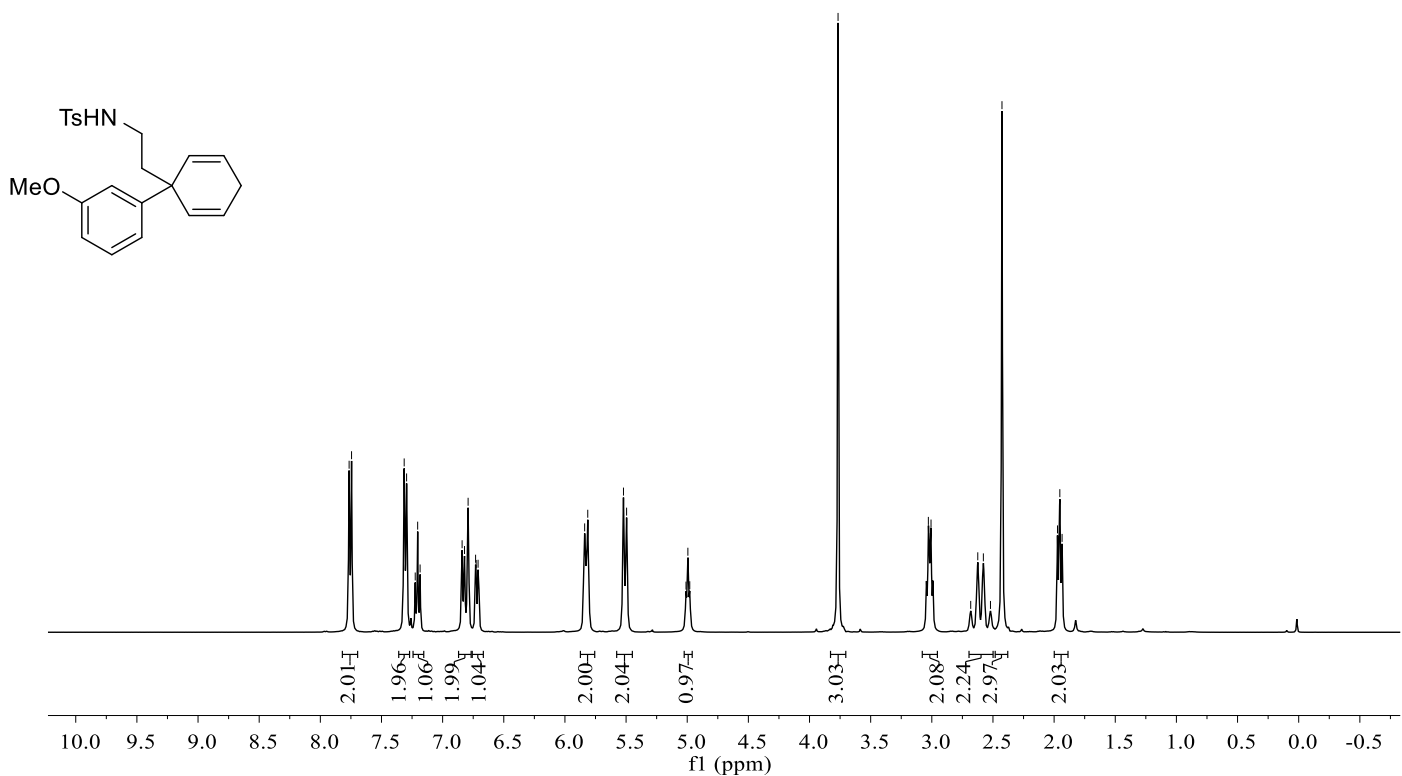

${ }^{13} \mathrm{C}$ NMR of $1 f\left(100 \mathrm{MHz}, \mathrm{CDCl}_{3}\right)$

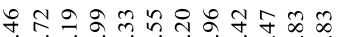

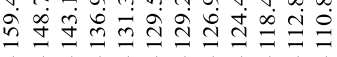

\section{용}

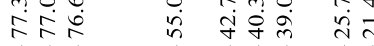
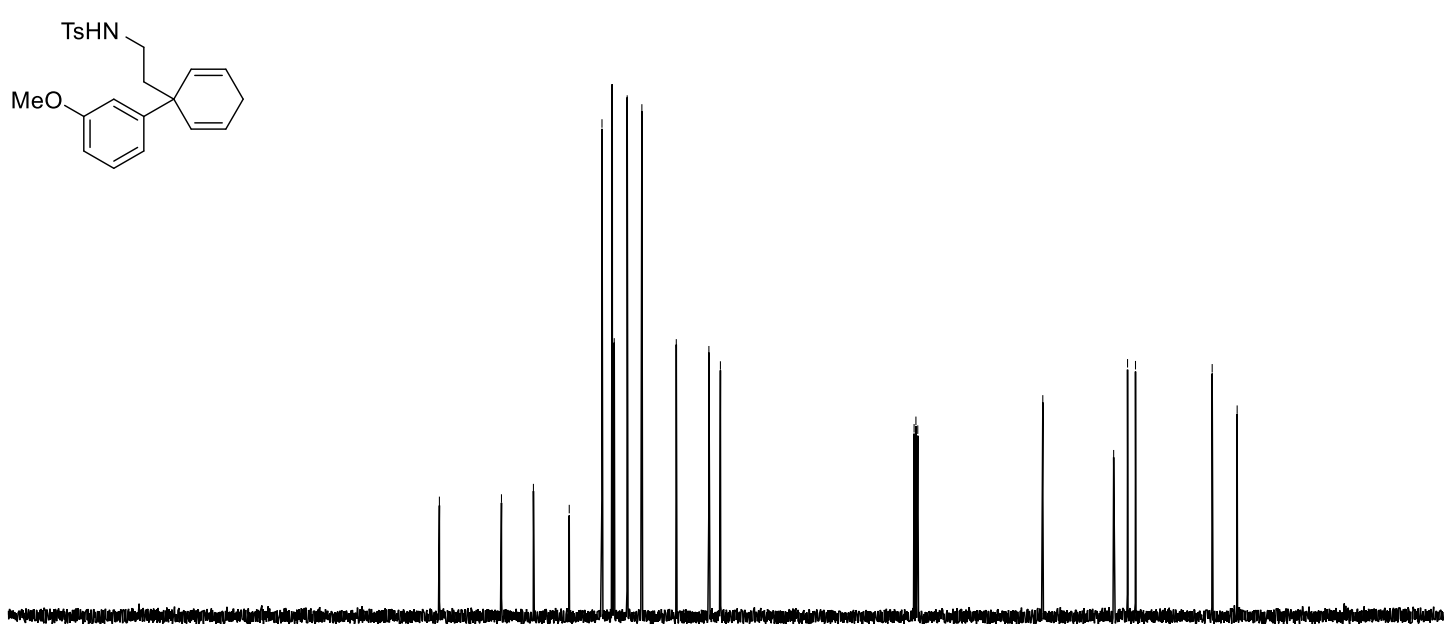

$\begin{array}{lllllllllllllllllllllllll}230 & 220 & 210 & 200 & 190 & 180 & 170 & 160 & 150 & 140 & 130 & 120 & 110 & 100 & 90 & 80 & 70 & 60 & 50 & 40 & 30 & 20 & 10 & 0 & -10\end{array}$ 


\section{${ }^{1} \mathrm{H}$ NMR of $1 \mathrm{~g}\left(400 \mathrm{MHz}, \mathrm{CDCl}_{3}\right)$}

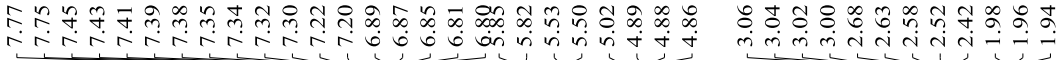<smiles>NCCc1cccc(O)c1</smiles>

${ }^{13} \mathrm{C}$ NMR of $1 \mathrm{~g}(100 \mathrm{MHz}, \mathrm{CDCl} 3)$

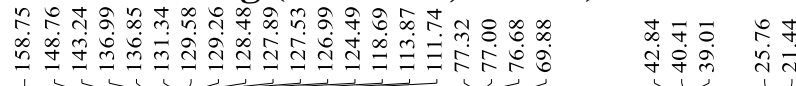

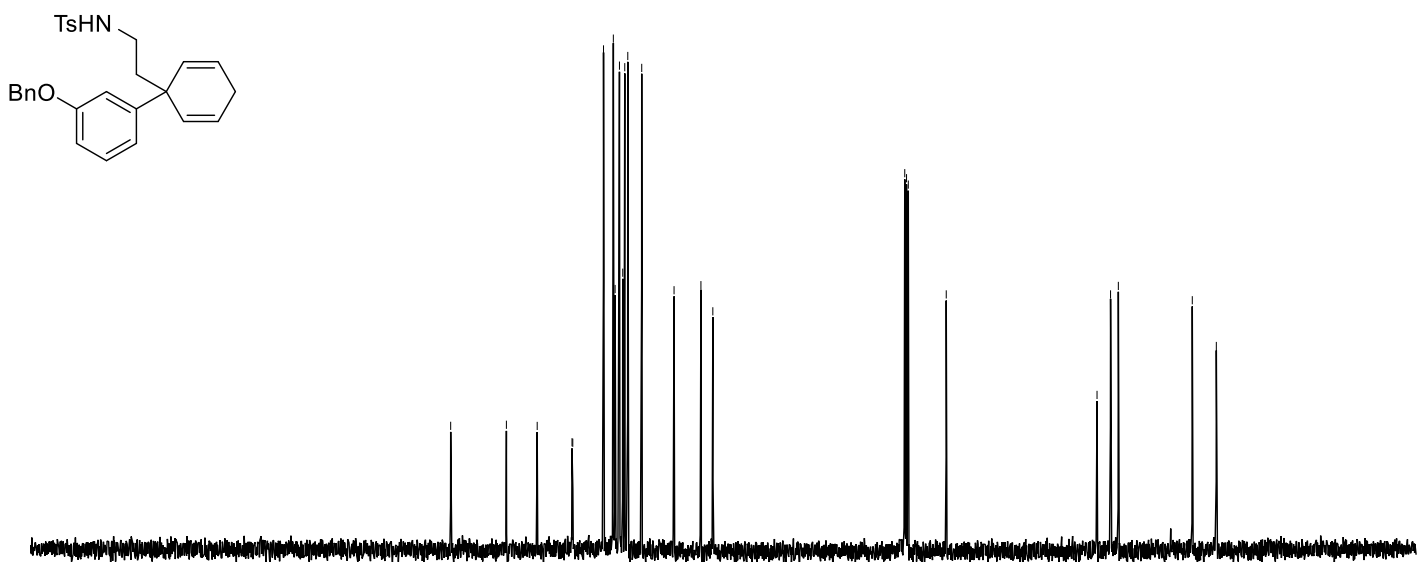

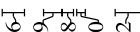

\begin{tabular}{|c|c|c|c|c|c|c|c|c|c|c|c|c|c|c|c|c|c|c|c|c|c|}
\hline & & & & & 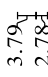 & 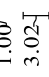 & & $\stackrel{T}{T}$ & 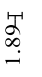 & 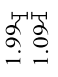 & & & & 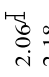 & $\begin{array}{l}\text { गुण } \\
\infty \\
\infty \\
i\end{array}$ & $\begin{array}{l}\frac{T}{T} \\
0 \\
i\end{array}$ & & & & & \\
\hline 10.0 & 9.5 & 9.0 & 8.5 & 8.0 & 7.5 & 7.0 & 6.5 & 6.0 & 5.5 & $\begin{array}{c}5.0 \\
\text { f1 }\end{array}$ & $\begin{array}{c}4.5 \\
\mathrm{pm})\end{array}$ & 4.0 & 3.5 & 3.0 & 2.5 & 2.0 & 1.5 & 1.0 & 0.5 & 0.0 & -0.5 \\
\hline
\end{tabular}

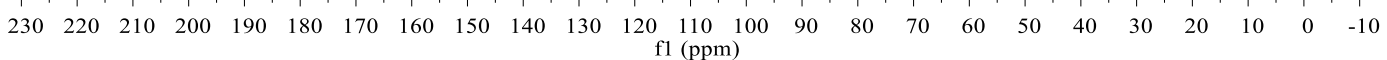


${ }^{1} \mathrm{H}$ NMR of $1 \mathrm{~h}\left(400 \mathrm{MHz}, \mathrm{CDCl}_{3}\right)$

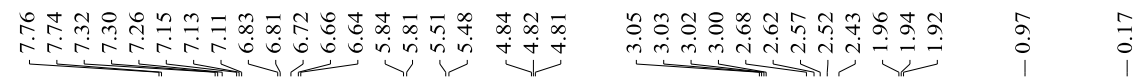

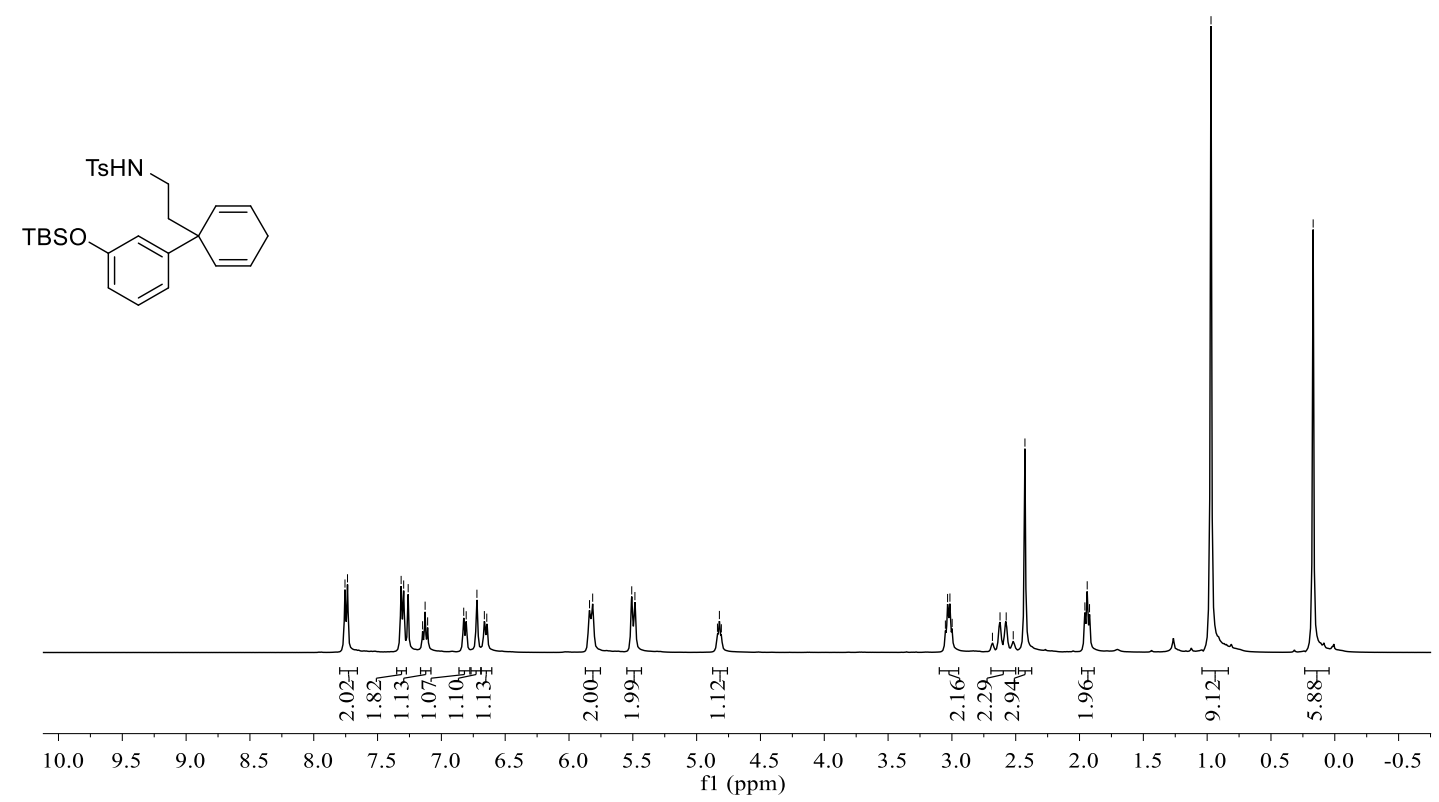

${ }^{13} \mathrm{C}$ NMR of ${ }^{1} \mathrm{H}$ NMR of $1 \mathrm{~h}\left(100 \mathrm{MHz}, \mathrm{CDCl}_{3}\right)$

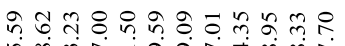

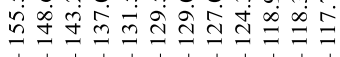

$\sqrt{n}$

茟员可

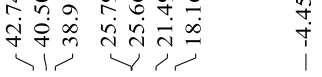
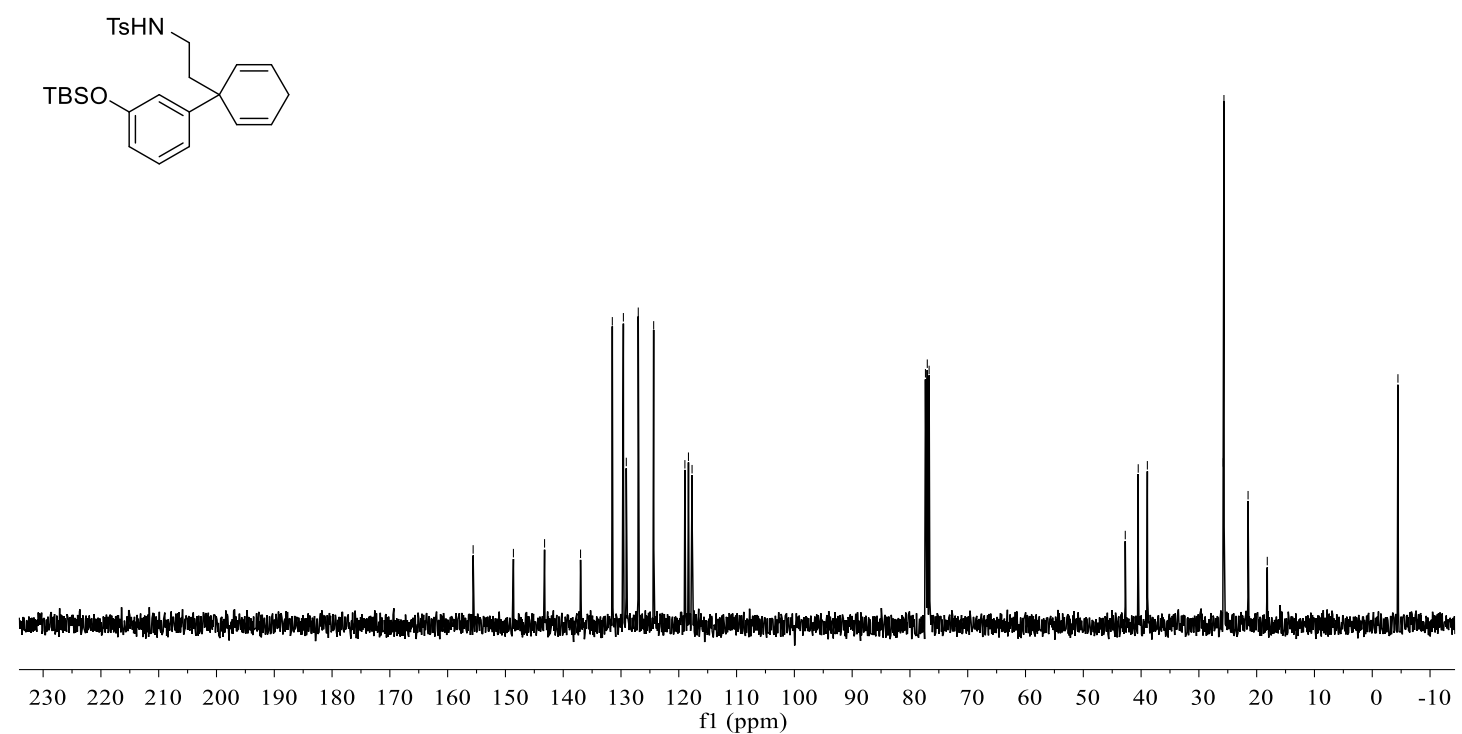
${ }^{1} \mathrm{H}$ NMR of ${ }^{1} \mathrm{H}$ NMR of $1 \mathrm{i}\left(400 \mathrm{MHz}, \mathrm{CDCl}_{3}\right)$<smiles>NCCC1(c2cccc(-c3ccccc3)c2)C=CCC=C1</smiles>

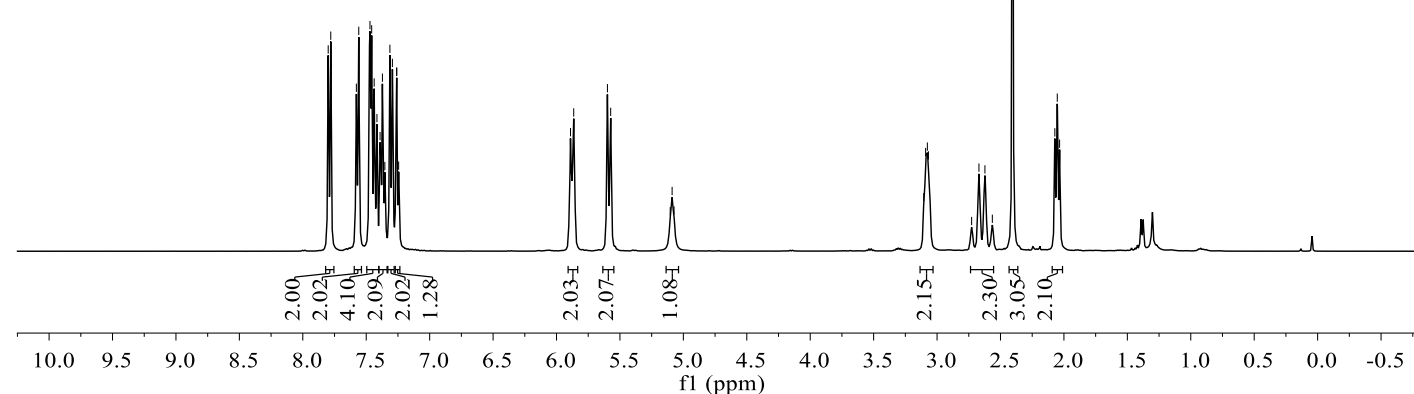

${ }^{13} \mathrm{C}$ NMR of $1 \mathrm{i}\left(100 \mathrm{MHz}, \mathrm{CDCl}_{3}\right)$

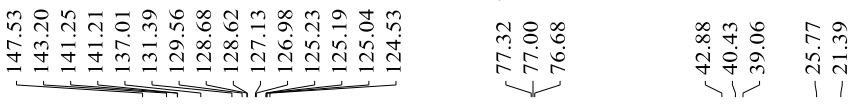<smiles>NCCC1(c2cccc(-c3ccccc3)c2)C=CCC=C1</smiles>

$\begin{array}{lllllllllllll}30 & 220 & 210 & 200 & 190 & 180 & 170 & 160 & 150 & 140 & 130 & 120 & 110 \quad 100 \\ \mathrm{f} 1(\mathrm{ppm})\end{array}$ 
${ }^{1} \mathrm{H}$ NMR of $1 \mathrm{j}\left(400 \mathrm{MHz}, \mathrm{CDCl}_{3}\right)$
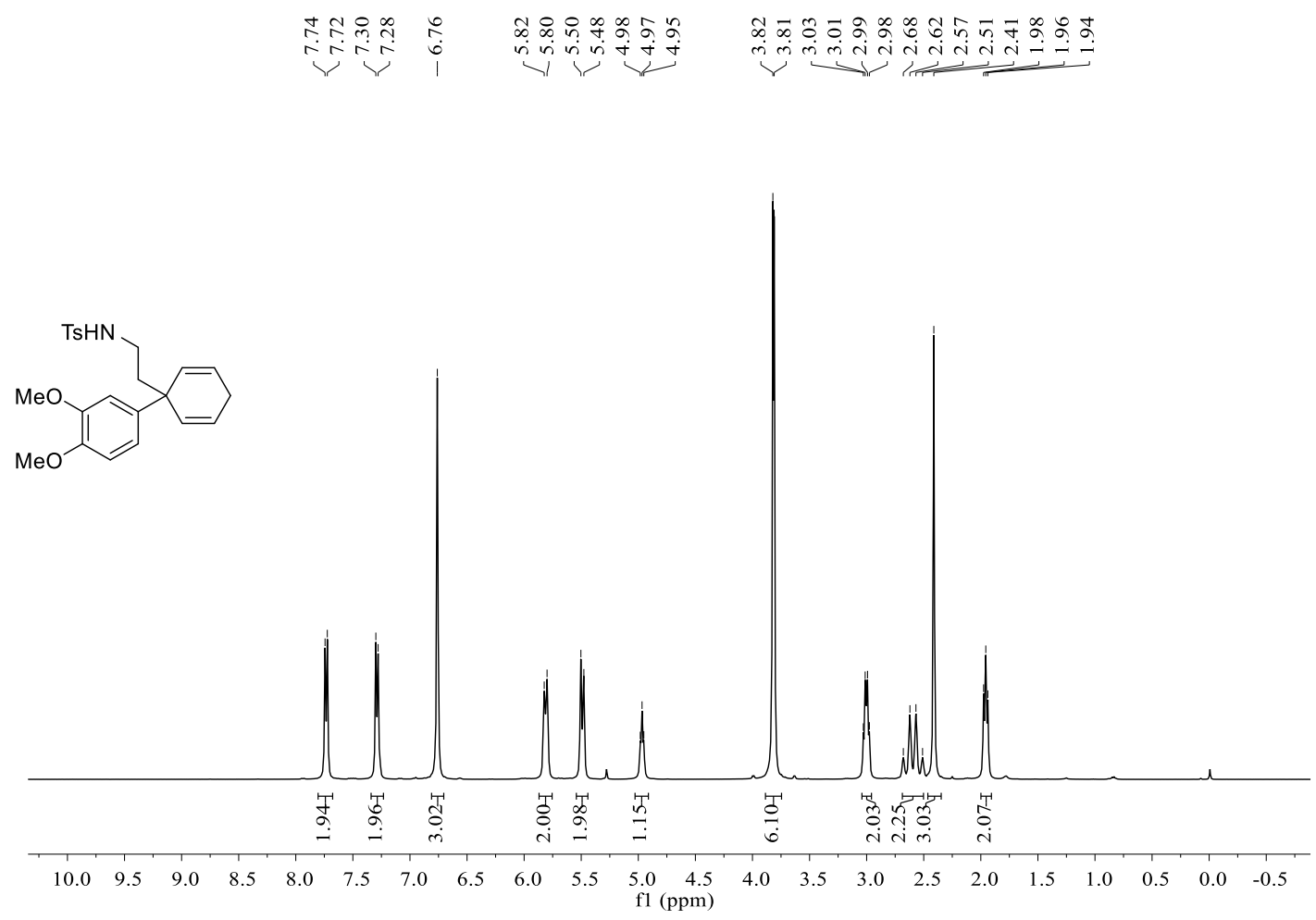

${ }^{13} \mathrm{C} \mathrm{NMR} \mathrm{of} 1 \mathrm{j}\left(100 \mathrm{MHz}, \mathrm{CDCl}_{3}\right)$

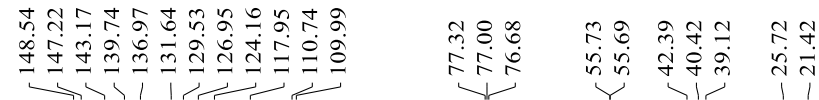
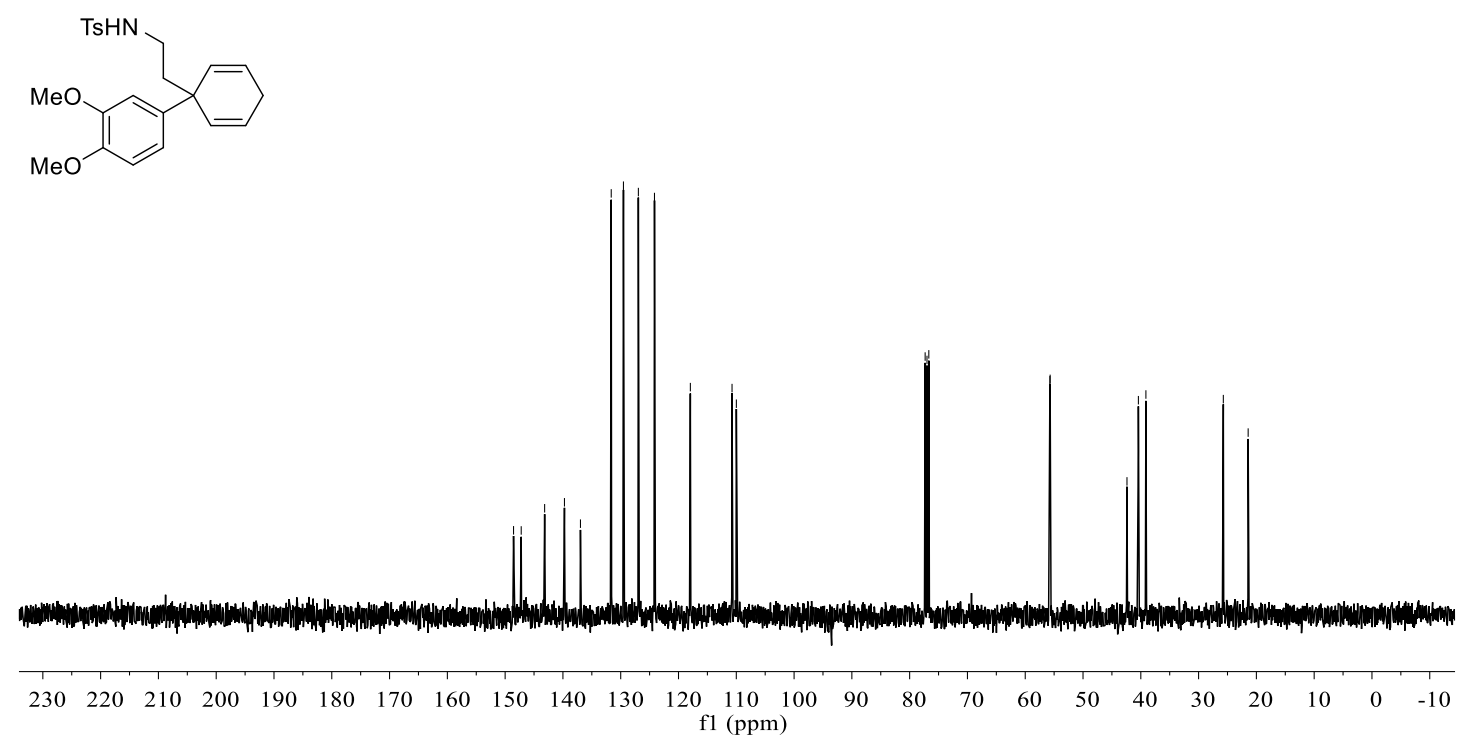
${ }^{1} \mathrm{H}$ NMR of $1 \mathrm{k}\left(400 \mathrm{MHz}, \mathrm{CDCl}_{3}\right)$

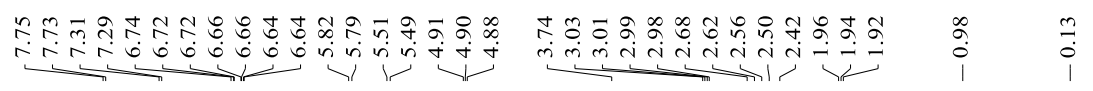<smiles>COc1ccc(C2(CCN[14CH3])C=CCC=C2)cc1OC</smiles>

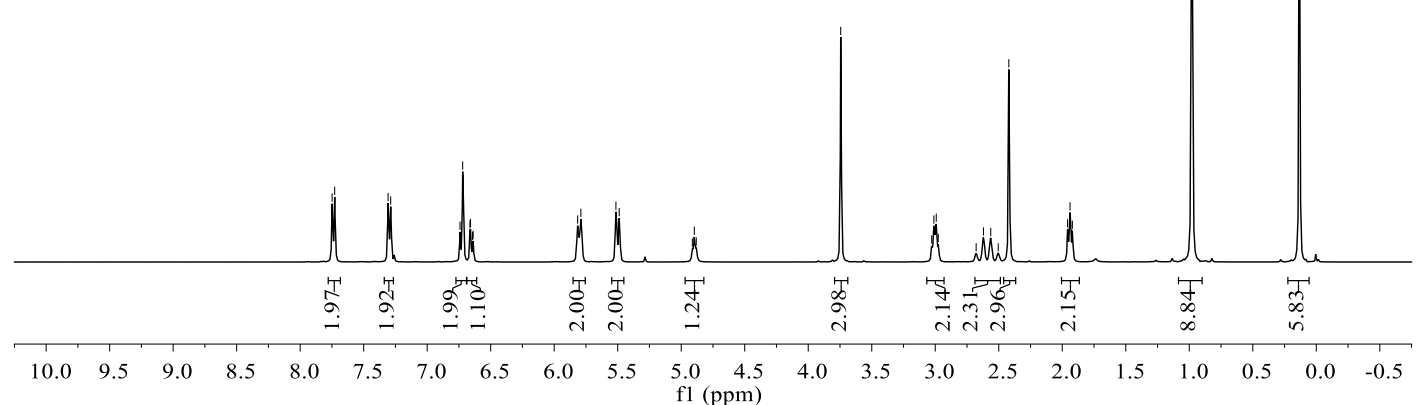

${ }^{13} \mathrm{C}$ NMR of $1 \mathrm{k}\left(100 \mathrm{MHz}, \mathrm{CDCl}_{3}\right)$

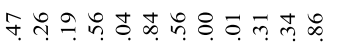

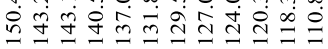

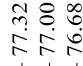

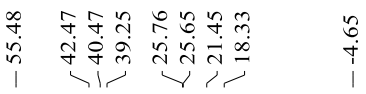
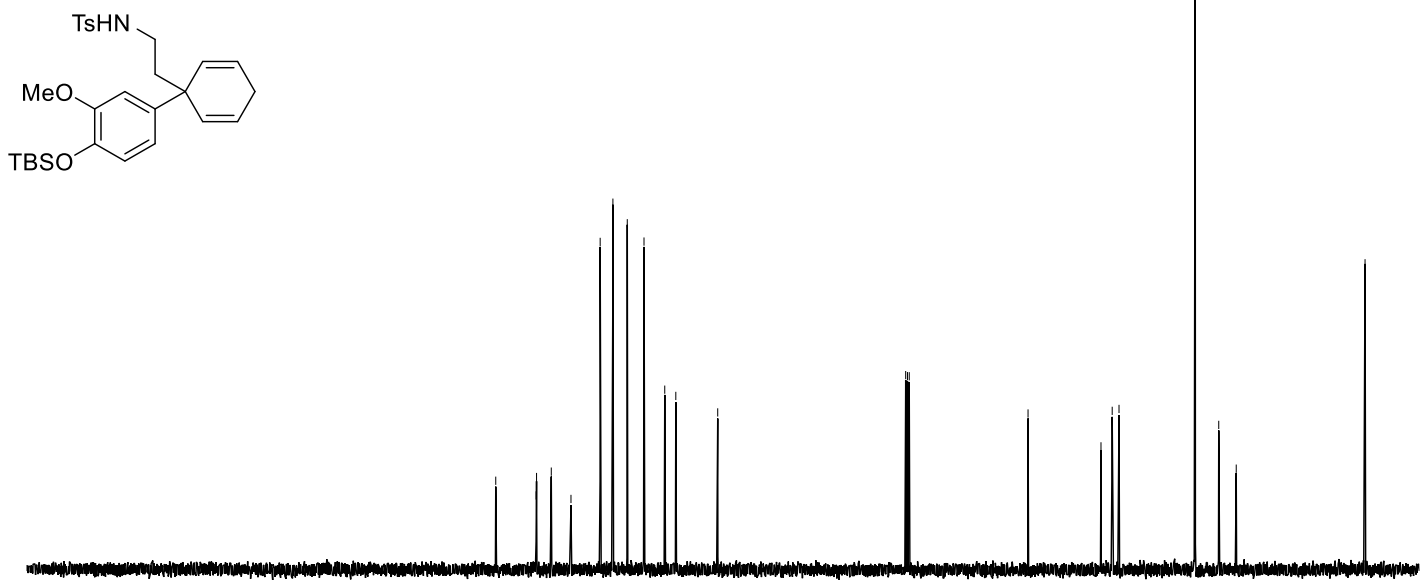

$\begin{array}{lllllllllllllllllllllllll}230 & 220 & 210 & 200 & 190 & 180 & 170 & 160 & 150 & 140 & 130 & 120 & 110 & 100 & 90 & 80 & 70 & 60 & 50 & 40 & 30 & 20 & 10 & 0 & -10\end{array}$ 
${ }^{1} \mathrm{H}$ NMR of 11 (400 MHz, CDCl3)

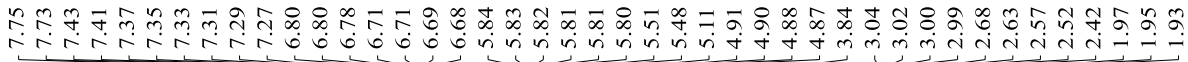<smiles>COc1ccc(C2(CCN)C=CCC2)cc1O</smiles>

${ }^{13} \mathrm{C}$ NMR of 11 (100 MHz, CDCl 3 )

茟

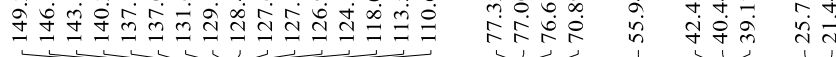

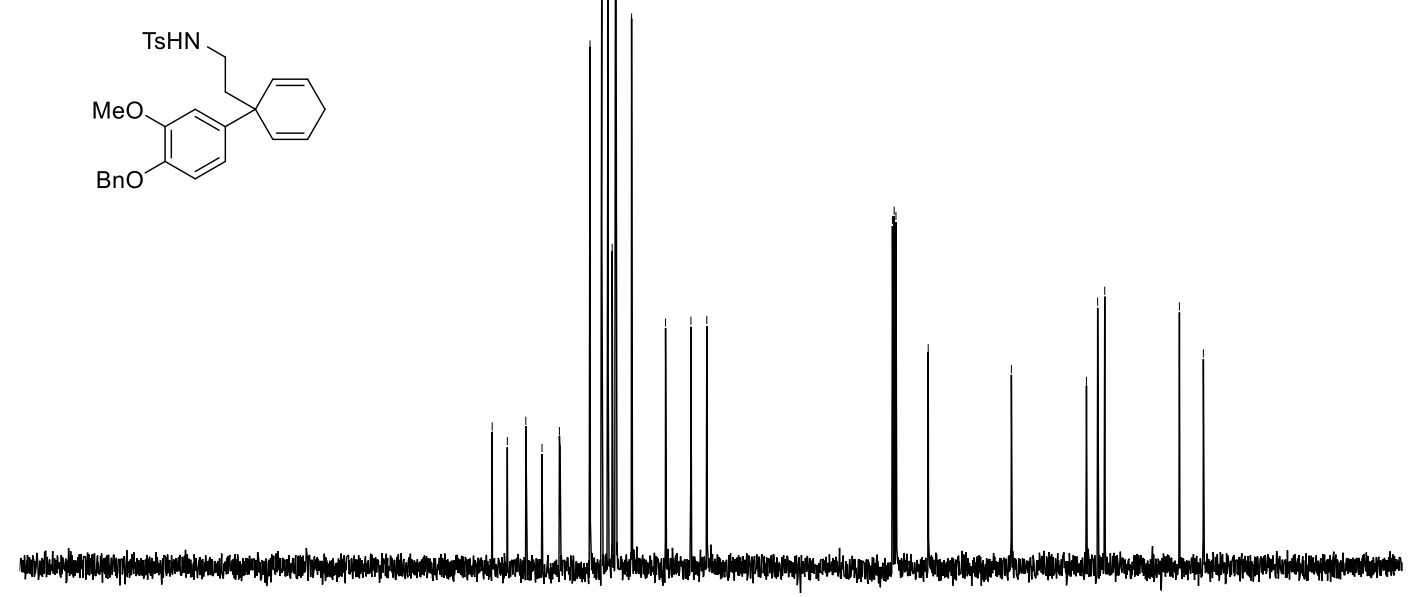

$\begin{array}{lllllllllllllllllllllllll}230 & 220 & 210 & 200 & 190 & 180 & 170 & 160 & 150 & 140 & 130 & 120 & 110 & 100 & 90 & 80 & 70 & 60 & 50 & 40 & 30 & 20 & 10 & 0 & -10\end{array}$ 
${ }^{1} \mathrm{H}$ NMR of $1 \mathrm{~m}\left(400 \mathrm{MHz}, \mathrm{CDCl}_{3}\right)$
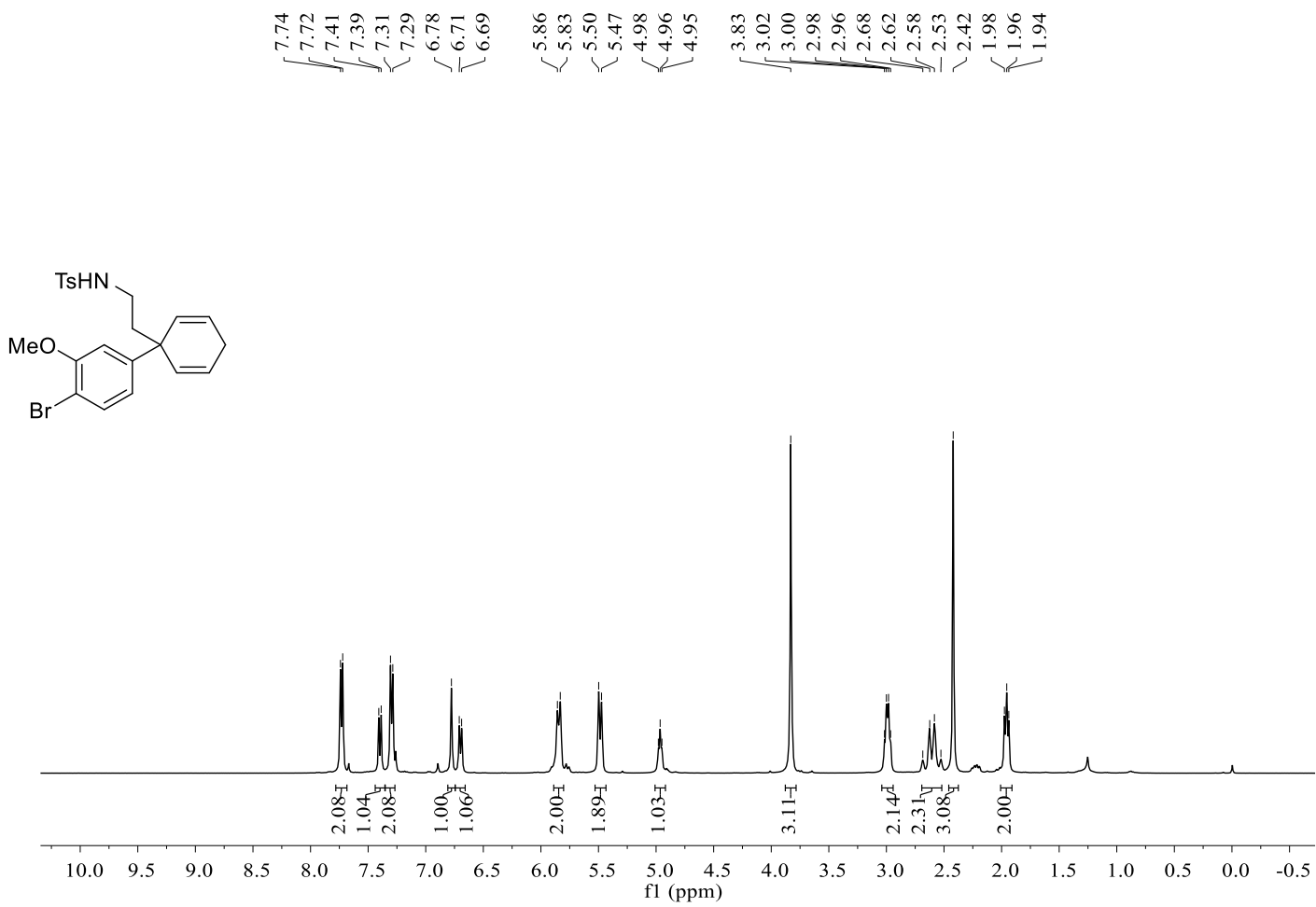

${ }^{13} \mathrm{C}$ NMR of $1 \mathrm{~m}\left(100 \mathrm{MHz}, \mathrm{CDCl}_{3}\right)$

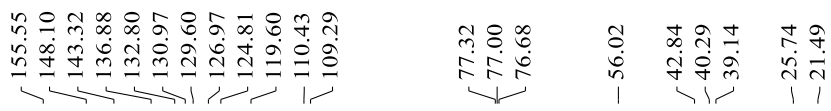<smiles>COc1cc(C2(CCN)C=CCCC2)ccc1Br</smiles>

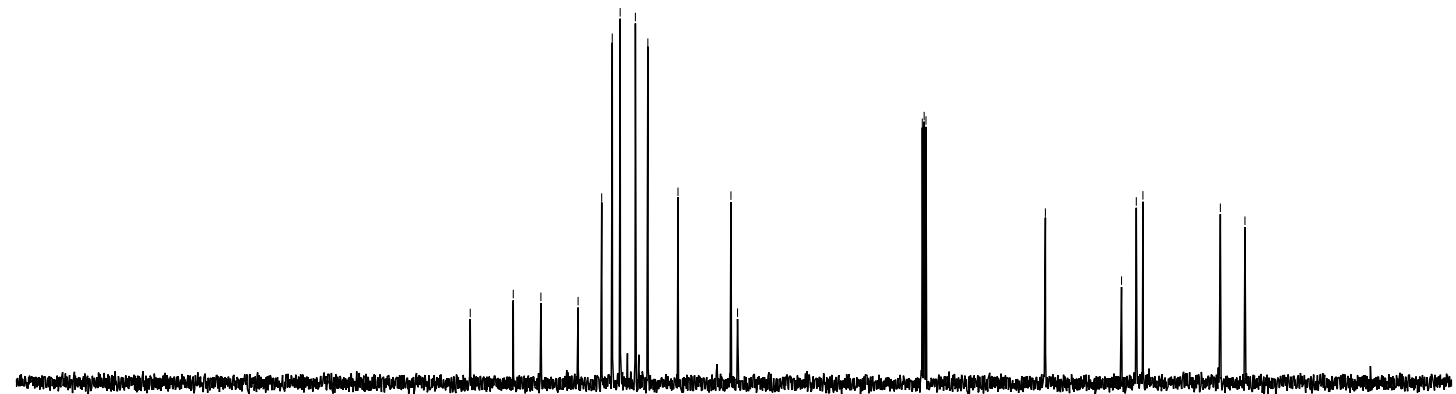

$\begin{array}{lllllllllllllllllllllllll}230 & 220 & 210 & 200 & 190 & 180 & 170 & 160 & 150 & 140 & 130 & 120 & 110 & 100 & 90 & 80 & 70 & 60 & 50 & 40 & 30 & 20 & 10 & 0 & -10\end{array}$ 
${ }^{1} \mathrm{H}$ NMR of 1n (400 MHz, $\left.\mathrm{CDCl}_{3}\right)$

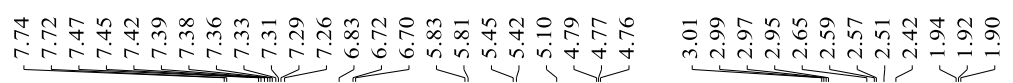

(TSHN)

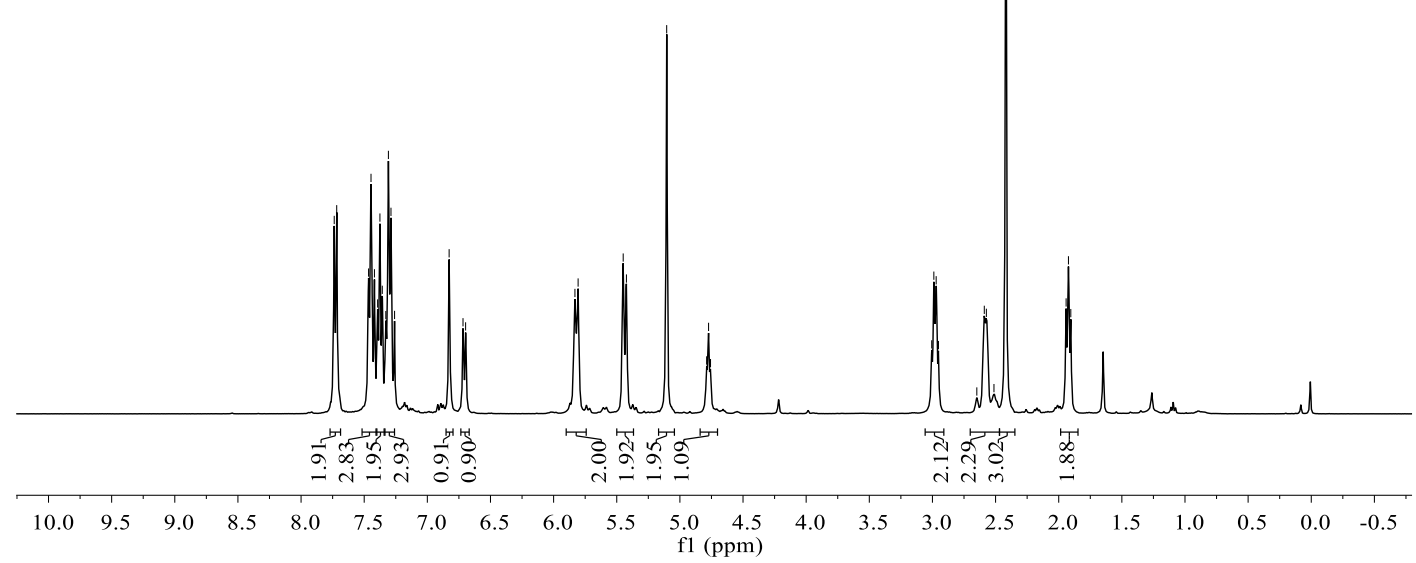

${ }^{13} \mathrm{C}$ NMR of $1 \mathrm{n}\left(100 \mathrm{MHz}, \mathrm{CDCl}_{3}\right)$

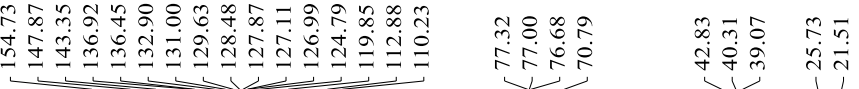

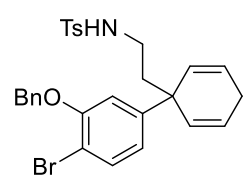

$\begin{array}{rrrrrrrrrr}200 & 190 & 180 & 170 & 160 & 150 & 140 & 130 & 120 & 110 \\ \text { f1 (ppm) }\end{array}$ 
${ }^{1} \mathrm{H}$ NMR of $\left.10\left(400 \mathrm{MHz}^{\mathrm{CDCl}}\right)_{3}\right)$

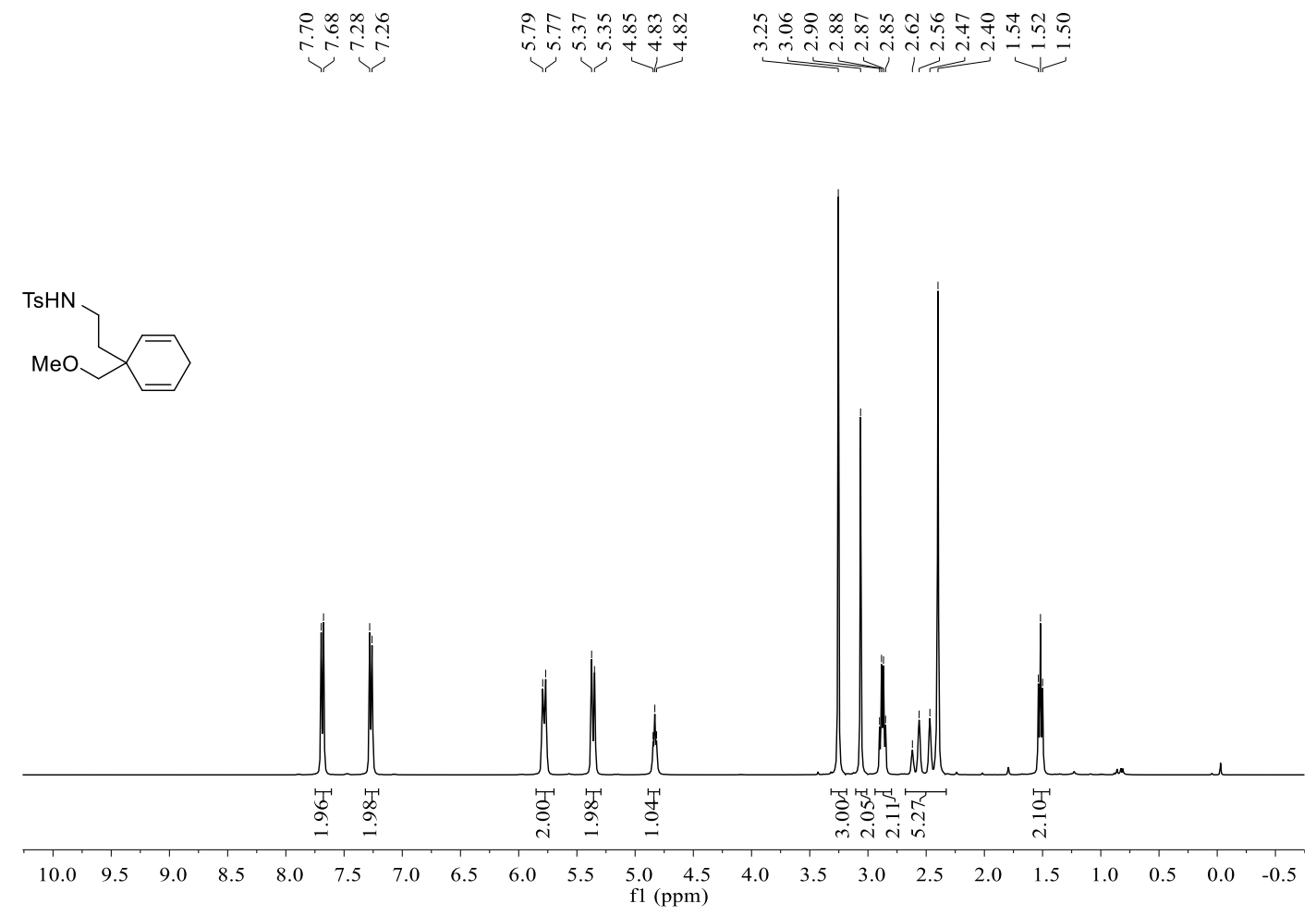

${ }^{13} \mathrm{C}$ NMR of $10\left(100 \mathrm{MHz}, \mathrm{CDCl}_{3}\right)$

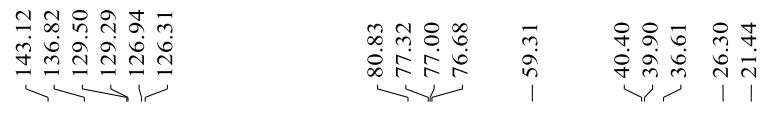

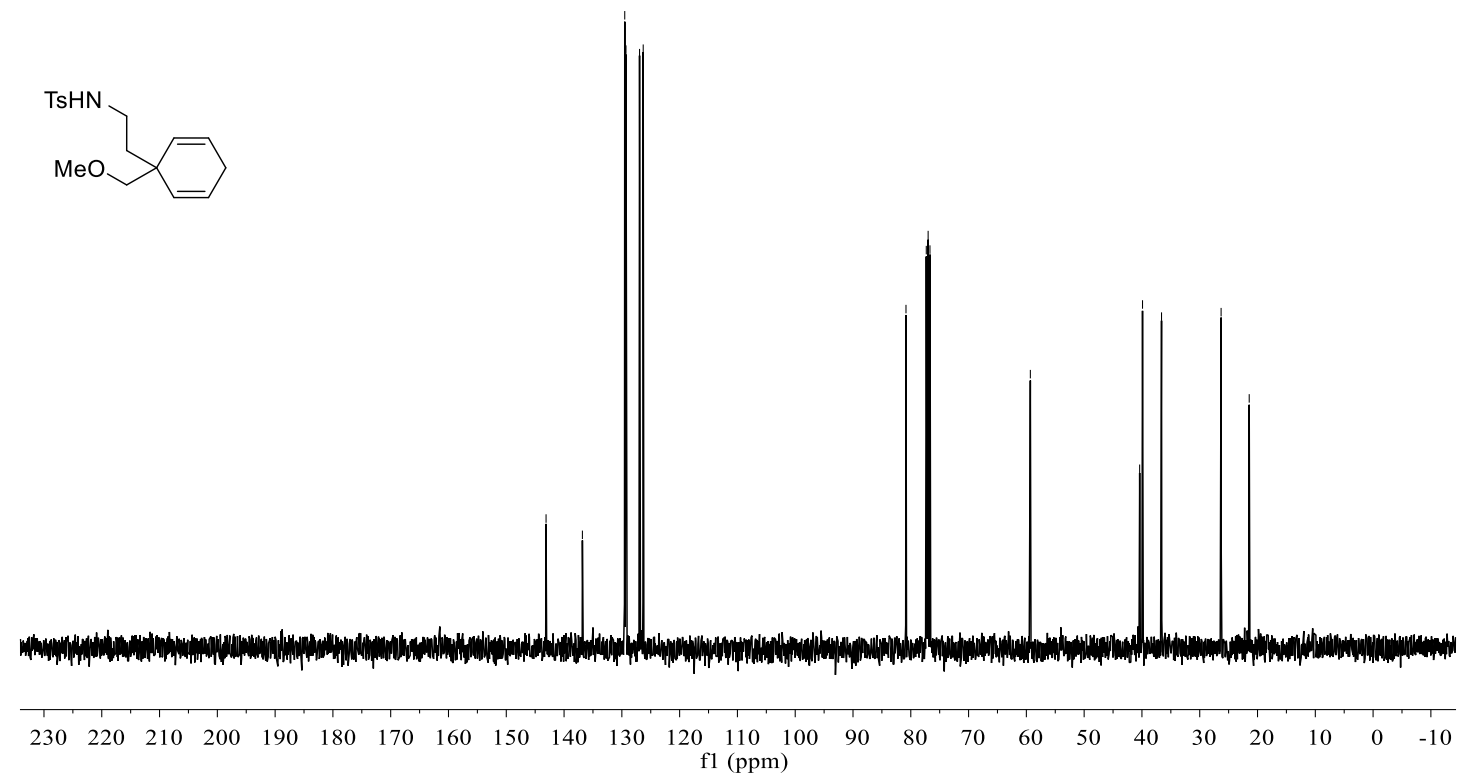


${ }^{1} \mathrm{H}$ NMR of 1p (400 MHz, $\left.\mathrm{CDCl}_{3}\right)$

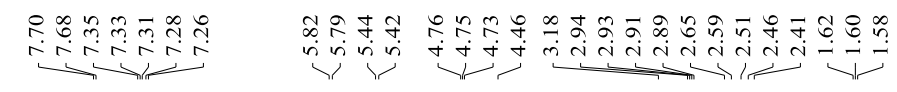

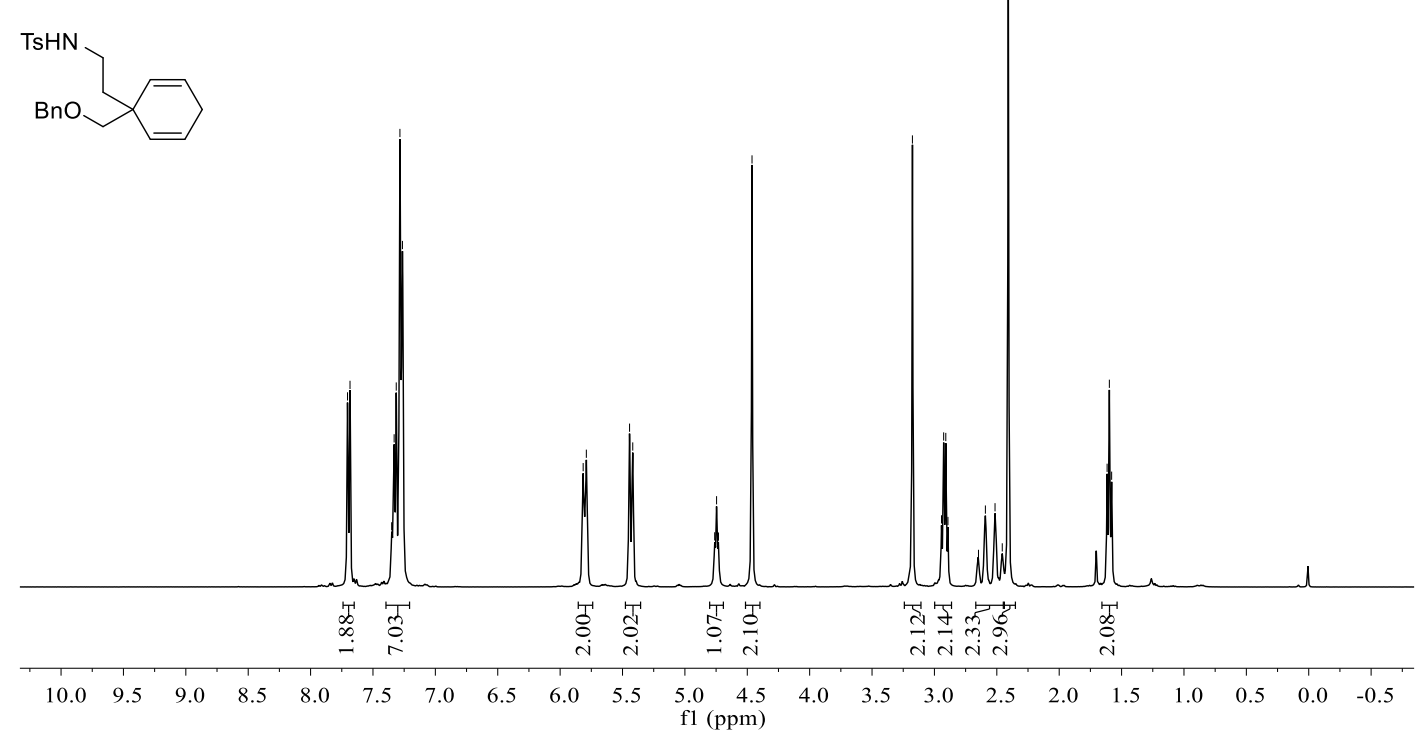

${ }^{13} \mathrm{C}$ NMR of 1p (100 $\left.\mathrm{MHz}, \mathrm{CDCl}_{3}\right)$

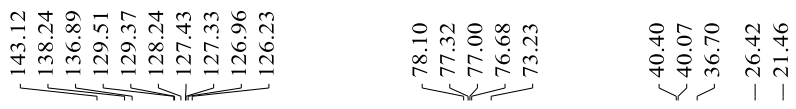



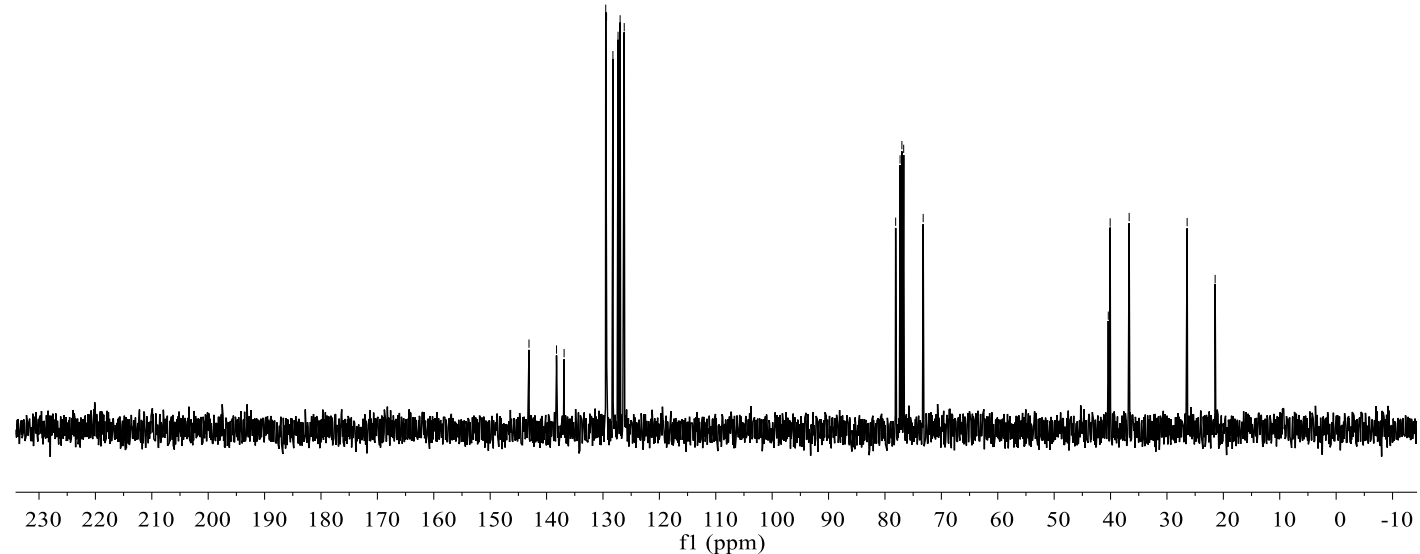


${ }^{1} \mathrm{H}$ NMR of $1 q\left(400 \mathrm{MHz}, \mathrm{CDCl}_{3}\right)$

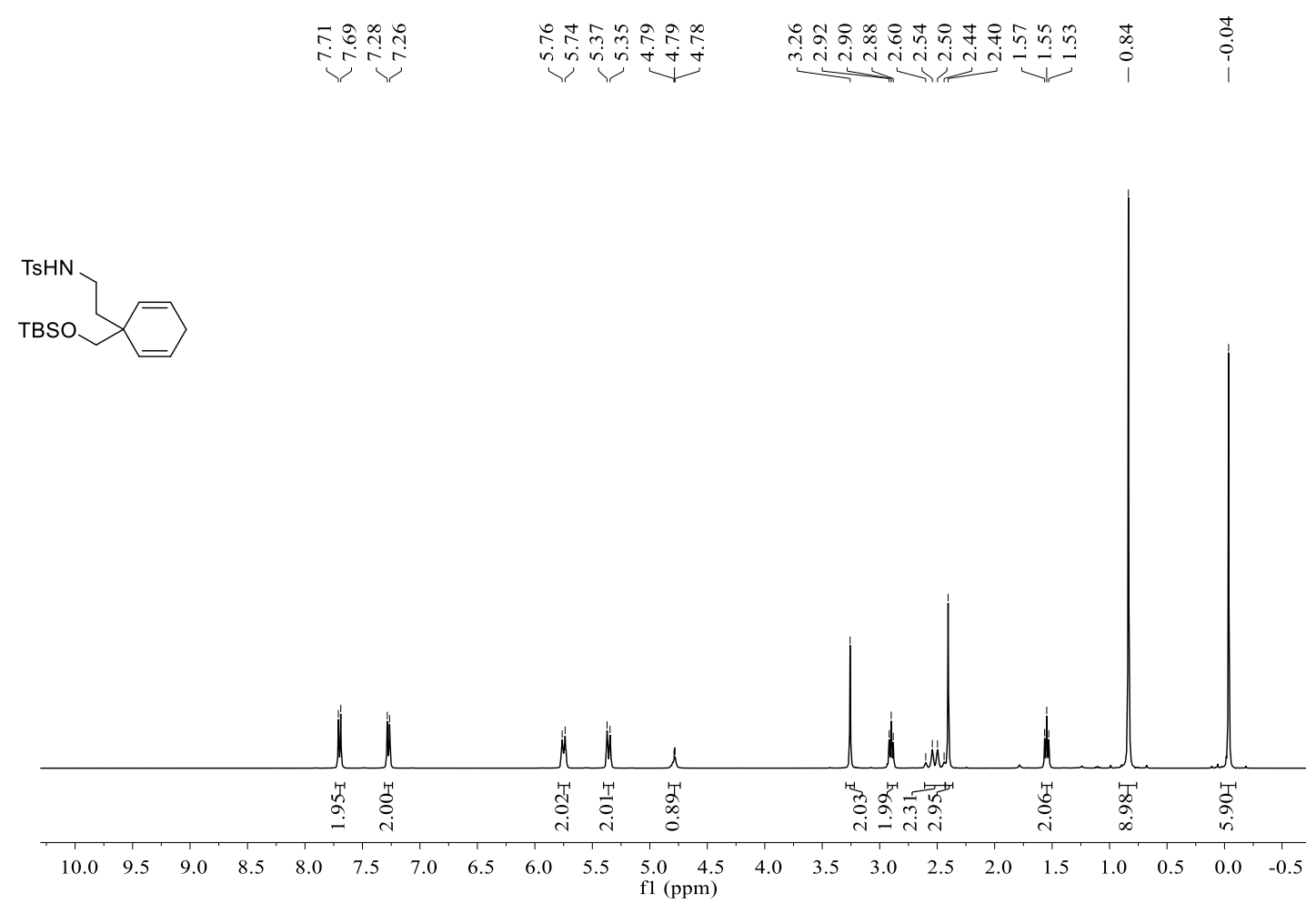

${ }^{13} \mathrm{C}$ NMR of $1 \mathrm{q}\left(100 \mathrm{MHz}, \mathrm{CDCl}_{3}\right)$

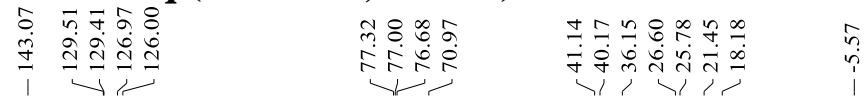

${ }^{\mathrm{TSHN}} \underset{-}{\mathrm{TBSO}}$

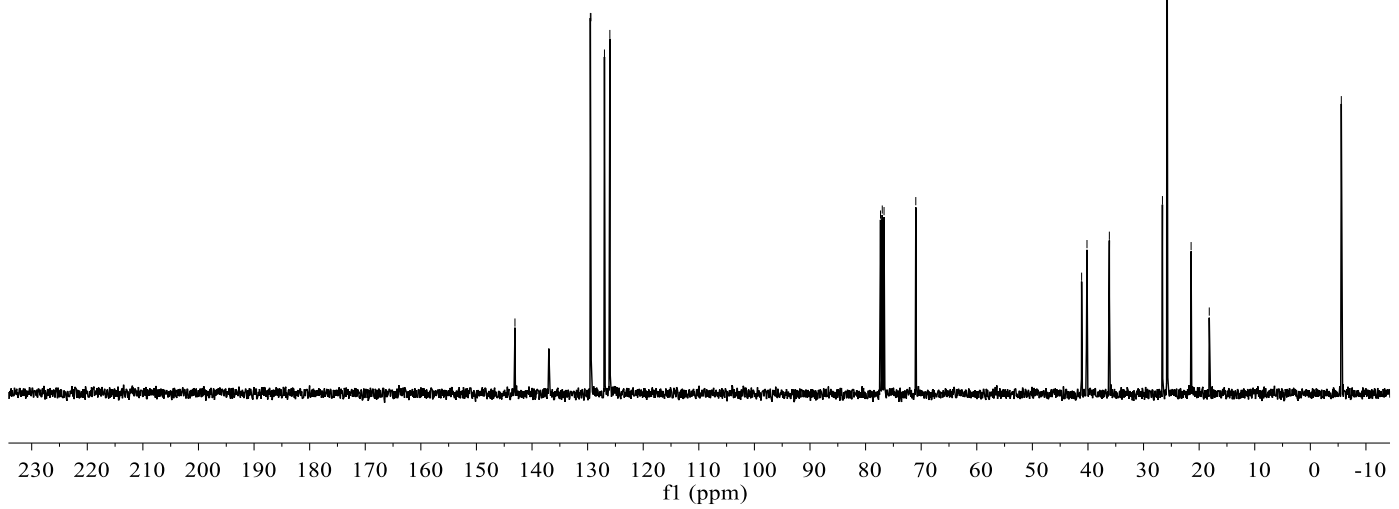


${ }^{1} \mathrm{H}$ NMR of $1 \mathrm{r}\left(400 \mathrm{MHz}, \mathrm{CDCl}_{3}\right)$

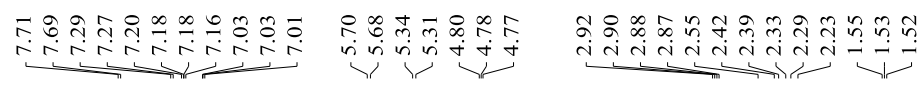

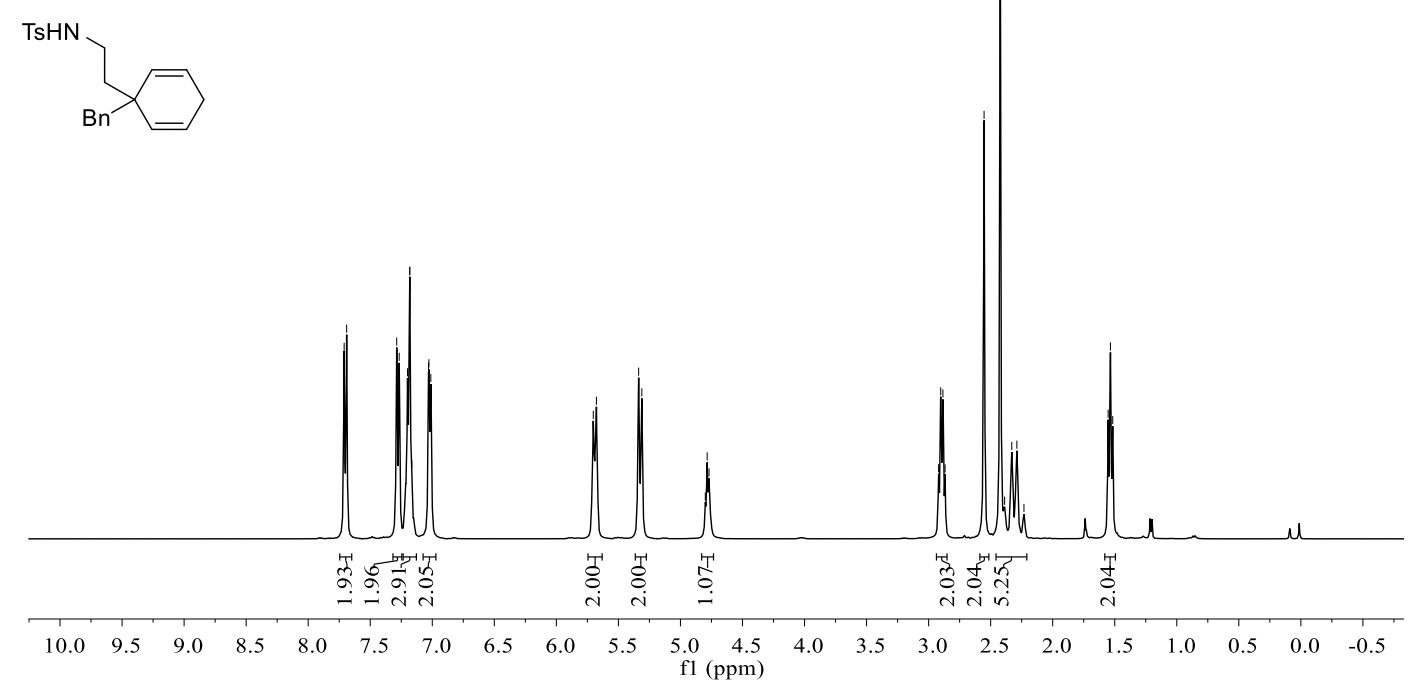

${ }^{13} \mathrm{C}$ NMR of $1 \mathrm{r}\left(100 \mathrm{MHz}, \mathrm{CDCl}_{3}\right)$

웅 ㅇํㅇ

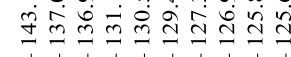

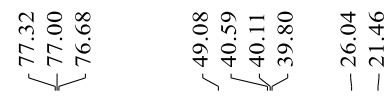

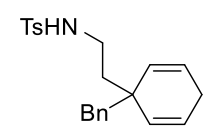

$\begin{array}{lllllllllllllllllllllllll}230 & 220 & 210 & 200 & 190 & 180 & 170 & 160 & 150 & 140 & 130 & 120 & 110 & 100 & 90 & 80 & 70 & 60 & 50 & 40 & 30 & 20 & 10 & 0 & -10\end{array}$ 
${ }^{1} \mathrm{H}$ NMR of $1 \mathrm{~s}\left(400 \mathrm{MHz}, \mathrm{CDCl}_{3}\right)$

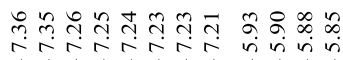

îd

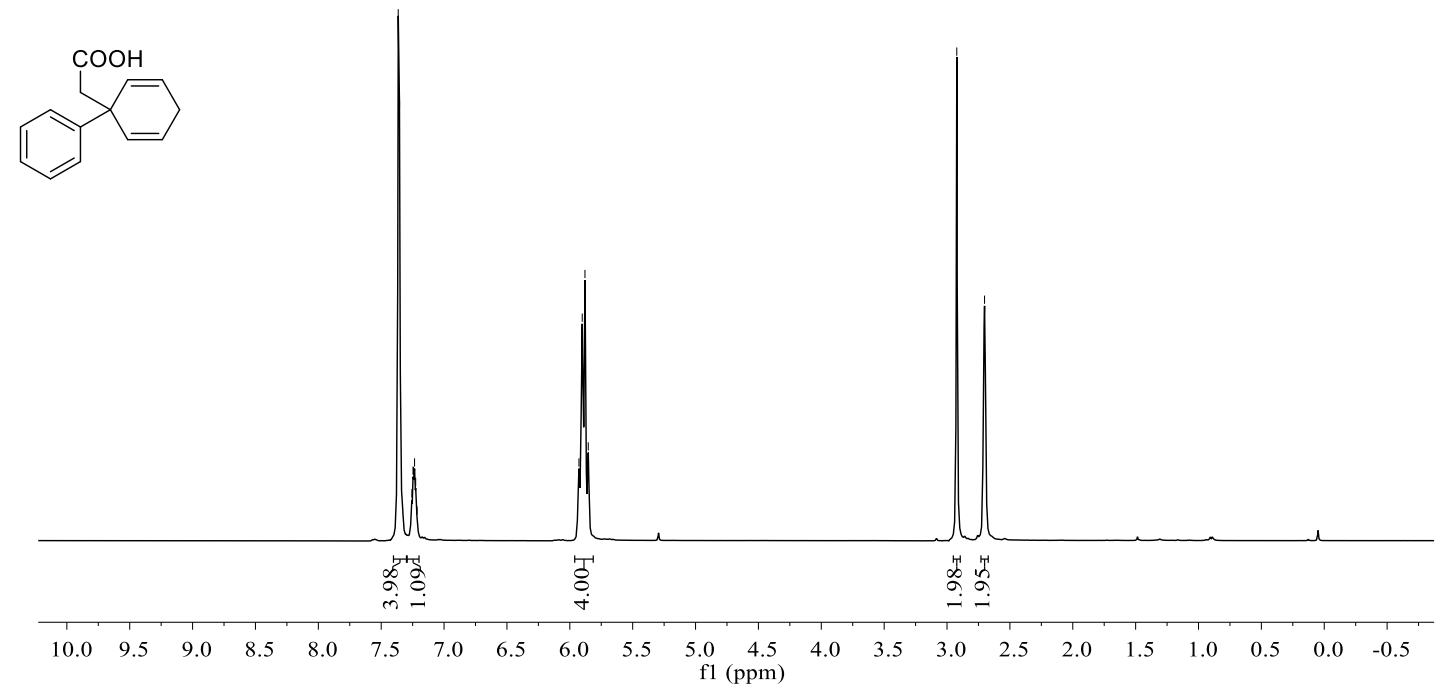

${ }^{13} \mathrm{C}$ NMR of $1 \mathrm{~s}\left(100 \mathrm{MHz}, \mathrm{CDCl}_{3}\right)$

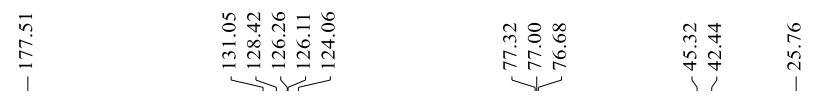<smiles>O=C(O)CC(C(=O)O)(c1ccccc1)c1ccccc1</smiles>

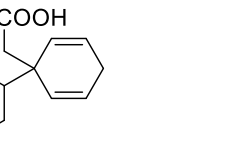

$\begin{array}{llllllllllllll}230 & 220 & 210 & 200 & 190 & 180 & 170 & 160 & 150 & 140 & 130 & 120 & 110 & 100\end{array}$ 
${ }^{1} \mathrm{H}$ NMR of $2 \mathrm{a}\left(400 \mathrm{MHz}, \mathrm{CDCl}_{3}\right)$

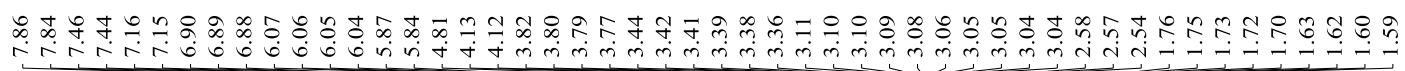

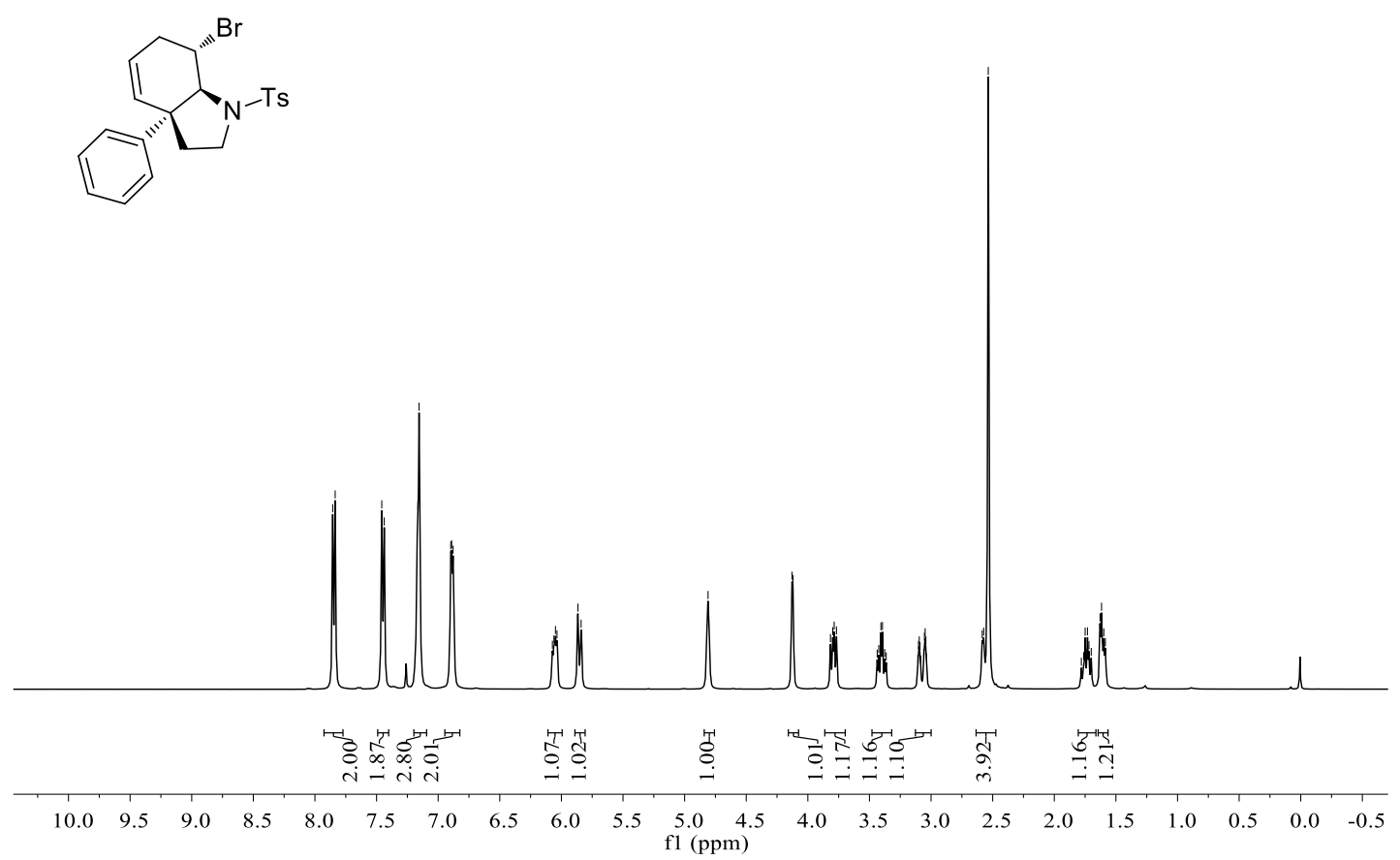

${ }^{13} \mathrm{C}$ NMR of 2a (100 MHz, $\left.\mathrm{CDCl}_{3}\right)$

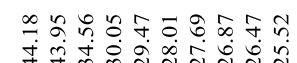

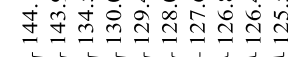

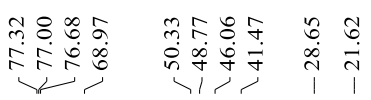

Br

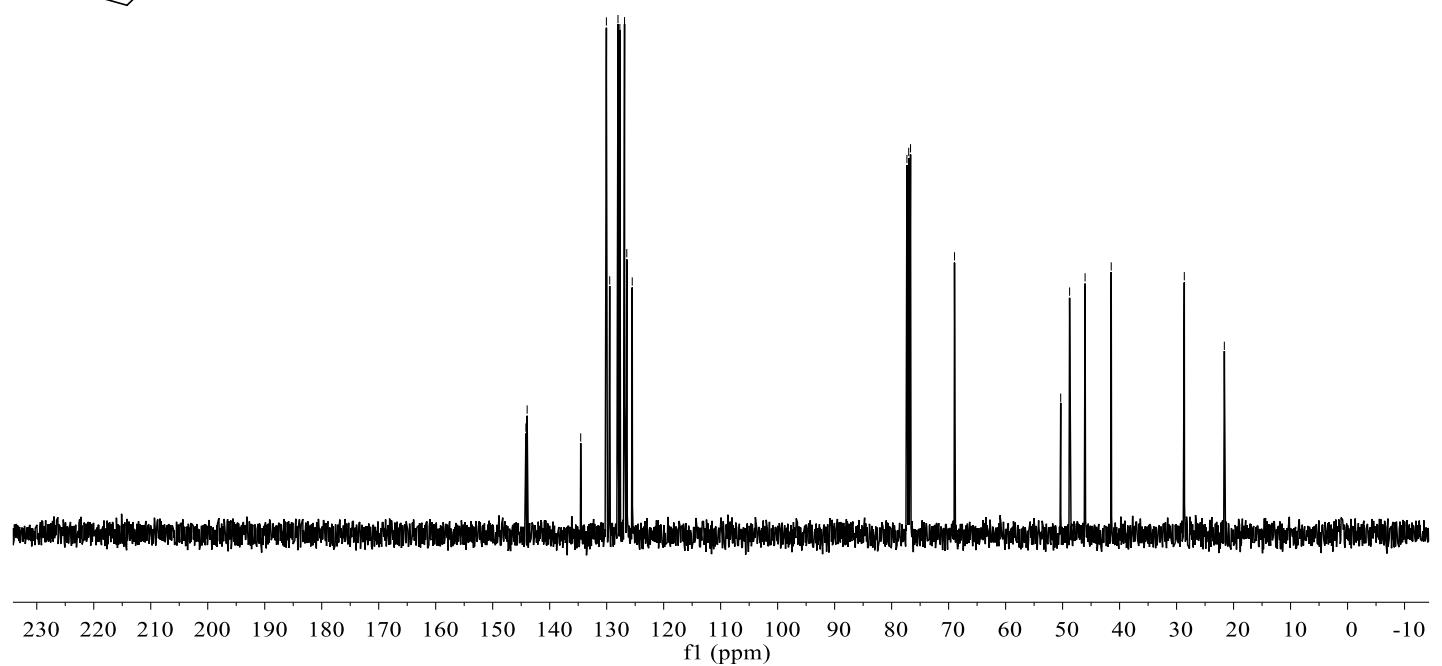


${ }^{1} \mathrm{H}$ NMR of 2a' (400 $\left.\mathrm{MHz}, \mathrm{CDCl}_{3}\right)$

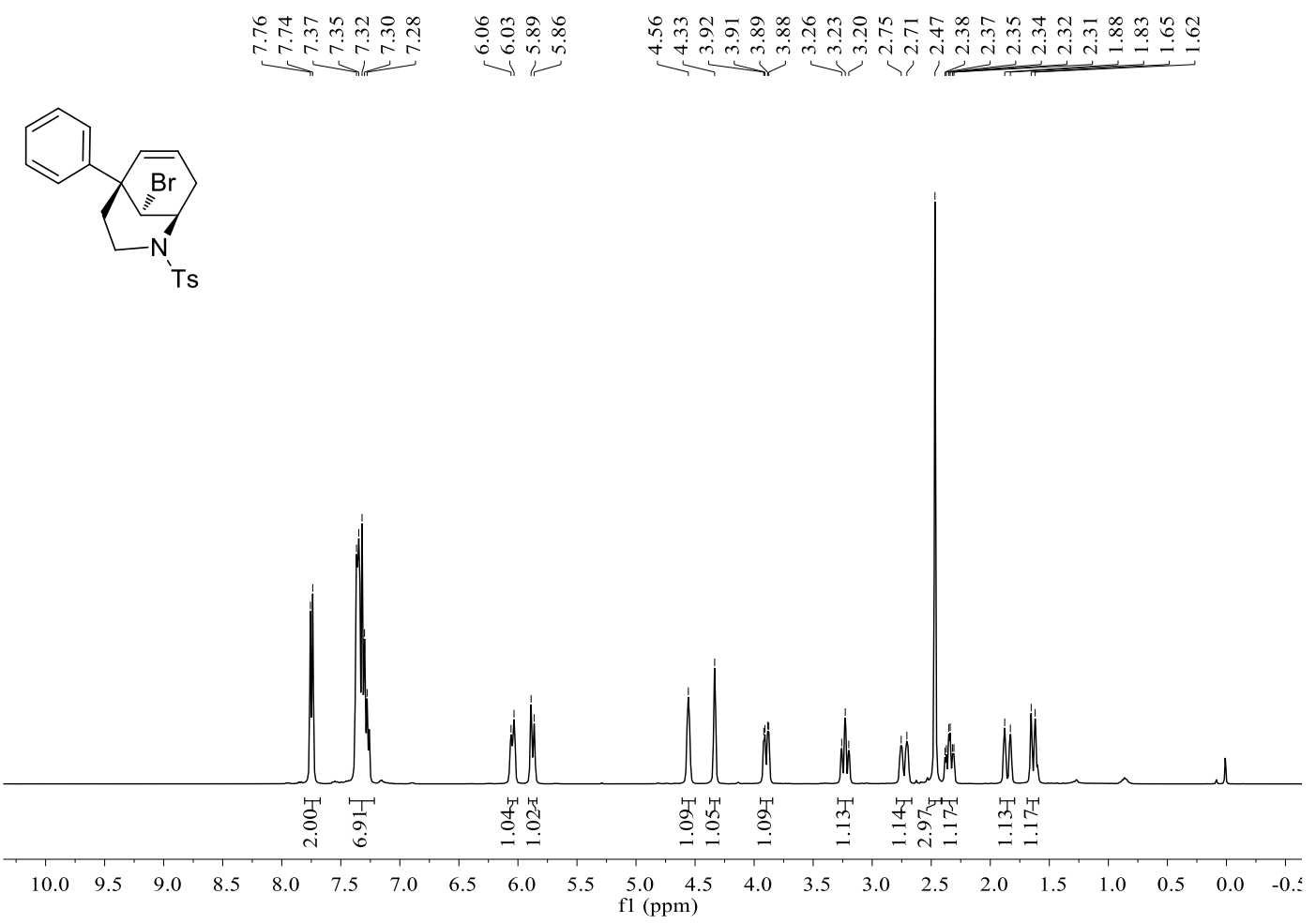

${ }^{13} \mathrm{C}$ NMR of 2a' (100 $\left.\mathrm{MHz}, \mathrm{CDCl}_{3}\right)$

प̛

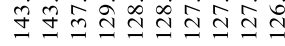

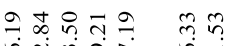

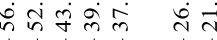
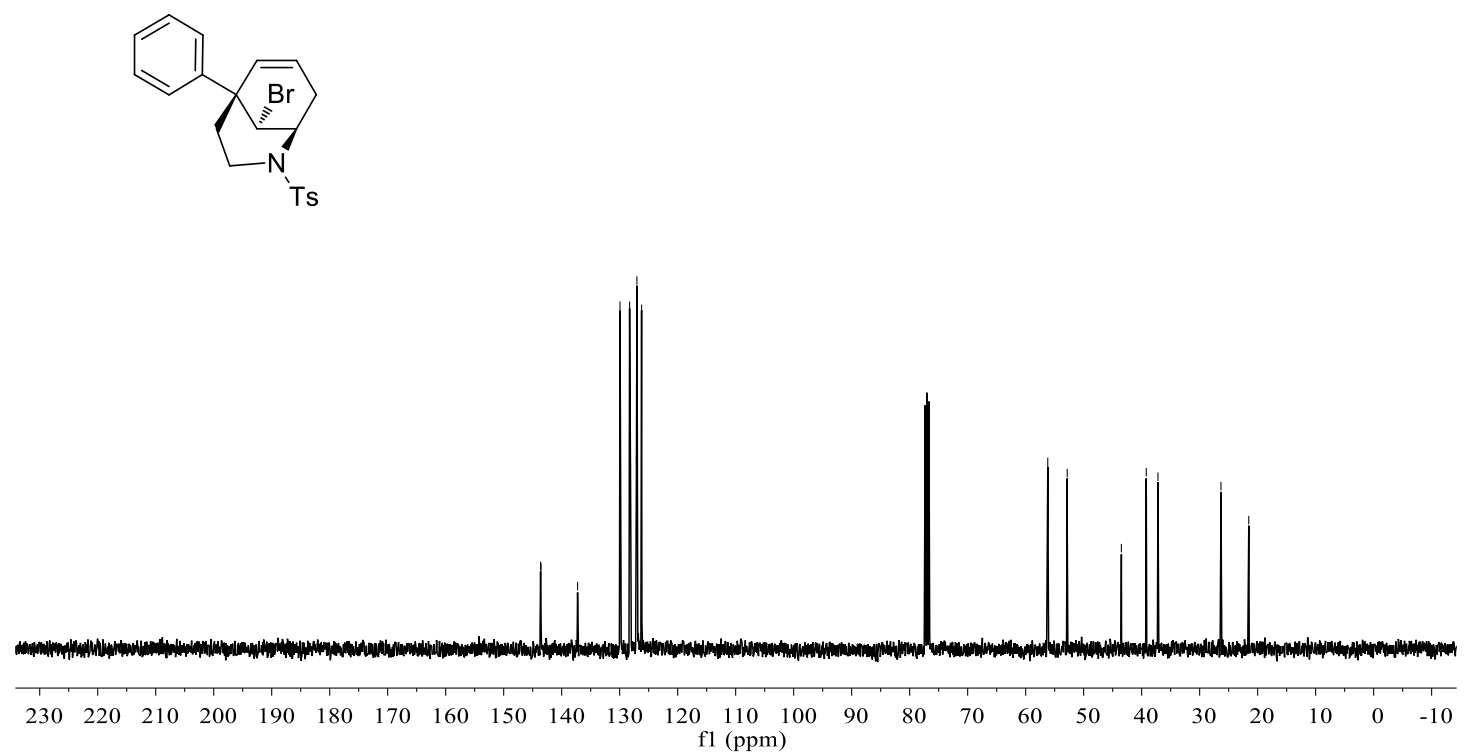


\section{${ }^{1} \mathrm{H}$ NMR of $2 \mathrm{~b}\left(400 \mathrm{MHz}, \mathrm{CDCl}_{3}\right)$}

$\underbrace{\infty}$

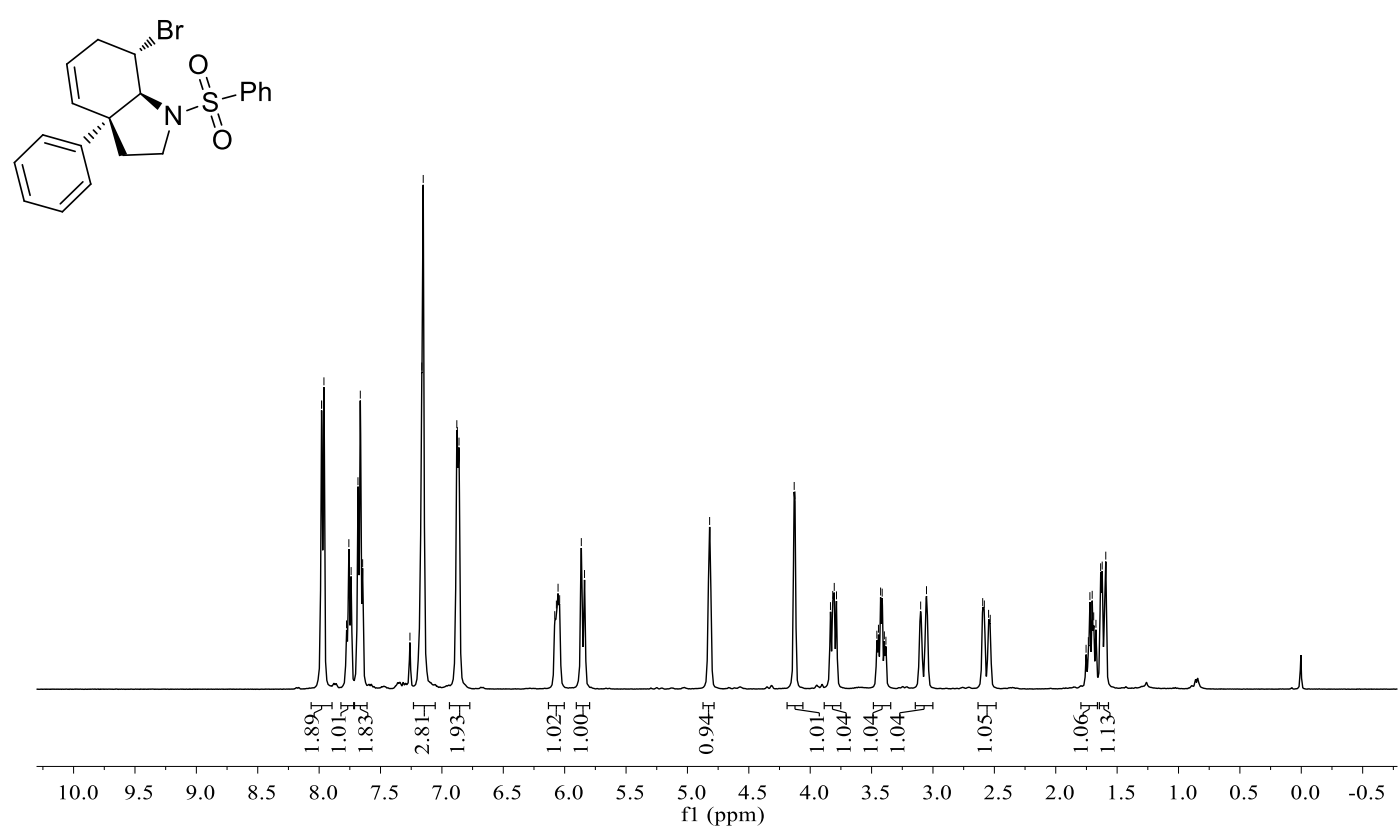

${ }^{13} \mathrm{C}$ NMR of $2 \mathrm{~b}\left(100 \mathrm{MHz}, \mathrm{CDCl}_{3}\right)$

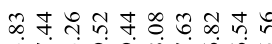

$\underline{\underline{2}}$

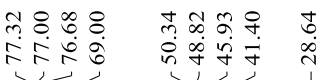<smiles>O=S(=O)(c1ccccc1)N1CCC2(c3ccccc3)C=CCC(Br)C12</smiles>

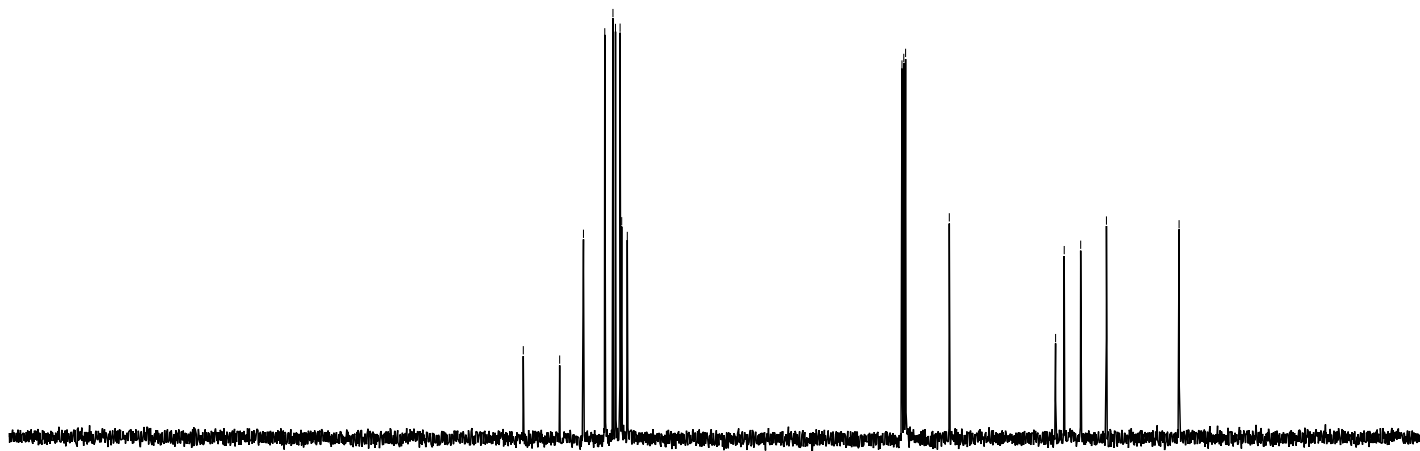

$\begin{array}{llllllllllllllllllllllllllllllll}230 & 220 & 210 & 200 & 190 & 180 & 170 & 160 & 150 & 140 & 130 & 120 & 110 & 100 & 90 & 80 & 70 & 60 & 50 & 40 & 30 & 20 & 10 & 0 & -10\end{array}$ 


\section{${ }^{1} \mathrm{H}$ NMR of $2 \mathrm{c}\left(400 \mathrm{MHz}, \mathrm{CDCl}_{3}\right)$}

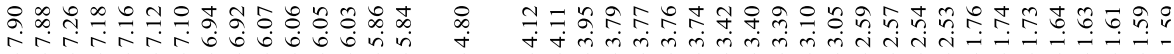<smiles>COc1ccc(S(=O)(=O)N2CCC3(c4ccccc4)C=CCC(Br)C23)cc1</smiles>

${ }^{13} \mathrm{C}$ NMR of $2 \mathrm{c}\left(100 \mathrm{MHz}, \mathrm{CDCl}_{3}\right)$

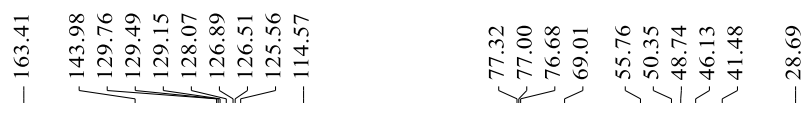<smiles>COc1ccc(S(=O)(=O)N2CCC3(c4ccccc4)C=CCC(Br)C23)cc1</smiles>

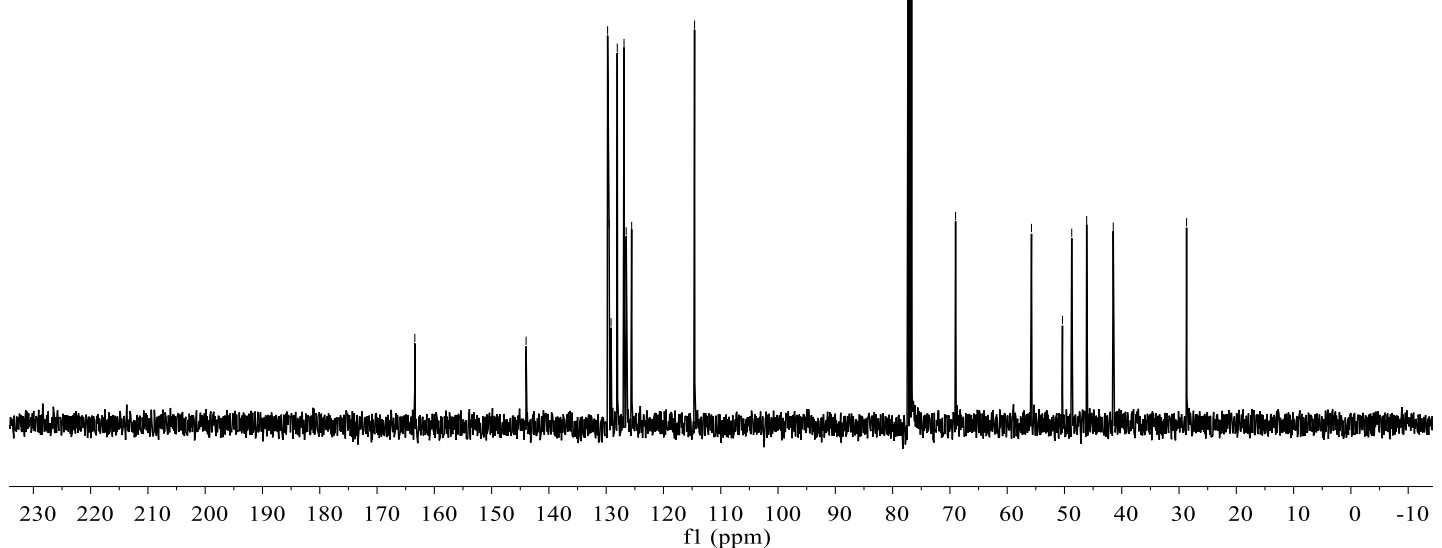


${ }^{1} \mathrm{H}$ NMR of $2 \mathrm{~d}\left(400 \mathrm{MHz}, \mathrm{CDCl}_{3}\right)$

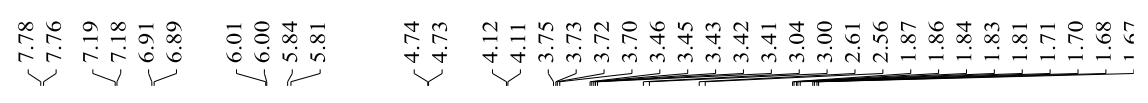

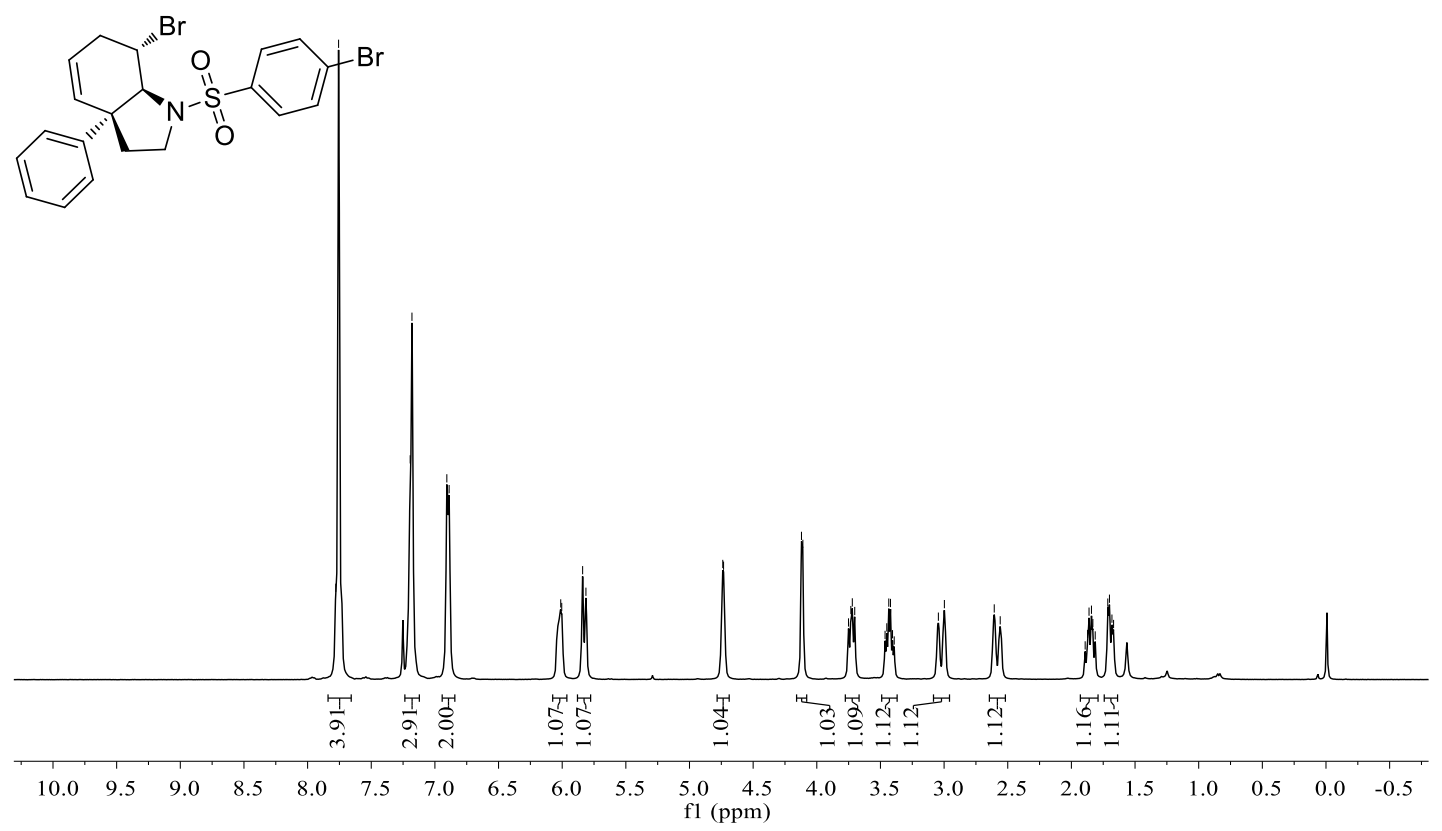

${ }^{13} \mathrm{C}$ NMR of $2 \mathrm{~d}\left(100 \mathrm{MHz}, \mathrm{CDCl}_{3}\right)$

के में 웅 की

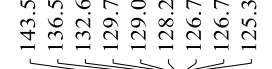

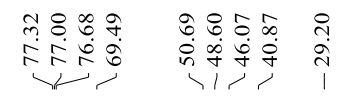<smiles>O=S(=O)(c1ccc(Br)cc1)N1CCC2(c3ccccc3)C=CC[C@@H](Br)C12</smiles>

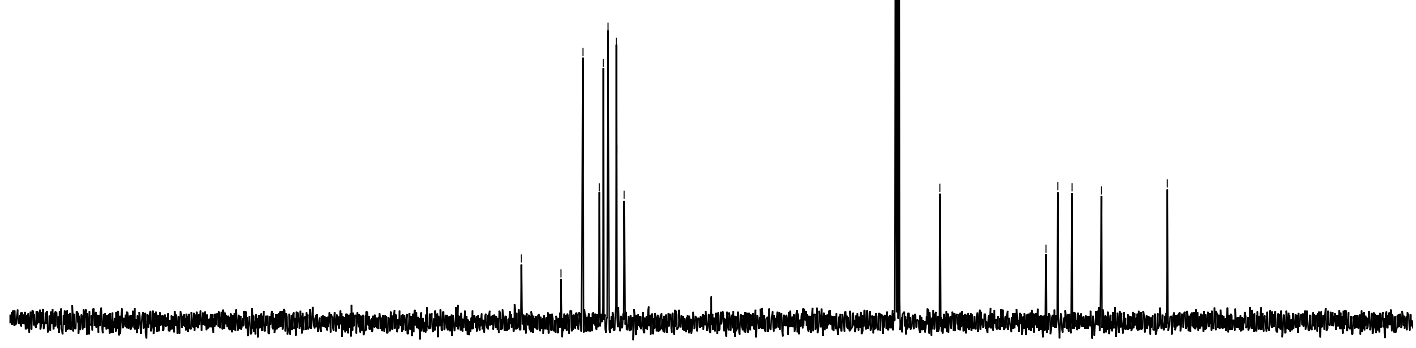

$\begin{array}{rlllllllllllllllllllllllllllll}230 & 220 & 210 & 200 & 190 & 180 & 170 & 160 & 150 & 140 & 130 & 120 & 110 & 100 & 90 & 80 & 70 & 60 & 50 & 40 & 30 & 20 & 10 & 0 & -10\end{array}$ 
${ }^{1} \mathrm{H}$ NMR of $2 \mathrm{e}\left(400 \mathrm{MHz}, \mathrm{CDCl}_{3}\right)$

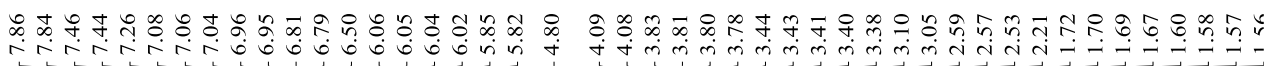<smiles>Cc1cccc(C23C=CCC(Br)C2N([135I])CC3)c1</smiles>

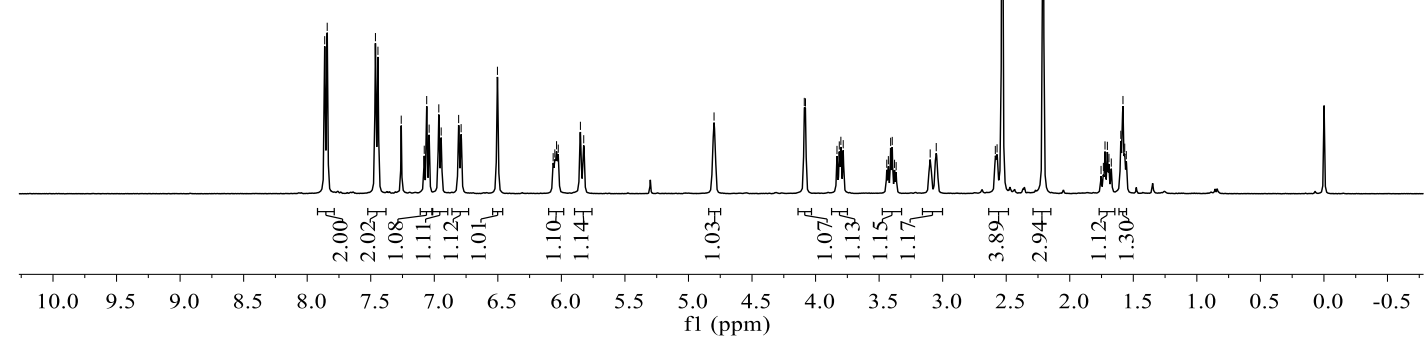

${ }^{13} \mathrm{C}$ NMR of $2 \mathrm{e}\left(100 \mathrm{MHz}, \mathrm{CDCl}_{3}\right)$

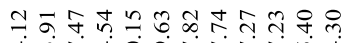

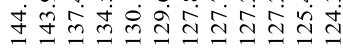

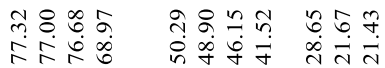<smiles>Cc1cccc(C23C=CCC(Br)C2N([135I])CC3)c1</smiles>

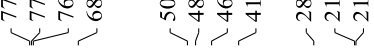

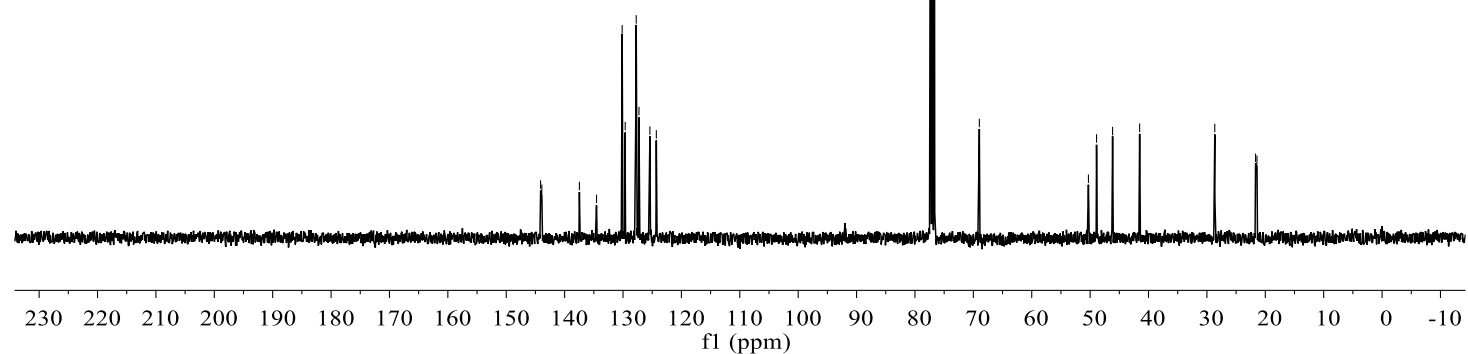


${ }^{1} \mathrm{H}$ NMR of $2 \mathrm{f}\left(400 \mathrm{MHz}, \mathrm{CDCl}_{3}\right)$

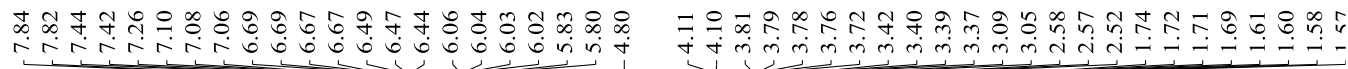<smiles>COc1cccc(C23C=CCC(Br)C2N([13I])CC3)c1</smiles>

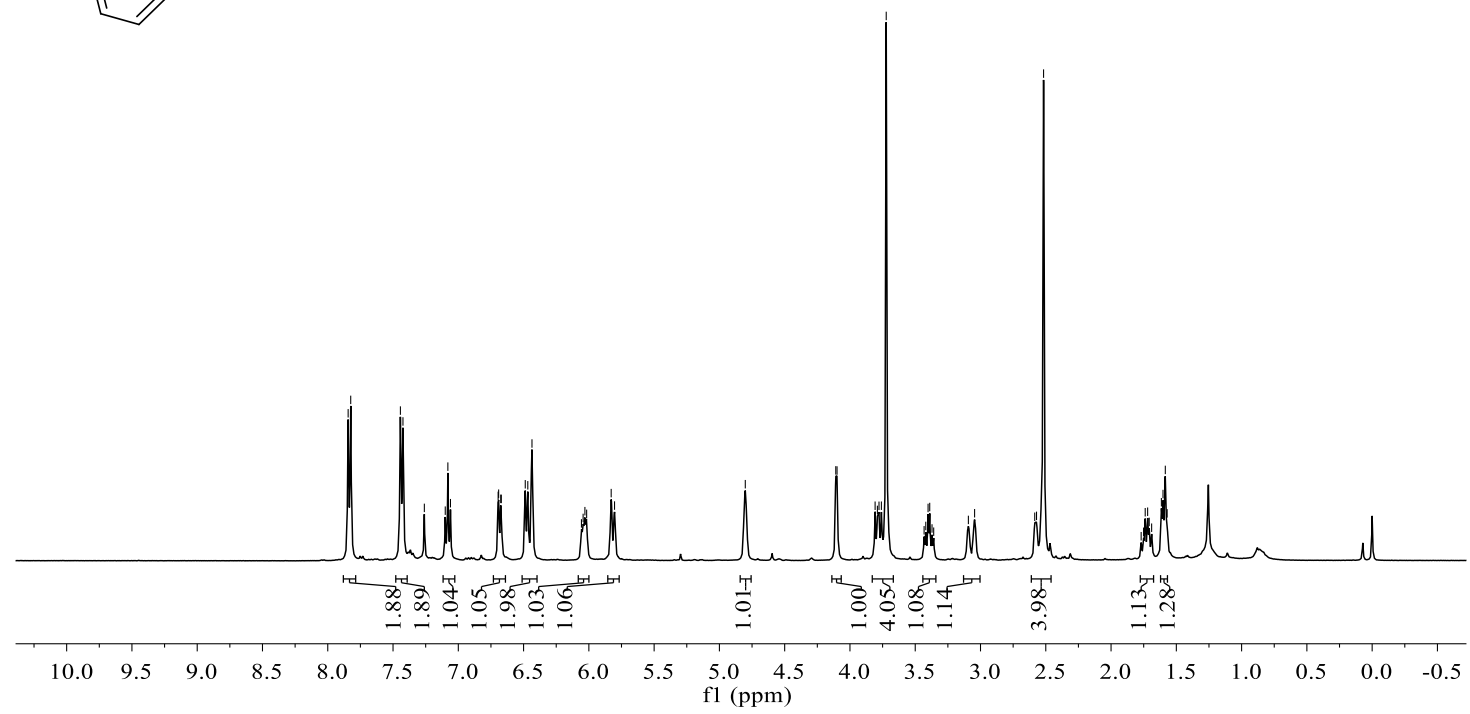

${ }^{13} \mathrm{C}$ NMR of $2 \mathrm{f}\left(100 \mathrm{MHz}, \mathrm{CDCl}_{3}\right)$

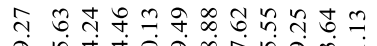

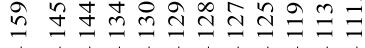

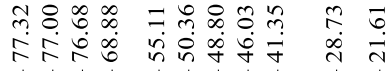<smiles>COc1cccc([C@@]2(C)CCN([As])[C@@H]2C)c1</smiles>

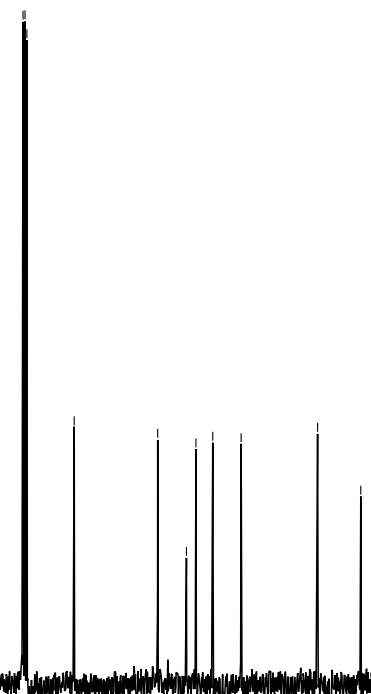

$\begin{array}{llllllllllllllllllllllllll}230 & 220 & 210 & 200 & 190 & 180 & 170 & 160 & 150 & 140 & 130 & 120 & 110 & 100 & 90 & 80 & 70 & 60 & 50 & 40 & 30 & 20 & 10 & 0 & -10\end{array}$ 
${ }^{1} \mathrm{H}$ NMR of $2 \mathrm{~g}\left(400 \mathrm{MHz}, \mathrm{CDCl}_{3}\right)$

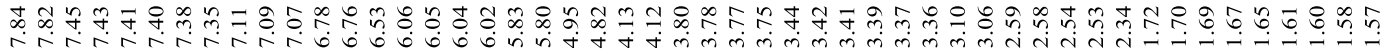<smiles>CN1CCC(c2ccccc2)(c2cccc(OC(C)(F)F)c2)CC1Br</smiles>

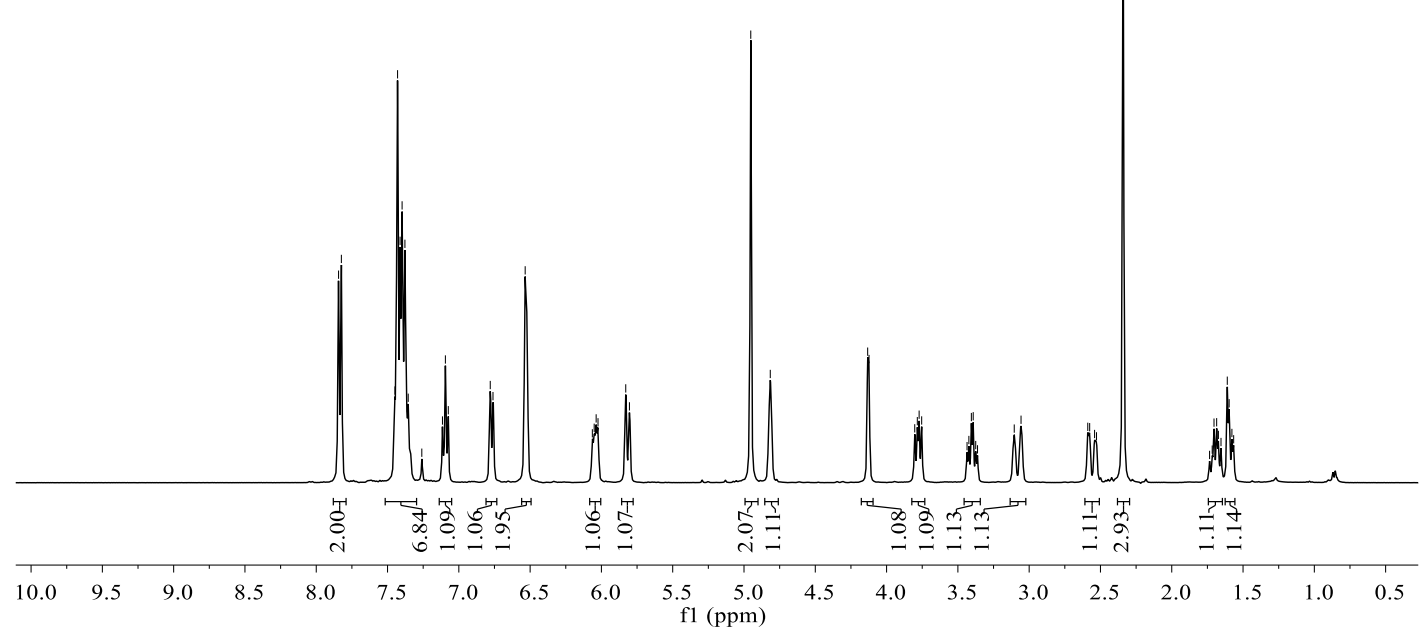

${ }^{13} \mathrm{C}$ NMR of $2 \mathrm{~g}\left(100 \mathrm{MHz}, \mathrm{CDCl}_{3}\right)$

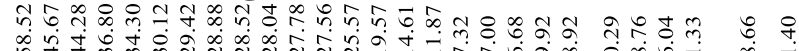

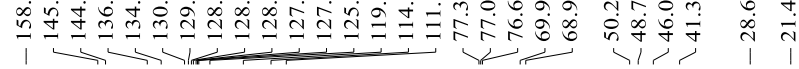

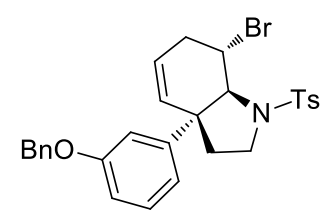

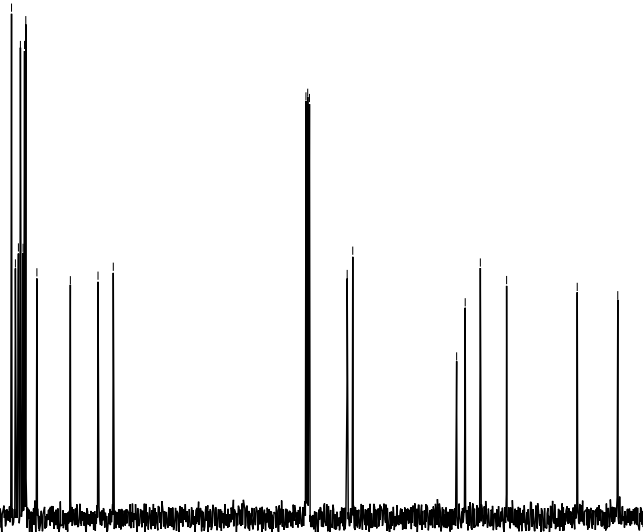

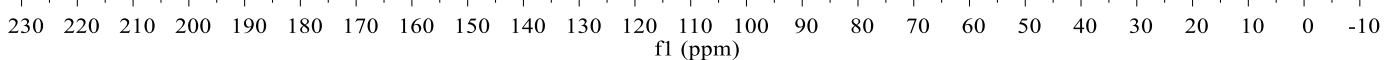


${ }^{1} \mathrm{H}$ NMR of $2 \mathrm{~h}\left(400 \mathrm{MHz}, \mathrm{CDCl}_{3}\right)$

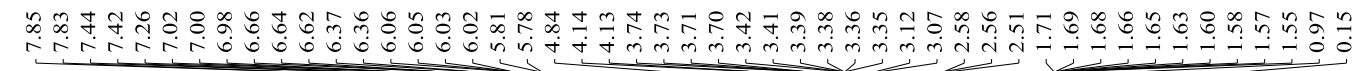<smiles>CCCCOc1cccc([C@]2(CC)CC=CC(Br)C2[NH3+])c1</smiles>

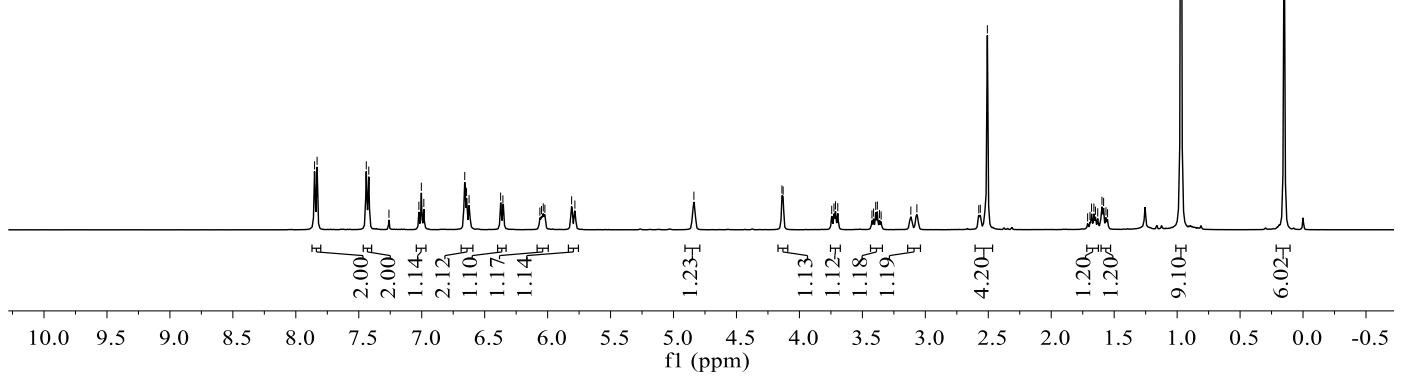

${ }^{13} \mathrm{C}$ NMR of $2 \mathrm{~h}\left(100 \mathrm{MHz}, \mathrm{CDCl}_{3}\right)$

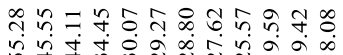

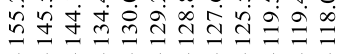

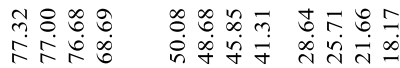

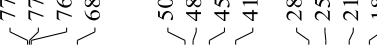

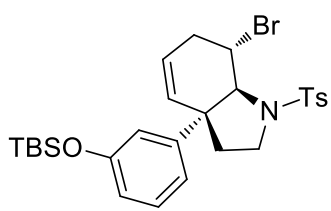

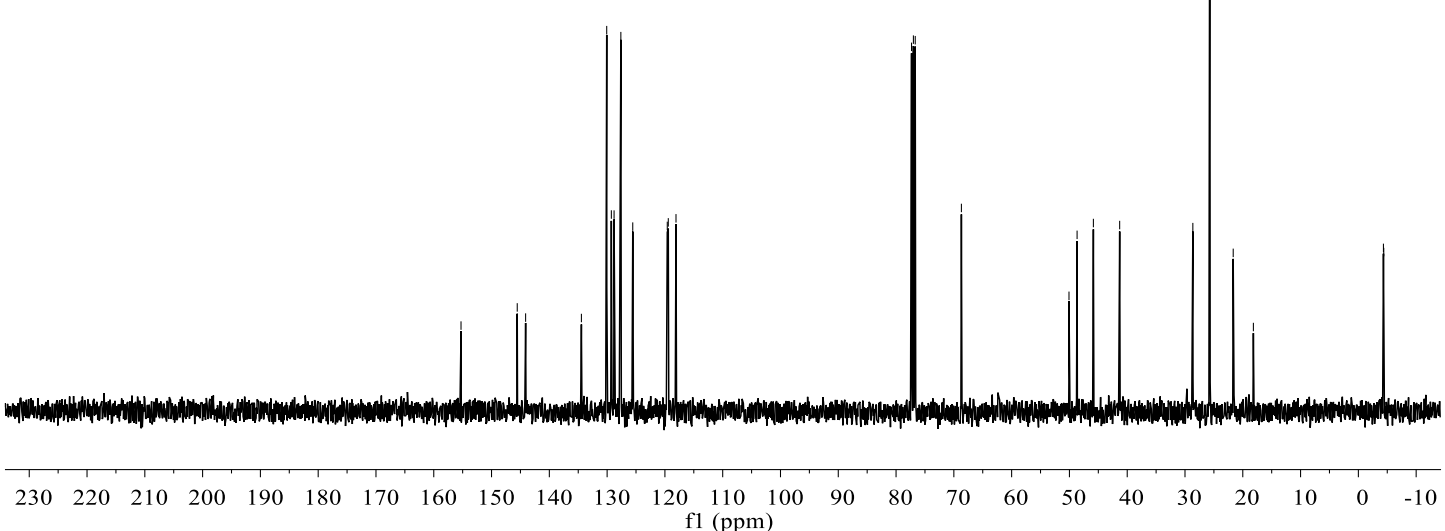


${ }^{1} \mathrm{H}$ NMR of $2 \mathrm{i}\left(400 \mathrm{MHz}, \mathrm{CDCl}_{3}\right)$

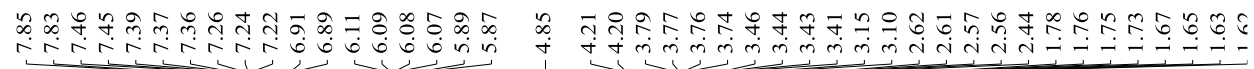

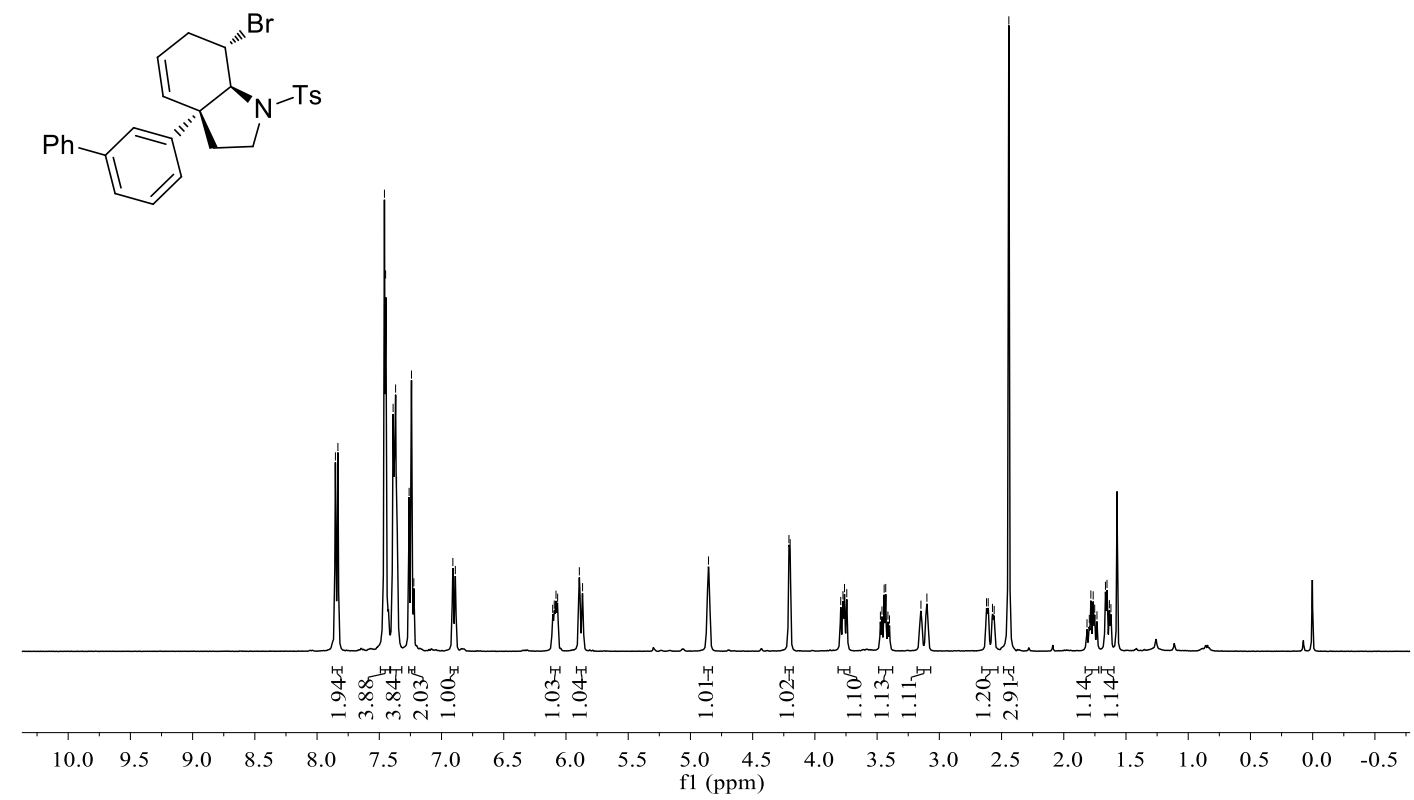

${ }^{13} \mathrm{C}$ NMR of $2 \mathrm{i}\left(100 \mathrm{MHz}, \mathrm{CDCl}_{3}\right)$

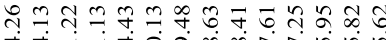

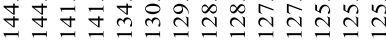

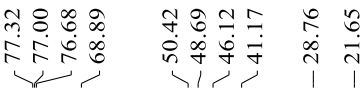

(Br

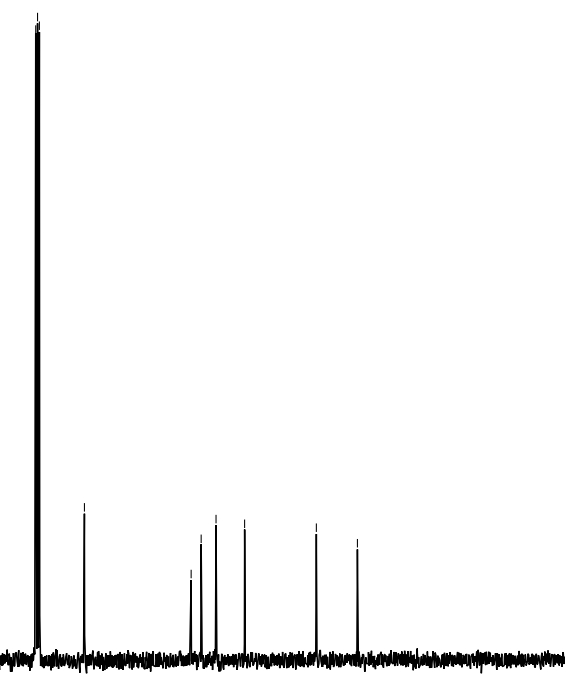

$\begin{array}{lllllllllllllllllllllllll}230 & 220 & 210 & 200 & 190 & 180 & 170 & 160 & 150 & 140 & 130 & 120 & 110 & 100 & 90 & 80 & 70 & 60 & 50 & 40 & 30 & 20 & 10 & 0 & -10\end{array}$ 
${ }^{1} \mathrm{H}$ NMR of $2 \mathrm{j}\left(400 \mathrm{MHz}, \mathrm{CDCl}_{3}\right)$

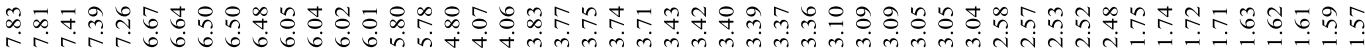<smiles>COc1ccc([C@]23C=CCC(Br)C2N([13F])CC3)cc1OC</smiles>

${ }^{13} \mathrm{C}$ NMR of $2 \mathrm{j}\left(100 \mathrm{MHz}, \mathrm{CDCl}_{3}\right)$

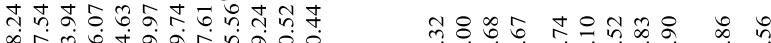

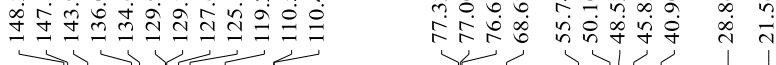<smiles></smiles>

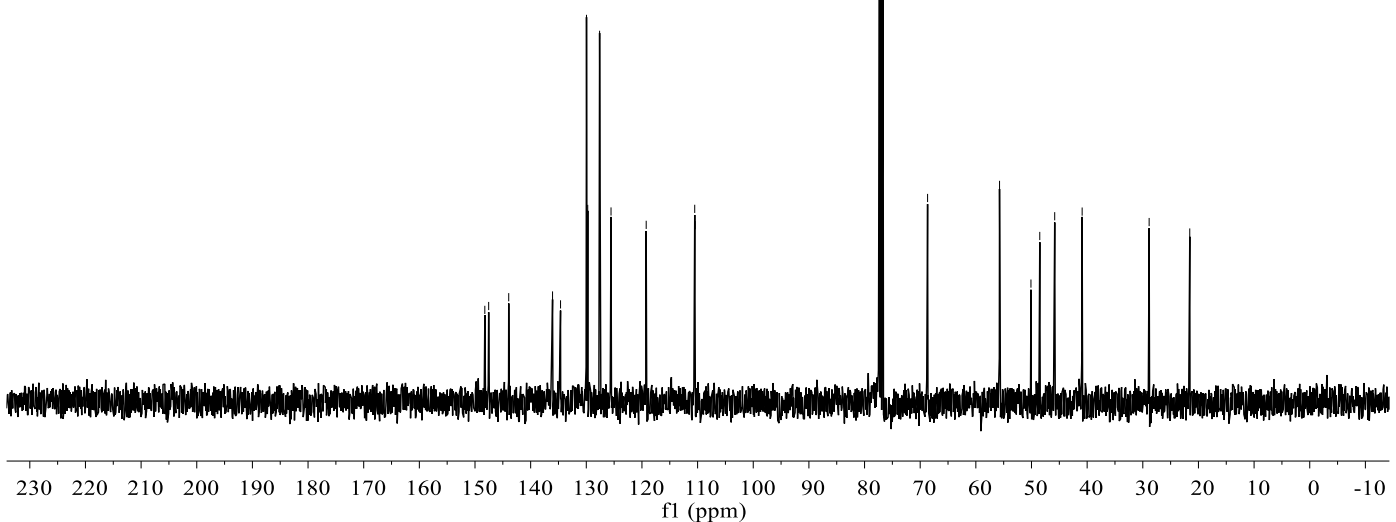


${ }^{1} \mathrm{H}$ NMR of 2k (400 MHz, $\left.\mathrm{CDCl}_{3}\right)$

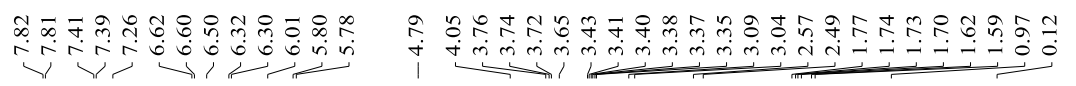

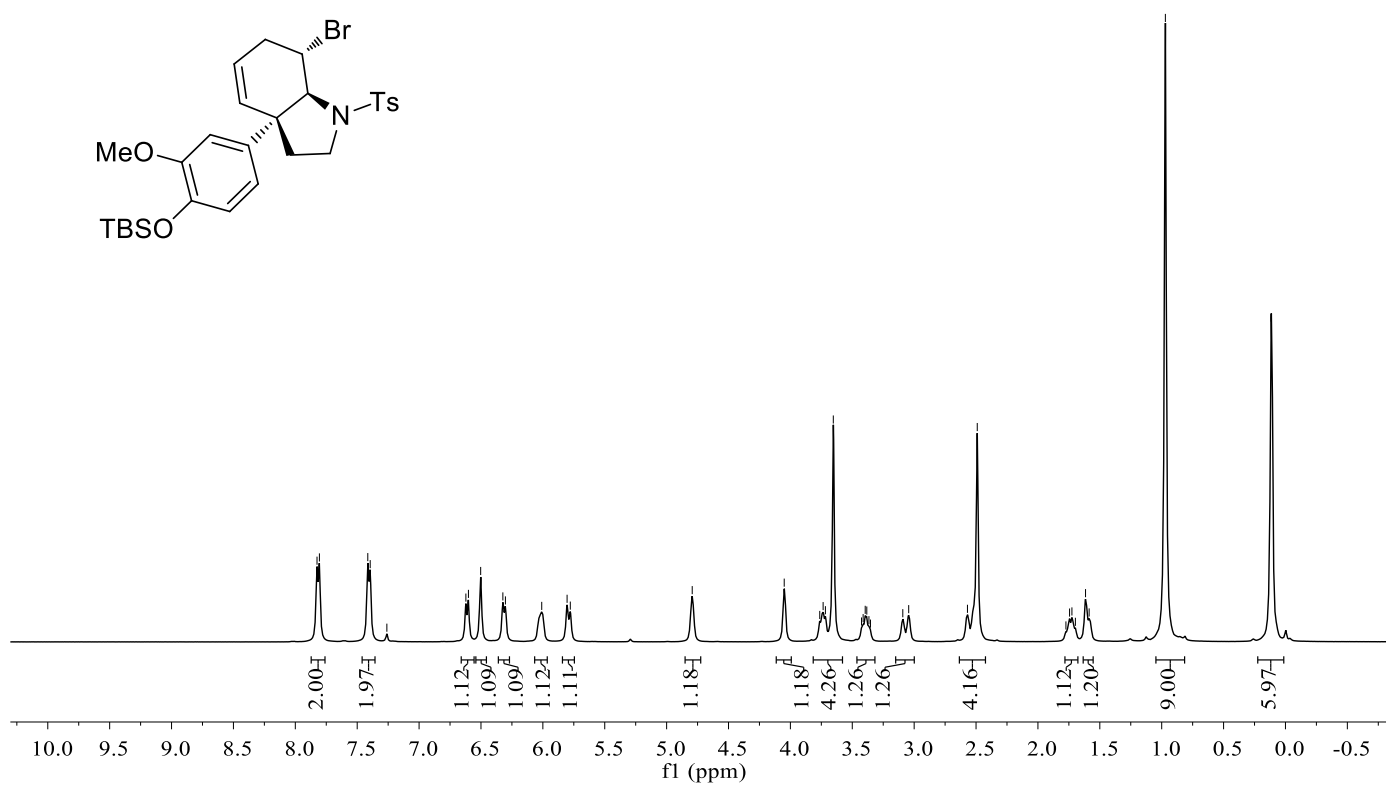

${ }^{13} \mathrm{C}$ NMR of $2 \mathrm{k}\left(100 \mathrm{MHz}, \mathrm{CDCl}_{3}\right)$

خิ

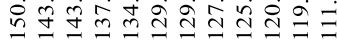

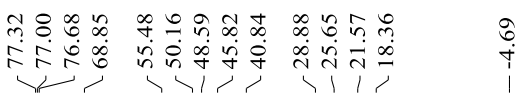<smiles>CCCOc1ccc(C23C=CCC(Br)C2N([As])CC3)cc1OC</smiles>

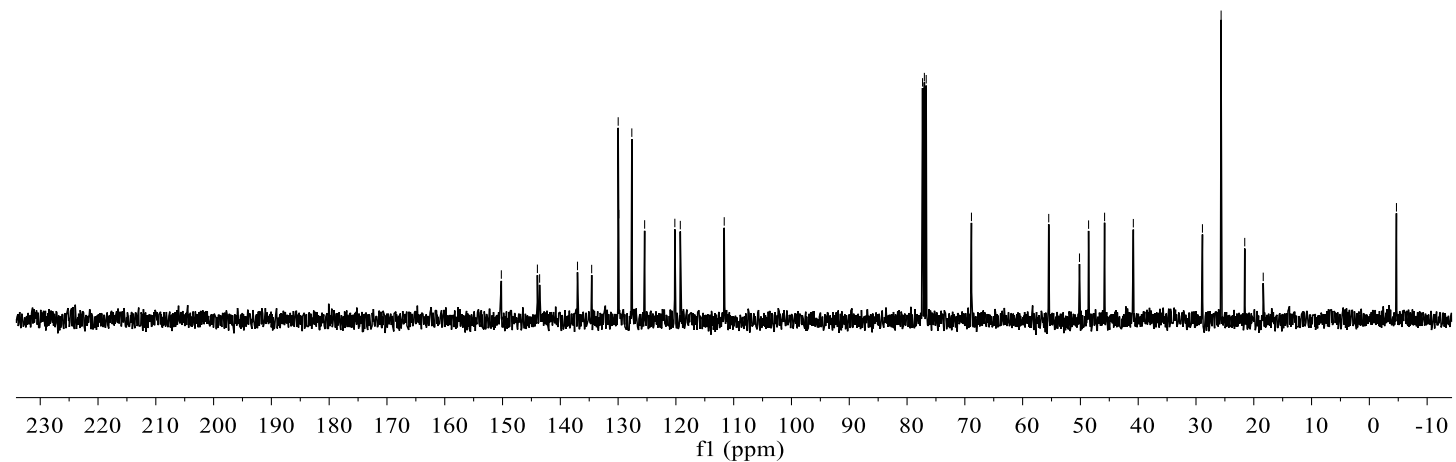


${ }^{1} \mathrm{H}$ NMR of $2 \mathrm{l}\left(400 \mathrm{MHz}, \mathrm{CDCl}_{3}\right)$

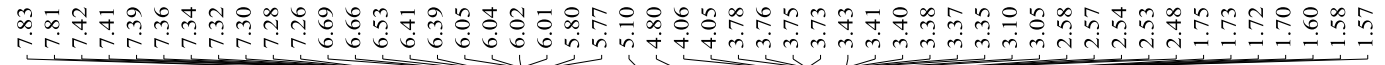

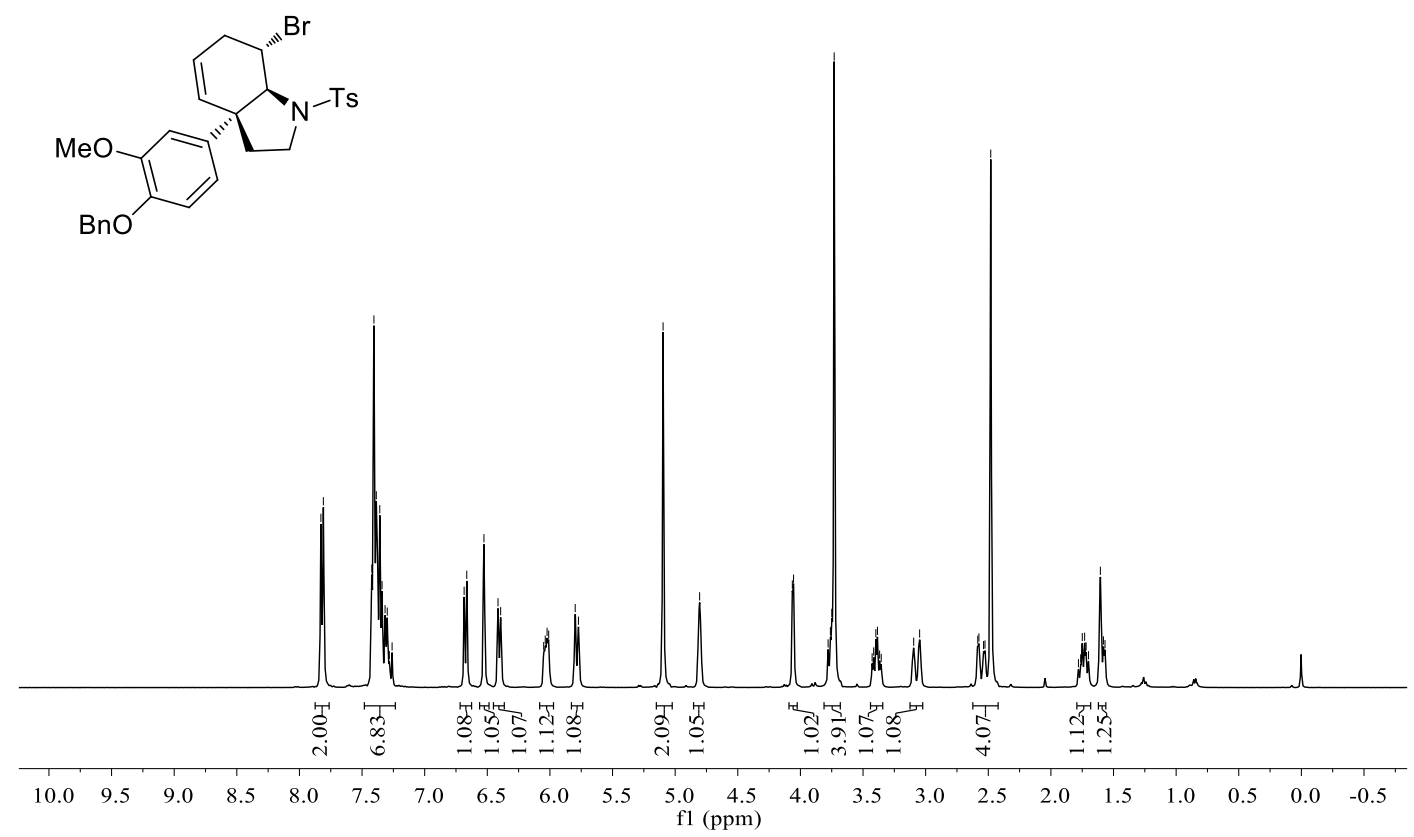

${ }^{13} \mathrm{C}$ NMR of $2 \mathrm{l}\left(100 \mathrm{MHz}, \mathrm{CDCl}_{3}\right)$

б.

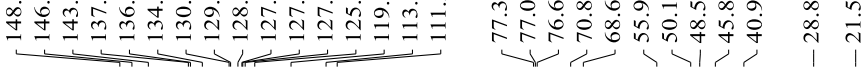
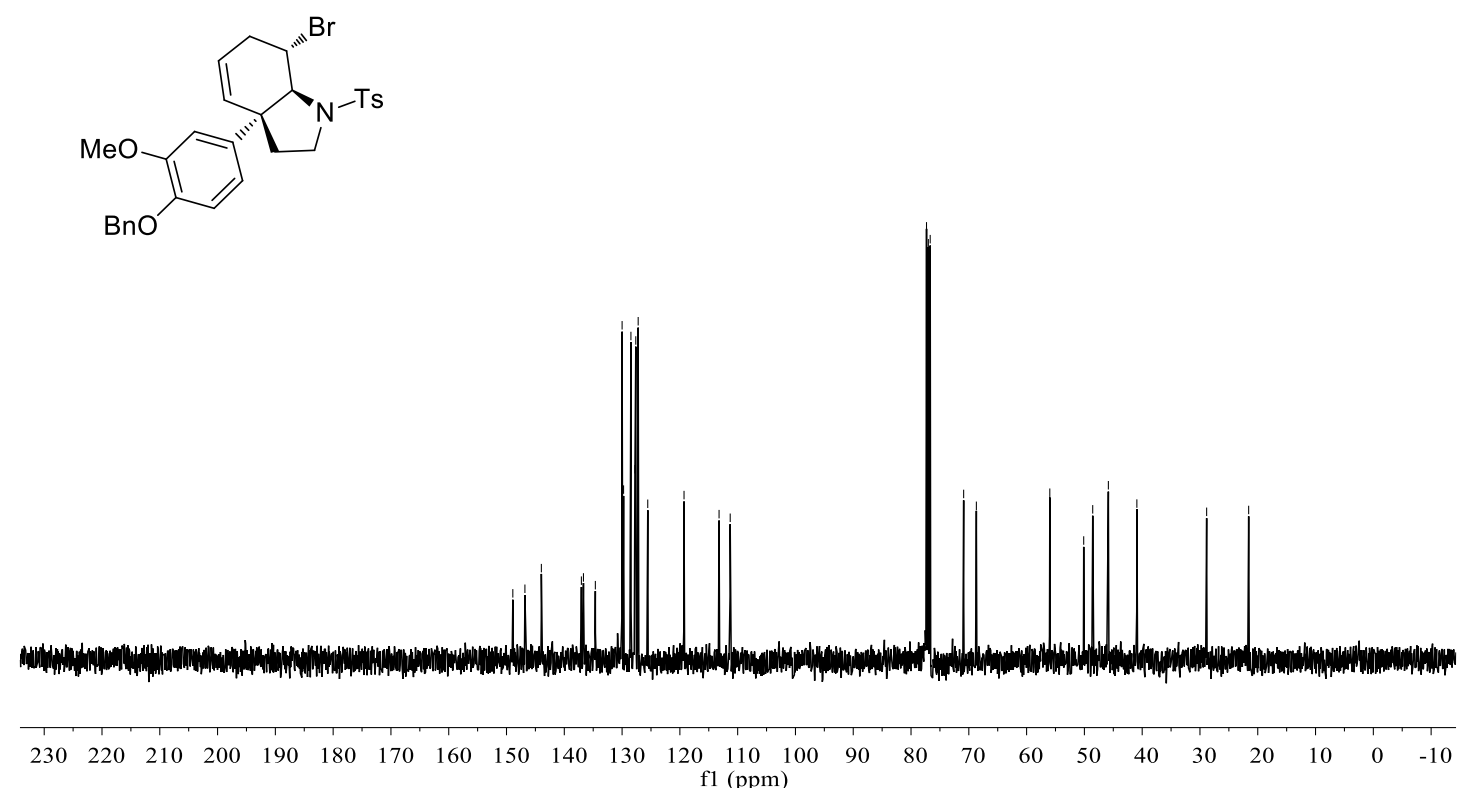
${ }^{1} \mathrm{H}$ NMR of $2 \mathrm{~m}\left(400 \mathrm{MHz}, \mathrm{CDCl}_{3}\right)$

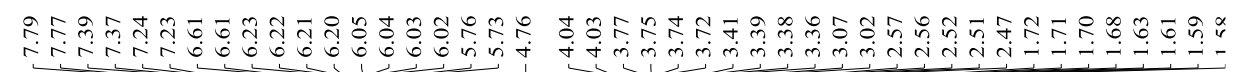<smiles>COc1cc(C23C=CCC(Br)C2N([135I])CC3)ccc1Br</smiles>

\section{${ }^{13} \mathrm{C}$ NMR of $2 \mathrm{~m}\left(100 \mathrm{MHz}, \mathrm{CDCl}_{3}\right)$}

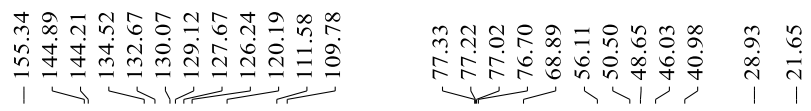<smiles>COc1ccc(C23C=CCC(Br)C2N([135I])CC3)cc1Br</smiles>

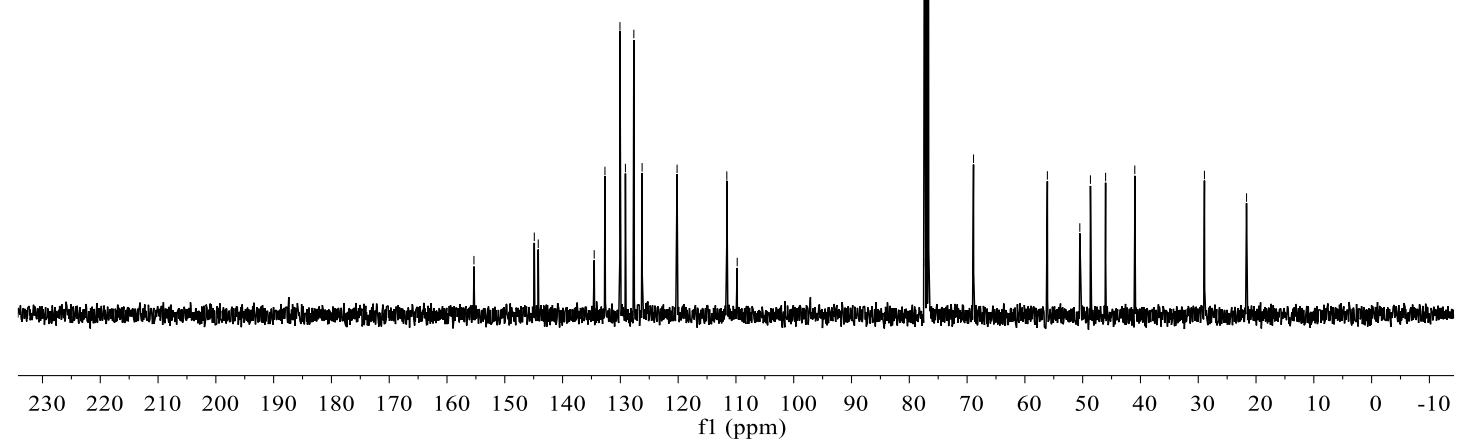


${ }^{1} \mathrm{H}$ NMR of 2n (400 MHz, $\left.\mathrm{CDCl}_{3}\right)$

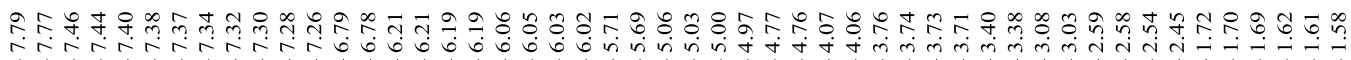<smiles>[NH3+]N1CC[C@]2(c3ccc(Br)c(OCBr)c3)C=CCC(Br)C12</smiles>

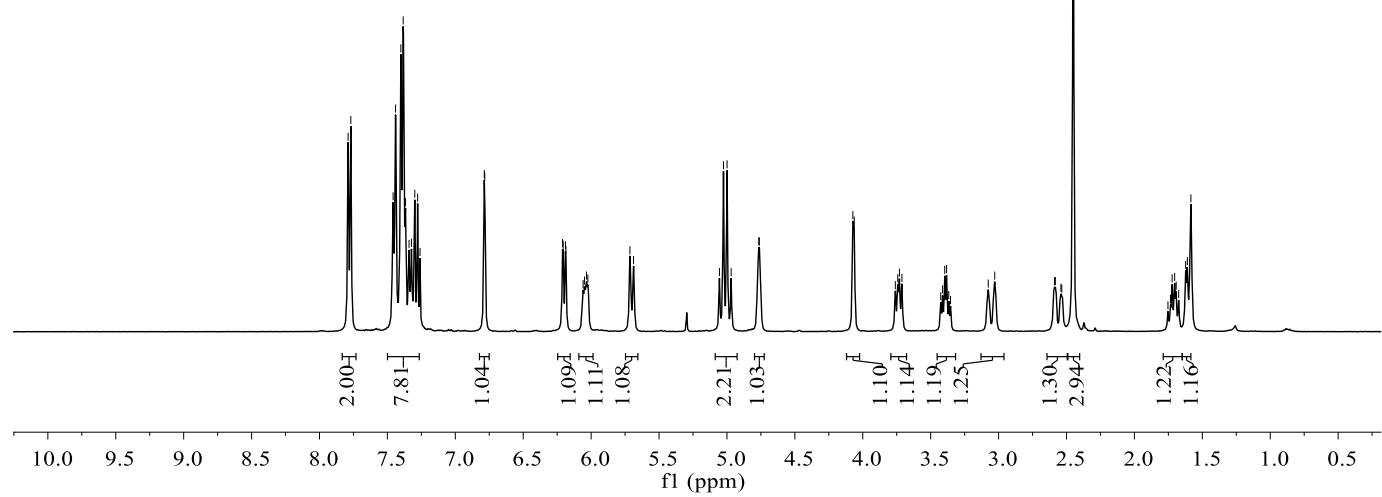

${ }^{13} \mathrm{C}$ NMR of $2 \mathrm{n}\left(100 \mathrm{MHz}, \mathrm{CDCl}_{3}\right)$

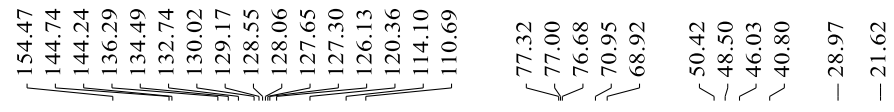<smiles>[B]Oc1cc([C@]23C=CCC(Br)C2N([NH3+])CC3)ccc1Br</smiles>

$\begin{array}{lllllllllllllllllllllllll}230 & 220 & 210 & 200 & 190 & 180 & 170 & 160 & 150 & 140 & 130 & 120 & 110 & 100 & 90 & 80 & 70 & 60 & 50 & 40 & 30 & 20 & 10 & 0 & -10\end{array}$ 
${ }^{1} \mathrm{H}$ NMR of $20\left(400 \mathrm{MHz}, \mathrm{CDCl}_{3}\right)$

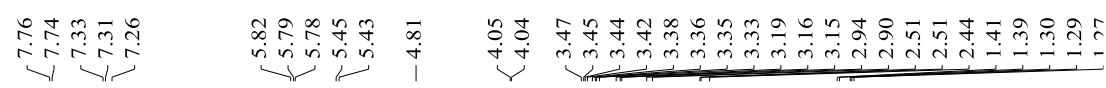<smiles>COC[C@]12C=CCC(Br)C1N([135I])CC2</smiles>

${ }^{13} \mathrm{C}$ NMR of $20\left(100 \mathrm{MHz}, \mathrm{CDCl}_{3}\right)$

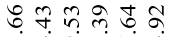

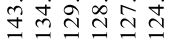

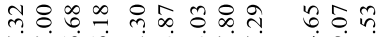

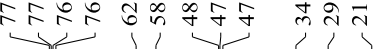<smiles>COCC12C=CCC(Br)C1N([135I])CC2</smiles>

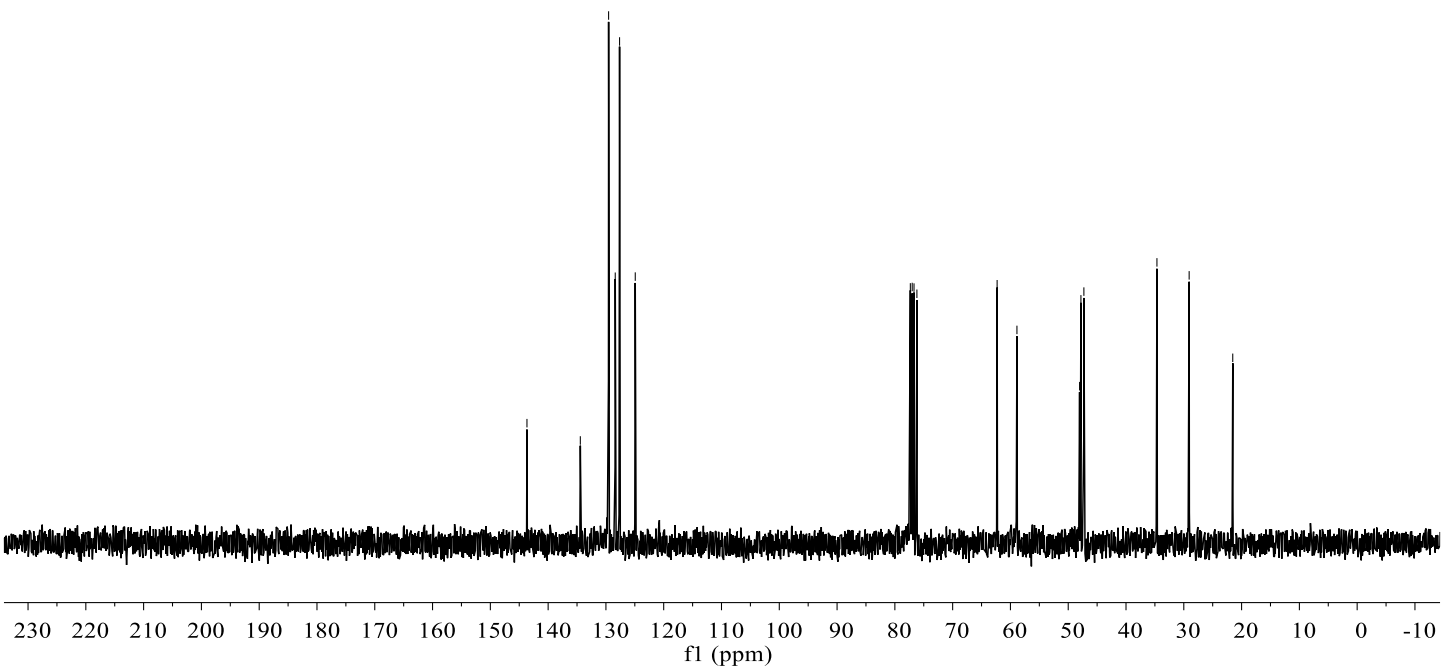


${ }^{1} \mathrm{H}$ NMR of 2p (400 MHz, $\left.\mathrm{CDCl}_{3}\right)$

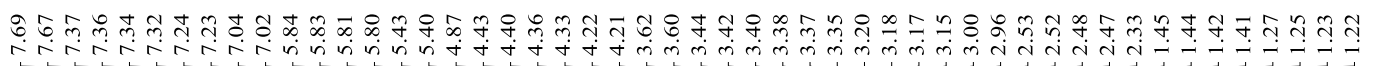

Br

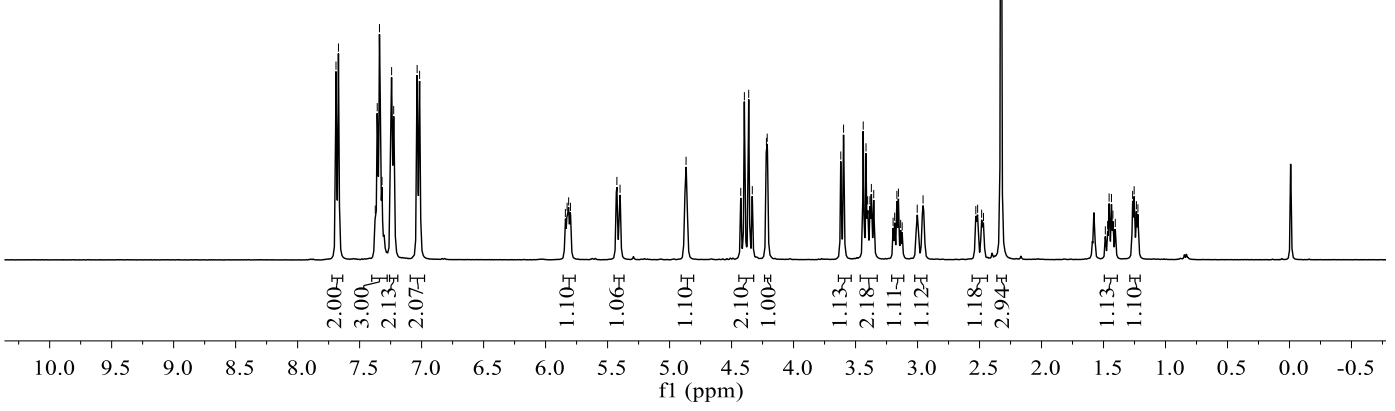

${ }^{13} \mathrm{C}$ NMR of $2 p\left(100 \mathrm{MHz}, \mathrm{CDCl}_{3}\right)$

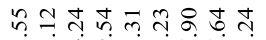

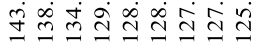

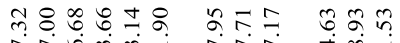

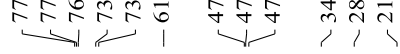

Bro-

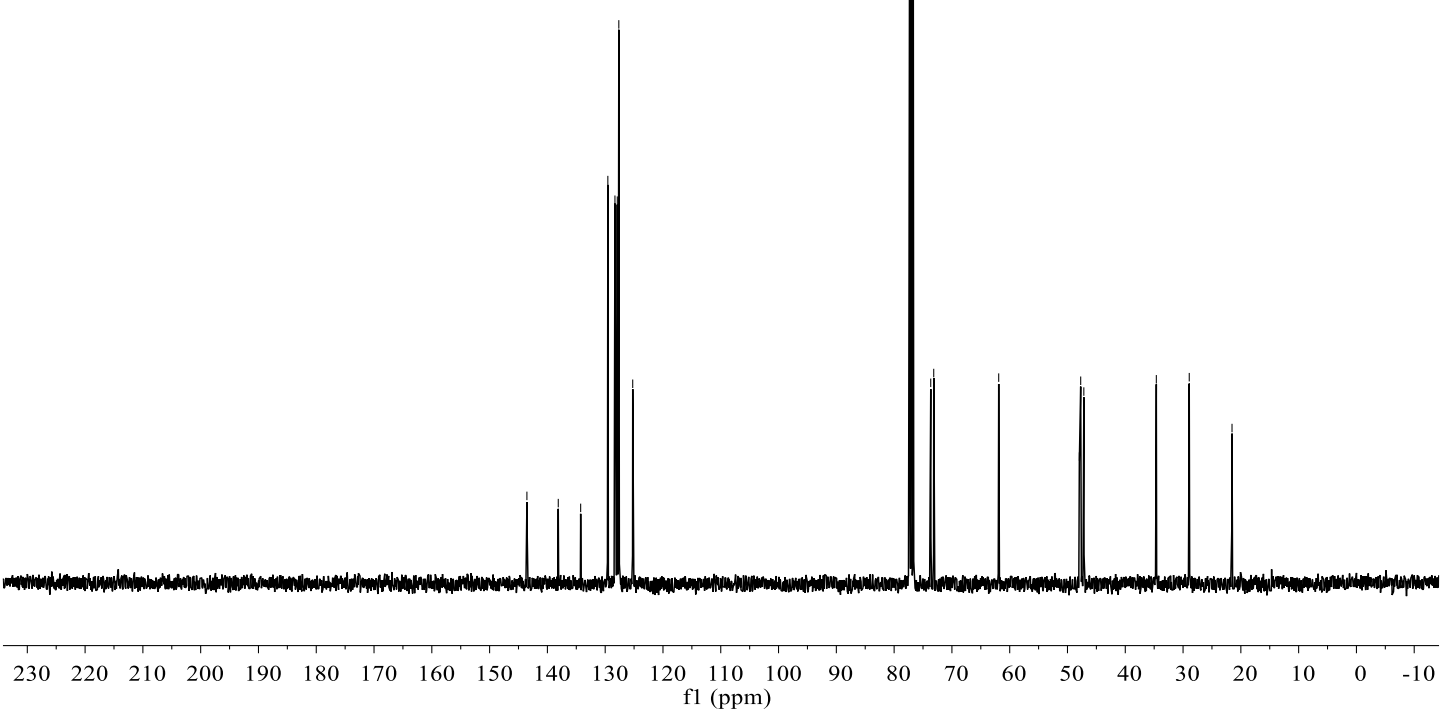


${ }^{1} \mathrm{H}$ NMR of $2 q\left(400 \mathrm{MHz}, \mathrm{CDCl}_{3}\right)$

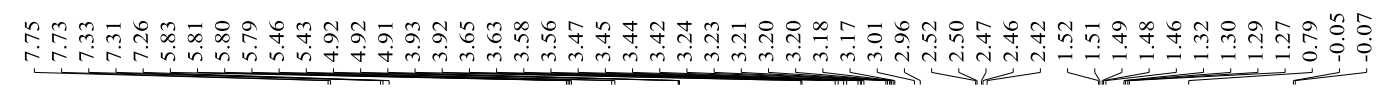<smiles>[Y5]N1CC[C@@H](COC(C)(C)C)C2C(Br)CC=C[C@@H]21</smiles>

${ }^{13} \mathrm{C}$ NMR of 2q (100 MHz, $\left.\mathrm{CDCl}_{3}\right)$

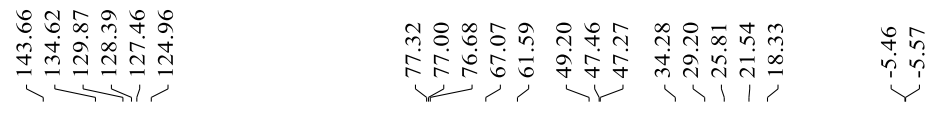

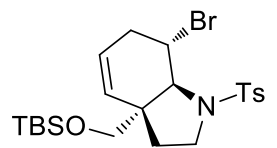

$\begin{array}{lllllllllllllllllllllllll}230 & 220 & 210 & 200 & 190 & 180 & 170 & 160 & 150 & 140 & 130 & \begin{array}{c}120 \\ \mathrm{f} 1(\mathrm{ppm})\end{array} & 100 & 90 & 80 & 70 & 60 & 50 & 40 & 30 & 20 & 10 & 0 & -10\end{array}$ 
${ }^{1} \mathrm{H}$ NMR of 2r (400 $\left.\mathrm{MHz}, \mathrm{CDCl}_{3}\right)$

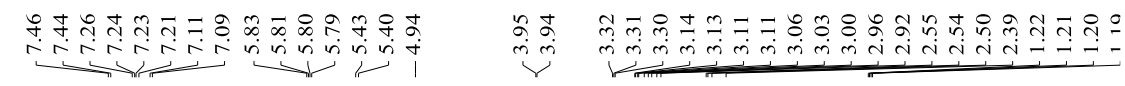
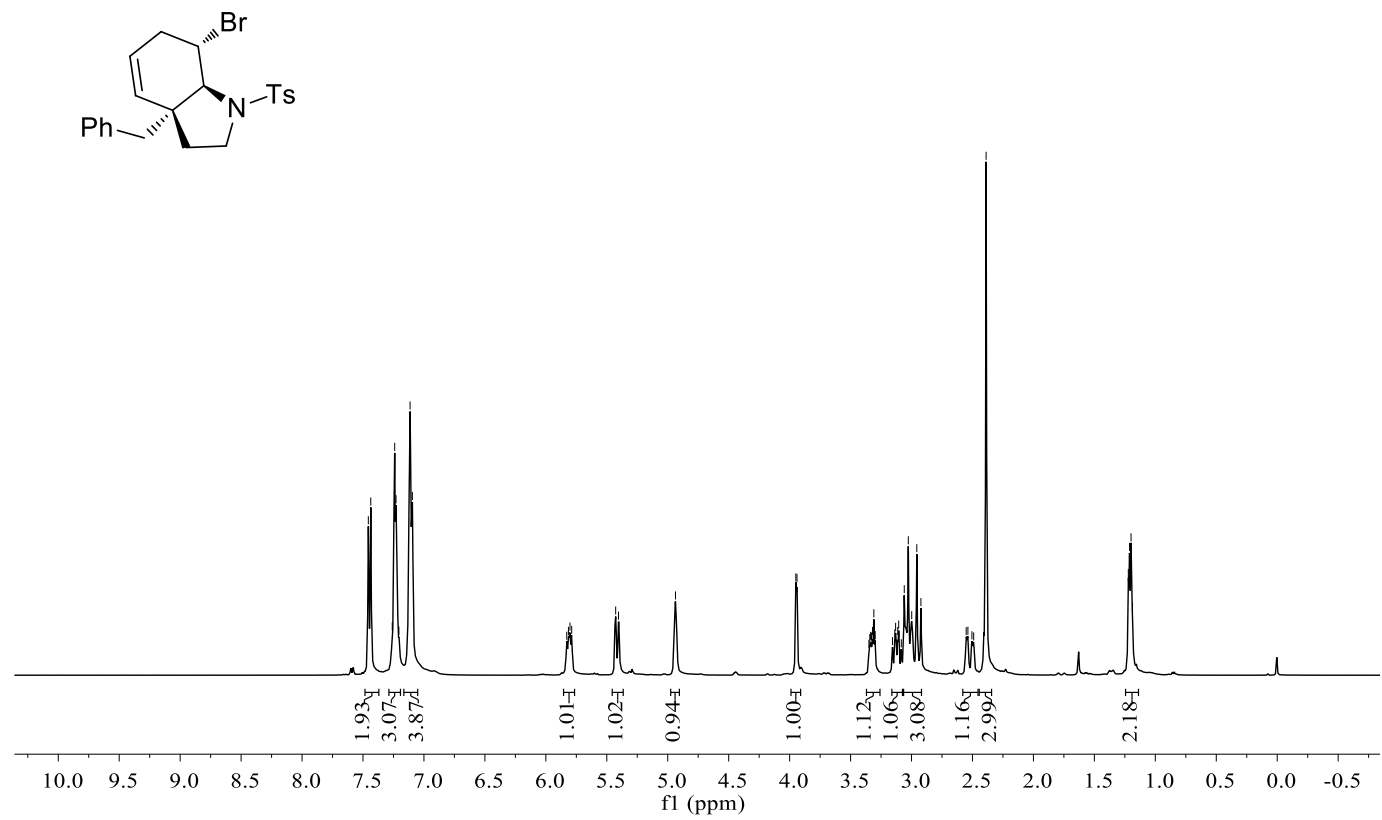

${ }^{13} \mathrm{C}$ NMR of 2 r (100 $\left.\mathrm{MHz}, \mathrm{CDCl}_{3}\right)$

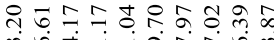

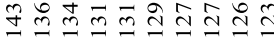

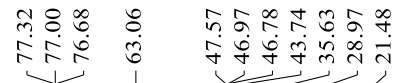

Pr

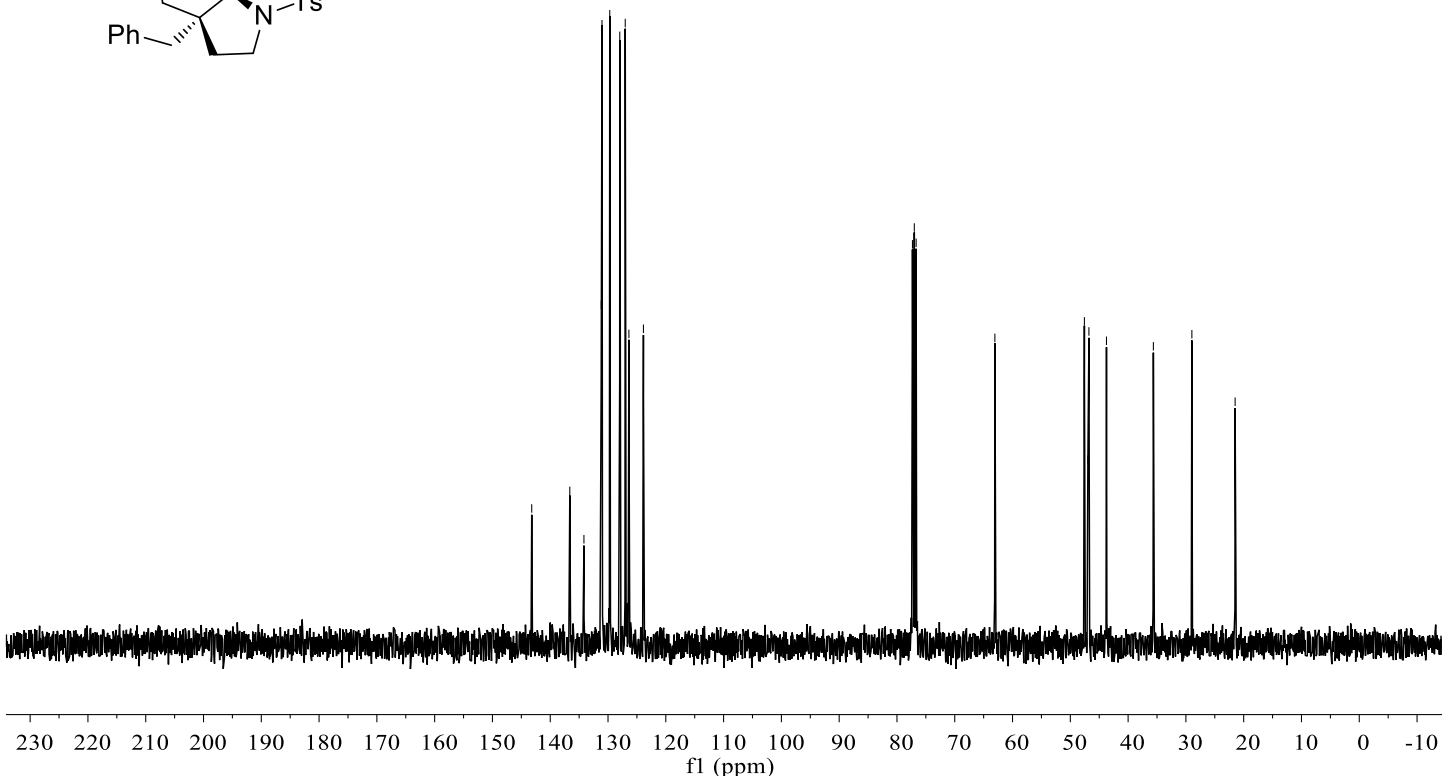


${ }^{1} \mathrm{H}$ NMR of 2s (400 MHz, $\left.\mathrm{CDCl}_{3}\right)$

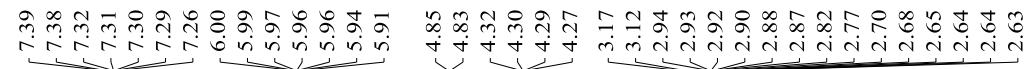

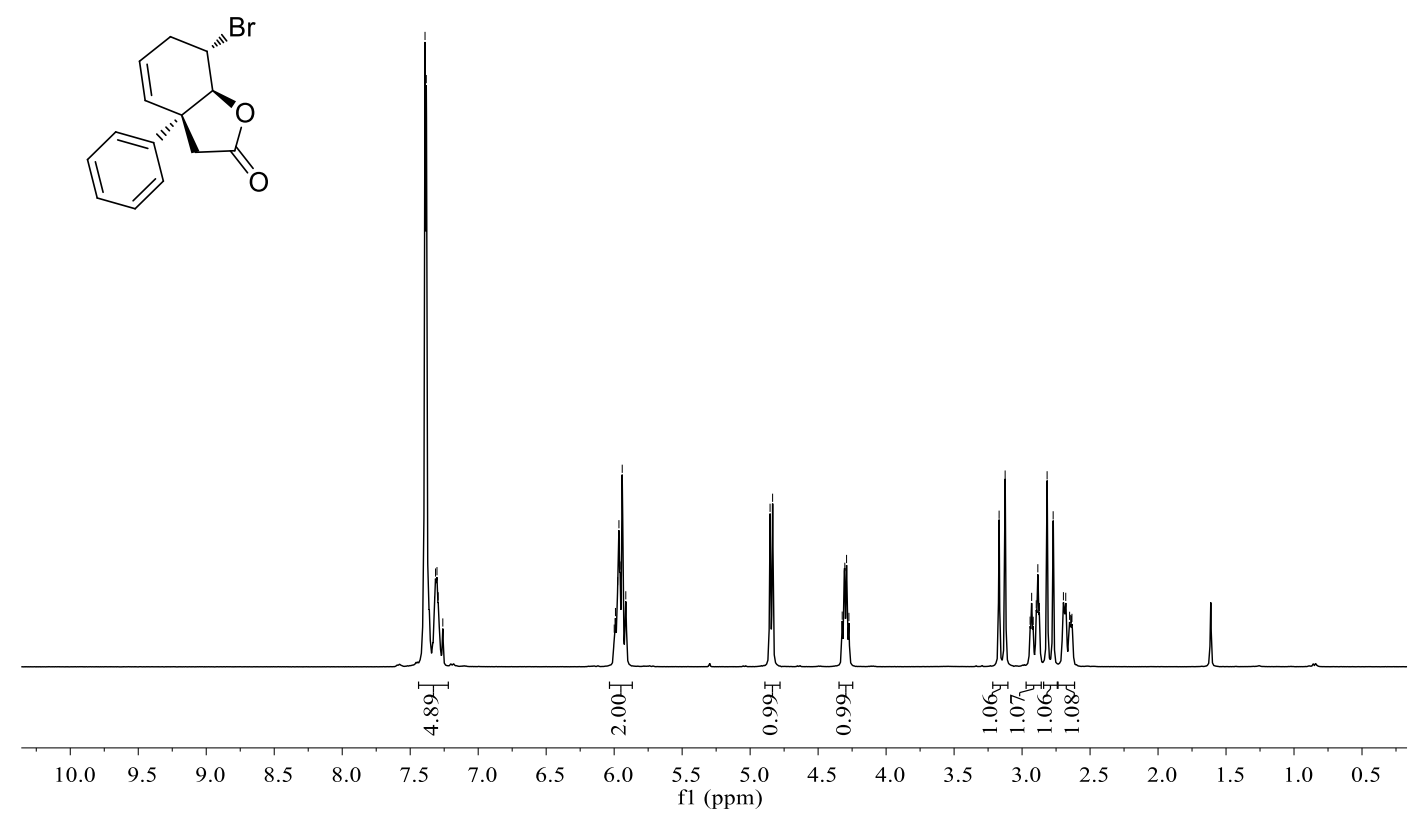

${ }^{13} \mathrm{C}$ NMR of $2 \mathrm{~s}\left(100 \mathrm{MHz}, \mathrm{CDCl}_{3}\right)$

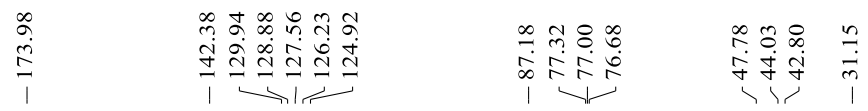

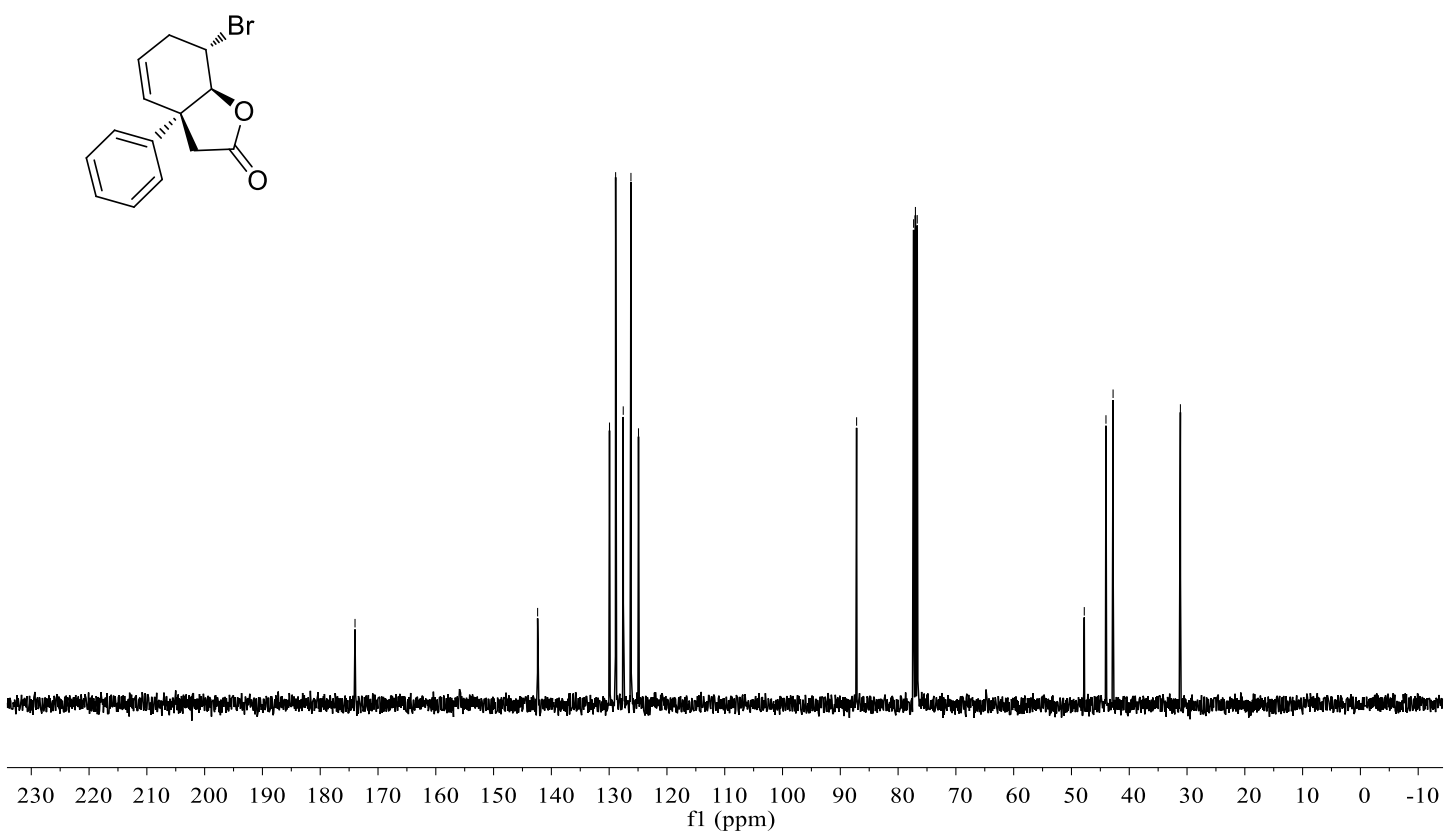


${ }^{1} \mathrm{H}$ NMR of $3\left(400 \mathrm{MHz}, \mathrm{CDCl}_{3}\right)$

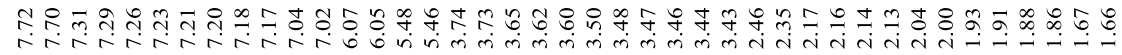<smiles>[AsH3-]N1CC[C@]2(c3ccccc3)C=CCCC12</smiles>

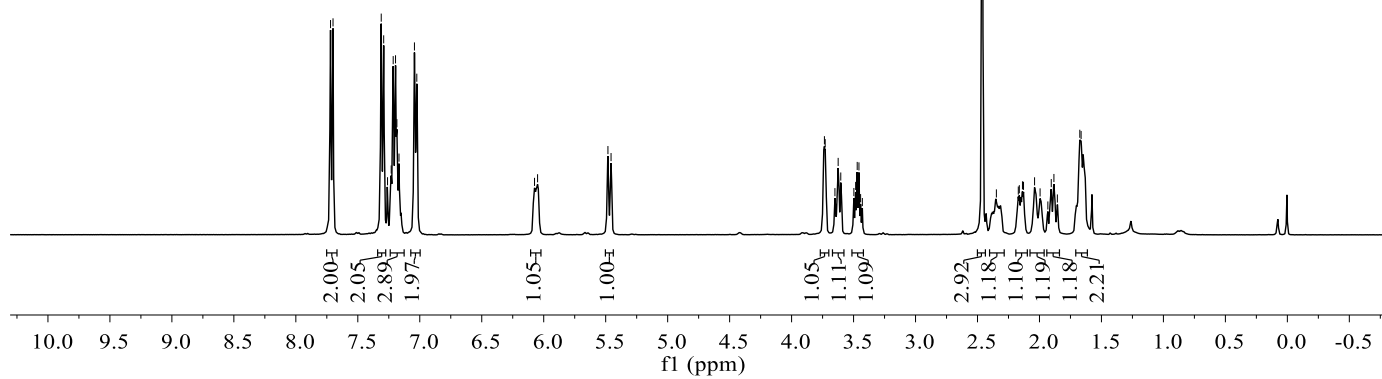

${ }^{13} \mathrm{C}$ NMR of $3\left(100 \mathrm{MHz}, \mathrm{CDCl}_{3}\right)$

ले ते

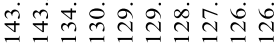

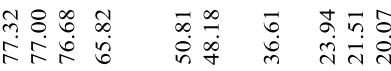

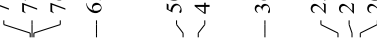<smiles>[AsH3-]N1CC[C@@]2(c3ccccc3)C=CCCC12</smiles>

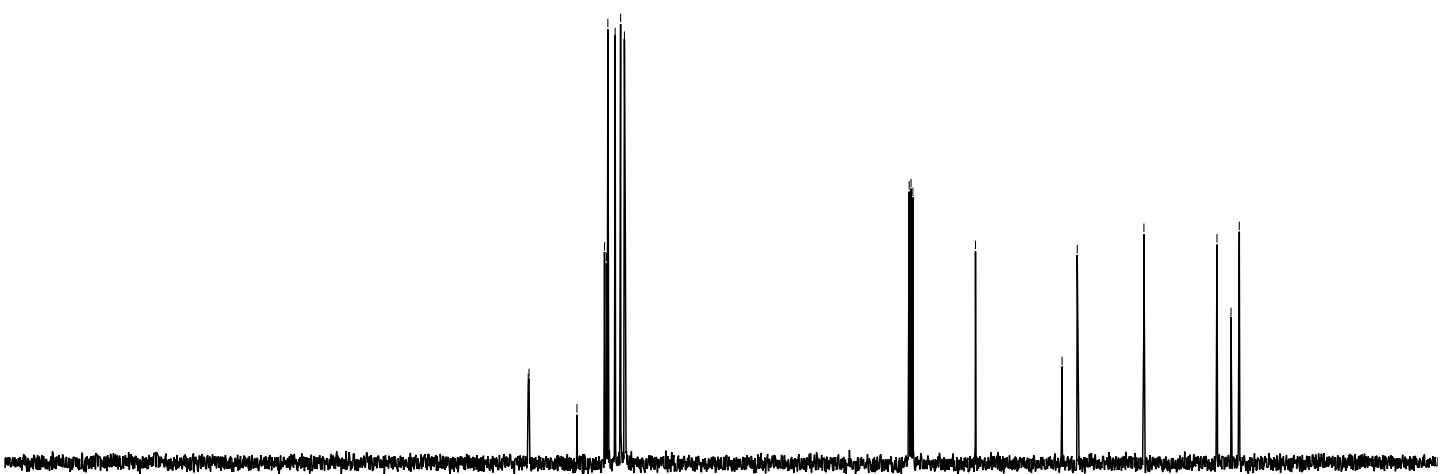

$\begin{array}{lllllllllllllllllllllllll}230 & 220 & 210 & 200 & 190 & 180 & 170 & 160 & 150 & 140 & 130 & 120 & 110 & 100 & 90 & 80 & 70 & 60 & 50 & 40 & 30 & 20 & 10 & 0 & -10\end{array}$ 
${ }^{1} \mathrm{H}$ NMR of $4\left(400 \mathrm{MHz}, \mathrm{CDCl}_{3}\right)$

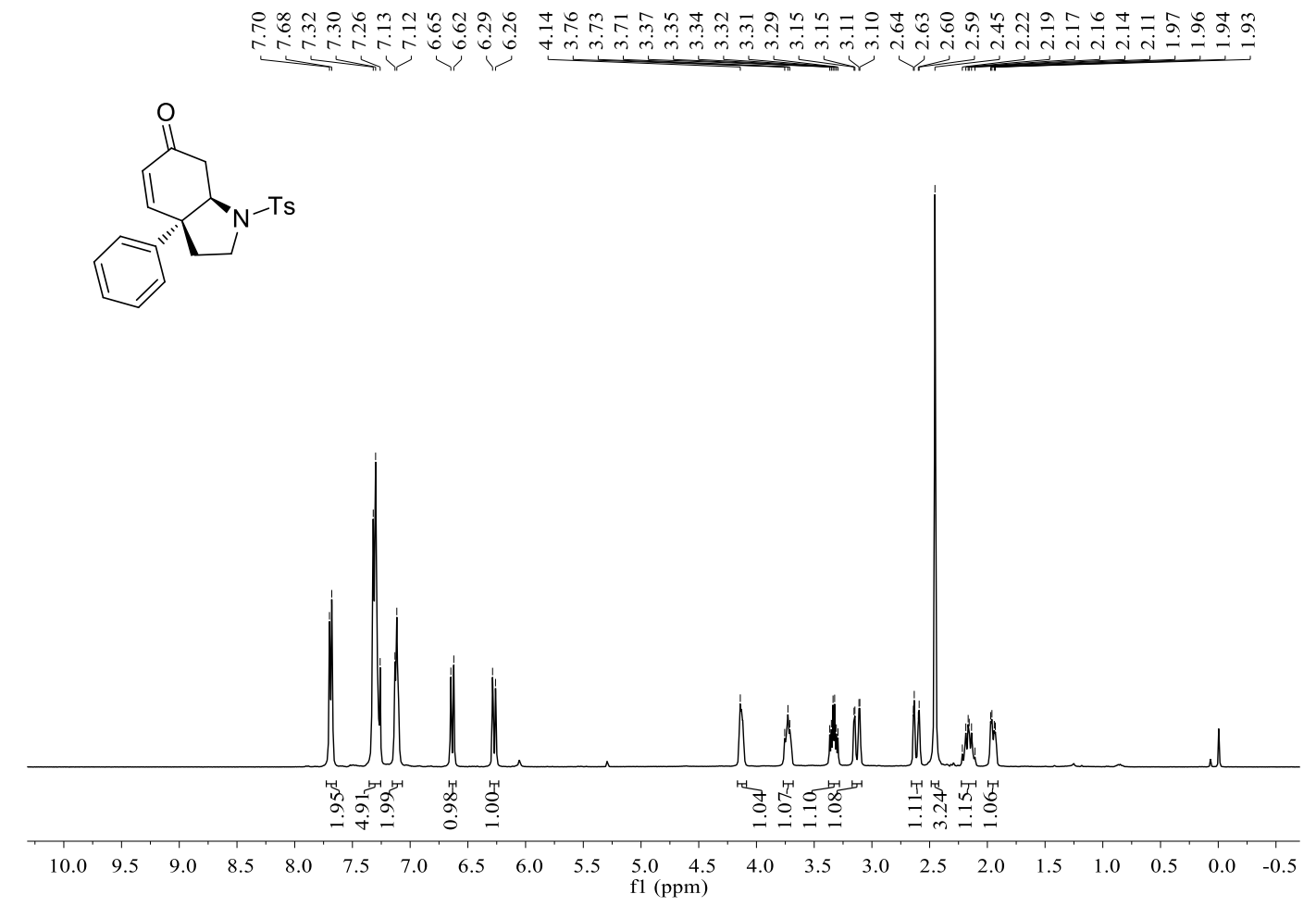

${ }^{13} \mathrm{C}$ NMR of 4 (100 MHz, CDCl3)

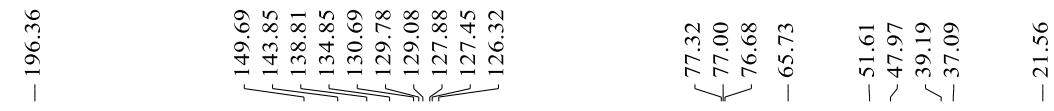

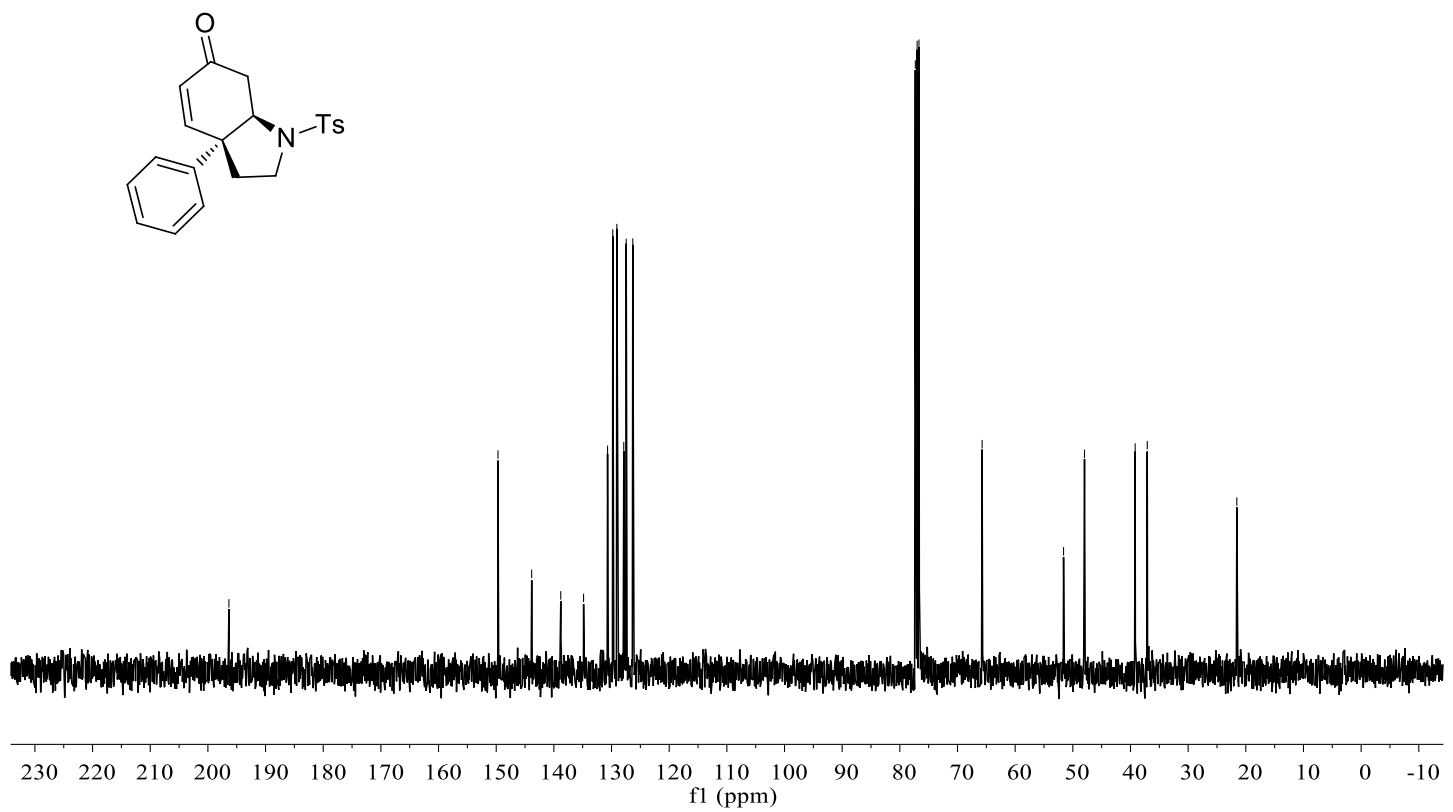


${ }^{1} \mathrm{H}$ NMR of 5 (400 MHz, CDCl 3$)$

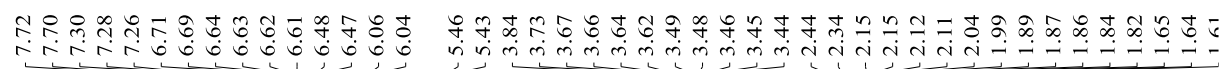<smiles>COc1ccc(C23C=CCCC2N([13F])CC3)cc1OC</smiles>

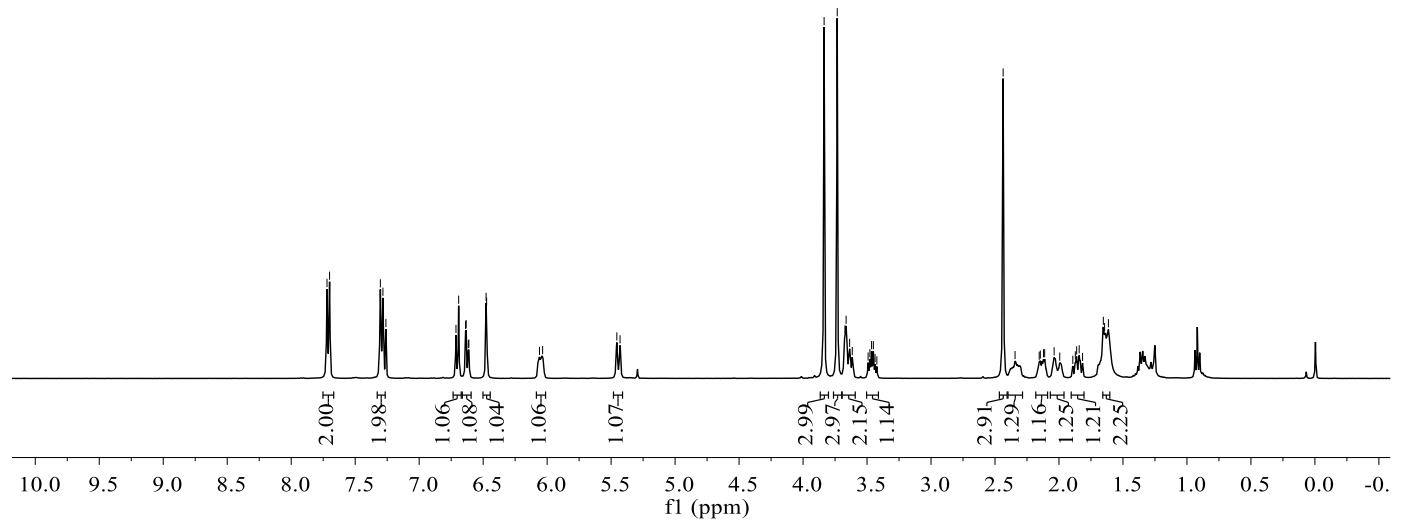

${ }^{13} \mathrm{C} \mathrm{NMR}$ of $5\left(100 \mathrm{MHz}, \mathrm{CDCl}_{3}\right)$

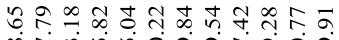

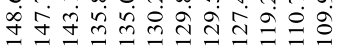

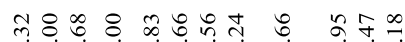

송<smiles>COc1ccc(C23C=CCCC2N([135I])CC3)cc1OC</smiles>

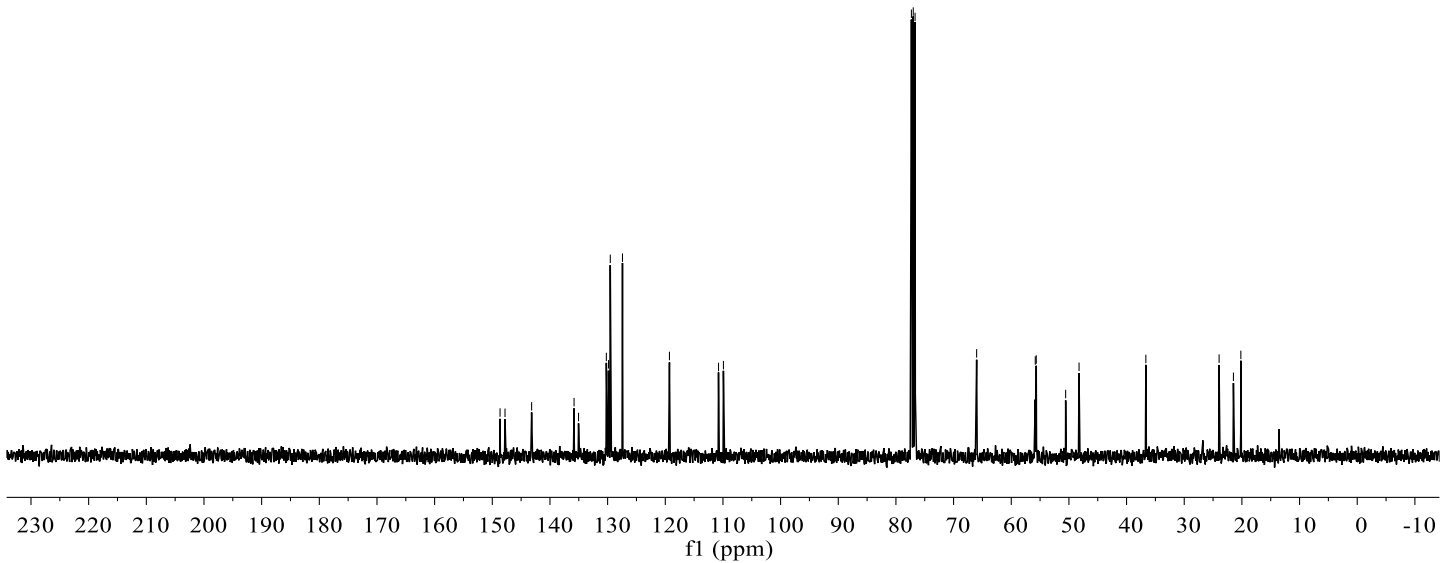


${ }^{1} \mathrm{H}$ NMR of $6\left(400 \mathrm{MHz}, \mathrm{CDCl}_{3}\right)$

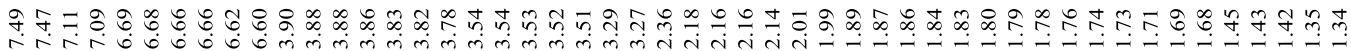<smiles>[3H]N1CC[C@@]2(c3ccc(OC)c(OC)c3)CCCCC12</smiles>

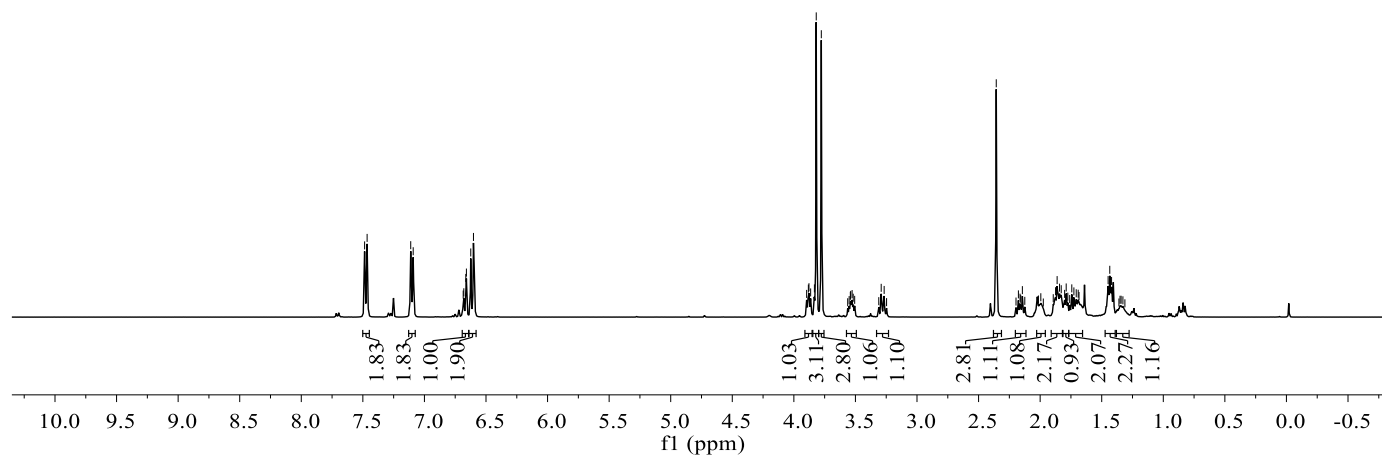

${ }^{13} \mathrm{C}$ NMR of $6\left(100 \mathrm{MHz}, \mathrm{CDCl}_{3}\right)$

nิํำ

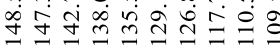

귱ㅇㅇ

人, 1,

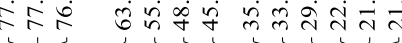<smiles>COc1ccc([C@]23CCCC[C@H]2N([13F])CC3)cc1OC</smiles>

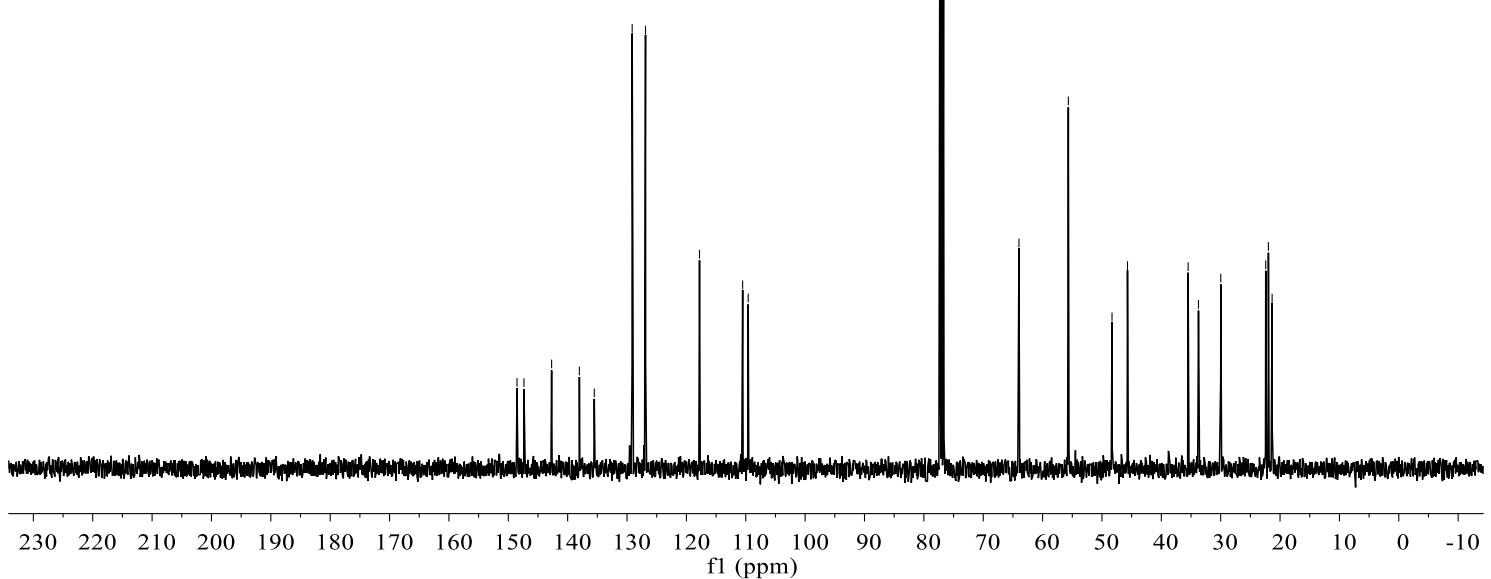


${ }^{1} \mathrm{H}$ NMR of 8: (+)-Mesembrane (400 $\mathrm{MHz}^{\mathrm{CDCl}} 3$ )

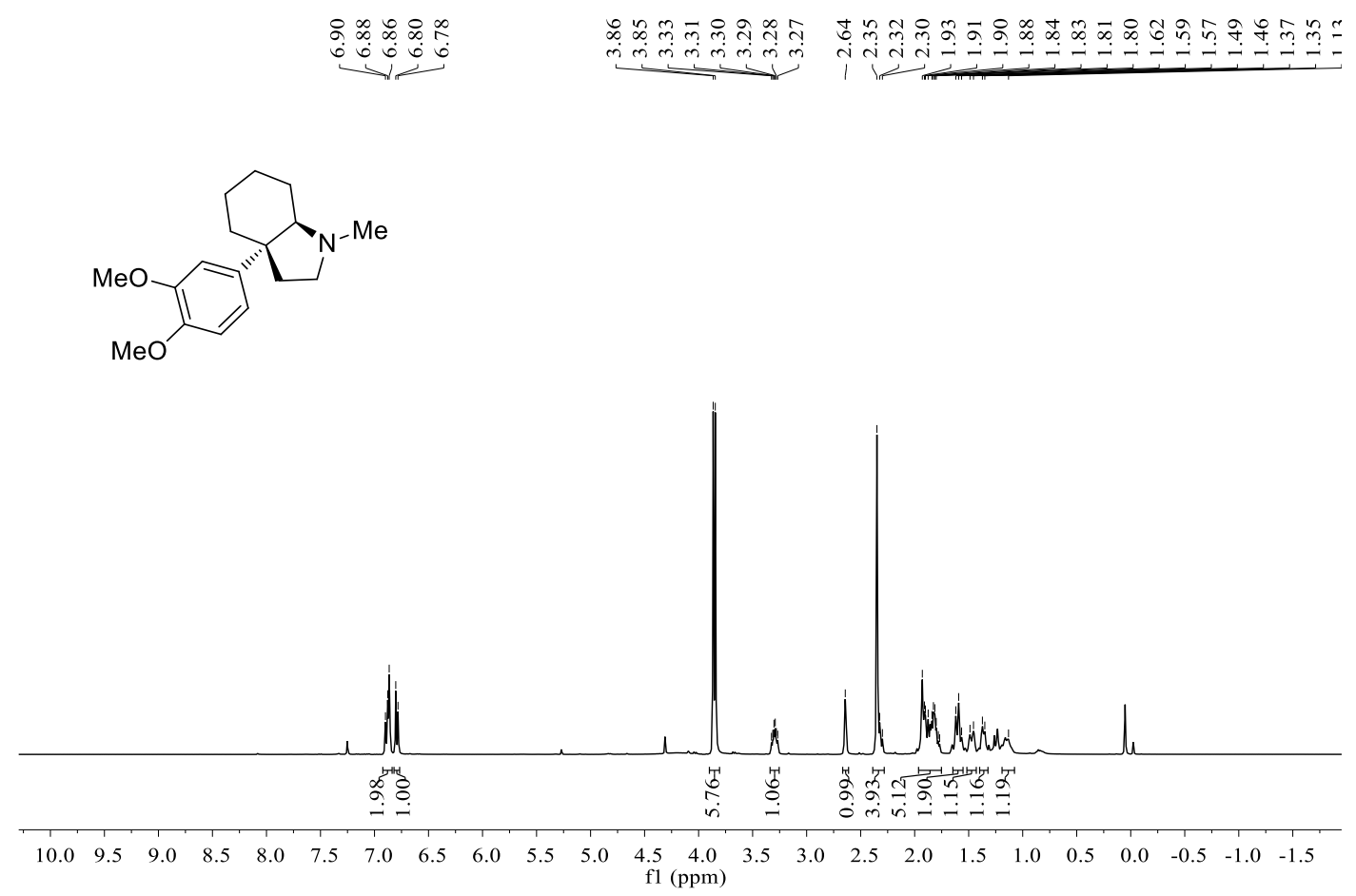

${ }^{13} \mathrm{C}$ NMR of 8: (+)-Mesembrane (100 $\left.\mathrm{MHz}, \mathrm{CDCl}_{3}\right)$
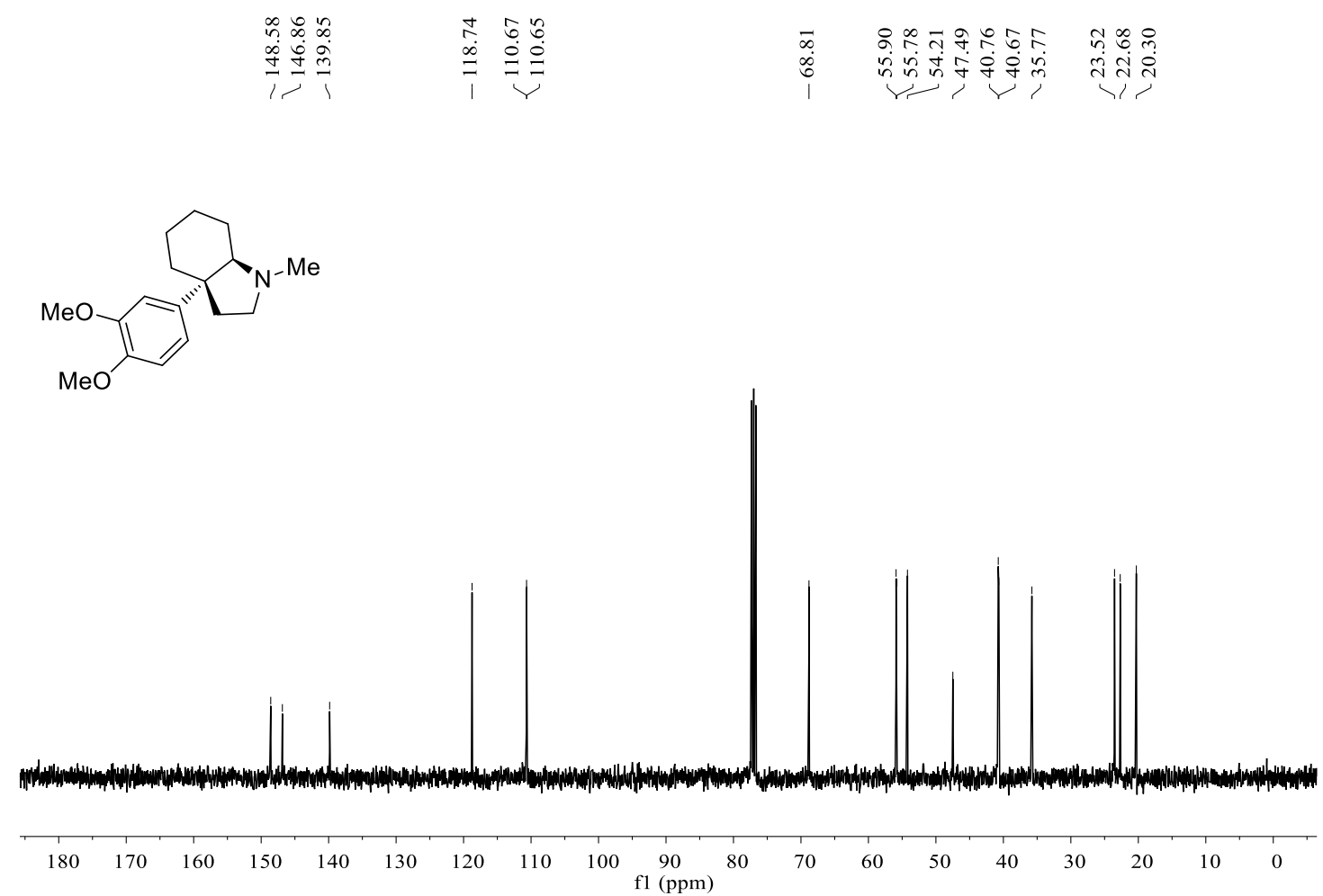


\section{HPLC Reports}

HPLC Analysis of 2a
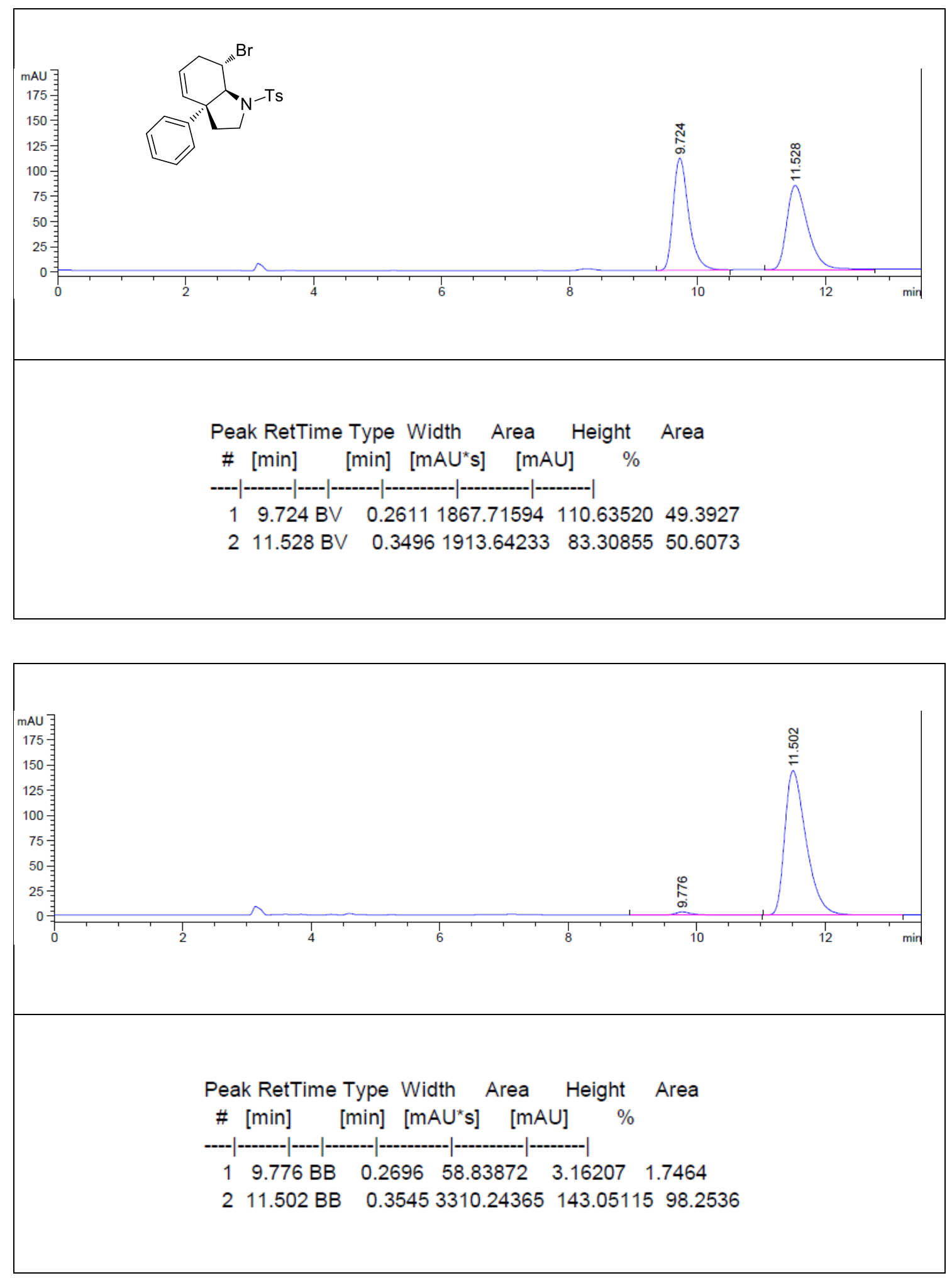
HPLC Analysis of 2a'
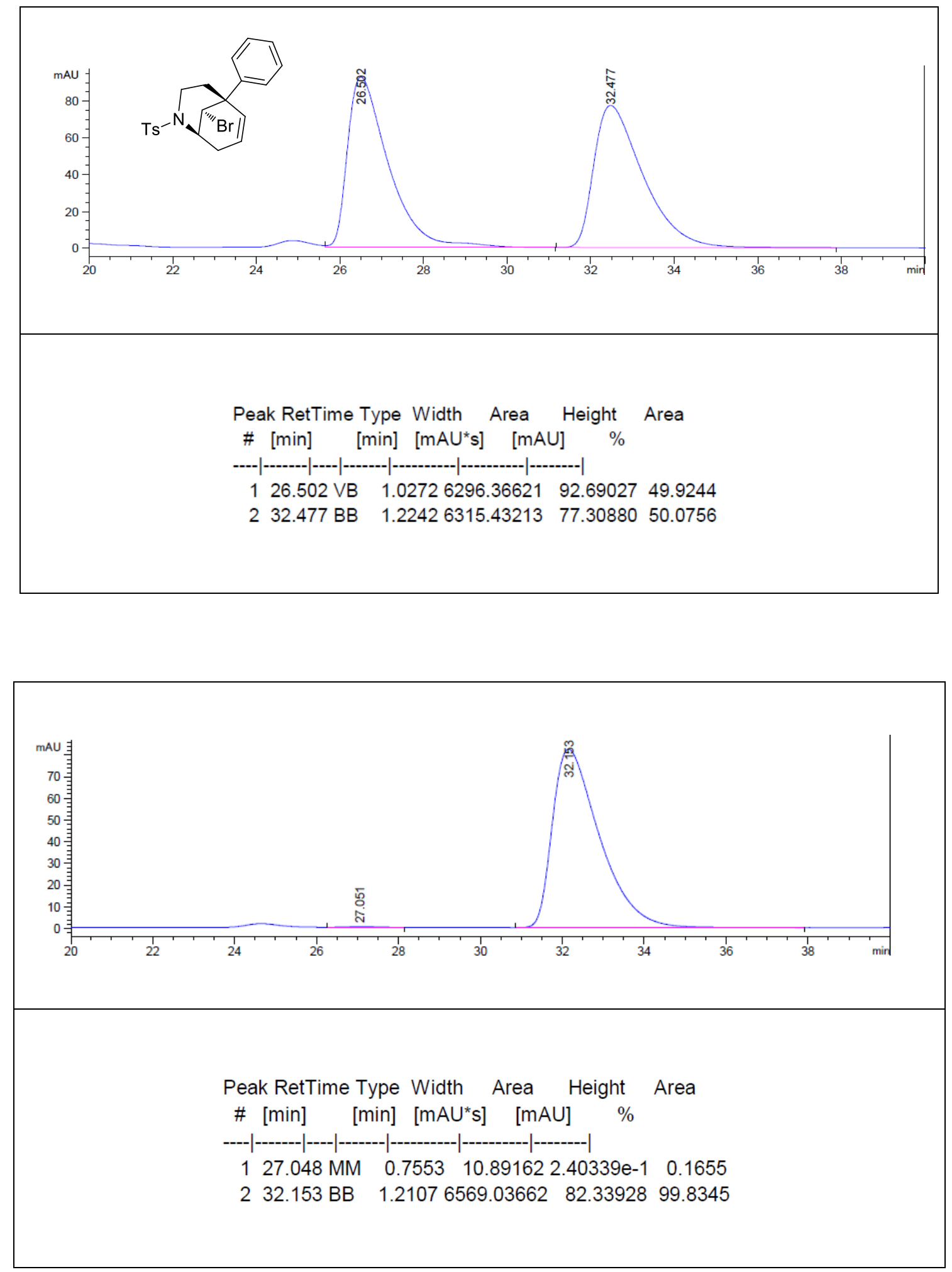
HPLC Analysis of $2 b$
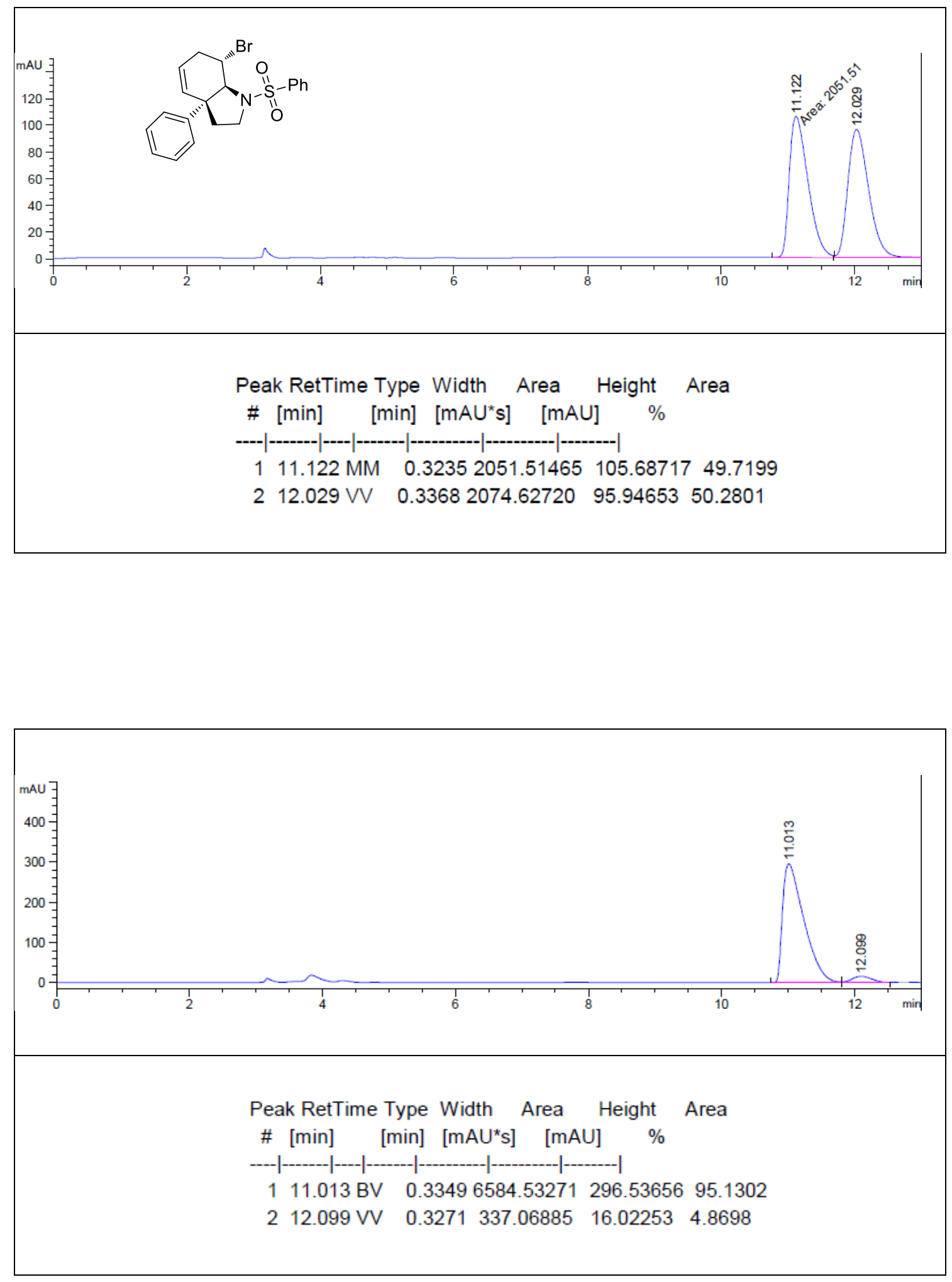
HPLC Analysis of 2c
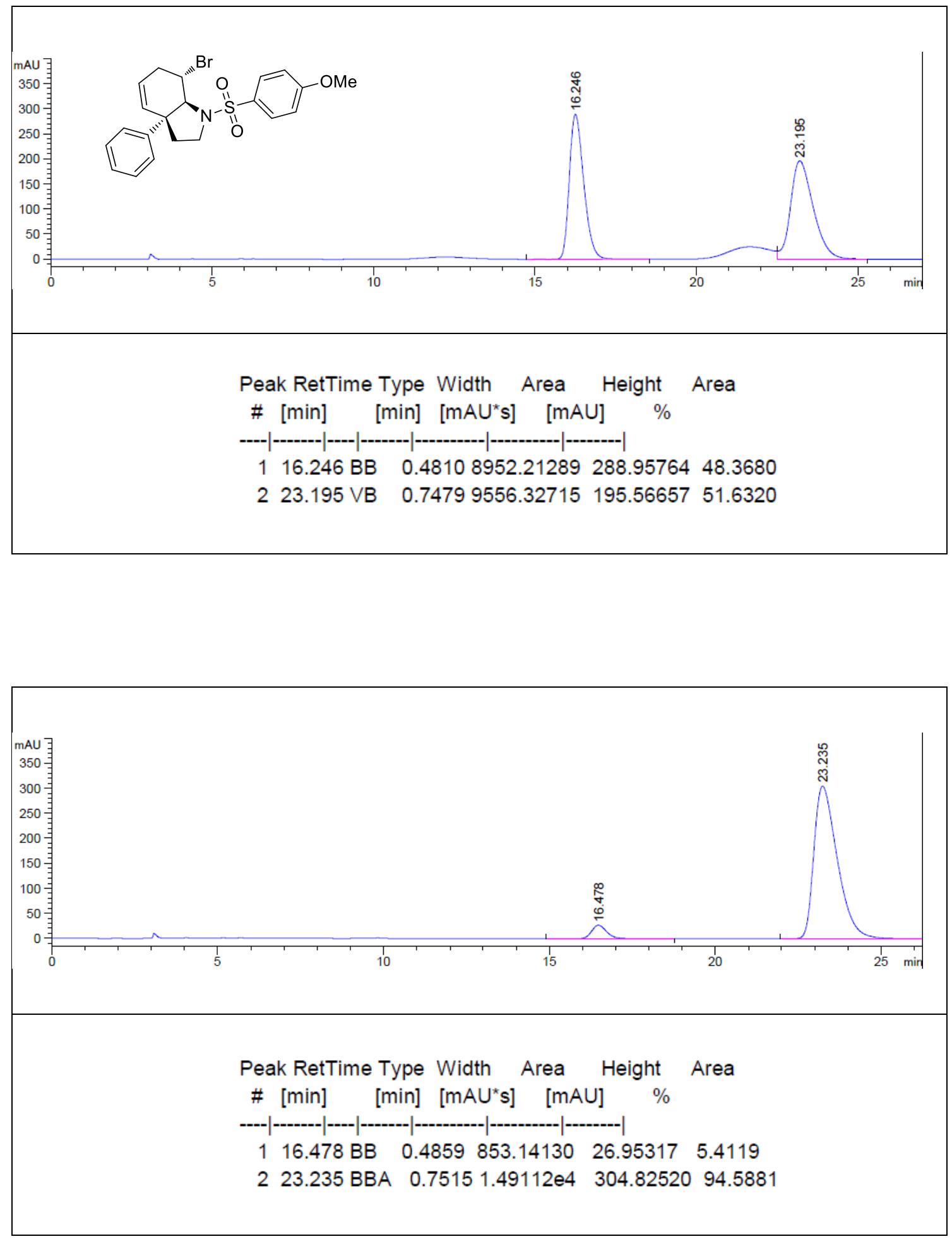

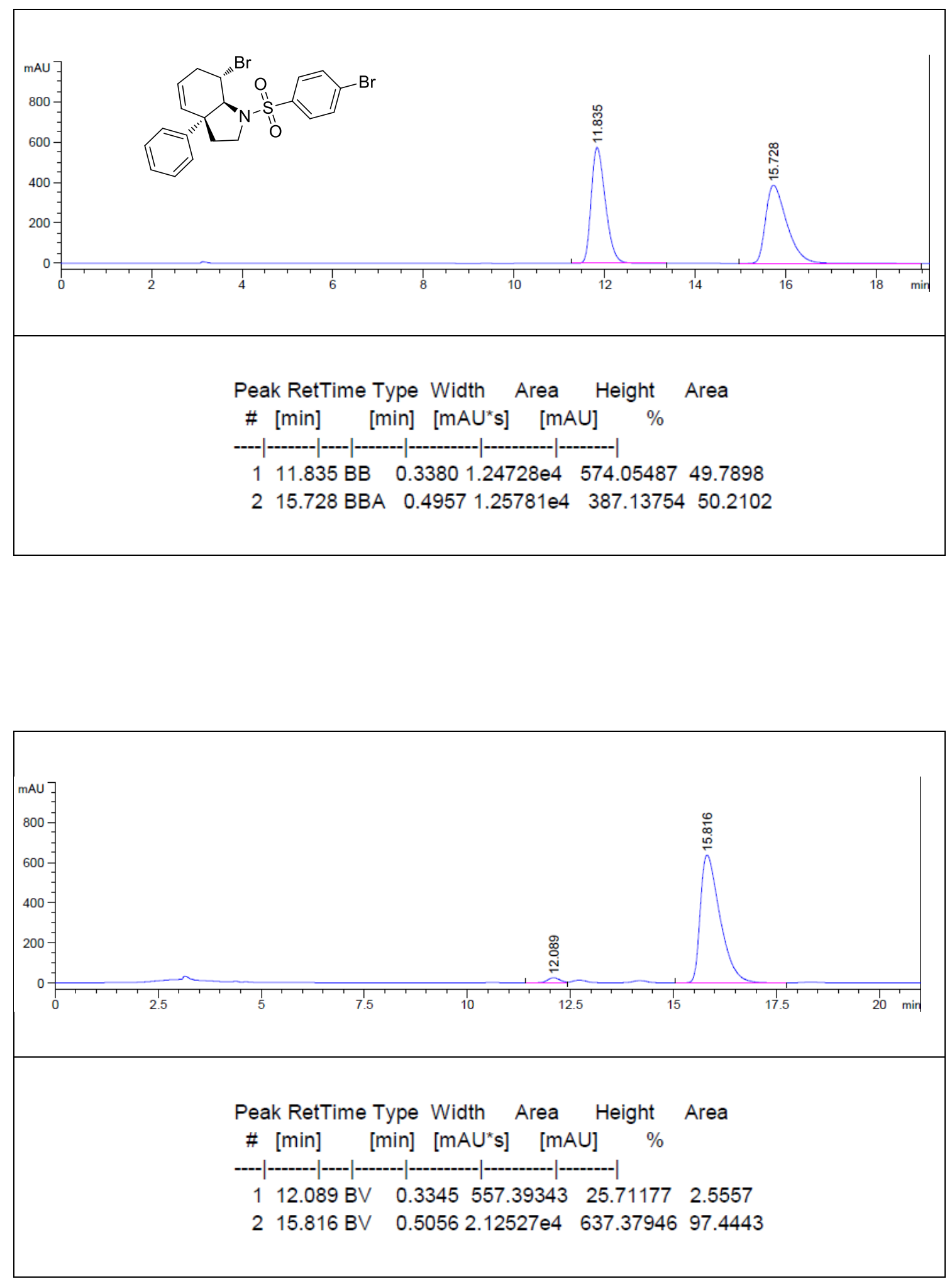
HPLC Analysis of $2 e$
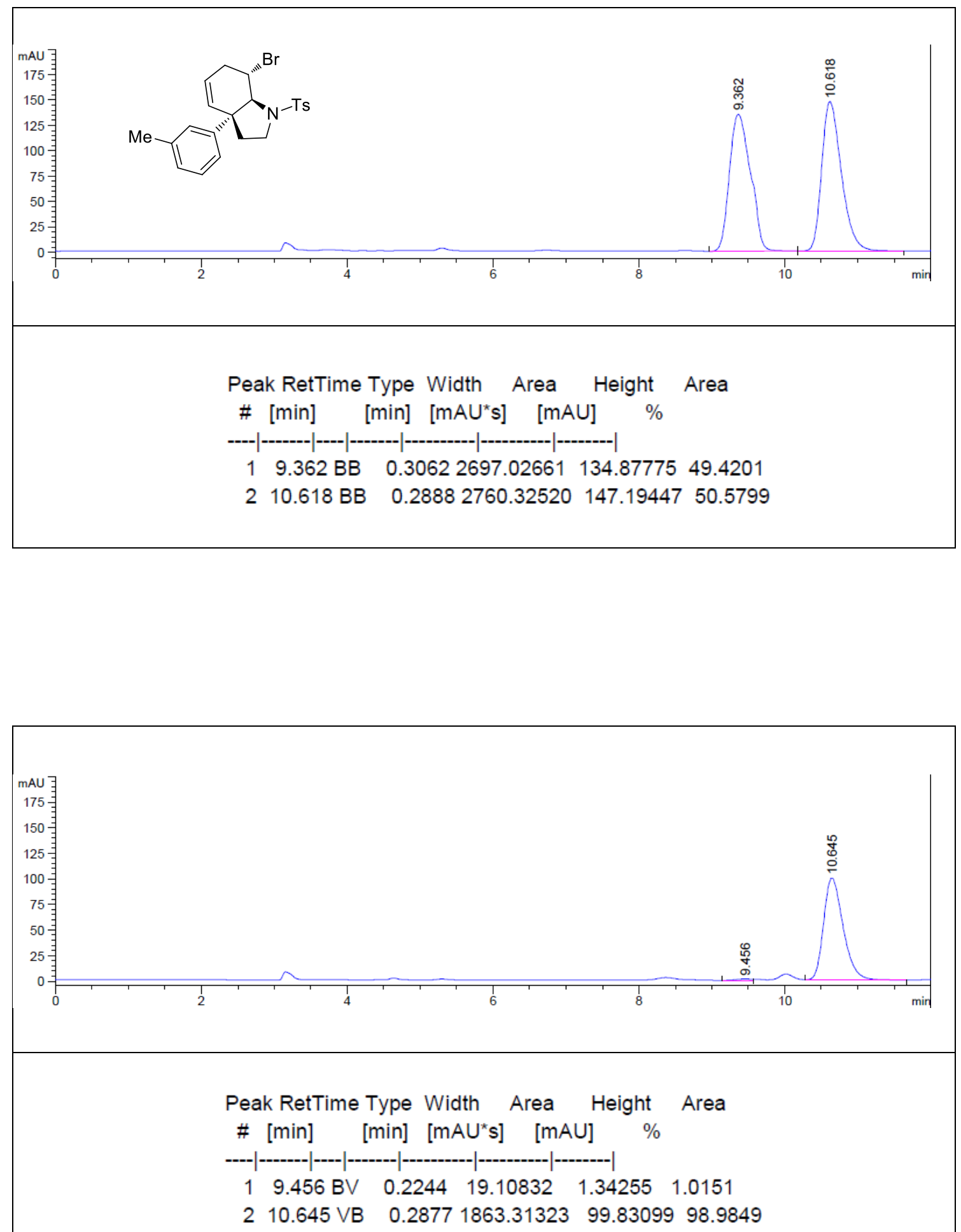

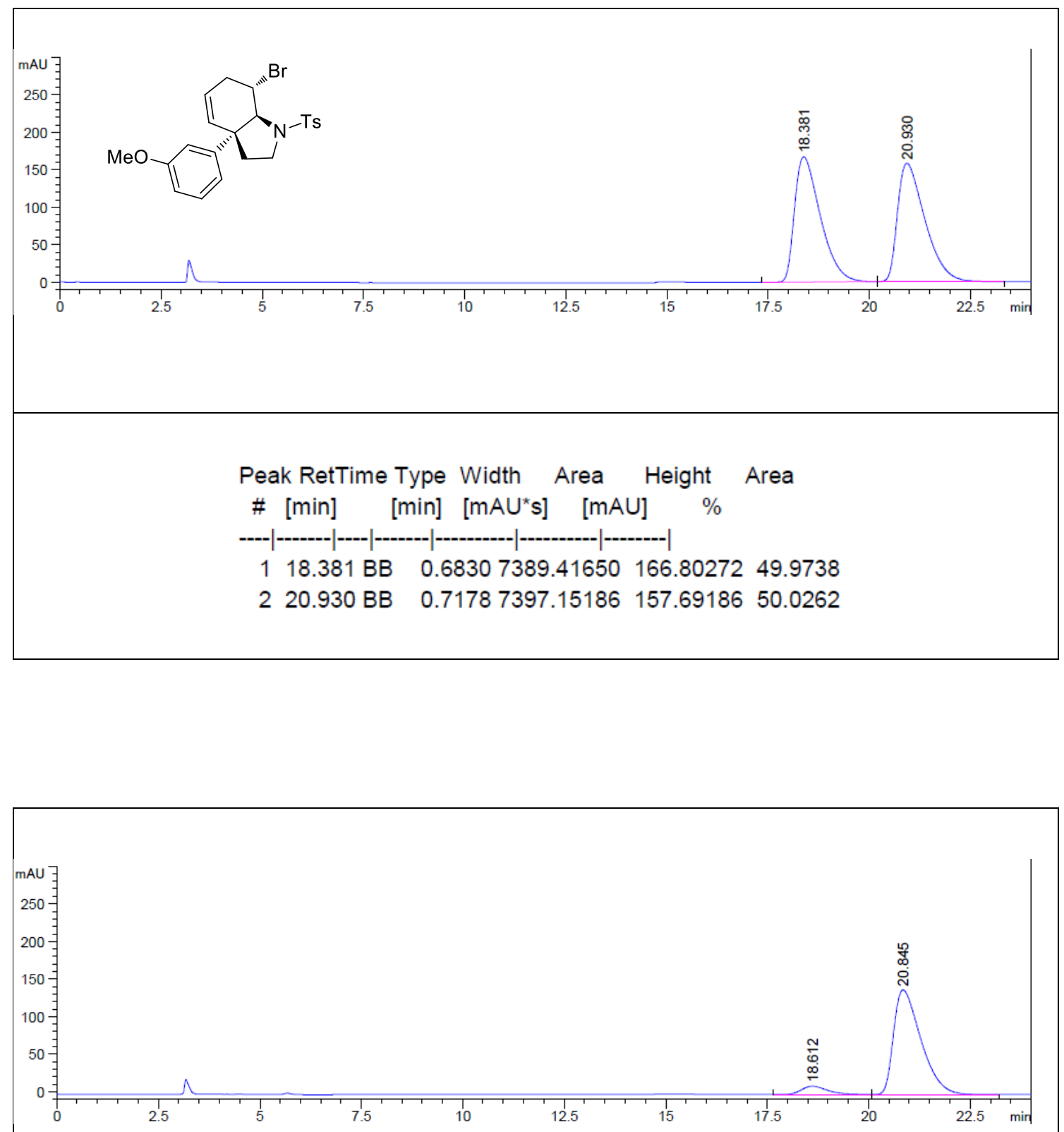

Peak RetTime Type Width Area Height Area \# [min] [min] [mAU*s] [mAU] $\%$

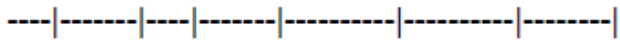

$\begin{array}{llllll}1 & 18.612 \mathrm{BB} & 0.6724 & 502.24887 & 11.18837 & 7.1532\end{array}$

220.845 BB $\quad 0.72256519 .06934 \quad 139.26468 \quad 92.8468$ 
HPLC Analysis of $2 \mathrm{~g}$
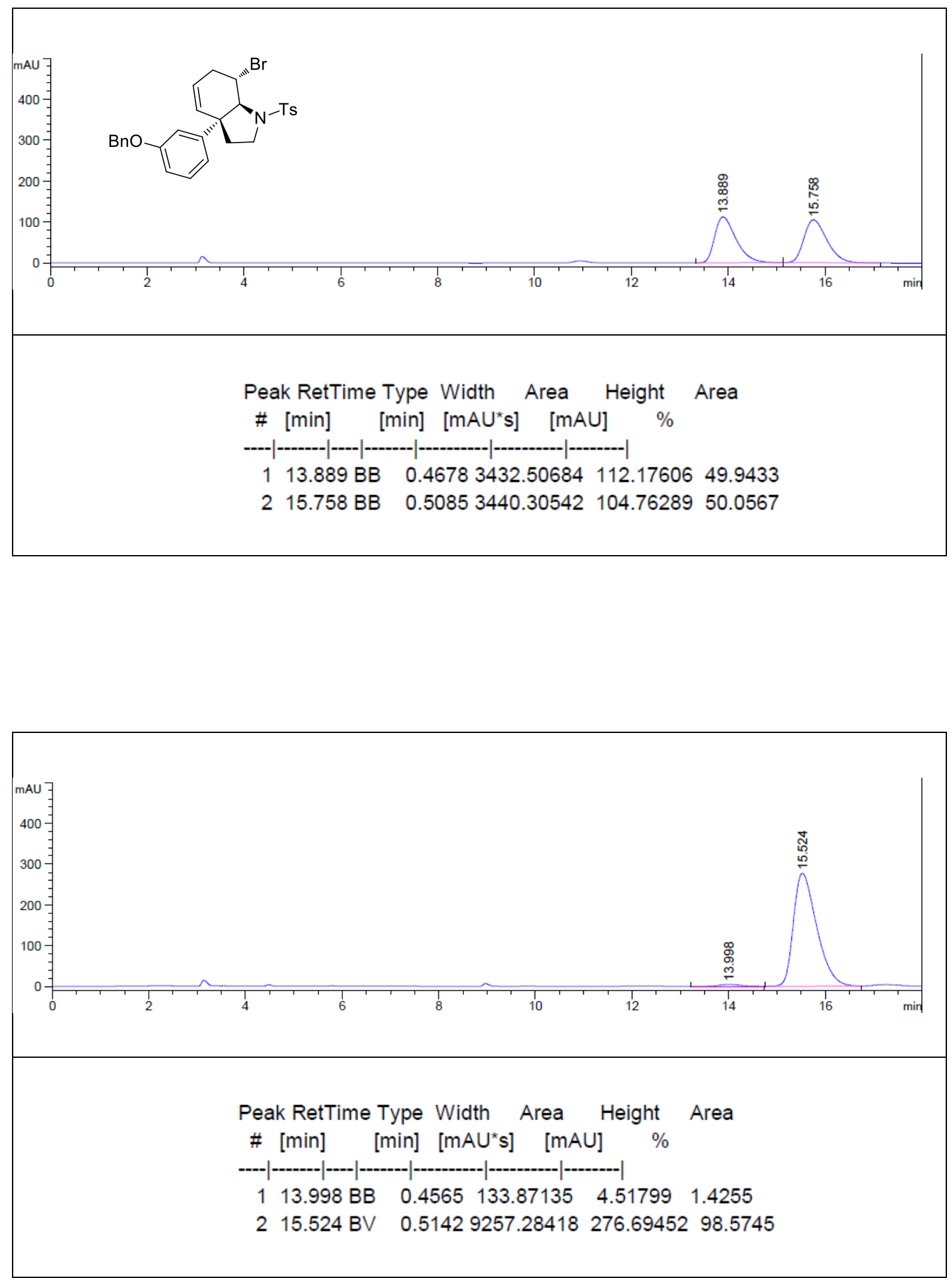
HPLC Analysis of $2 \mathrm{~h}$
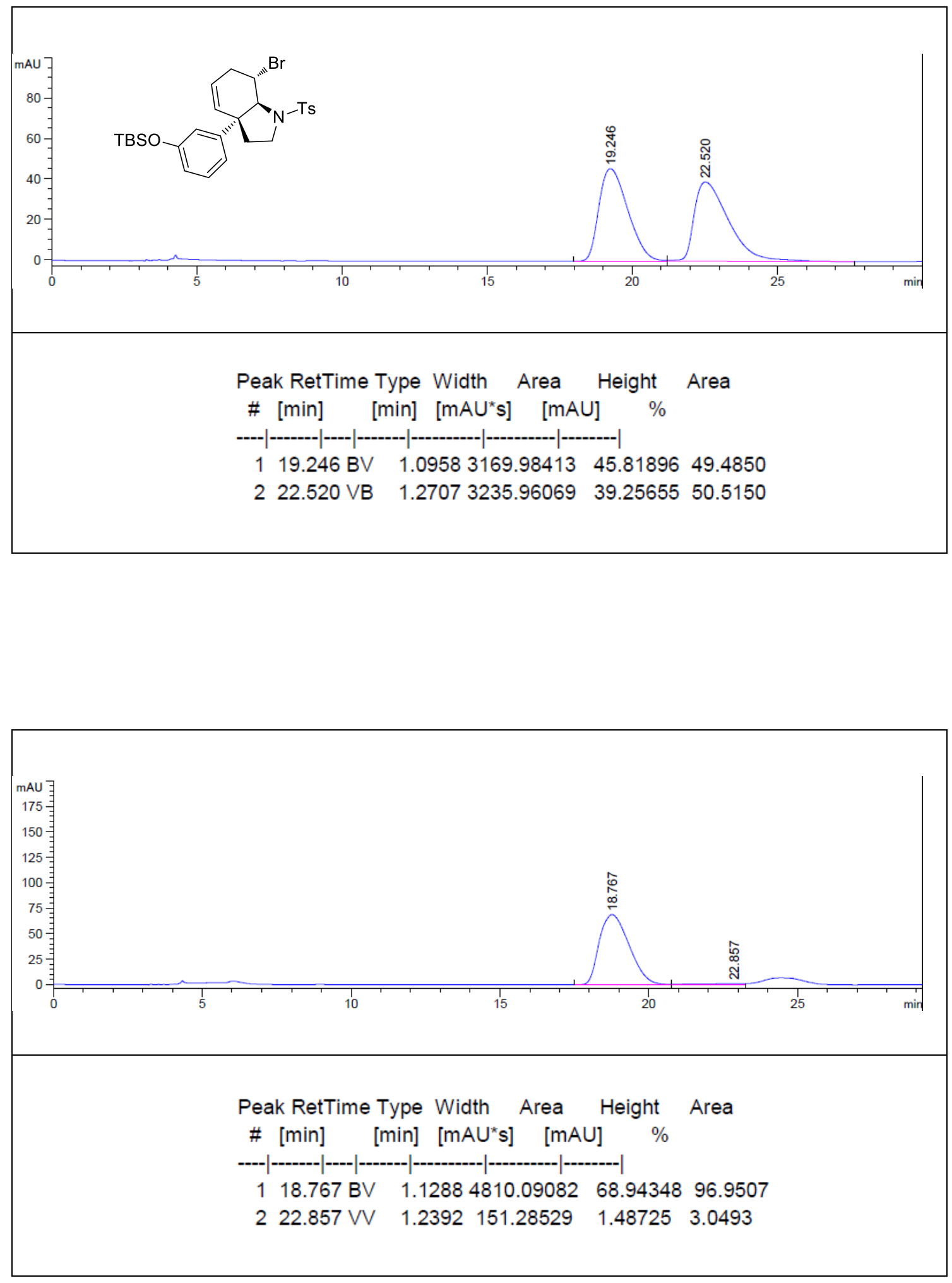
HPLC Analysis of $2 \mathrm{i}$
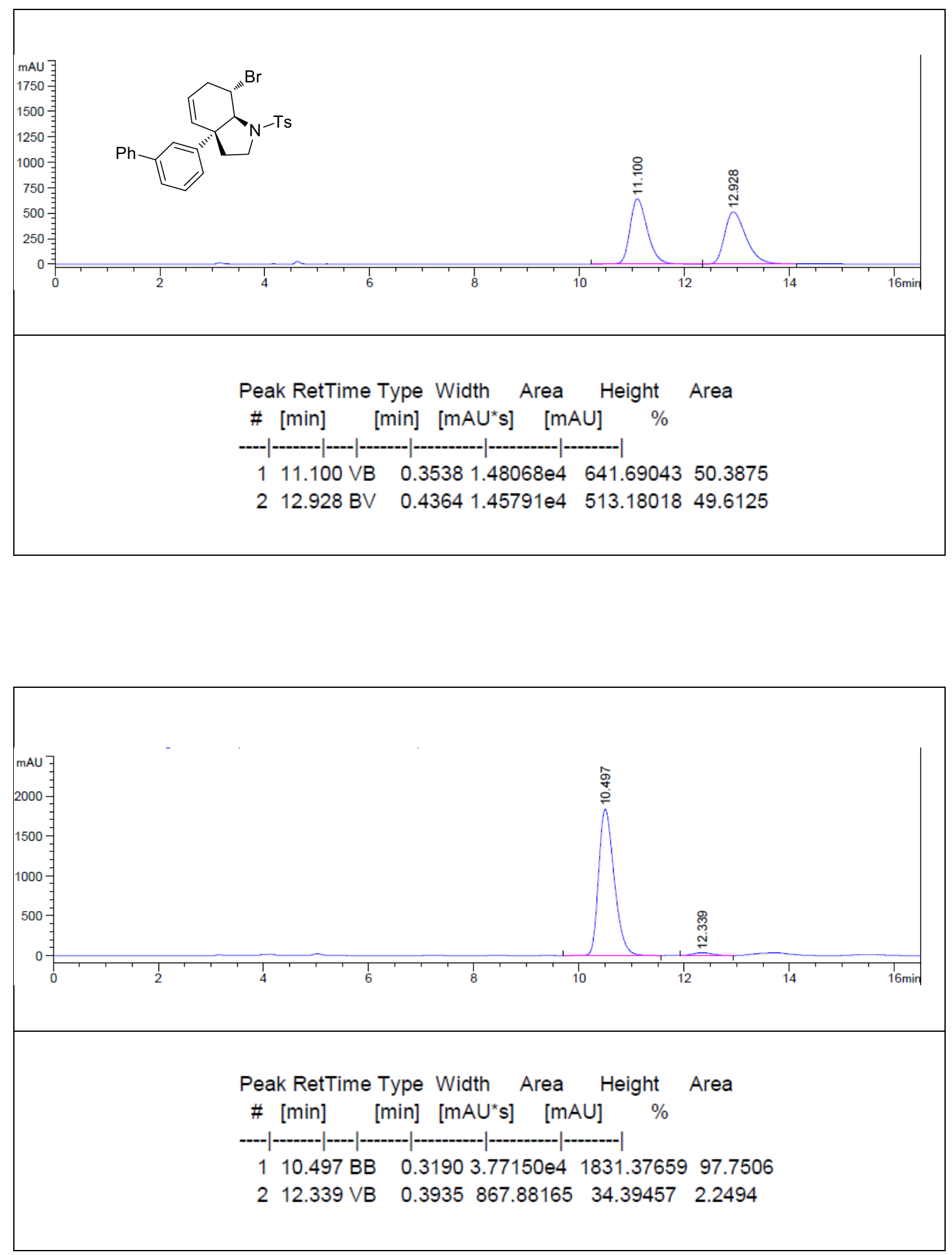
HPLC Analysis of $2 \mathrm{j}$
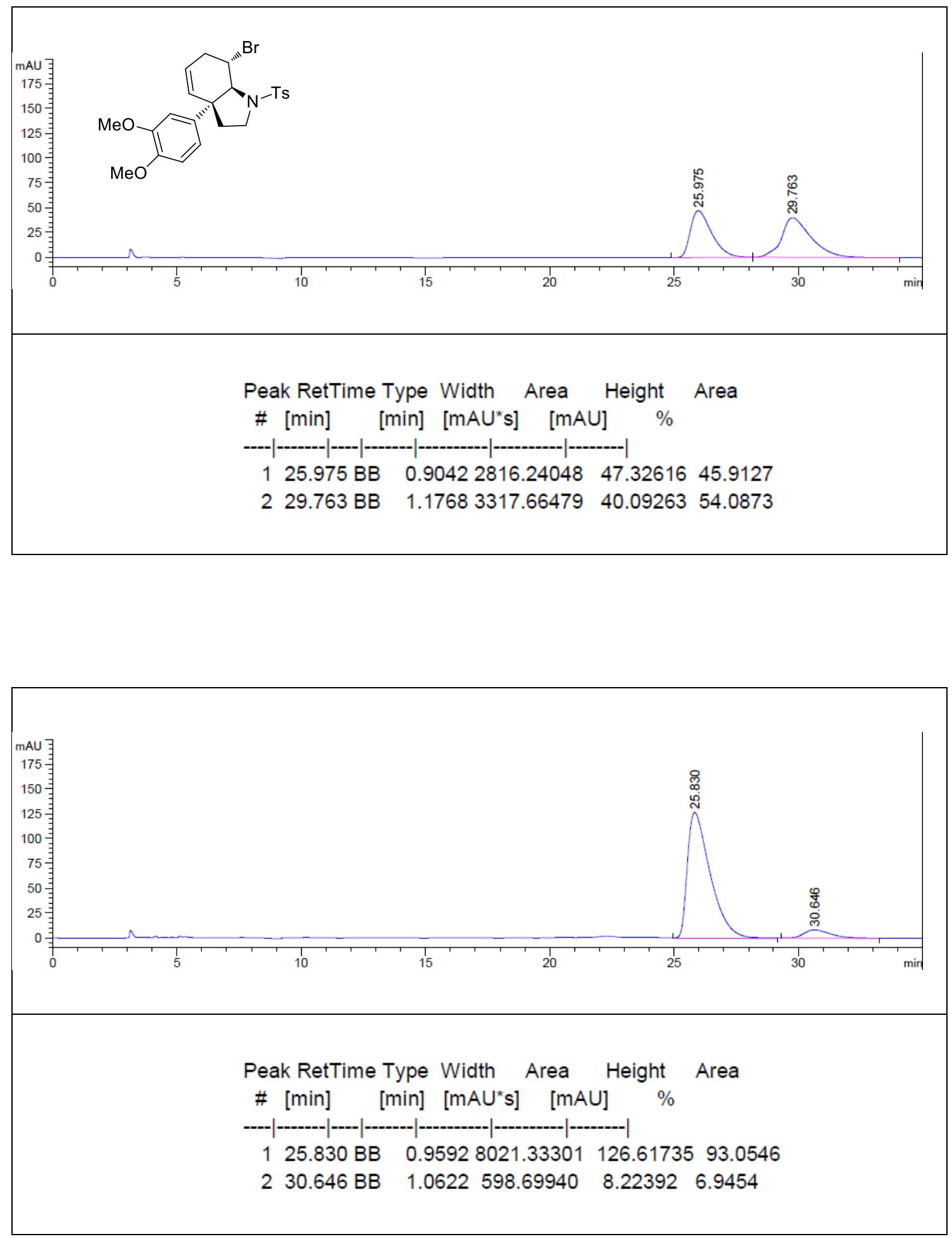
HPLC Analysis of $2 \mathrm{k}$
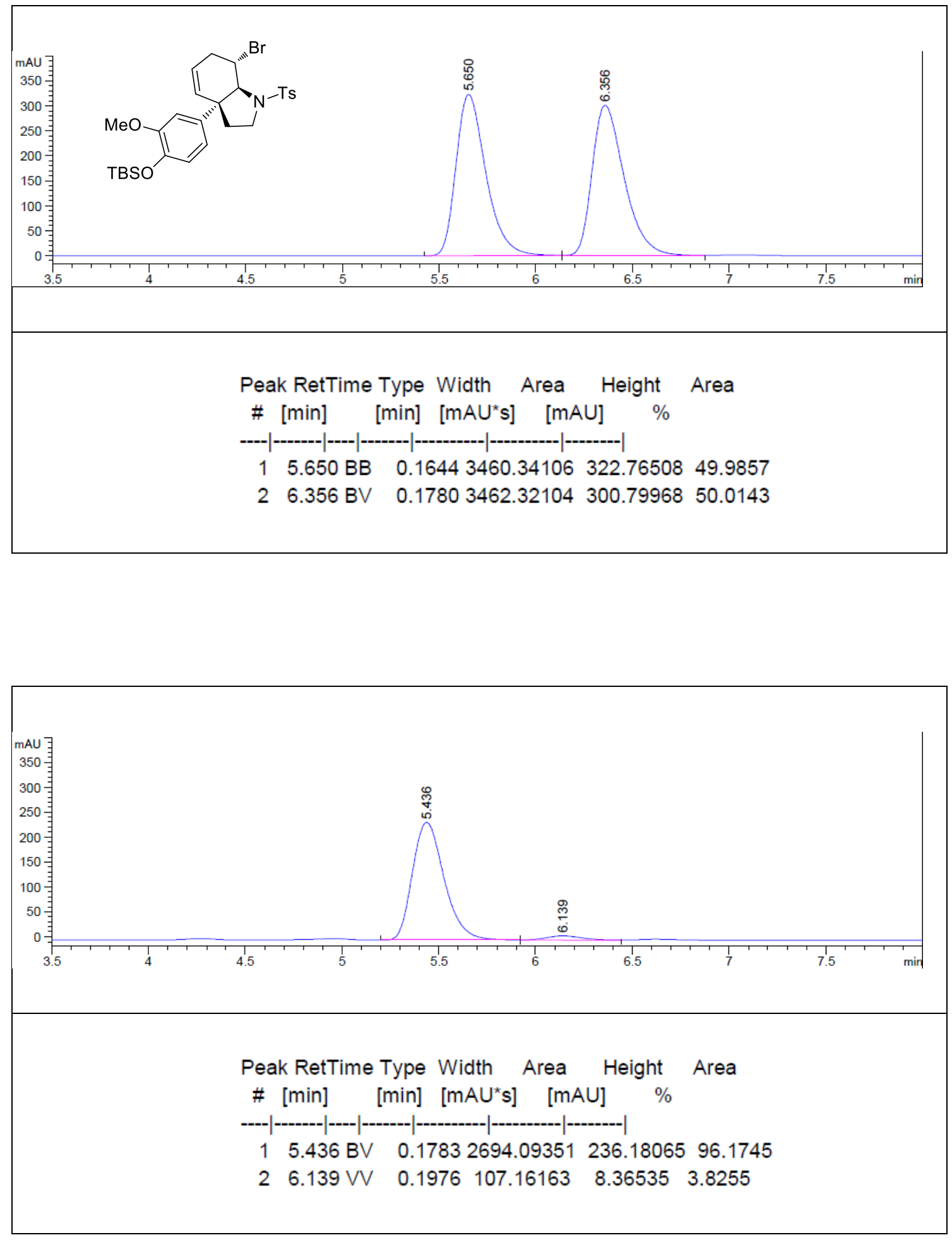
HPLC Analysis of 21
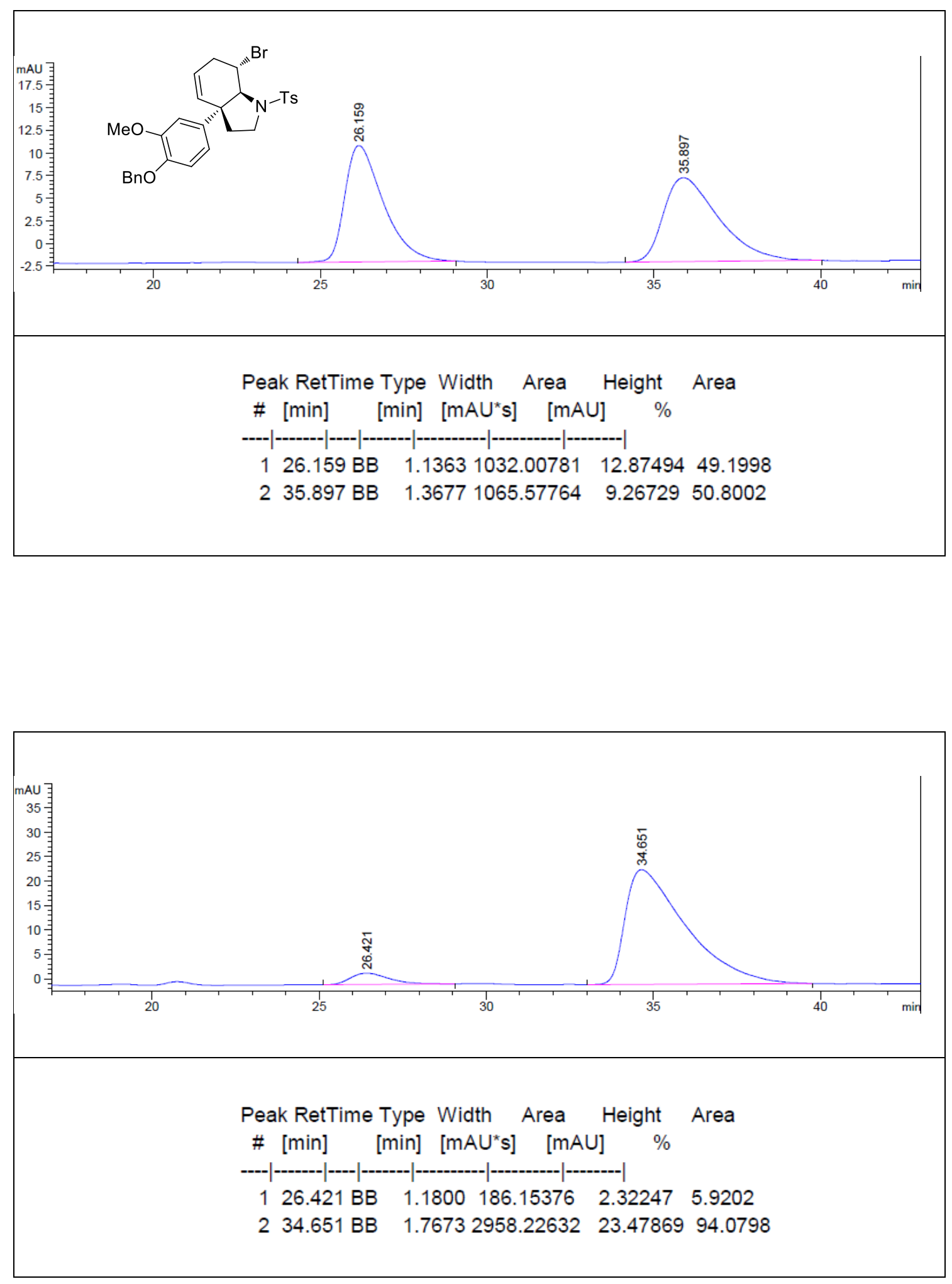
HPLC Analysis of $2 \mathrm{~m}$
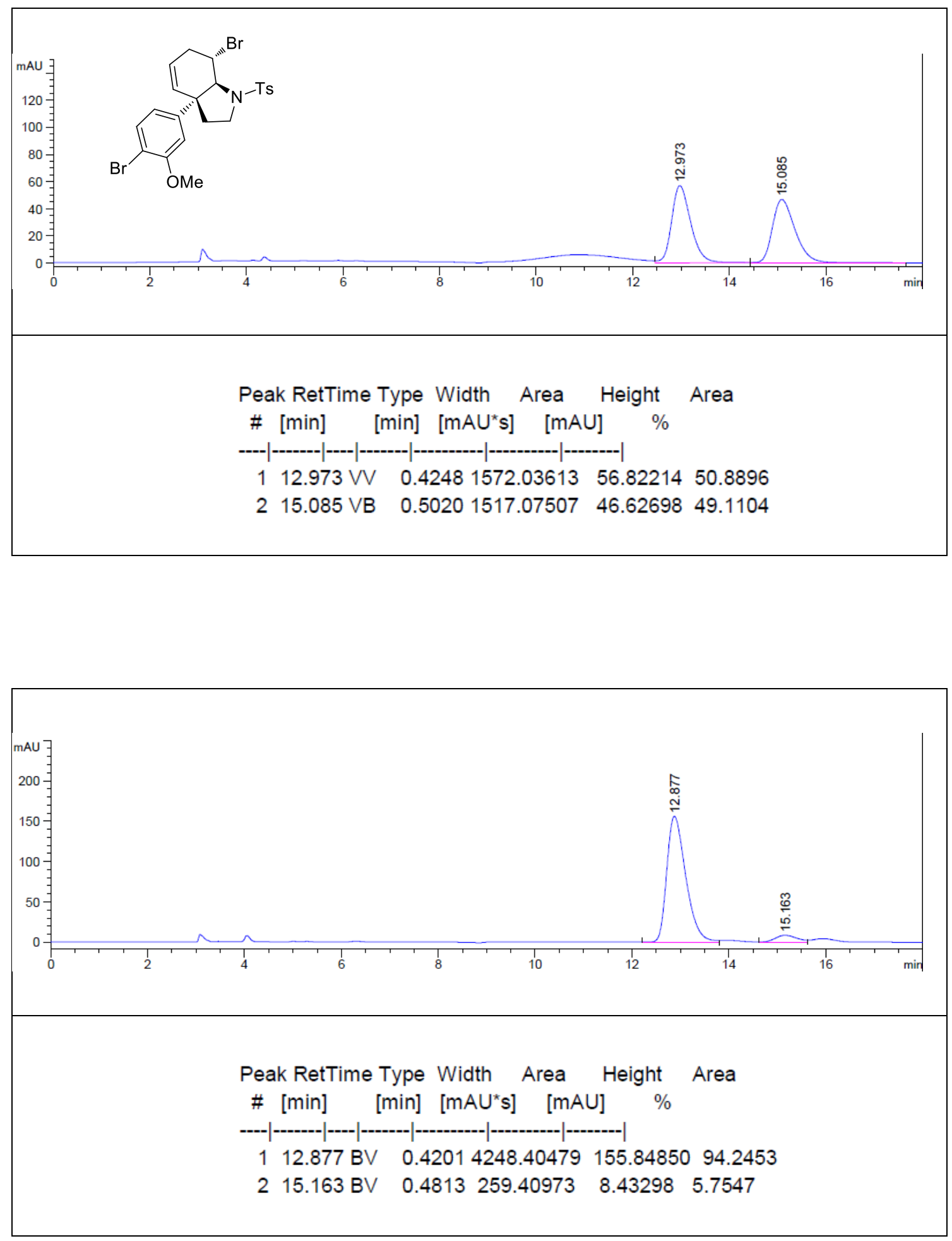
HPLC Analysis of 2n
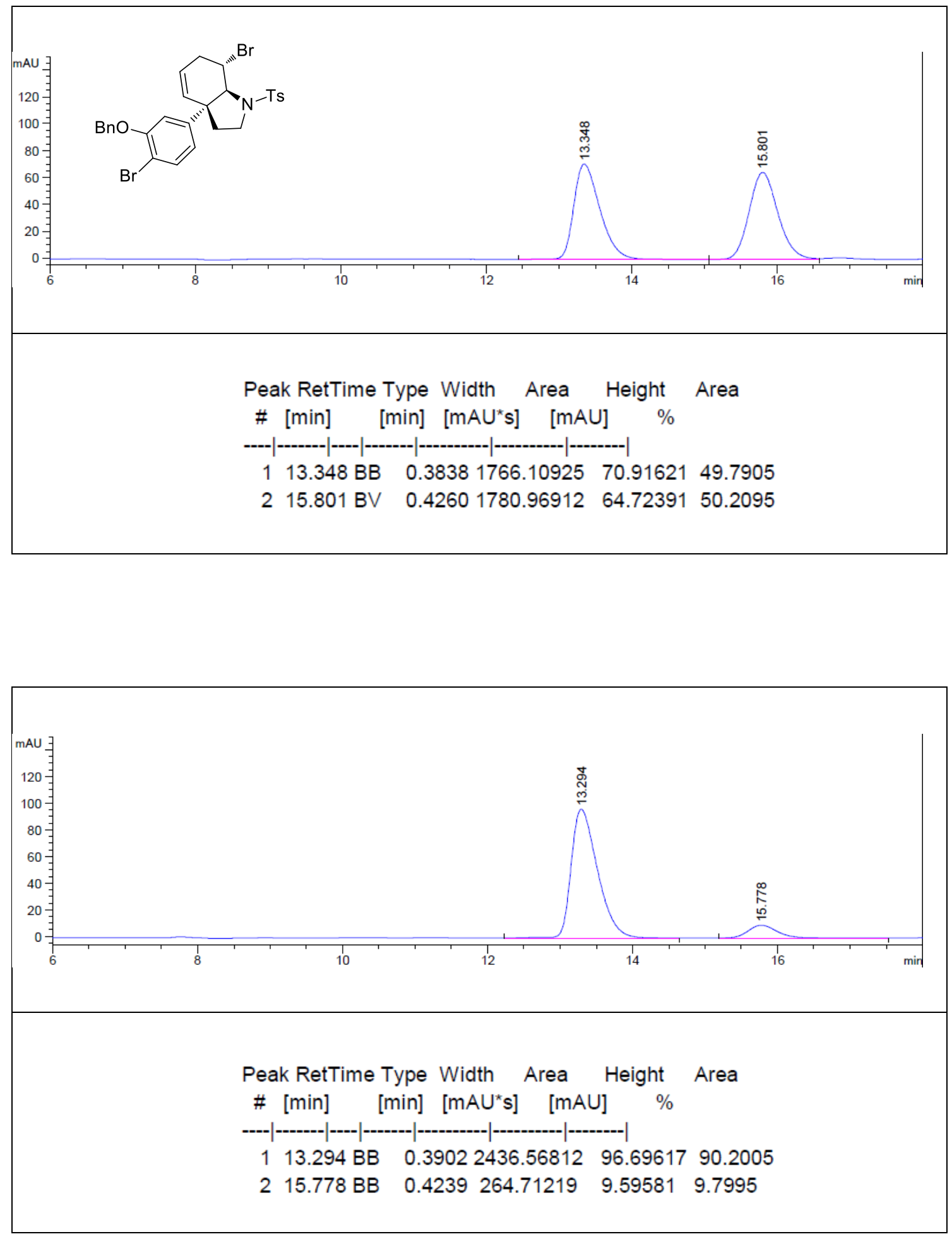
HPLC Analysis of 20
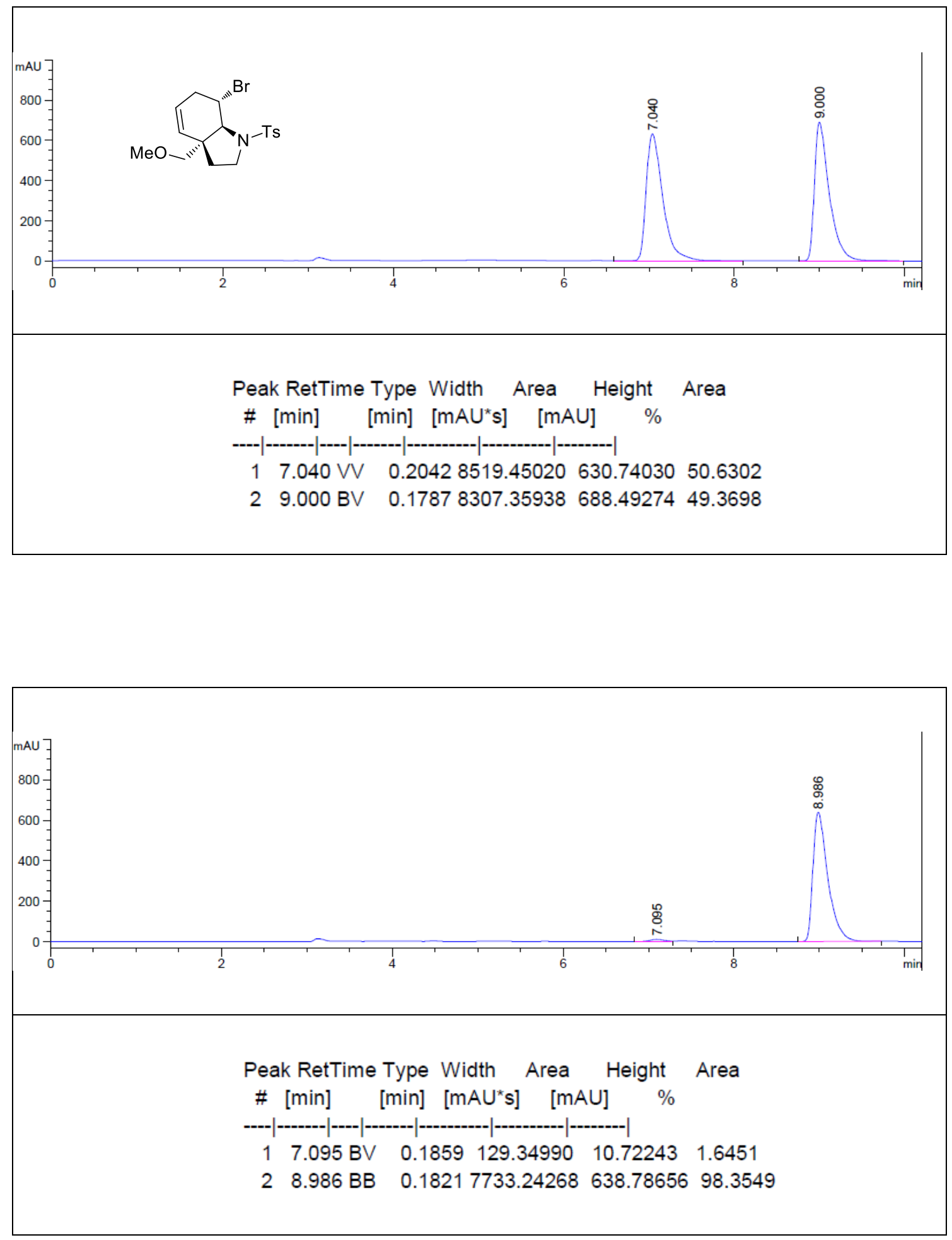
HPLC Analysis of 2p
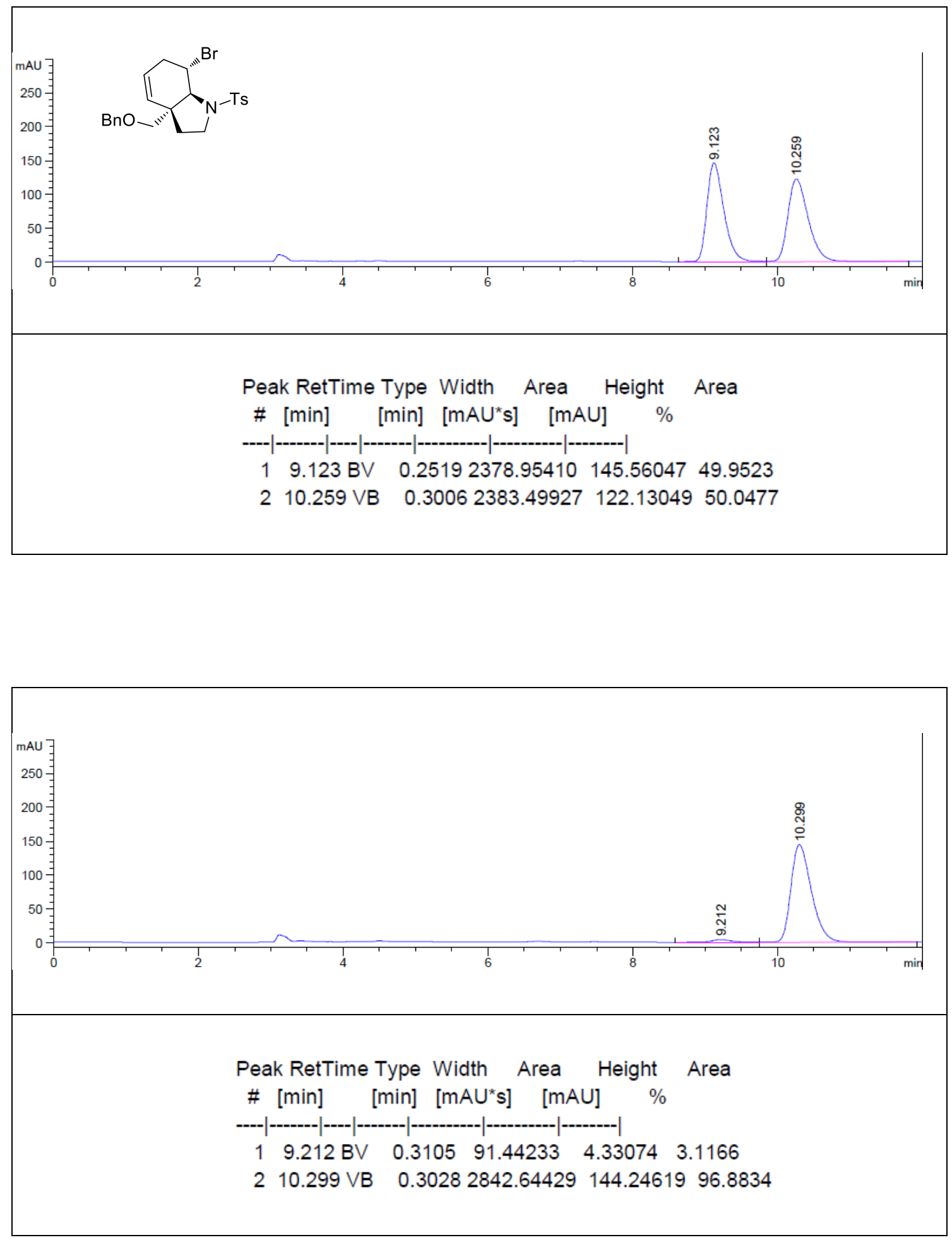
HPLC Analysis of 2q
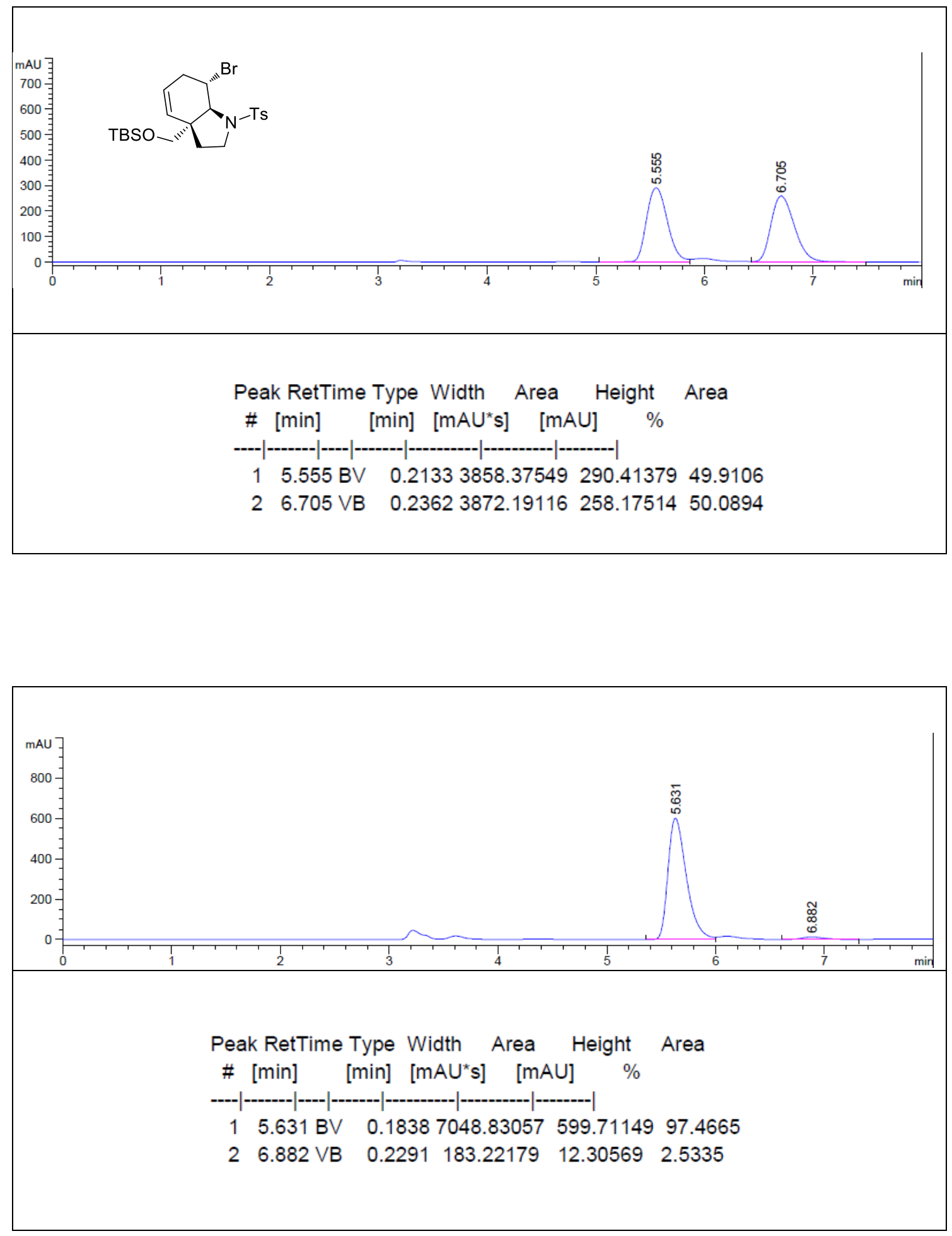
HPLC Analysis of $2 r$
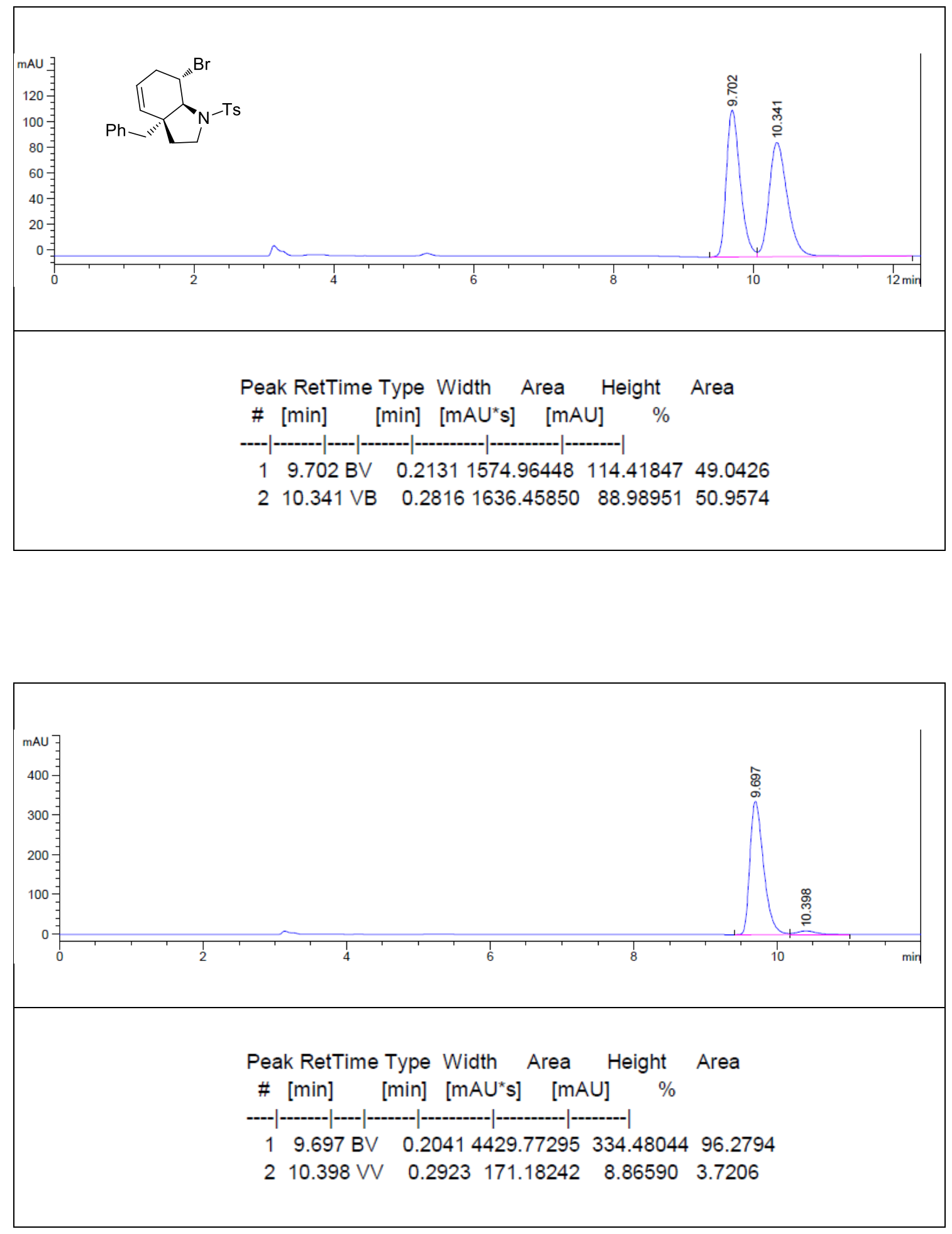
HPLC Analysis of $2 \mathrm{~s}$
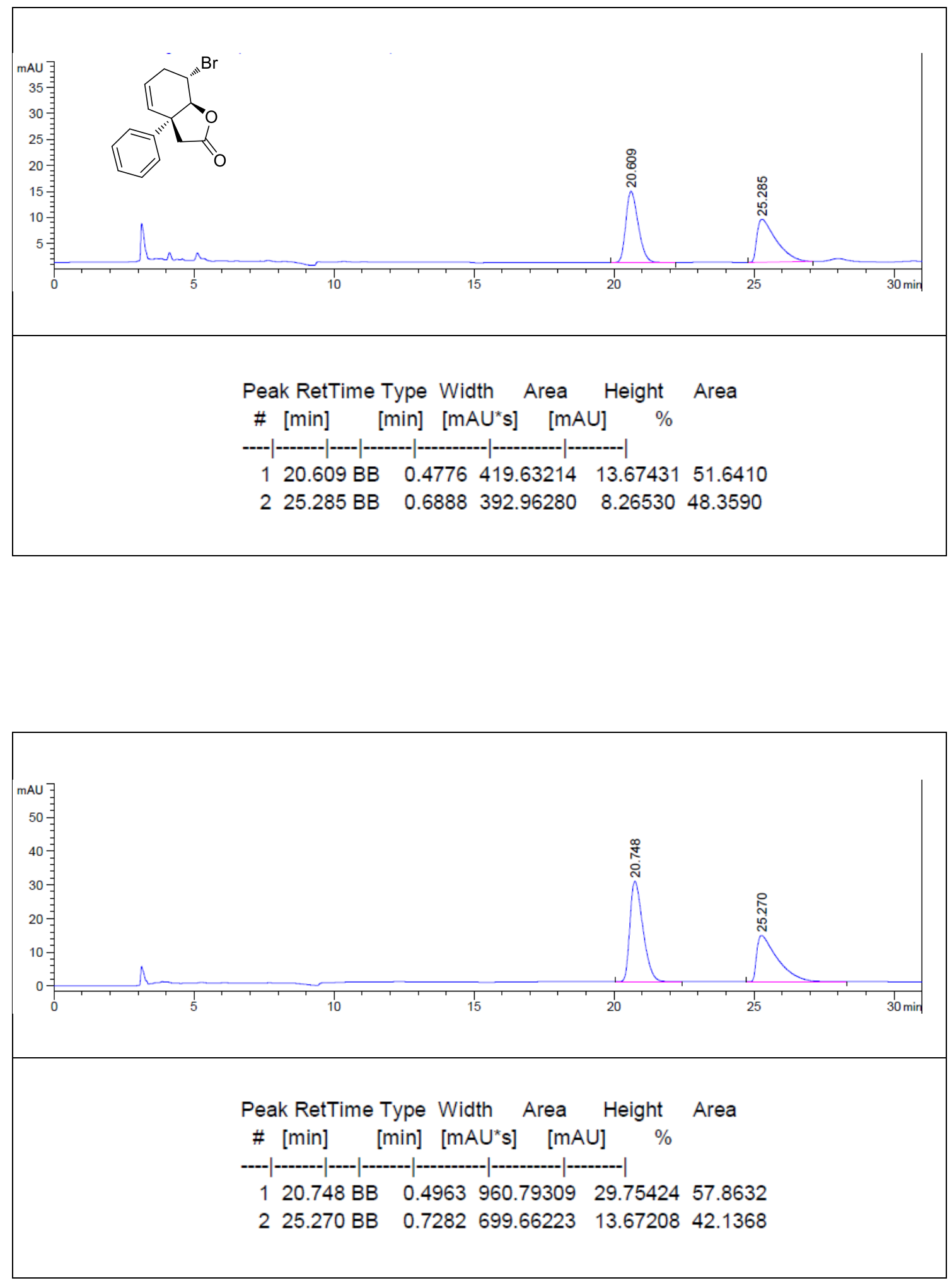


\section{Crystallographic Data of Compounds 2a, 2m and 2a'}

The single crystals (CCDC Number: 2103020, 2103021 and 2112349) of compounds 2a, 2m and 2a' were obtained by slow evaporation of the solution of hexanes / dichloromethane. A suitable single crystal for X-ray diffraction was mounted on an Agilent SuperNova-CCD XRay diffractometer, Eos fitted with Mo K $\alpha$ radiation $(\lambda=0.71073 \AA)$ at $295 \mathrm{~K}$. Data collection and unit cell refinement were executed by using CrysAlisPro software. Empirical absorption correction using spherical harmonics, implemented in SCALE3 ABSPACK scaling algorithm. The structure was solved with the XT structure solution program using Intrinsic Phasing and refined with the XL refinement package using Least Squares minimisation in Olex2. All nonhydrogen atoms were refined anisotropically. Hydrogen atoms were placed in ideal positions and refined as riding atoms. The absolute configuration was established by anomalous dispersion effects in diffraction measurements on the crystal. The thermal ellipsoids are shown at $50 \%$ probability level. See below for other details.

\subsection{Crystallographic data of 2a:}

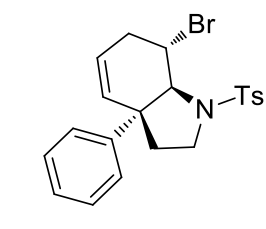

$2 a$

CCDC: 2103020

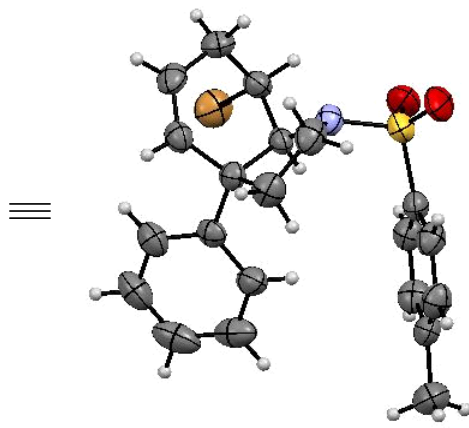

Thermal ellipsoids are scaled to the $50 \%$ probability level.

Table S4. Crystal data and structure refinement for $\mathbf{2 a}$

$\begin{array}{ll}\text { Identification code } & \mathrm{C}_{21} \mathrm{H}_{22} \mathrm{BrNO}_{2} \mathrm{~S} \\ \text { Empirical formula } & \mathrm{C}_{21} \mathrm{H}_{22} \mathrm{BrNO}_{2} \mathrm{~S} \\ \text { Formula weight } & 432.36 \\ \text { Temperature/K } & 293(2) \\ \text { Crystal system } & \text { orthorhombic } \\ \text { Space group } & \mathrm{P} 2{ }_{1} 2{ }_{1} 21 \\ \text { a/A } & 8.1229(7) \\ \text { b/A } & 10.7696(6) \\ \text { c/ } & 22.0965(15)\end{array}$




$\begin{array}{ll}\alpha /^{\circ} & 90 \\ \beta /{ }^{\circ} & 90 \\ \gamma /{ }^{\circ} & 90 \\ \text { Volume/ } \AA^{3} & 1933.0(2) \\ \mathrm{Z} & 4 \\ \rho_{\text {calcg/cm }}{ }^{3} & 1.486 \\ \mu / \mathrm{mm}^{-1} & 2.250 \\ \mathrm{~F}(000) & 888.0 \\ \text { Crystal size/mm } & \\ \text { Radiation } & 0.37 \times 0.36 \times 0.34 \\ 2 \Theta \text { range for data collection/ } & \\ \text { Index ranges } & \mathrm{MoK} \alpha(\lambda=0.71073) \\ \text { Reflections collected } & -9 \leq \mathrm{h} \leq 11,-13 \leq \mathrm{k} \leq 13,-24 \leq 1 \leq 29 \\ \text { Independent reflections } & 7783 \\ \text { Data/restraints/parameters } & 4357\left[\mathrm{R}_{\text {int }}=0.0316, \mathrm{R}_{\text {sigma }}=0.0711\right] \\ \text { Goodness-of-fit on } \mathrm{F}^{2} & 4357 / 0 / 236 \\ \text { Final R indexes }[\mathrm{I}>=2 \sigma(\mathrm{I})] & 1.001 \\ \text { Final R indexes [all data] } & \mathrm{R}_{1}=0.0466, \mathrm{wR}_{2}=0.0662 \\ \text { Largest diff. peak/hole / e } \AA^{-3} & \mathrm{R}_{1}=0.0882, \mathrm{wR}_{2}=0.0775 \\ \text { Flack parameter } & 0.41 /-0.40 \\ & -0.014(7) \\ & \end{array}$

9.2 Crystallographic data of $\mathbf{2} \mathbf{m}$ :
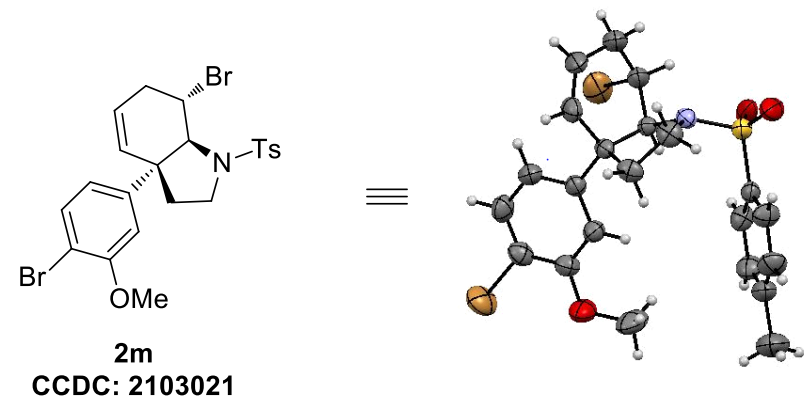

Thermal ellipsoids are scaled to the $50 \%$ probability level. 
Table S5. Crystal data and structure refinement for $\mathbf{2 m}$

\begin{tabular}{|c|c|}
\hline Identification code & $\mathrm{C}_{22} \mathrm{H}_{23} \mathrm{Br}_{2} \mathrm{NO}_{3} \mathrm{~S}$ \\
\hline Empirical formula & $\mathrm{C}_{22} \mathrm{H}_{23} \mathrm{Br}_{2} \mathrm{NO}_{3} \mathrm{~S}$ \\
\hline Formula weight & 541.29 \\
\hline Temperature/K & 293(2) \\
\hline Crystal system & orthorhombic \\
\hline Space group & $\mathrm{P} 2{ }_{1}{ }_{1} 2_{1}$ \\
\hline $\mathrm{a} / \AA$ & $8.1756(2)$ \\
\hline $\mathrm{b} / \AA$ & $8.7210(5)$ \\
\hline$c / \AA$ & $31.6359(13)$ \\
\hline$\alpha /^{\circ}$ & 90 \\
\hline$\beta /{ }^{\circ}$ & 90 \\
\hline$\gamma /{ }^{\circ}$ & 90 \\
\hline Volume $/ \AA^{3}$ & $2255.62(17)$ \\
\hline $\mathrm{Z}$ & 4 \\
\hline$\rho_{\text {calc }} \mathrm{g} / \mathrm{cm}^{3}$ & 1.594 \\
\hline$\mu / \mathrm{mm}^{-1}$ & 3.709 \\
\hline $\mathrm{F}(000)$ & 1088.0 \\
\hline Crystal size $/ \mathrm{mm}^{3}$ & $0.35 \times 0.28 \times 0.26$ \\
\hline Radiation & $\operatorname{Mo~K\alpha }(\lambda=0.71073)$ \\
\hline $2 \Theta$ range for data collection $/{ }^{\circ}$ & 6.832 to 58.154 \\
\hline Index ranges & $-10 \leq \mathrm{h} \leq 6,-11 \leq \mathrm{k} \leq 11,-43 \leq 1 \leq 30$ \\
\hline Reflections collected & 9487 \\
\hline Independent reflections & $5094\left[\mathrm{R}_{\text {int }}=0.0298, \mathrm{R}_{\text {sigma }}=0.0608\right]$ \\
\hline Data/restraints/parameters & $5094 / 0 / 264$ \\
\hline Goodness-of-fit on $\mathrm{F}^{2}$ & 1.057 \\
\hline Final $R$ indexes $[\mathrm{I}>=2 \sigma(\mathrm{I})]$ & $\mathrm{R}_{1}=0.0485, \mathrm{wR}_{2}=0.0786$ \\
\hline Final $\mathrm{R}$ indexes [all data] & $\mathrm{R}_{1}=0.0862, \mathrm{wR}_{2}=0.0950$ \\
\hline
\end{tabular}


Largest diff. peak/hole / e $\AA^{-3} \quad 0.37 /-0.54$

Flack parameter

$-0.011(6)$

9.3 Crystallographic data of $\mathbf{2} \mathbf{a}^{\prime}$ :
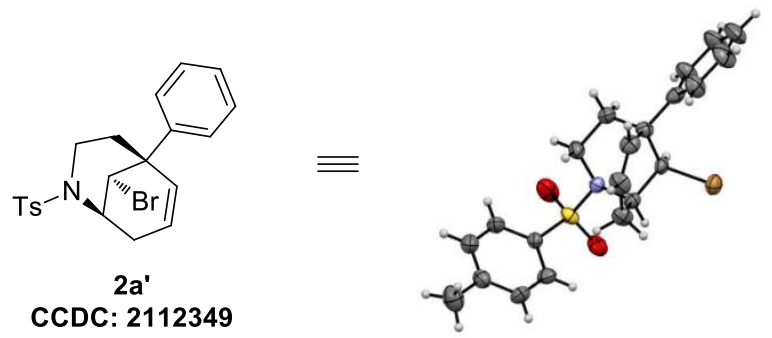

Thermal ellipsoids are scaled to the $50 \%$ probability level.

Table S6. Crystal data and structure refinement for $\mathbf{2 a}$ '

Identification code

Empirical formula

Formula weight

Temperature/K

Crystal system

Space group

$\mathrm{a} / \AA$

$\mathrm{b} / \AA$

$\mathrm{c} / \AA$

$\alpha /^{\circ}$

$\beta /{ }^{\circ}$

$\gamma /{ }^{\circ}$

Volume $/ \AA^{3}$

Z

pcalcg/ $\mathrm{cm}^{3}$

$\mu / \mathrm{mm}^{-1}$
$\mathrm{C}_{21} \mathrm{H}_{22} \mathrm{BrNO}_{2} \mathrm{~S}$

$\mathrm{C}_{21} \mathrm{H}_{22} \mathrm{BrNO}_{2} \mathrm{~S}$

432.36

293(2)

monoclinic

$\mathrm{P} 2_{1}$

9.9914(7)

$8.2749(5)$

$11.8336(7)$

90

94.710(6)

90

975.07(11)

2

1.473

2.230 
$\mathrm{F}(000)$

Crystal size $/ \mathrm{mm}^{3}$

Radiation

$2 \Theta$ range for data collection/ ${ }^{\circ}$

Index ranges

Reflections collected

Independent reflections

Data/restraints/parameters

Goodness-of-fit on $\mathrm{F}^{2}$

Final $\mathrm{R}$ indexes $[\mathrm{I}>=2 \sigma(\mathrm{I})]$

Final $\mathrm{R}$ indexes [all data]

Largest diff. peak/hole / e $\AA^{-3}$

Flack parameter
444.0

$0.33 \times 0.31 \times 0.29$

$\operatorname{Mo} \operatorname{K} \alpha(\lambda=0.71073)$

6.91 to 58.104

$-12 \leq \mathrm{h} \leq 12,-10 \leq \mathrm{k} \leq 11,-15 \leq 1 \leq 15$

7466

$4375\left[R_{\text {int }}=0.0425, R_{\text {sigma }}=0.0813\right]$

$4375 / 1 / 236$

1.097

$\mathrm{R}_{1}=0.0604, \mathrm{wR}_{2}=0.1388$

$\mathrm{R}_{1}=0.0813, \mathrm{wR}_{2}=0.1599$

$1.06 /-0.47$

$-0.003(12)$ 